\title{
Extraction, bioactivity, and stability of wheat bran
}

\author{
alkylresorcinols
}

by

Aynur Gunenc

A thesis submitted to the Faculty of Graduate and Postdoctoral Affairs in partial fulfillment of the requirements for the degree of

Doctor of Philosophy

in

Chemistry

(Food Science and Nutrition)

Carleton University

Ottawa, Ontario

(C) 2014, Aynur Gunenc 


\section{Abstract}

Whole grain intake may be linked to a lowered risk of chronic diseases and such positive effects might be attributed to phenolic lipids, alkylresorcinols (ARs), found in cereal bran. This study aimed to characterize ARs in wheat bran (WB), investigate the influence of environmental factors on AR composition, measure AR bioactivity including antioxidant activity (AA) and investigate AR stability during baking. Moreover, the prebiotic potential of WB-soluble dietary fibre (SDF) was explored using yogurt models. Specific objectives were achieved by the following projects.

The effects of cultivar, and region on the ARs content in 24 wheat cultivars grown in Ontario were studied, by GC-MS and their AA were evaluated. TPC (3.0 to $58.0 \mathrm{mg}$ FAE/g), DPPH (5 to 68\%), ORAC (6.0 to $94.0 \mu \mathrm{mol} \mathrm{TE} / \mathrm{g}$ ) and ARs (21.0 to 1522.0 $\mu \mathrm{g} / \mathrm{g})$ of WB extracts were significantly affected by location and cultivar $(P<0.05)$.

The stability of WB-ARs was studied using different bread formulations. Bread ARs (1.1 to $82.9 \mathrm{mg} / 100 \mathrm{~g}$ ) were heat stable during baking at $255^{\circ} \mathrm{C}$ for $12 \mathrm{~min}$. A positive correlation was observed between TPC and ORAC $\left(R^{2}=0.90\right)$.

Two extraction methods were used to compare the AR contents of WB. The $\%$ ARs per extract using acetone versus $\mathrm{SC}-\mathrm{CO}_{2}$ extraction of both WB samples studied were in the range of $10.9 \%-15.6 \%$ and $5.1 \%-6.6 \%$, respectively.

The prebiotic potential of WB-SDF to enhance lactic acid bacteria (LAB) survival in yogurt models was explored. Total DF (53\%) was counted as a sum of SDF (6\%) and IDF (47\%). HPLC analysis of DF fractions treated with alkaline hydrolysis showed that IDF (84.2\%) had a higher phenolic acid (PA) content than SDF (15.8\%). There was a significant difference in total bacterial count $(9.1 \log \mathrm{CFU} / \mathrm{mL}), \mathrm{pH}(4.8)$ and TTA 
(1.4\%) in yogurt samples containing 4\% WB compared to controls during the four week cold storage period $\left(4^{\circ} \mathrm{C}\right)$.

These results could lead to practical applications of WB-ARs and SDF in the functional foods and nutraceutical industry. Furthermore, increased WB utilization will reduce Agri-Food leftovers, thus improving environmental sustainability. 


\section{Acknowledgements}

Firstly, I would like to express my deepest gratitude to my academic supervisor, Dr. Farah Hosseinian for giving me the opportunity to carry out the research project presented in this thesis. I was fortunate to have met her right after my M.Sc. study and honored to be her first graduate student in her academic career in 2009. From the day I met her, she has always been encouraging, enthusiastic, and hardworking about her research. Her passion for science, teaching and research led me joining her research and pursuing a $\mathrm{Ph} \mathrm{D}$. She has provided the scientific and moral support, and has guided me throughout my graduate student life.

The experimental part of this study was carried out mainly in the Food Science and Nutrition Laboratory of the Chemistry Department at Carleton University, with some parts being done in the University of Ottawa Chemistry Department, as well as in the Culinary Management Program of the School of Hospitality and Tourism at Algonquin College. Several people have contributed time and expertise to this thesis and helped me during the years.

I am very grateful for the guidance of the many faculty and staff members in our Chemistry Department who have cultivated my interest in science and encourage me along the way including, Dr. Apollinaire Tsopmo, Dr. Veronic Bezaire, Dr. Tyler Avis, Dr. Jeff Smith, and Dr. Jeff Manthrope. Moreover, I thank you Prof. Bob Burk for supporting me and for taking time to give advice in achieving my goals. I would also like to thank Chantelle Gravelle and Marilyn Stock for their kindness and secretarial help, as well as grateful thanks to Jim Logan and Tony O’Neil for their technical support.

I would like to thank every member of Dr. Hosseinian's Research Group, 
especially, Research Associate Dr. Mehri Hadinezhad for giving important criticism and lots of time to this project. I would also like to thank the graduate students Rania Agil, and Shana Cameron for their supports any time I needed it, especially for proof reading some documents. I wish to thank Christina Alswiti, Julia Gliwa, Natasha Ramkay and Majed Alshehri who have been ready to help in any matter. Moreover, I would like to thank the undergraduate students: Mergan Ghiyasvand, Ibrahim Farah, Abdulrahman Hashem, and Farah Alasadi for their support and help in sample preparations.

Thanks are due to Dr. Koushik Seetharaman and Lily Tamburic-Ilincic from Guelph University and Judith Fregeau-Reid from Agriculture Canada for providing the wheat bran samples used at various stages of experimentation in this thesis, and for the encouragement, support, and advice.

All my friends and relatives have supported me in their own ways. I thank Emine Sahin for visiting me from back home and supporting me in my carrier path. I thank my parents, Zeycan and Mustafa Karaduman, for being encouraging and giving support when I needed it. Their versatile support at all times has made my path much lighter.

I am deeply grateful for the financial support provided by the Ontario Ministry of Agriculture, Food and Rural Affairs (OMAFRA), the Canadian Foundation for Innovation (CFI), and Carleton University.

Finally, I dedicate this thesis to my family with special thanks to my dear husband, Metin Gunenc, and my beloved sons, Bilal and Cemal Gunenc. Thank you for your understanding, giving me the strength to hold on to my ideas, encouraging me to pursue my dreams, and for always giving me support when I needed it. I am so proud of my family and wish you all the best. 


\section{List of Publications}

This thesis is based on the following original publications.

1. Gunenc, A., HadiNezhad, M., Tamburic-Ilincic, L., Mayer, P.M., Hosseinian, F. Effects of region and cultivar on alkylresorcinols content and composition in wheat bran and their antioxidant activity, Journal of Cereal Science, 2013, $57: 405-410$

2. Gunenc, A., Tavakoli, H., Seetharaman, K., Mayer, P.M., Fairbanks, D., and Hosseinian, F. Stability and antioxidant capacity of alkylresorcinols in Canadian hard and soft red wheat bran during baking, Food Research International, 2013, $51: 571-578$

3. Gunenc, A., and Hosseinian, F. Comparision of traditional and super critical carbon dioxide extraction of alkylresorcinols and their homolog profile and bioactivity, Manuscript.

4. Gunenc, A., and Hosseinian, F. Wheat bran enhances lactic acid bacterial survival and growth in yogurt and hold high antioxidant capacity. Manuscript. 


\section{Table of Contents}

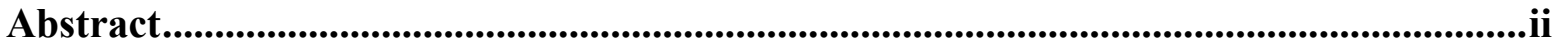

Acknowledgements ...............................................................................................................................iv

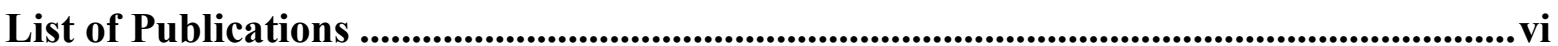

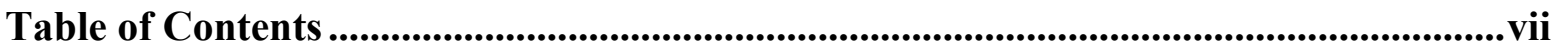

List of Tables ......................................................................................................................................

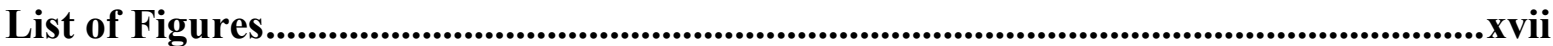

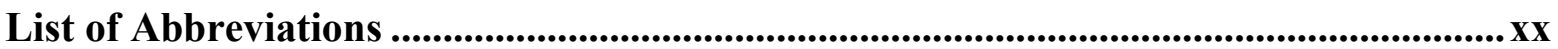

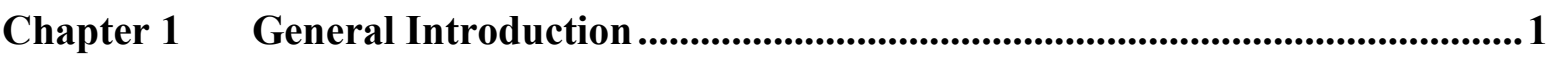

1.1 Significance of wheat .....................................................................................................................

1.2 Wheat structure .....................................................................................................................

1.3 Wheat bran production ............................................................................................................

1.4 Wheat nutrition ............................................................................................................................

1.5 Alkylresorcinols structure and sources.............................................................................

1.6 Alkylresorcinols in cereals ................................................................................................11

1.7 Biosynthesis of alkylresorcinols ........................................................................................13

1.8 Extraction of alkylresorcinols ............................................................................................16

1.9 Supercritical carbon dioxide $\left(\mathrm{SC}-\mathrm{CO}_{2}\right)$ extraction of $\mathrm{ARs}$.......................................16

1.10 ARs analysis methods ............................................................................................................17

1.10.1 Colorimetric determination of ARs ............................................................. 18

1.10.2 Thin layer and paper chromatography ......................................................... 19

1.10.3 High performance liquid chromatography (HPLC) .................................. 19 


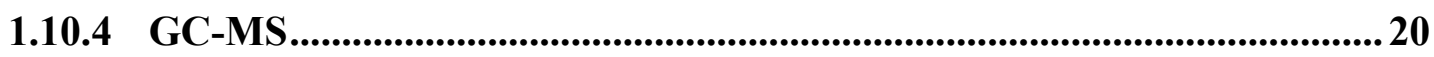

1.11 Bioactivity of alkylresorcinols ..........................................................................................20

1.11.1 Free radicals and antioxidant activity .......................................................21

1.11.2 Oxygen radical absorbance capacity (ORAC)............................................24

1.11.3 Diphenyl-picryhydrazyl (DPPH) ...................................................................25

1.12 Prebiotic activity ......................................................................................................................25

1.13 Thesis objectives .......................................................................................................................29

1.14 Contribution of the authors to the manuscript based chapters ...............................32

1.15 Connecting Statement to Chapter 2....................................................................................34

Chapter 2 Effects of region and cultivar on alkylresorcinols content and composition in wheat bran and their antioxidant activity .......................................................35

2.1 Abstract …….........................................................................................................................35

2.2 Introduction ....................................................................................................................................36

2.2.1 Climatic conditions affect wheat production ...............................................36

2.2.2 Wheat bran-phytochemicals affected by environmental factors .............37

2.2.3 Alkylresorcinols in wheat bran ........................................................................38

2.2.4 Objectives ...................................................................................................39

2.3 Materials and methods.................................................................................................39

2.3.1 Samples.....................................................................................................................39

2.3.2 Materials...................................................................................................... 41

2.3.3 Extraction of alkylresorcinols (ARs) .............................................................42

2.3.4 GC-MS analysis .............................................................................................. 43

2.3.5 Measuring total phenolic content (TPC) of wheat bran extracts............44 
2.3.6 Measuring antioxidant activity by DPPH scavenging activity assay of wheat bran extracts.

2.3.7 Oxygen radical absorbance capacity (ORAC) assay of wheat bran extracts 45

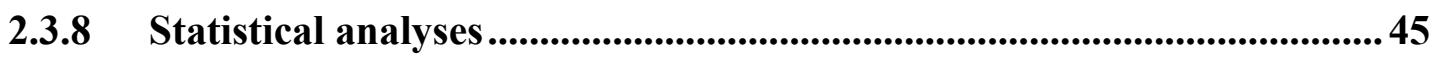

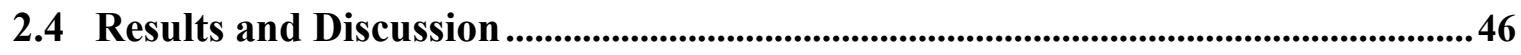

2.4.1 ARs analysis of wheat bran using GC-MS ................................................ 46

2.4.1.1 Total ARs content and related composition ............................... 46

2.4.1.2 Effects of region and cultivar ................................................. 51

2.4.1.3 The relative $\%$ of homologue composition ..................................53

2.4.2 TPC of wheat bran extracts.................................................................5

2.4.3 Antioxidant activity of wheat bran extracts by DPPH scavenging

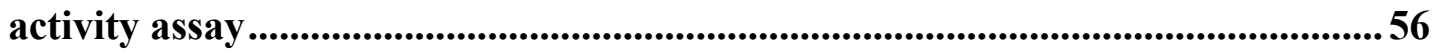

2.4.4 ORAC assay of wheat bran extracts.......................................................58

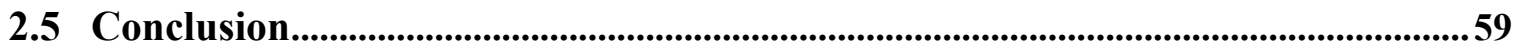

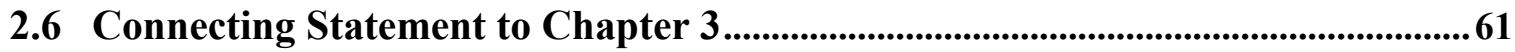

Chapter 3 Stability and antioxidant activity of alkyresorcinols in breads enriched with hard and soft wheat bran ...............................................................62

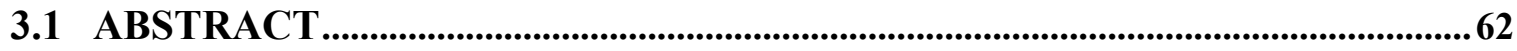

3.2 Introduction..................................................................................................................................6

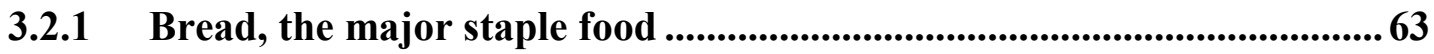

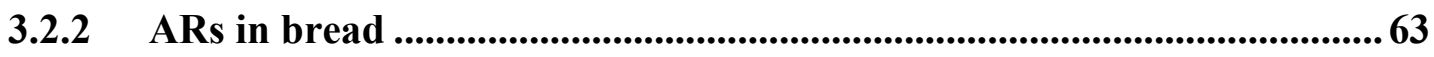

3.2.3 Objectives ................................................................................................ 64 


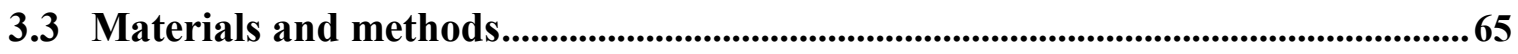

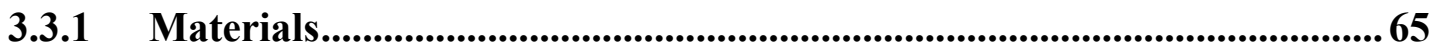

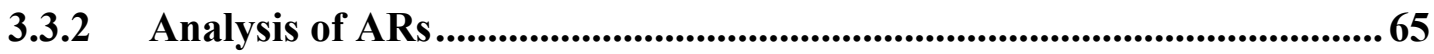

3.3.2.1 Extraction of ARs in wheat bran samples ..................................65

3.3.2.2 Extraction of ARs in bread samples......................................... 66

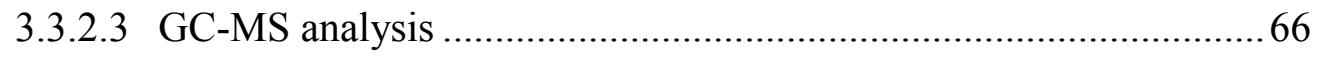

3.3.3 Bread making procedure .................................................................................... 66

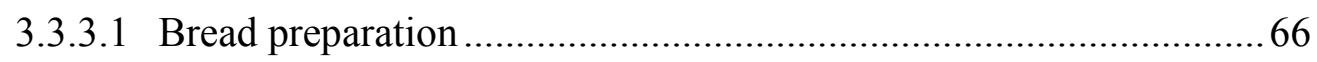

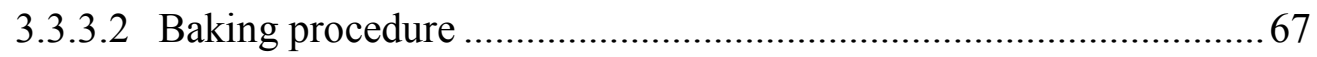

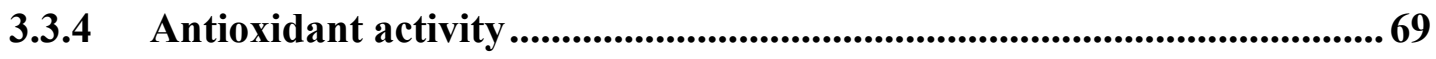

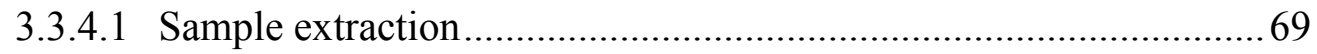

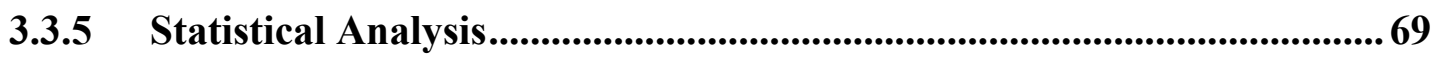

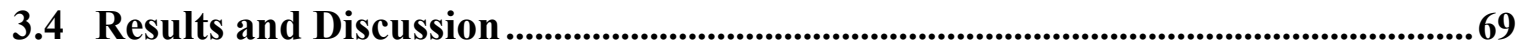

3.4.1 AR content and homolog composition in wheat bran ............................. 69

3.4.2 AR content and homologue composition in bread samples ..................... 77

3.4.3 Effects of ARs on bread physical quality .......................................... 78

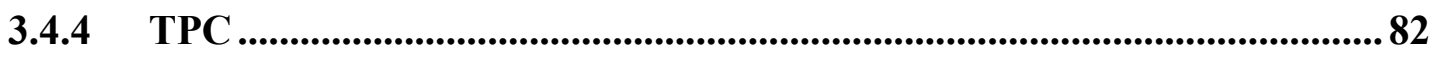

3.4.5 Antioxidant activity by ORAC ...................................................................... 84

3.4.6 Antioxidant activity by \%DPPH.........................................................85 


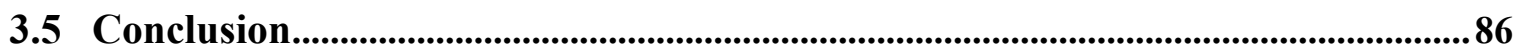

3.6 Connecting Statement to Chapter 4...............................................................................87

Chapter 4 Comparison of traditional and supercritical carbon dioxide extractions of ARs, their homolog profile, and bioactivity ...................................................88

4.1 Abstract ……........................................................................................................................

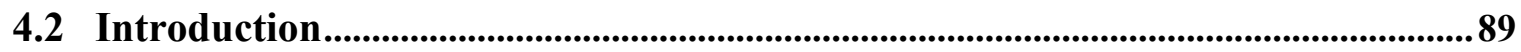

4.3 Materials and methods........................................................................................................91

4.3.1 Materials............................................................................................................91

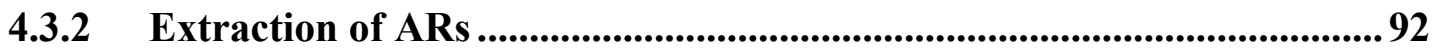

4.3.3 Different solvent extractions for antioxidant activity measurements....94

4.3.4 Fractional extraction (soluble free, soluble conjugated, and bound phenolics) .......................................................................................................................94

4.3.5 HPLC analysis ..................................................................................................99

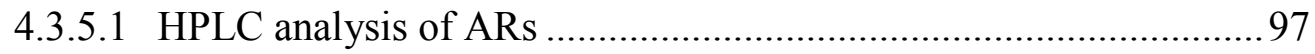

4.3.5.2 HPLC analysis of phenolics ..................................................... 98

4.3.6 Antioxidant activity measurements ............................................................99

4.3.7 Statistical analysis..........................................................................................99

4.4 Results and discussion ......................................................................................................... 100

4.4.1 AR content and homolog composition by traditional method ...............100

4.4.2 AR content and homolog composition by $\mathrm{SC}-\mathrm{CO}_{2}$ method ....................103

4.4.3 Phenolic acids and flavonoids content and composition analysis of fractional extraction of phenolics ...................................................................................107

4.4.4 Antioxidant activity ........................................................................................114 
4.5 Conclusion.

4.6 Connecting Statement to Chapter 5

Chapter 5 Wheat bran enhances lactic acid bacterial survival and growth in yogurt and hold high antioxidant capacity.

5.1 ABSTRACT.

5.2 Introduction

5.3 Materials and method

5.3.1 Materials.

5.3.2 Sample preparation

5.3.3 Probiotic activity.

5.3.3.1 Milk Preparation and Sample Concentration

5.3.3.2 Microbial cultures

5.3.3.3 Microbial count

5.3.3.4 $\mathrm{pH}$ and total titratable acidity (TTA)

5.3.4 Extraction of prebiotics (water extractable polysaccharide, WEP) ....127

5.3.5 Antioxidant activity 128

5.3.6 Total dietary fiber (DF): soluble (SDF) and insoluble (IDF) extraction 128

5.3.7 Phenolic acid extraction from SDF and IDF 128

5.3.8 Statistical analysis 
5.4 Results and discussion ..................................................................................................... 129

5.4.1 Optimizing HRWB concentration .................................................... 129

5.4.2 Prebiotic activity of HRWB addition on microbial count (log

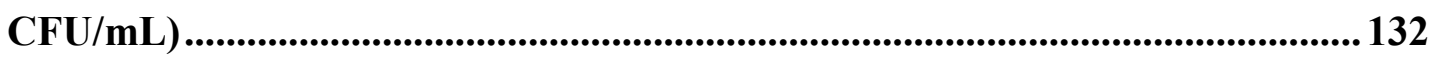

5.4.3 Prebiotic activity of HRWB addition on $\mathrm{pH}$ and TTA ....................... 135

5.4.4 Total dietary fiber determination .................................................. 139

5.4.5 Phenolic acids and flavonoids content and composition analysis of

fractional extraction of phenolics .................................................................... 139

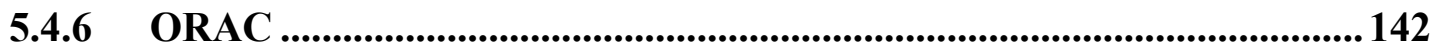

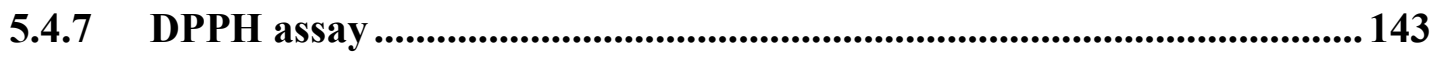

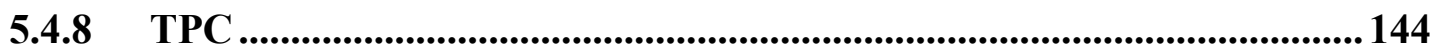

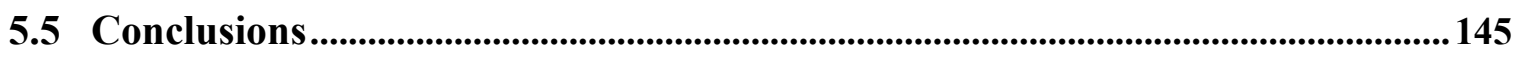

Chapter 6 General Conclusion and Future Directions ............................................146

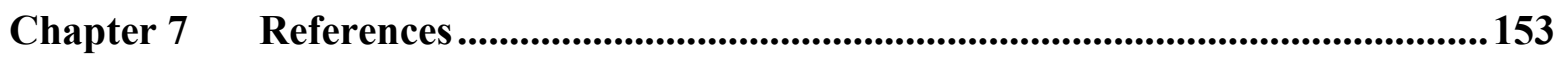




\section{List of Tables}

Table 1-1 Average content $(\mathrm{g} / 100 \mathrm{~g})$ of the major bioactive compounds in whole-grain wheat, wheat bran, and germ fractions $(\%)^{*}$, adapted from Fardet $^{30} \ldots \ldots \ldots \ldots \ldots \ldots \ldots \ldots . . .6$

Table 1-2 Major bioactive components ( $\mu \mathrm{g} / \mathrm{g}$ dry matter) contained in whole grain wheat, rye, oats, and barley, adopted from Frolich et al. ${ }^{31}$............................................. 7

Table 1-3 Various 5- $n$-alkyl, 5-alkenyl-, 5-(oxoalkyl)-, 5-(oxoalkenyl)-, and 5(hydroxylalkenyl)-resorcinols isolated from wheat and rye, adopted from Ross et $\mathrm{al}^{34}$

Table 1-4 Alkylresorcinol content of whole cereal grains, bran and flour ${ }^{35,45,46}$............ 11

Table 2-1 Average 1999-2008 Canadian wheat production and yield by province ${ }^{126}$.... 37

Table 2-2 The average precipitation $(\mathrm{mm})$, and temperatures $\left({ }^{\circ} \mathrm{C}\right)$ for each region........ 40

Table 2-3 Total ARs content in $\mu \mathrm{g} / \mathrm{g}$, and related \% saturated (Sat), unsaturated (Unsat)

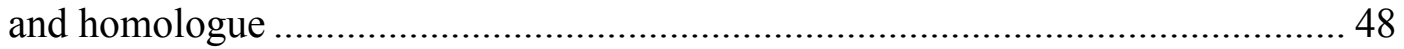

Table 2-4 Alkylresorcinols (ARs) analysis results for cultivar and region effects .......... 52

Table 2-5 TPC (mg FAE /g), \% DPPH, and ORAC ( $\mu \mathrm{mol} \mathrm{TE} / \mathrm{g}$ ) values* of WB extracts 55

Table 2-6 Antioxidant assays of individual and mixture of ARs standard ...................... 59

Table 3-1 All bread trials and recipes for bran samples: HRWB (hard red wheat bran) and SRWB (soft red wheat bran)

Table 3-2 Total AR content (mg/100 g), \% saturated (Sat), unsaturated (Unsat) and homologue (C15-C25) ARs in wheat bran, bran-residue and white flour. 73 
Table 3-3 Total content (mg/100 g), saturated (Sat), unsaturated (Unsat) and homologue composition (\%) of ARs in all bread trials 76

Table 3-4 Effects of ARs on bread physical quality: The inner temperature, height and weight of bread samples after $1 \mathrm{~h}$ cooling........ 79

Table 3-5 ORAC, \% DPPH, and TPC values of wheat bran, bran-residue, white flour and bread samples. 83

Table 4-1 Detailed information about all WB samples used for this study 91

Table 4-2 Comparison of SC-CO2 parameters for ARs extraction of cereal bran 94

Table 4-3 The gradient program for HPLC analysis of ARs 97

Table 4-4 The gradient program for HPLC analysis of phenolic acid and flavonoids ..... 98

Table 4-5 Total ARs and homolog contents (mg/100g WB) of different wheat cultivars extracted by acetone. 101

Table 4-6 Total ARs and homolog contents (mg/100g WB) of HRWB and SRWB samples extracted by $\mathrm{SC}-\mathrm{CO}_{2}$ 106

Table 4-7 Phenolic acid (PA) content (mg/g WB) and composition of HRWB and SRWB fractions including soluble free (A), soluble conjugated (B), and bound (C) ..... 108

Table 4-8 Phenolic acid and flavonoids standards used for HPLC analysis and their retention times $(\mathrm{RT})$ in minute

Table 4-9 Flavonoids contents (FC, mg/g of WB) and composition of HRWB and SRWB fractionals including soluble free (A), soluble conjugated (B), and bound (C) ... 113

Table 4-10 TPC (mg FAE/g WB) values of different wheat cultivars extracted by three different solvent systems 115

Table 4-11 DPPH (\%) of wheat bran extracts with different solvent systems 116 
Table 4-12 ORAC ( $\mu$ mole of TE/g WB) values of different wheat cultivars extracted with different solvent systems. 118

Table 4-13 The two way ANOVA for cultivar and solvent effects on antioxidant activity measurements; TPC, DPPH and ORAC

Table 5-1 The experimental design used to evaluate the effect of addition of HRWB sample on probiotic viability in different yogurt trials. 126

Table 5-2 Phenolic acid (PA) content and composition ( $\mathrm{mg} / \mathrm{g}$ of sample) of SDF, IDF and HRWB 139

Table 5-3 Flavonoids content (FC in $\mathrm{mg} / \mathrm{g}$ of sample) of SDF, IDF and HRWB 142

Table 5-4 ORAC ${ }^{1}, \mathrm{DPPH}^{2}$ and $\mathrm{TPC}^{3}$ values of $\mathrm{WEP}^{4}$ 143 


\section{List of Figures}

Figure 1-1 The three wheat fractions: bran, germ, and endosperm, adopted from Surget,

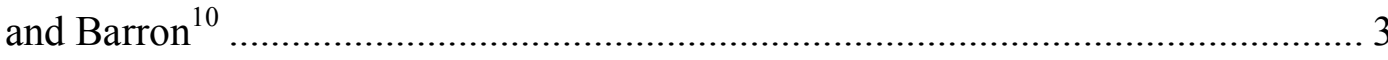

Figure 1-2 The 5-n-alkylresorcinol structure, modified from Ross et al. ${ }^{34}$ ………........... 8

Figure 1-3 Typical percent distribution of the alkylresorcinol homologues C15:0-C25:0 found in rye, ${ }^{46}$ common wheat,${ }^{47}$ durum wheat,${ }^{55,56}$ triticale, ${ }^{46}$ and barley, ${ }^{46,48}$ modified from Söderholm ${ }^{57}$ 13

Figure 1-4 The biosynthesis of alkylresorcinols and various polyketide (triketide, tetraketide and pentaketide) resorcylic acids, adopted from Yu et al. ${ }^{58}$ 15

Figure 1-5 Chemical structures of ferulic acid and diferulic acid identified in plant cell walls, adopted from de Vries et al. ${ }^{119}$ 27

Figure 1-6 The structure of ferulic acid esterified to units of arabinoxylan. A: ferulic acid linked to $0-5$ arabinose chain of arabinoxylan; B: $\beta-1,4-$ linked xylan backbone; $\mathrm{C}$ : $\alpha-1,2$-linked L-arabinose. ${ }^{117}$ 27

Figure 2-1 The map of Ontario regions, adopted from Ontario Ministry of tourism, culture and sport ${ }^{139}$ 40

Figure 2-2 A) GC-MS of derivitized ARs standards (C15-C25) and the fragmentation pattern, B) GC-MS of cultivar Emmit grown in Ridgetown region 50

Figure 3-1 The bread making procedure. 68

Figure 3-2 GC-MS results of ARs from HRWB before baking (A), and in bread 1D (30\%wheat bran plus $2 \%$ crude ARs extract) (B) 72 
Figure 3-3 Breads baked with 100\% white flour (A), 30\% non-extracted wheat bran (1B, 2B), 30\% extracted wheat bran (1C, 2C) and 30\% non-extracted wheat bran and $2 \%$ crude ARs extract added (1D, 2D)

Figure 4-1 SC- $\mathrm{CO}_{2}$ extraction instrument in Dr. Hosseinian's research lab at Carleton University. The letters A, B, C, D, E and F represent the extraction vessel, collector-1, collector-2, automated back pressure regulator (ABPR), co-solvent pump, and $\mathrm{CO}_{2}$ pump, respectively .................................................... 93

Figure 4-2 Soluble free, soluble conjugated and bound phenolic acid extractions procedures (modified from Kim et.al). ${ }^{172}$ 96

Figure 4-3 HPLC chromatograms of ARs standard mixture (A) and HRWB sample (B).The numbers represent each ARs homologue including C15:0 (1), C17:0 (2), $\mathrm{C} 19: 0$ (3), C21:0 (4), C23:0 (5), and C25:0 (6) 100

Figure 4-4 HPLC chromatograms of phenolic acid standard (A), SRWB soluble conjugated phenolic acids (B), flavonoid standards (C) (Look at Table 4.8 for each standard and corresponding retention times)...... 110

Figure 5-1 A) Dialysis of water extractable polysaccharides, B) Resultant yogurt products with various concentrations of hard red wheat bran (HRWB), C) Total microbial count $(\log \mathrm{CFU} / \mathrm{mL})$ of lactic acid bacteria in yogurt containing starter cultures of Lactobacillus bulgaricus and Streptococcus thermophiles with different concentrations of HRWB including $0,2,4,6$, and $8 \%$ over 28 days cold storage at $4{ }^{\circ} \mathrm{C}$. 131

Figure 5-2 Total microbial count $(\log \mathrm{CFU} / \mathrm{mL})$ in control yogurt $(\mathrm{Y}, \mathrm{Y}+1, \mathrm{Y}+2$, $\mathrm{Y}+1+2)$, and yogurt with $4 \%$ wheat bran $(\mathrm{YB}, \mathrm{YB}+1, \mathrm{YB}+2, \mathrm{YB}+1+2)$. Number 1 , 
and 2 represent probiotic1 and 2. Different letters in columns in the same day are significantly different $(P<0.05)$ in Duncan's multiple range tests 134

Figure 5-3 The $\mathrm{pH}$ values in control yogurt $(\mathrm{Y}, \mathrm{Y}+1, \mathrm{Y}+2, \mathrm{Y}+1+2)$ and yogurt with $4 \%$ wheat bran $(\mathrm{YB}, \mathrm{YB}+1, \mathrm{YB}+2, \mathrm{YB}+1+2)$. Number 1 and 2 represent probiotic1 and 2. Different letters in columns in the same day are significantly different $(P<$ 0.05) in Duncan's multiple range tests. 136

Figure 5-4 \% Total titratable acidity (TTA) values for control yogurts $(\mathrm{Y}, \mathrm{Y}+1, \mathrm{Y}+2$, $\mathrm{Y}+1+2)$ and yogurt with $4 \%$ wheat bran $(\mathrm{YB}, \mathrm{YB}+1, \mathrm{YB}+2, \mathrm{YB}+1+2)$. Number 1 and 2 represent probiotic1 and 2. Different letters in columns in the same day are significantly different $(P<0.05)$ in Duncan's multiple range tests. 138

Figure 5-5 HPLC chromatograms of phenolic acid profile of soluble dietary fiber (A), and insoluble dietary fiber (B) of HRWB. Ferulic acid is the most abundant phenolic acid in both fractions. (Table 4.8 descries each standard and corresponding retention times) 140 


\section{List of Abbreviations}

\begin{tabular}{|c|c|}
\hline AAPH & 2, 2'-azobis (2-amidino-propane) dihydrochloride \\
\hline AA & Antioxidant activity \\
\hline $\mathrm{AH}$ & Antioxidants \\
\hline ARs & Alkylresorcinols \\
\hline AXOS & Arabionoxylan oligosaccharides \\
\hline CFI & Canadian Foundation for Innovation \\
\hline $\mathrm{CFU}$ & Colony forming unit \\
\hline DPPH & 2,2-diphenyl-1-picrylhydrazyl \\
\hline DF & Dietary fiber \\
\hline $\mathrm{E}$ & Environment \\
\hline $\mathrm{F}$ & Fluorescein \\
\hline $\mathrm{FE}$ & Ferulic acid equivalent \\
\hline FOS & Fructooligossacharides \\
\hline $\mathrm{G}$ & Genotype \\
\hline GC-MS & Gas chromatography/mass spectrometry \\
\hline HPLC & High performance liquid chromatography \\
\hline HRWB & Hard red wheat bran \\
\hline IDF & Insoluble DF \\
\hline LDL & Low density lipoproteins \\
\hline $\mathrm{m} / \mathrm{m}$ & mass/mass \\
\hline MRS & Man Rogosa Sharpe \\
\hline
\end{tabular}


OMAFRA

$\mathrm{ON}$

ORAC

ORAS

PDA

PP

RNO

ROS

RT

$\mathrm{SC}-\mathrm{CO}_{2}$

SDF

SRWB

TE

TLC

TPC

TTA

WB

WEP
Ontario Ministry of Agriculture, Food and Rural Affairs

Ontario

Oxygen radical absorbance capacity

2'-oxoalkylresorcyclic acid synthase

Photodiode array detector

Polyphenols

Reactive nitrogen species

Reactive oxygen species

Retention time

Supercritical carbon dioxide

Soluble DF

Soft red wheat bran

Trolox equivalent

Thin layer chromatography

Total phenolic content

Total titratable acidity

Wheat bran

Water extractable polysaccharide 


\section{Chapter 1 General Introduction}

\subsection{Significance of wheat}

Wheat, Tricticum aestivum, is one of the oldest and most widely grown grain crops, followed by corn (maize), and rice. It was first cultivated as a food crop about 10,000 BCE somewhere along the Fertile Crescent in the Near East. ${ }^{1,2}$ Wheat agriculture was considered to be in a small region of southeast Turkey and northeast Syria around the Middle Euphrates (the longest river of Eastern Asia, average coordinates $37^{\circ} 00^{\prime} \mathrm{N}, 38^{\circ} 60^{\prime}$ E). ${ }^{1,3}$ The genus name for wheat, Triticum, comes from the Latin word tero (I thresh), within the grass family Poaceae. The current binomial name, Tricticum aestivum, refers to bread wheat, distinguishing it from tetraloid macaroni wheat (Triticum durum). Most of the grown wheat in the world is the aestivum species. ${ }^{4}$

In Canada, wheat was first cultivated around 1605 near Annapolis Royal, Nova Scotia. ${ }^{5}$ It is believed that Selkirk settlers from Scotland were the first to successfully harvest wheat in Western Canada in the early 1800's. ${ }^{6}$ Currently wheat is grown all over Canada, mainly in the Canadian Prairies with Alberta, Manitoba, and Saskatchewan being the three major growing provinces. ${ }^{7}$ In Canada for the last three years (2010-2012), wheat has been cultivated on 8.2-9.3 million hectares producing about 23.1-26.2 million metric tons. ${ }^{8}$ Canada is one of the largest wheat producers and exporters in the world, with almost $70 \%$ of the produced wheat being exported. Two thirds of the world's wheat production is used for human consumption and about one-sixth is used for livestock feed. The remaining portion is used to produce starch, paste, malt, dextrose, gluten, alcohol and other products. ${ }^{5}$

Wheat exists in more than 30,000 varieties, and is grouped into six major classes 
according to the planting and harvesting dates, as well as the hardness, colour, and shape of the kernels. These classes are as follows: a) hard red spring, b) hard red winter, c) soft red winter, d) hard white wheat, e) soft white wheat, and f) durum. ${ }^{4}$ Hard wheat contains high levels of protein and gluten and is generally used for leavened bread. Common white wheat and soft red winter wheat have low protein content and are used for pastries, cookies, cakes. All-purpose flour is made by combining both hard and soft wheat. Durum wheat is used for pasta, semolina, couscous and Arabic flat breads. These above mentioned properties are influenced by a combination of genetic and environmental factors (genotype $(\mathrm{G})$ and environment (E) and the interaction GxE). For instance, grain hardness is largely determined by the genotype (variety), but the more significant contributors to aspects of quality, such as protein content, are the result of growth conditions such as soil fertility, rainfall, and temperature throughout the growing season, at harvest time, and during storage and transport. ${ }^{4,5}$

\subsection{Wheat structure}

Wheat kernels contain three layers; the endosperm, bran, and germ (Figure 1.1). The endosperm makes up approximately $83 \%$ of the kernel weight and contains $70-75 \%$ of the protein present in the wheat. Also, it is the center of the kernel, rich in cellulose, starch, and gluten, and is surrounded by a single cell aleurone layer. ${ }^{9}$ 


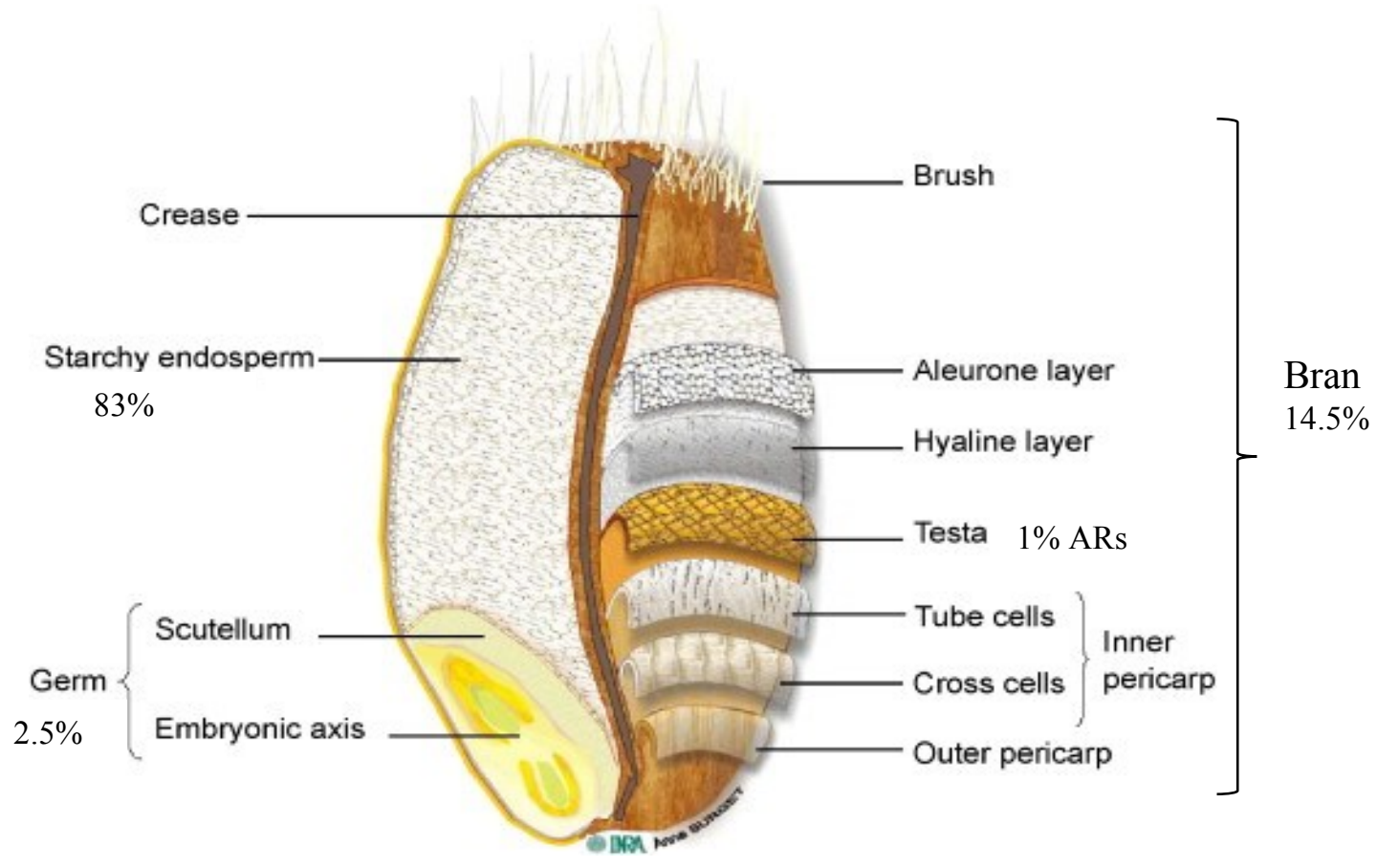

Figure 1-1 The three wheat fractions: bran, germ, and endosperm, adopted from Surget, and Barron $^{10}$

The bran layers enclose the endosperm and are abundant in vitamins, minerals protein, and large amounts of insoluble dietary fiber. ${ }^{9}$ The bran layer is about $14.5 \%$ of the kernel weight. The germ, the embryo of the kernel, accounts for about $2.5 \%$ of the wheat kernel. This layer contains vitamins $\mathrm{B}$, and $\mathrm{E}$, protein $(8 \%)$, fat, and minerals. ${ }^{11,12}$

In the late $1800 \mathrm{~s}$, the pericarp, testa, aleurone, and nucellus (hyaline) bran layers/fractions were described in one of the first research report papers that was published on wheat bran. ${ }^{13}$ Bran layers are generally regarded as the epidermis, hypodermis, cross cells, tube cells, testa, hyaline epidermis, and aleurone. Bran is also referred to by region of the grain such as outer, immediate, or inner layers. ${ }^{14}$ 


\subsection{Wheat bran production}

Wheat bran is a by-product of conventional flour milling and has little value compared with wheat. ${ }^{15,16}$ Generally wheat bran is produced by a roller milling process of the wheat kernels. ${ }^{15}$ This method offers a relatively clean separation of the bran and germ from the endosperm. Moreover, by using this method, the bran fractions are removed with almost all of the bran layers intact. ${ }^{17,18}$

Commercial wheat bran consists of the pericarp, seed coats, and aleurone layers, along with bits and pieces of the endosperm. Wheat bran from conventional milling consists of the outer portions of the kernel with approximately $10-20 \%$ of the endosperm still present. ${ }^{19}$ More detailed information about the modern flour milling process and milling conditions ${ }^{16}$ is available. ${ }^{15}$ In brief, water is added for cleaning, tempering the wheat and toughening the bran. After this process, the endosperm is separated from the bran by milling. Wheat bran yield is about $21.1-36.5 \%$ of the kernel weight. ${ }^{20}$ Generally millers try to produce large $(>2000 \mu \mathrm{m})$ bran pieces and shorts in order to optimize the flour yield. ${ }^{15}$ However, bran thickness is not the only variable determining the postmilling particle size. ${ }^{18}$ Grinding speed, equipment, and wear during milling also influence the geometry of the bran pieces. ${ }^{21}$ The milling efficiency can affect the bran particle size as well as the biological differences in the thickness of the aleurone layer which can determine the amount of clean endosperm that is removed from the bran. ${ }^{22}$

Total global wheat crop production is approximately 600-700 million tonnes of wheat bran annually $\left(\sim 1.4 \times 10^{12} \mathrm{lb} / \sim 2.4 \times 10^{10}\right.$ bushels $) .{ }^{23}$ Global wheat bran consumption increases annually by about $5 \%{ }^{24}$ Wheat bran is commonly used today for many things, such as food, livestock feed, and in cosmetic and biomass products. ${ }^{25}$ 


\subsection{Wheat nutrition}

Wheat is an excellent source of carbohydrates (59.6\%), proteins (11.7\%), lipids (2.2\%), minerals $(1.5 \%)$ and fibre $(13.3 \%) .{ }^{26}$ During the milling process, the removed layers of wheat bran $(14.5 \%)$ and germ $(2.5 \%)$ represent $75 \%$ of the phytonutrients in the wheat kernel (Figure 1.1). ${ }^{27,28}$

The term 'phytochemical' refers to every naturally occurring chemical substance present in plants, especially those that are biologically active. ${ }^{29}$ The known phytochemicals in whole grains and wheat include dietary fiber, vitamins, minerals, lignans, phytoestrogens, phenolic compounds, phytic acid, benzoic, cinnamic acids, anthocyanidins, quinones, flavonols, chalcones, flavones, flavonones, amino phenolic compounds, flavonoids, coumarin derivatives, polyphenols, phytosterines, saponins, catechins, tocotrienls and tocopherol, tannin, caretenoids, ferulic acid and diferulates. ${ }^{27,29}$ The major bioactive compounds and their composition and content in whole wheat grain, wheat bran, and germ fractions are shown in the Table 1.1. 
Table 1-1 Average content $(\mathrm{g} / 100 \mathrm{~g})$ of the major bioactive compounds in whole-grain wheat, wheat bran, and germ fractions $(\%)^{*}$, adapted from Fardet ${ }^{30}$

\begin{tabular}{|c|c|c|c|}
\hline Bioactive compounds & whole-grain wheat ${ }^{\mathrm{a}}$ & Wheat bran ${ }^{\mathrm{a}}$ & Wheat germ ${ }^{\mathrm{a}}$ \\
\hline$\alpha$-Linolenic acid & $\mathrm{NA}^{\mathbf{b}}$ & 0.16 & 0.53 \\
\hline Sulfur compounds & 0.5 & 0.7 & 1.2 \\
\hline Total free glutathione & 0.007 & 0.038 & 0.27 \\
\hline Dietary fiber ${ }^{\mathrm{d}}$ & 13.2 & 44.6 & 17.7 \\
\hline Lignins & 1.9 & 5.6 & 1.5 \\
\hline Oligosaccharides $^{\mathrm{e}}$ & 1.9 & 3.7 & 10.1 \\
\hline Phytic acid & 0.9 & 4.2 & 1.8 \\
\hline Minerals and trace elements & 1.12 & 3.39 & 2.51 \\
\hline Vitamins & 0.0138 & 0.0398 & 0.0394 \\
\hline B-vitamins & 0.0091 & 0.0303 & 0.0123 \\
\hline Vitamin E & 0.0047 & 0.0095 & 0.0271 \\
\hline Carotenoids & 0.00034 & 0.00072 & NA \\
\hline Polyphenols & 0.15 & 1.1 & $>0.37$ \\
\hline Phenolic acids & 0.11 & 1.07 & $>0.07$ \\
\hline Flavonoids & 0.037 & 0.028 & 0.3 \\
\hline Lignans & 0.0004 & 0.005 & 0.0005 \\
\hline Alkylresorcinols & 0.07 & 0.27 & NA \\
\hline Betaine & 0.16 & 0.87 & 0.85 \\
\hline Total choline & 0.12 & 0.17 & 0.24 \\
\hline Total free inositols & 0.022 & 0.025 & $>0.011$ \\
\hline Phytosterols & 0.08 & 0.16 & 0.43 \\
\hline $\begin{array}{l}\text { Policosanol }+ \text { melatonin }+ \text { para }- \\
\text { aminopbenzoic acid }\end{array}$ & 0.00341 & 0.0029 & $>0.00186$ \\
\hline Total & $>15.4$ & 51.5 & $>23.9$ \\
\hline Subtotal (without dietary fibre) & 2.2 & 6.9 & $>6.2$ \\
\hline
\end{tabular}

*Mean $\%$ of bioactive compounds found in wheat bran, whole-grain wheat and wheat germ are calculated as follows: $\%=($ minimum + maximum $) / 2$

${ }^{\text {a }}$ Expressed as $\mathrm{g} / 100 \mathrm{~g}$ food

${ }^{\mathrm{b}}$ No data found

${ }^{\mathrm{c}}$ Total free glutathione is given as glutathione equivalents=reduced glutathione $+($ oxidized glutathione $\mathrm{x} 2$ )

${ }^{\mathrm{d}}$ Dietary fibre content is measured according to the AOAC method

${ }^{\mathrm{e}}$ Oligosaccharides include fructons, raffinose and stachyose 
The contents of certain bioactive components present in wheat, rye, oat and barley cereal grains are given in Table 1.2. ${ }^{31}$ Cultivar and growing region may affect the content of the bioactive components that are present. The components presented in Table 1.2 have been reported having physiological functions in the human body, with dietary fiber appearing to act as a carrier in some cases. ${ }^{32}$

Table 1-2 Major bioactive components ( $\mu \mathrm{g} / \mathrm{g}$ dry matter) contained in whole grain wheat, rye, oats, and barley, adopted from Frolich et al. ${ }^{31}$

\begin{tabular}{lcccc}
\hline \multicolumn{1}{c}{ Component } & Wheat & Rye & Oats $^{\mathrm{a}}$ & Barley $^{\mathrm{a}}$ \\
\hline Phytic acid & $390-1,350$ & $540-1,460$ & $420-1,160$ & $380-1,116$ \\
Tocols & $28-80$ & $44-67$ & $16-36$ & $46-69$ \\
Phenolic acids & $326-1,171$ & $491-1,082$ & $351-873$ & $254-675$ \\
Phytosterols & $670-960$ & $1,098-1,420$ & $618-682$ & $899-1,153$ \\
Alkylresorcinols & $220-650$ & $797-1,231$ & Not present & $32-103$ \\
Avenantramides & Not present & Not present & $42-91$ & Not present \\
\hline
\end{tabular}

${ }^{\mathrm{a}}$ Husked and naked cultivar

\subsection{Alkylresorcinols structure and sources}

ARs belong to a large group of phenolic lipids that differ mainly by the oddnumbered hydrocarbon chain attached to position 5 on the benzene ring. ${ }^{33}$ Structurally, ARs consist of 1, 3-dihydroxy-5-alkylbenzene (5-alkylresorcinol) which is alkylated at position 5 by a saturated, odd numbered hydrocarbon side-chain containing 13-27 carbon atoms as shown in Figure 1.2. Depending on the source of the ARs, the structure can vary according to the degree of unsaturation, chain length, ring or chain substituted functional groups on the alkyl chain or aromatic ring, and position of the alkyl chain. ${ }^{33,34}$ These isoprenoid phenolic lipids are present as mixtures of several homologues and derivatives. 
The 5-alkyresorcinols are commonly given abbreviations which describe the alkyl chain length and the degree of unsaturation. For instance, C19:0, C19:1, and C19:2 indicate a saturated chain of 19 carbon atoms, an unsaturated chain of 19 carbons with one double bond, and an unsaturated chain of 19 carbons with two double bonds, respectively. These abbreviations do not carry any indication of the double bond-location. Generally, saturated 5-n-alkylresorcinol homologues ranging in chain length from C15:0-C25:0 are most common in cereal grains. ARs with wide structural variety are found in various plant families and include 5-n-alkyl-, 5-alkenyl-, 5-oxoalkyl-, 5-oxoalkenyl and 5hydroxyalkylresorcinols (Table 1.3). ${ }^{35,36}$

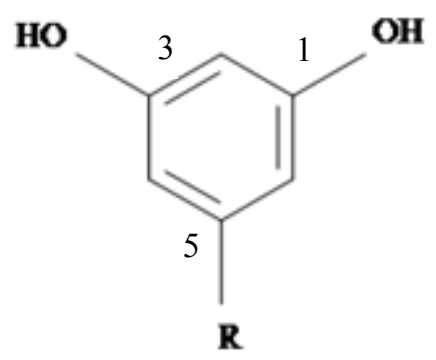

$$
\mathbf{R}=\left(\mathrm{CH}_{2}\right)_{n}\left(\mathrm{CH}_{3}\right)
$$

$$
\boldsymbol{n}=14,16,18,20,22,24
$$

1,3-dihydroxy-5-alkylbenzene (5-alkylresorcinol)

Figure 1-2 The 5-n-alkylresorcinol structure, modified from Ross et al. ${ }^{34}$ 
Table 1-3 Various 5-n-alkyl, 5-alkenyl-, 5-(oxoalkyl)-, 5-(oxoalkenyl)-, and 5(hydroxylalkenyl)-resorcinols isolated from wheat and rye, adopted from Ross et al. ${ }^{34}$

\begin{tabular}{|c|c|c|}
\hline Alkylresorinol derivative $(\mathrm{R})$ & Structure & Molecular weight (Da) \\
\hline \multicolumn{3}{|l|}{ 5-n-Alkylresorcinols } \\
\hline $\mathrm{C} 17: 0$ & 5-n-Heptadecylresorcinol & 348 \\
\hline C19:0 & 5-n-Nonadecanylresorcinol & 376 \\
\hline $\mathrm{C} 21: 0$ & 5- $n$-Heneicosylresorcinol & 404 \\
\hline $\mathrm{C} 23: 0$ & 5-n-Tricosylresorcinol & 432 \\
\hline $\mathrm{C} 25: 0$ & 5-n-Pentacosylresorcinol & 480 \\
\hline \multicolumn{3}{|l|}{ 5-n-Alkenylresorcinols } \\
\hline $\mathrm{C} 17: 1$ & 5-(Heptadecenyl)-resorcinol & 346 \\
\hline C19:1 & 5-(Nonadecenyl)-resorcinol & 374 \\
\hline $\mathrm{C} 21: 1$ & 5-(Heneicosenyl)-resorcinol & 402 \\
\hline $\mathrm{C} 23: 1$ & 5-(Triocosenyl)-resorcinol & 430 \\
\hline $\mathrm{C} 25: 1$ & 5-(Pentacosenyl)-resorcinol & 458 \\
\hline C19:2 & 5-(Nona-10Z, 13Z-decadienyl)-resorcinol & 372 \\
\hline $\mathrm{C} 21: 2$ & 5-(Henei-12Z, 15Z-cosadienyl)-resorcinol & 400 \\
\hline $\mathrm{C} 23: 2$ & 5-(Tri-14Z, 17Z-cosadienyl)-resorcinol & 428 \\
\hline $\mathrm{C} 25: 2$ & 5-(Penta-16Z, 19Z-cosadienyl)-resorcinol & 456 \\
\hline \multicolumn{3}{|l|}{ 5-Oxoalkylresorcinols } \\
\hline C19:Oxo & 5-(2-Oxononadecanyl)-resorcinol & 390 \\
\hline C21:Oxo & 5-(2-Oxoheneicosanyl)-resorcinol & 418 \\
\hline C23:Oxo & 5-(2-Oxotriocosanyl)-resorcinol & 446 \\
\hline C25:Oxo & 5-(2-Oxopentacosanyl)-resorcinol & 474 \\
\hline \multicolumn{3}{|l|}{ 5-Oxoalkenylresorcinols } \\
\hline C19:1, Oxо & 5-(2-Oxononadecenyl)-resorcinol & 388 \\
\hline C21:1, Oxо & 5-(2-Oxoheneicosenyl)-resorcinol & 416 \\
\hline C23:1, Oxo & 5-(2-Oxotriocosenyl)-resorcinol & 444 \\
\hline C25:1, Oxо & 5-(2-Oxopentacosenyl)-resorcinol & 472 \\
\hline C19:2, Oxo & 5-(2-Oxo,-10Z, 13Z-nonadecadienyl)-resorcinol & 386 \\
\hline $\mathrm{C} 21: 2$, Oxo & 5-(2-Oxo,12Z,15Z-heneicosenyl)-resorcinol & 414 \\
\hline $\mathrm{C} 23: 2$, Oxо & 5-(2-Oxo,14Z,17Z-tricosadienyl)-resorcinol & 442 \\
\hline $\mathrm{C} 25: 2$, Oxo & 5-(2-Oxo, 16Z,19Z-pentacosadienyl)-resorcinol & 470 \\
\hline \multicolumn{3}{|l|}{ 5-Hydroxyalkenylresorcinols } \\
\hline C19:1,Hydroxy & 5-(2-Hydroxynonadecenyl)-resorcinol & 390 \\
\hline C21:1,Hydroxy & 5-(2-Hydroxyheneicosenyl)-resorcinol & 418 \\
\hline C21:1,Hydroxy & 5-(4-Hydroxyheneicosenyl)-resorcinol & 418 \\
\hline C23:1,Hydroxy & 5-(4-Hydroxytricosenyl)-resorcinol & 446 \\
\hline C25:1,Hydroxy & 5-(4-Hydroxypentacosenyl)-resorcinol & 474 \\
\hline C21:2,Hydroxy & 5-(4-Hydroxy, 12Z,15Z-heneicosenyl)-resorcinol & 416 \\
\hline C23:2,Hydroxy & 5-(4-Hydroxy, $14 Z, 17 Z$-tricosadienyl)-resorcinol & 444 \\
\hline
\end{tabular}


It has been reported that ARs have been found in eleven plant families including Anacardiaceae, Ginkgoaceae, Proteaceae, Myrsinaceae, Primulaceae, Myristicaceae, Iridaceae, Compositae, Leguminosae and Gramineae. ${ }^{37}$ The amount of ARs present varies largely depending on the plant source. Some plants contain ARs that vary from $0.005 \%(\mathrm{~m} / \mathrm{m}$, mass $/ \mathrm{mass})$ for Hordeum vulgare (barley) grains to $0.3 \%(\mathrm{~m} / \mathrm{m})$ for Secale cereale (rye) grains. ${ }^{37,38}$ The highest concentration of ARs has been reported in the oil extract of cashew nut shells containing up to $20 \%(\mathrm{~m} / \mathrm{m})$ of ARs. ${ }^{33}$

The existence of both a water soluble polar 'head' (dihydroxybenzene group) and a water insoluble non-polar alkyl 'tail' gives ARs their amphiphilic nature. This is of great significance with regards to their analysis, absorption, metabolism and potential bioactivity. ${ }^{33}$ The octanol/water partition coefficients $\left(\log \mathrm{P}_{\mathrm{o} / \mathrm{w}}\right)$ for ARs are between 7.0 and 13.4, and the hydrophobicity increases with increasing alkyl chain length. ${ }^{34,39}$

Although they can be isolated from a wide variety of sources, ARs in edible food sources provide the greatest dietary advantage with the essential dietary intake of ARs coming from foods containing whole-grain rye or wheat kernels. ${ }^{34,38}$ There has been a large amount of research reporting the potential positive effects of ARs on humans. ${ }^{40}$ However, the initial interest in ARs started decades ago from concerning findings that suggested ARs in cattle feed caused inhibition of growth and decrease of appetite in animals. This led to the need and development of a rapid method for determining the presence of ARs. ${ }^{40,41}$

ARs are detected during kernel development in cereals, and the amount has been found to decrease as the grain matures. ${ }^{42}$ This decrease might be due to dilution effect caused by the increase in proportion of starch and protein in the maturing grain kernel. 
ARs have the ability to protect plants from mold and other harmful organisms such as bacteria, which attack from the outside. The location of ARs in the outer layers (in the bran of cereal grains) enhances these protective effects. ${ }^{43} 44$ The exact location of ARs in a cereal kernel has been determined to be in the intermediate layer between the testa and pericarp, with this layer containing $99 \%$ of the ARs. ${ }^{45}$

\subsection{Alkylresorcinols in cereals}

The highest levels of ARs are found in rye ${ }^{46}$, wheat ${ }^{47}$, and triticale ${ }^{46}$, with the lowest levels being reported in barley. ${ }^{46,48}$ Although trace amount of ARs have been reported in rice, millet, oats, corn, and sorghum ${ }^{34-36,45,46,48,49}$, other studies found no ARs

present in these grains. ${ }^{36,46,50}$ The AR contents of whole cereal grains, the bran fraction, and the flour is shown in Table 1.4.

Table 1-4 Alkylresorcinol content of whole cereal grains, bran and flour ${ }^{35,45,46}$

\begin{tabular}{lcc}
\hline Cereal & Fractions & Range $(\mu \mathrm{g} / \mathrm{g}$, dry matter $)$ \\
\hline \multirow{3}{*}{ Rye } & Whole grain & $560-1444$ \\
& Bran & $2400-4108$ \\
& Flour & $69-79$ \\
Wheat & Whole grain & $264-943$ \\
& Bran & $2210-3225$ \\
& Flour & $29-45$ \\
Triticale & Whole grain & $430-700$ \\
& Bran & $2780-3080$ \\
Barley & Whole grain & $41-74$ \\
& Bran & 210 \\
\hline
\end{tabular}

The AR content is reported to vary widely within and between species due to environmental, agricultural and genetic factors. ${ }^{51-53}$ Normally, AR contents in the bran 
fractions of a given cereal are two to five times greater than its respective whole grain ARs content. ${ }^{36,46}$ The ratio of AR homologue C17:0/C21:0 is unique for each of the cereal grains, and is approximately 0.2 in triticale, 0.1 in wheat, and 1.0 in rye bran. ARs are of interest as biomarkers of whole grain cereal intake, and as such are an aid in understanding the link between whole grain consumption and health. ${ }^{54}$

The Figure 1.3 shows the varying amounts of each of the AR homologues in the

different cereal grains: rye, common wheat, durum wheat, triticale, and barley. ${ }^{34,49,55}$ Each grain has predominant homologues specific to that grain type. The most abundant AR homologues in rye, common wheat, durum wheat, triticale, and barley are C19:0, $\mathrm{C} 21: 0$, and $\mathrm{C} 25: 0$. 


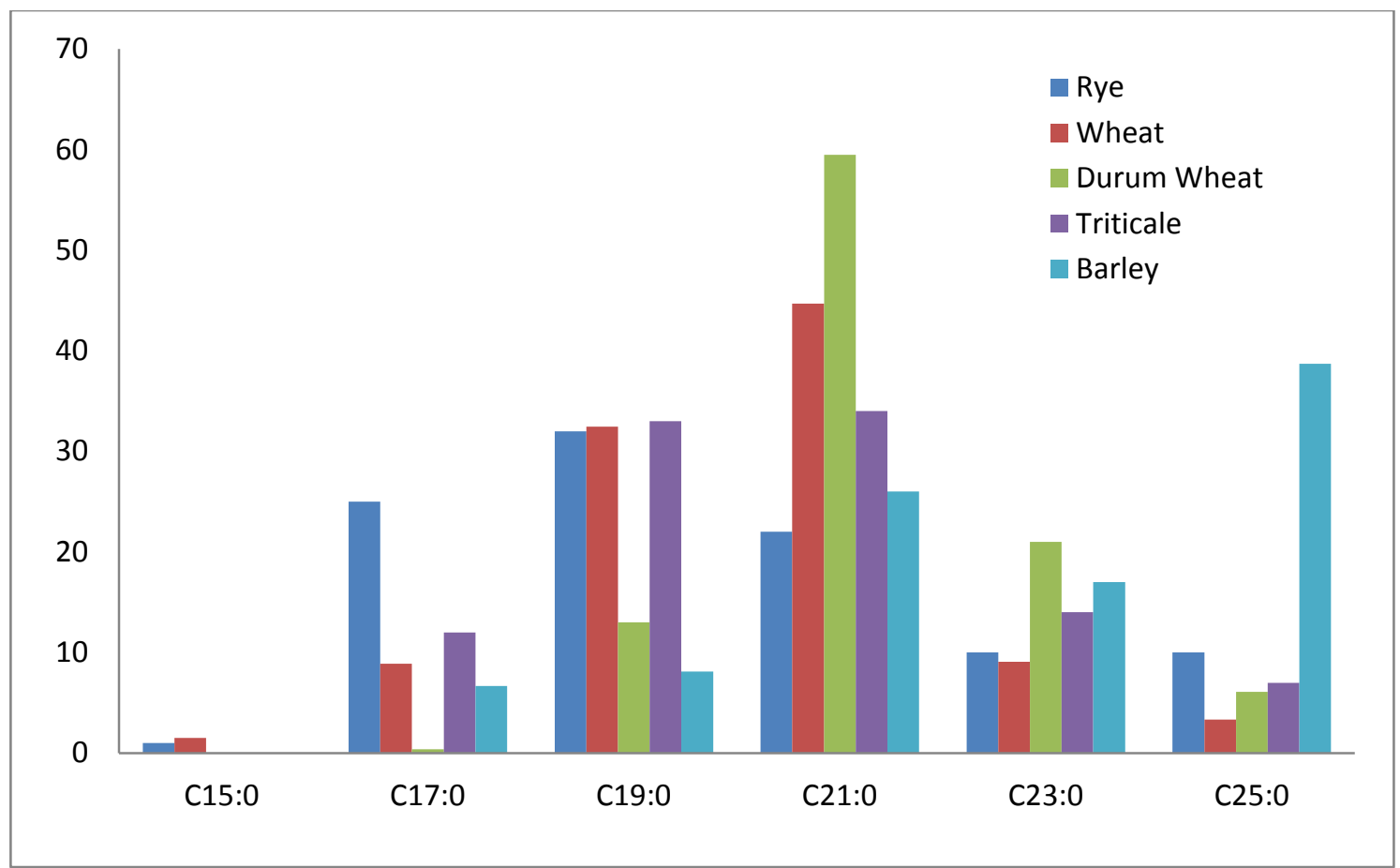

Figure 1-3 Typical percent distribution of the alkylresorcinol homologues C15:0-C25:0 found in rye, ${ }^{46}$ common wheat, ${ }^{47}$ durum wheat, ${ }^{55,56}$ triticale, $^{46}$ and barley, ${ }^{46,48}$ modified from Söderholm ${ }^{57}$

\subsection{Biosynthesis of alkylresorcinols}

Plant phenolic compounds are synthesized from two main pathways: the shikimate pathway and the polyketide (acetate) pathway. The shikimate pathway forms phenylpropanoids such as the hydroxycinnamic acids and coumarins, and the polyketide pathway produces simple phenols which lead to the formation of quinones. ${ }^{33}$ The largest groups of phenolics (flavonoids) are formed through a combination of both pathways.

The biosynthesis of resorcinolic lipids (a form of phenolic lipids) are believed to be derived from the polyketide (acetate) pathway by 2 -oxoalkylresorcylic acid synthase (ORAS), a type III polyketide synthase. ${ }^{58}$ Polyketide biosynthesis begins with polyketomethylenic chains, $-\left(\mathrm{CH}_{2}-\mathrm{C}\right)_{m}{ }^{-}$, derived from acetic acid and the activated forms 
of acetyl-S-CoA and malonyl-S-CoA. ${ }^{59}$ The biosynthesis pathway for polyketides resembles the pathway for fatty acids. For both of them, the formation of linear chains occurs by the addition of $\mathrm{C}_{2}$ units. A fatty acyl-coA reacts with malonyl coenzyme A three times to form a tetraketide intermediate by an aldol condensation-type reaction. ${ }^{60}$ The alkyl side chain (the odd numbered side chain) of ARs is the direct result of the fatty acid precursor. ${ }^{61}$ The intermediate compound undergoes an aldol condensation-type ring closure and hydrolysis to release the coeznyme A to form alkylresorcylic acid. The next step is non-enzymatic decarboxylation to yield 5-n-alkylresorcinols. ${ }^{43}$ ORAS synthesizes triketide and tetraketide pyrones as well as pentaketide resorcylic acids and resorcinols from the fatty acyl-CoA starter unit (Figure 1.4). ${ }^{58}$ 


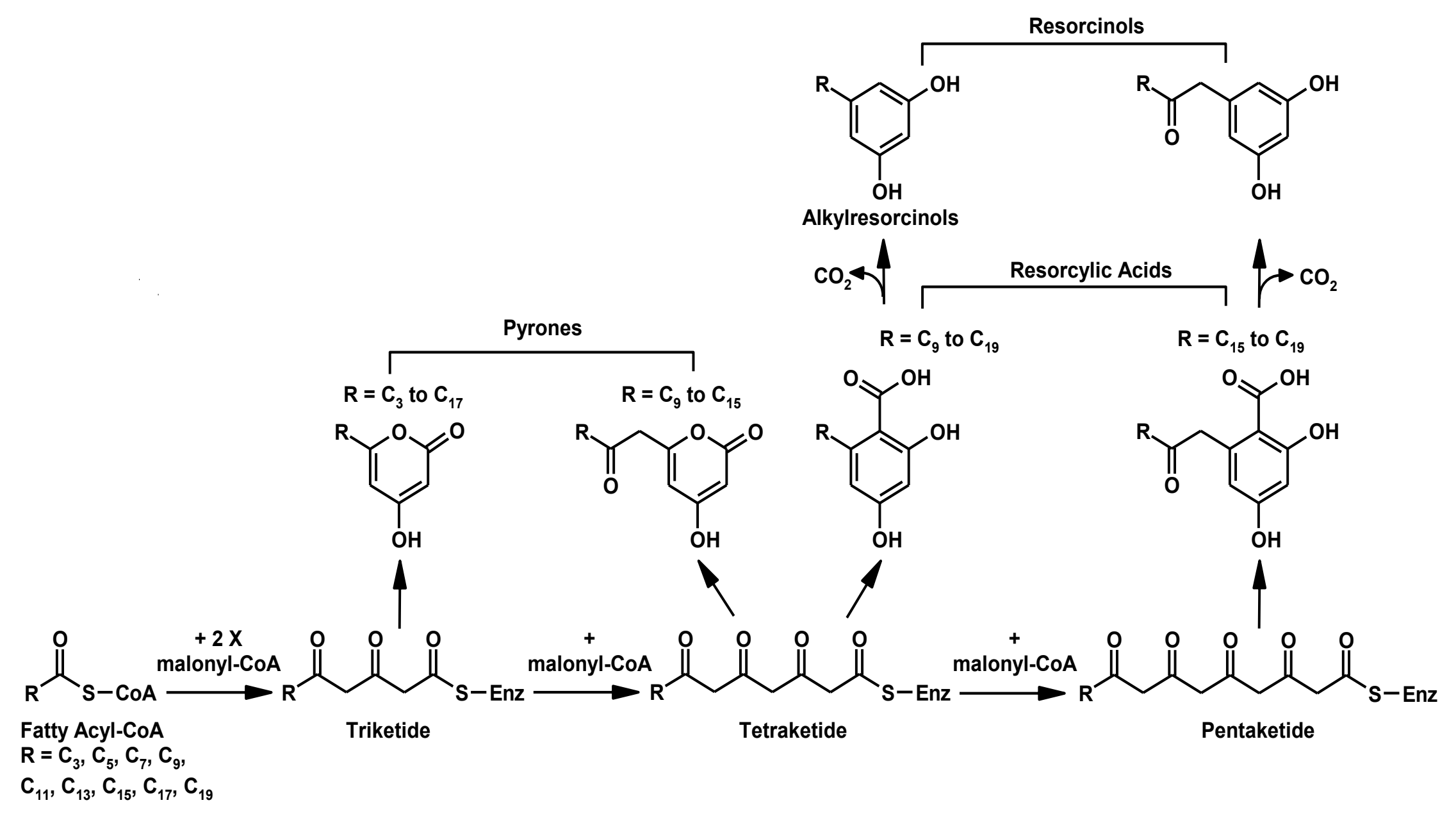

Figure 1-4 The biosynthesis of alkylresorcinols and various polyketide (triketide, tetraketide and pentaketide) resorcylic acids, adopted from Yu et al. ${ }^{58}$ 


\subsection{Extraction of alkylresorcinols}

Different solvent extraction methods are commonly used for the extraction of ARs from cereals using organic solvents such as acetone, ethyl acetate, and methanol. However, using acetone and ethyl acetate are not suitable for the extraction of ARs from bread. ${ }^{46}$ For this purpose, a hot-1-propanol: water $(3: 1, v / v)$ solution is used for the extraction and complete recovery of ARs from baked products. ${ }^{46}$

It was believed that baking destroyed or reduced the ARs content in bread, since only low amounts of ARs were detected in bread. ${ }^{50,62}$ Later experiments showed that ARs remain stable during baking and food processing. ${ }^{38,56,63}$

ARs are amphiphillic compounds that become more water insoluble with increasing alkyl chain length. ${ }^{33}$ For this reason, cereal grain ARs have been traditionally extracted using organic solvents, especially acetone, and ethyl acetate, at room temperature for 16-24 $\mathrm{h}$ with a sample to organic solvent ratio between 1:40 and 1:50 (w/v). ${ }^{34}$ There are several disadvantages to using the organic solvent extraction methods, including long extraction time, toxic waste generation, and lack of selectivity. Additionally, there is concern for food security, safety, and quality when organic solvents are used. ${ }^{64}$ Supercritical carbon dioxide $\left(\mathrm{SC}-\mathrm{CO}_{2}\right)$ extraction can be used as an alternative to the traditional solvent extraction methods as explained in the following section.

\subsection{Supercritical carbon dioxide $\left(\mathrm{SC}-\mathrm{CO}_{2}\right)$ extraction of ARs}

Supercritical fluid technology has gained increased interest as a green technology, especially for use in the food industry. Fluids are put into the supercritical phase by increasing the pressure and temperature above the critical point. Once in the supercritical 
phase, fluids have liquid-like solvent power and gas-like diffusivity. ${ }^{65}$ Carbon dioxide $\left(\mathrm{CO}_{2}\right)$ is the most widely used supercritical fluid, and when heated and compressed above its critical point, $\mathrm{SC}-\mathrm{CO}_{2}$ is produced. Figure 4.1 in Chapter 4 shows the $\mathrm{SC}-\mathrm{CO}_{2}$ instrument in our research laboratory at Carleton University

$\mathrm{SC}-\mathrm{CO}_{2}$ can be used as an extraction solvent to extract the ARs, and the efficiency of this extraction method depends on the pressure, temperature, and co-solvent used. ${ }^{66}$ The $\mathrm{SC}-\mathrm{CO}_{2}$ extraction technique is very attractive in comparison to traditional organic solvent procedures because of its non-toxic, non-flammable, low-cost, and environmentally friendly properties. ${ }^{35,66}$ Additionally, it has a faster extraction time than the traditional techniques and the $\mathrm{CO}_{2}$ is safe and easy to remove after the extraction is complete. The use of $\mathrm{SC}-\mathrm{CO}_{2}$ technology has been well established for the extraction of many different food products, including essential oils. ${ }^{67}$ It has also been used to extract ARs from wheat and rye bran at the optimum conditions of $70{ }^{\circ} \mathrm{C}$ and $35 \mathrm{MPa}$ with a flow rate of $25 \mathrm{~g} / \mathrm{min}$ for $4 \mathrm{~h}$ followed by using $10 \%$ ethanol as a co-solvent/modifier. ${ }^{35}$ Because of its non-polar nature, pure $\mathrm{CO}_{2}$ does not solubilize the polar ARs. For that reason, ethanol has been suggested as a co-solvent when using $\mathrm{SC}-\mathrm{CO}_{2}$ for the removal of ARs from wheat and rye bran. Previous studies have found $\mathrm{SC}-\mathrm{CO}_{2}$ to yield $8-80 \%$ more ARs than when traditional extraction was done using acetone. ${ }^{66}$ However, some recent studies have found there are no significant differences in the total AR yield between the two methods. $^{35,68}$

\subsection{ARs analysis methods}

In literature, many studies have described the isolation of ARs from different plant matrices and the analysis of extracts by different techniques. These studies and the 
chromatographic techniques used in AR analysis have been comprehensively reviewed..$^{33,34}$

ARs in rye were reported in 1967 as a mixture of homologues C15:0-C25:0. Since then, the analysis and isolation of ARs in cereal grains have been performed using a variety of techniques. ${ }^{69}$ A number of analytical methods have been employed to quantify AR concentration in plasma such as gas chromatography/mass spectrometry (GC-MS), ${ }^{70}$ gas chromatography/tandem mass spectrometry), ${ }^{71}$ and liquid chromatography/tandem mass spectrometry. ${ }^{72}$ The following sections include detailed information about determination techniques.

\subsubsection{Colorimetric determination of ARs}

ARs were first thought to be growth inhibitors for the livestock that were fed with the rye, causing lower weight gain compared to animals fed with other cereal grains. This initiated screening of the rye cultivar and lines using colorimetric studies to determine which had the lowest possible concentrations of ARs. ${ }^{40,69}$ A colorimetric analysis method has been used in several studies to quantify the total ARs content by measuring the intensity of the electromagnetic radiation in the visible spectrum that is transmitted through an AR extract. ${ }^{53,73-75}$ This method is based on a diazonium salt reacting with phenolic compounds to form an azo-complex which develops a reddish violet colour with a maximum absorption peak at $520 \mathrm{~nm}$ distinct for ARs. ${ }^{75,76}$ There have been various diazotized compounds used as reagents for the method, including Fast Blue B BF4, ${ }^{47,74}$ Fast Blue B Zn ${ }^{70,75}$ and Fast Blue RR salt. ${ }^{70}$ However, the colorimetric method does not differentiate between the various $\mathrm{AR}$ homologues present in the extract as it only quantifies based on the number of aromatic rings that react with the diazonium salt. ${ }^{47}$ For 
this reason, the colorimetric method is only good for rapid and cost-effective detection and quantification of the total ARs content. ${ }^{77}$ Further chromatographic analysis is needed for confirmation and differentiation between the different AR homologues. ${ }^{51,78}$

\subsubsection{Thin layer and paper chromatography}

Thin layer chromatography (TLC) and paper chromatography have been used for the identification and separation of ARs qualitatively. ${ }^{45,79-81}$ Cereal grains have been analyzed by TLC using silica gel or alumina on the plates. ${ }^{82-84}$ After running the TLC plate it is stained with a staining reagent such as Fast Blue B to indicate the presence of any ARs. TLC is relatively simple and easy to perform. However, for quantitative determinations, accurate chromatographic techniques must be used.

\subsubsection{High performance liquid chromatography (HPLC)}

High performance liquid chromatography (HPLC) with reverse phase (RP) columns has been used in many studies to analyze the composition of various AR extracts. ${ }^{36,50}$ Identification of AR homologues is based on their characteristic retention times, with these being dependent on the AR-chain length and the degree of unsaturation. ${ }^{37,50,85}$ It has been found that using a gradient elution system ${ }^{36,86}$ gives a faster and better separation than when using an isocratic system. ${ }^{34}, 84$ RP-HPLC with a non-polar $\mathrm{C} 18$ column as the stationary phase and a polar solvent (e.g. methanol) as the mobile phase is generally used to separate ARs. ${ }^{37,50,86}$ Gradient systems produce optimal results with clear peak separation and reduced background noise in the resulting chromatogram. A photodiode array detector (PDA) ${ }^{87,88}$ at $280 \mathrm{~nm}$ has been used for the detection of ARs from cereal bran. ${ }^{80,89}$ 


\subsubsection{GC-MS}

Gas chromatography combined with mass spectrometry (GC-MS) separates and quantifies the relative amounts of the different AR homologues based on their individual retention times which are visualized by the MS detector. It seems to be an efficient method for the determination of ARs content from different matrices. ${ }^{46,86,88}$ Generally a non-polar stationary phase is used in the GC column, with a flame ionization detector (FID) and electron impact (EI) for ionization in the MS stage of the procedure. GC alone is not enough to identify all of the AR components of the extracts. Using GC-MS identifies the AR components with high precision through their characteristic ionized fragments of specific mass to charge $(\mathrm{m} / \mathrm{z})$ ratios and retention times. ${ }^{35,90}$ In GC-MS studies, the ion with $\mathrm{m} / \mathrm{z}$ ratio of 268 is a typical peak for di-trimethylsilylated (TMS) $\mathrm{AR}^{46}$. The characteristic $\mathrm{m} / \mathrm{z}$ ion peaks for each $\mathrm{AR}$ homologues starting from $\mathrm{C} 15: 0$ to $\mathrm{C} 25: 0$ are $320,348,376,404,432$ and 460 respectively. $^{91}$

\subsection{Bioactivity of alkylresorcinols}

ARs have been reported to have various biological activities. Most of the activities of ARs are thought to be based on their ability to interact with biological membranes. For instance, ARs are capable of binding to the hydrophobic regions of some proteins and modulating their enzymatic activity. ${ }^{92}$ The AR homologues C15:0 and C19:0 have been reported to inhibit the activity of glycerol 3-phosphate dehydrogenase at micromolar concentrations. ${ }^{93,94}$ Data concerning other homologs (AR-C21:0-C23:0 and C24:0) are missing. In the following topics some aspects of bioactivity such as antioxidant activity and the measuring methods used to determine this is covered. 


\subsubsection{Free radicals and antioxidant activity}

Oxidative damage is caused by reactive oxygen species (ROS) and reactive nitrogen species (RNS). They are classified as "free radicals" and produced as a byproduct of aerobic metabolism. Cell damage can be caused by an imbalance of free radicals, in which the level of oxidants exceeds that of antioxidants. ${ }^{95}$ Free radicals are believed to contribute to several inflammatory and degenerative diseases. ${ }^{96}$ Some dietary antioxidants include radical chain reaction inhibitors, metal chelators, oxidative enzyme inhibitors, and antioxidant enzyme cofactors. ${ }^{97}$ There is a link between increased levels of lipid oxidation products and diseases such as diabetes and atherosclerosis. ${ }^{98,99}$ For example, oxidized cholesterol of fatty acid moieties in plasma LDL can lead to the development of atherosclerosis. ${ }^{98}$

Phenolic antioxidants are an important class of compounds for inhibiting the oxidation of materials for both commercial and biological importance. ${ }^{100}$ An antioxidant is defined as a substance in foods that when present at low concentrations compared to those of an oxidizable substrate significantly decreases or prevents the adverse effects of reactive species, such as ROS and RNS, in the normal physiological function of humans. ${ }^{97,101}$ However, not all reductants involved in a chemical reaction are antioxidants. Only those compounds capable of protecting the biological target from oxidants meet this principle. ${ }^{102}$

There are several mechanisms of actions for antioxidants such as a) serving as physical barriers to prevent ROS generation such as a UV filter or cell membranes, b) chemical traps that quench ROS by absorbing energy and electrons, such as carotenoids, and anthocyanins, c) catalytic systems that neutralize ROS, for instance, the antioxidant enzymes superoxide dismutase (SOD), catalase, and glutathione peroxidase ${ }^{103}$, d) 
binding/inactivation of metal ions to prevent ROS generation by ferritin, catechins, and by chain-breaking antioxidants such as ascorbic acid, tocopherols, and flavonoids. ${ }^{104}$

There are two pathways for oxidation in which antioxidants can play a preventive role including $\mathrm{H}$-atom transfer (HAT) and single electron transfer (SET). The HAT pathway of lipid peroxidation is illustrated below: ${ }^{100}$

$$
\begin{array}{ll}
\mathrm{RH} \longrightarrow \mathrm{R}^{\bullet} & \text { (initiation) } \\
\mathrm{R}^{\bullet}+\mathrm{O}_{2} \longrightarrow \mathrm{ROO}^{\bullet} & \text { (addition of } \left.\mathrm{O}_{2}\right) \\
\mathrm{ROO}^{\circ}+\mathrm{RH} \longrightarrow \mathrm{ROOH}+\mathrm{R}^{\bullet} & \text { (H-atom transfer) } \\
\mathrm{ROO}^{\circ}+\mathrm{AH} \longrightarrow \mathrm{ROOH}+\mathrm{AO}^{\bullet} &
\end{array}
$$

Once a free radical $\left(\mathrm{R}^{\circ}\right)$ has been generated, a chain reaction is created by the reactions 2 and 3. Through these chain cycles, many lipid molecules (R-H) are converted into lipid hydroperoxide $(\mathrm{ROOH})$, resulting in oxidation and rancidity of fats. The role of an antioxidant such as phenolic antioxidants (AH), is to interrupt the chain reaction. ${ }^{100}$

An effective AH must be a relatively stable free radical and react slowly with substrate $(\mathrm{RH})$ but quickly with hydroxyperoxide (ROO*). The most effective lipidsoluble chain-breaking antioxidant in human blood plasma is known as $\alpha$-tocopherol, the most active component of Vitamin E. ${ }^{105}$

The rate of reaction of substrate RH with peroxyl radicals depends on the barrier height for transfer of an $\mathrm{H}$-atom from $\mathrm{AH}$. The bond dissociation enthalpy (BDE) ${ }^{106}$ in phenolic antioxidants is an important factor in determining the efficacy of an antioxidant, 
since the weaker the $\mathrm{OH}$ bond the faster will be the reaction with free radicals. ${ }^{100}$ The $\mathrm{BDE}$ of phenol provides a reference value for all phenolic antioxidants, and Wright et al. (2001) has studied the procedures for estimating the O-H BDE based on group additivity rules and tested on several classes of phenolic antioxidants including commercial ones used as food additives. On the basis of their study results, they were able to interpret relative rates for the reaction of antioxidants with free radicals, including a comparison of both HAT and SET mechanism in which HAT was dominant in most cases. ${ }^{100}$

In the work ${ }^{92}$ by Kozubek and Nienartowicz, it was found that ARs decreased peroxidation products at micromolar levels in suspensions of erythrocytes injected with hydrogen peroxide and homologs of ARs. The homolog AR-C15:0 had the highest antioxidant effect followed by $\mathrm{C} 19: 0$, and $\mathrm{C} 23: 0$. From this study it was suggested that the antioxidant potential of AR homologs is dependent on their chain length, with the ARs with shorter alkyl chain lengths having higher antioxidant activity. ${ }^{92}$ In another study ${ }^{107}$, Winota and Lorenz showed that AR homolog-C15:0 slowed the rate of oxidative rancidity in oil as well as lipid oxidation in cereal products at AR concentrations of $0.050-0.075 \%$. These findings suggest that ARs might be useful as natural antioxidants in the food industry. ${ }^{108}$

In conclusion, many studies shown that ARs from cereal bran isolates serve as antioxidants in food and biological systems. However, detailed information of about how ARs act in the membranes, in different emulsion or food systems, the potential of ARs to act as preventive antioxidants, and if any synergistic effect of ARs exist, are only a few of the many questions that remain to be answered in regards to the antioxidant activity of ARs. ${ }^{109}$ 


\subsubsection{Oxygen radical absorbance capacity (ORAC)}

The peroxyl radical is an important physiological radical. It is involved in the propagating steps in lipid peroxidation chain reactions. They can be formed in vivo through reactions of carbon centered radicals with other reactive oxygen species like peroxyl radicals. The ORAC assay measures the inhibition of peroxyl-radical-induced oxidants and reflects classical radical chain breaking antioxidant activity by $\mathrm{H}$-atom transfer. ${ }^{110}$ Basically, peroxyl radicals are produced from thermal decomposition of AAPH (2, 2'-azobis (2-amidino-propane) dihydrochloride) and react with a fluorescent probe (oxidizable protein substrate) to form a nonfluorescent product that can be quantitated by fluorescence. ${ }^{111}$

The ORAC value for an antioxidant is calculated based on the areas under the reaction kinetic curves for the antioxidant sample, antioxidant standard, and the blank. Trolox is generally used as the antioxidant standard, and results are expressed as in micromoles of trolox equivalents per unit of the sample ( $\mu$ mole TE/g). ${ }^{112}$

The ORAC assay has several advantages compared to other antioxidant scavenging capacity assays such as $\mathrm{ABTS}^{\cdot+}$ and $\mathrm{DPPH}^{\circ}$. For instance, it measures scavenging activity against a physiological relevant radical which is peroxyl radical, and it is well known that it plays a role in the oxidation of lipids in human bodies and food systems. ${ }^{97}$ Also, the assay working conditions are conducted under physiological $\mathrm{pH}$ and for high throughput analysis. Lastly, the assay takes into consideration both kinetic and thermodynamic properties of antioxidant-radical reactions. ${ }^{112}$ 


\subsubsection{Diphenyl-picryhydrazyl (DPPH)}

This assay has found extensive use in screening the antioxidant properties of pure compounds and botanical extracts. It is technically simple and can be performed using a UV-Vis spectrophotometer. ${ }^{113}$ The 2, 2-diphenyl-1-picryhydrazyl (DPPH) radical is a long-lived organic nitrogen radical and has a deep purple color. The purple chromogen radical is reduced by antioxidants to the corresponding pale yellow hydrazine and the reducing power of the antioxidants is measured by monitoring the absorbance decrease at

515-528 nm. This method was first reported by Brand-Williams et al. ${ }^{14}$ The main advantage of this assay over other antioxidant capacity measurement assays is its broad solvent compatibility with aqueous, polar, and non-organic solvents. ${ }^{113}$ The calculated percent DPPH remaining is proportional to the antioxidant concentration, and the $\mathrm{EC}_{50}$ is defined as the effective concentration that causes a 50\% decrease in the initial DPPH amount. $^{114}$

\subsection{Prebiotic activity}

The definition of dietary fiber (DF) by AACC (the American Association of Cereals Chemist) includes "cell wall polysaccharides, lignin and associated substances resistant to hydrolysis by the digestive enzymes of humans". Most of those mentioned "conjugated compounds" are related dietary antioxidants such as phenolic acids. The most abundant phenolic compound in wheat is ferulic acid (Figure 5) followed by sinapic acid, p-coumaric acid, and caffeic acid. ${ }^{115,116}$ Most grain polyphenolic compounds $(\sim 95 \%)$ are linked to cell wall polysaccharides. Those dietary fiber-phenolic compounds are covalently bound to polysaccharides through ester bonds. For instance, ferulic acid (Figure 5) is bound to the arabinoxylans via the acid group acylating the primary 
hydroxyl at the C5 position of $\alpha$-arabinofuronasyl residues (Figure 6). ${ }^{117}$ They might be hydrolyzed through wheat related endoxylanases or specific intestinal bacteria possessing arabinoxylan-degrading enzymes. This hydrolysis leads to the formation of arabinoxylan oligosaccharides (AXOS), representing a new class of candidate prebiotics. Little information is currently available on the prebiotic potency of AXOS in vivo. ${ }^{118}$ 


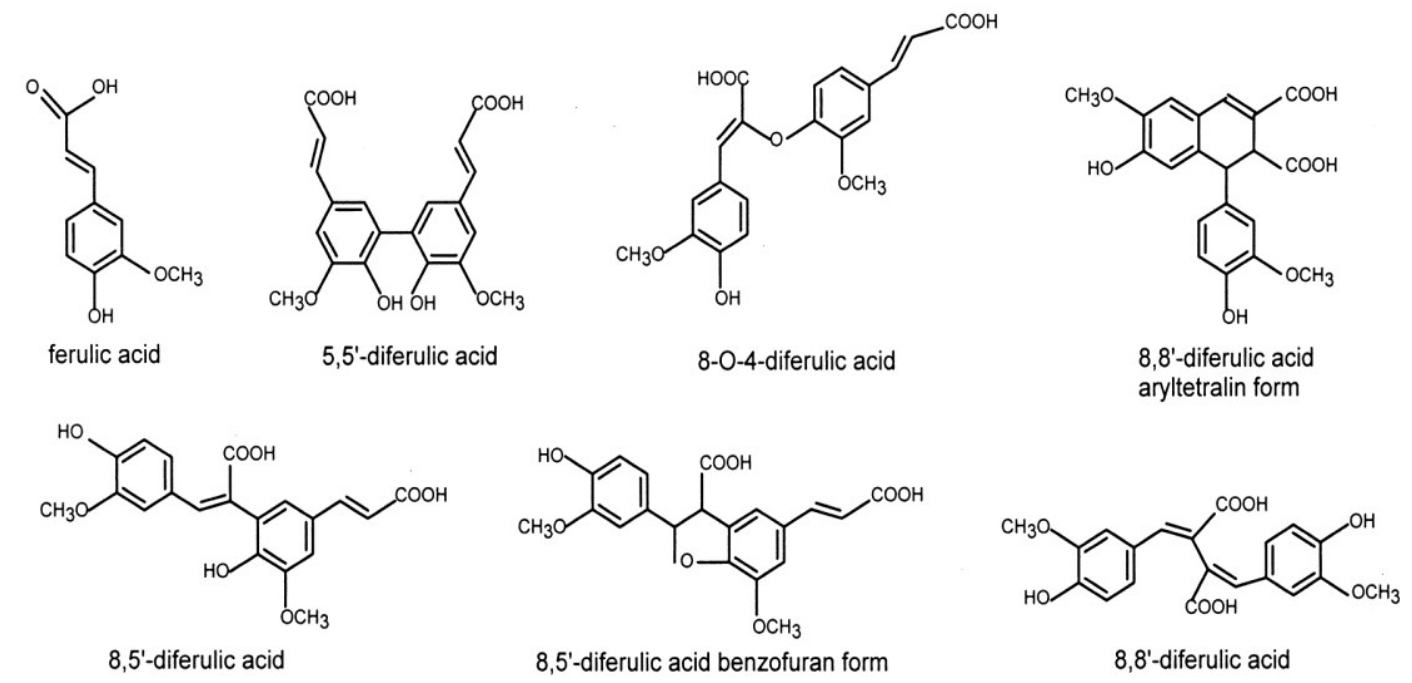

Figure 1-5 Chemical structures of ferulic acid and diferulic acid identified in plant cell walls, adopted from de Vries et al. ${ }^{119}$

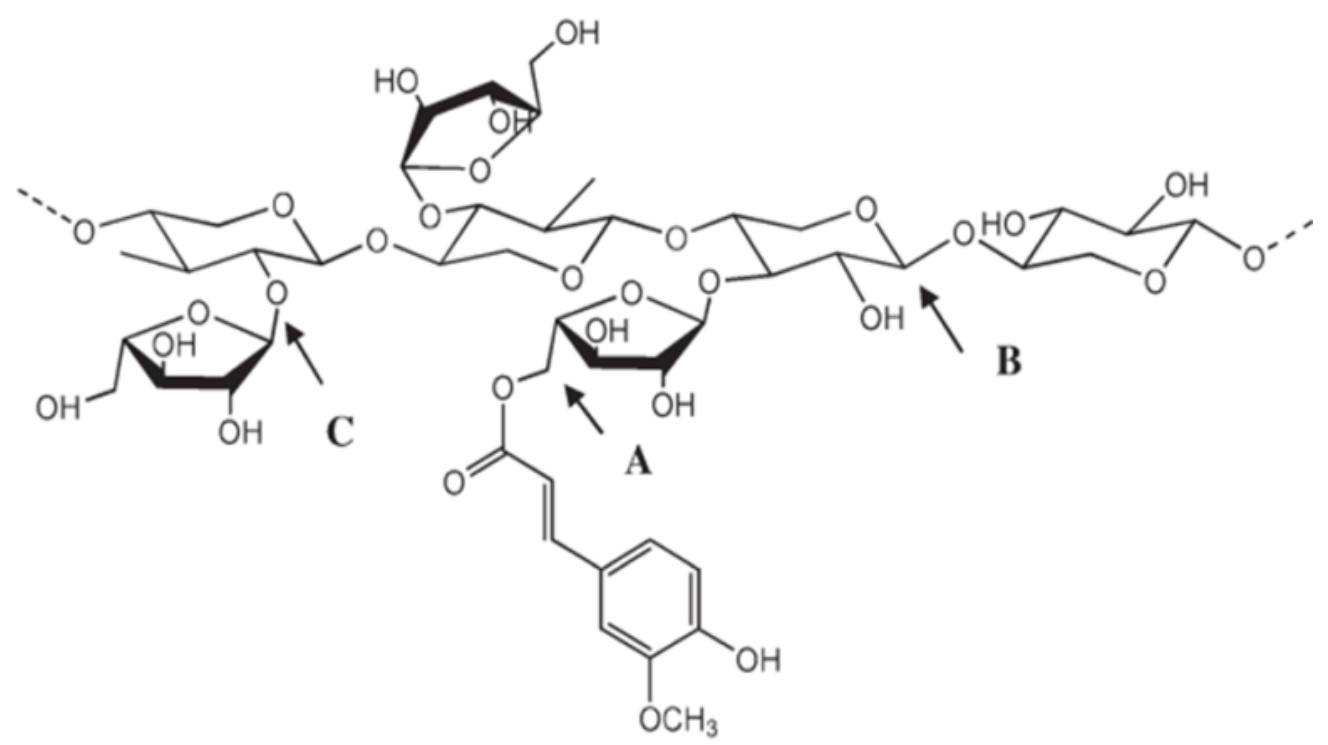

Figure 1-6 The structure of ferulic acid esterified to units of arabinoxylan. A: ferulic acid linked to $0-5$ arabinose chain of arabinoxylan; B: $\beta-1,4-$ linked xylan backbone; C: $\alpha$ 1,2-linked L-arabinose. ${ }^{117}$ 
DF can also be used as a source of prebiotics. Prebiotics are defined as nondigestible food ingredients such as fructooligosaccharides (FOS), inulin, galactooligosaccharides and other related carbohydrates that beneficially affect the host by stimulating the growth, or activity of one or a limited number of bacteria such as Lactobacilli and Bifidobacteria. ${ }^{120}$

It has been reported that only the intestinal microflora is able to disrupt the DF matrix and release the associated AOX in soft physiological conditions (neutral $\mathrm{pH}, 37$ $\left.{ }^{\circ} \mathrm{C}\right)$. Also, the human microflora can hydrolyze, reduce, decarboxylate, demethylate, and dehydroxylate polyphenols (PP), and produce several metabolites. ${ }^{121}$ The colon is a complex ecosystem with more than 400 different species of bacteria (>95\% concentrated in the colon). PP are metabolized by gut microbiota and produce metabolites such as hydroxyphenylacetic, phenylpropionic and phenylbutric acids with potential health benefits to the host. ${ }^{122}$ As a result, the bacterial species in the human gut have important metabolic and immune functions. For instance, short chain fatty acids, which are the metabolites from DF, can act as prebiotics and increase the population of beneficial colonic bacteria. DF polyphenols and their metabolites can also inhibit the growth of pathogenic bacteria such as Staphylococcus aureus, Escherichia coli, and Candida albicans. Current findings on these intestinal health effects of polyphenols is based on in vitro and animal studies, however, confirmation in humans is needed. ${ }^{123,124}$ 


\subsection{Thesis objectives}

This thesis was undertaken as a project within the Ontario Ministry of Agriculture, Food, and Rural Affairs (OMAFRA). OMAFRA works as a catalyst for transforming agriculture, food sectors, and rural communities for a healthier Ontario and is committed to protecting the Ontario environment.

This study aimed to characterize ARs in wheat bran, investigate the influence of environmental factors on AR composition, measure AR bioactivity including antioxidant activity and investigate AR stability during baking. Moreover, the prebiotic potential of WB-soluble dietary fibre (SDF) was explored using yogurt models. Specific objectives were achieved by the following:

1. To extract and characterize wheat bran ARs

- Extractions by both traditional and $\mathrm{SC}-\mathrm{CO}_{2}$ methods

- Characterizations of ARs by GC-MS and HPLC-PDA

2. To investigate environmental factors on wheat bran-ARs content and composition

- Effects of region and cultivar on ARs

- Effects of region and cultivar on antioxidant activity

3. To determine the stability of ARs at high temperatures using baking as a food model

- AR content and composition before and after baking

- Antioxidant activity before and after baking

4. To evaluate prebiotic activity of wheat bran in yogurt as a fermented food model

- Effects of wheat bran addition on the growth/survival of lactic acid bacteria

5. To characterize phenolic composition of wheat bran by HPLC-PDA 
- Alkaline hydrolysis of phenolics and characterization

This thesis is publication based, and each chapter covers the manuscripts already published or soon to be published.

Chapter 1 provides background information on wheat, as well as on the extraction, quantification, and determination of ARs, and on the bioactivity, stability, and prebiotic activity of wheat bran extracts.

Chapter 2 evaluates the effects of cultivar and region on the composition of ARs in wheat grown in Ontario $(\mathrm{ON})$, using GC-MS. The effects of region and cultivar on the ARs in WB are closely examined and the results can be used for screening and breeding purposes.

Chapter 3 determines whether ARs in bread are stable during baking. This chapter gives detailed information about how different amounts of AR affect the homologue composition during baking.

Chapter 4 compares the traditional AR extraction method with the $\mathrm{SC}-\mathrm{CO}_{2}$ extraction method, the characterization of ARs by HPLC, as well as comparing different solvent systems in measuring antioxidant activity of WB extracts.

Chapter 5 investigates the capability and efficiency of WB soluble dietary fibre to enhance bacterial survival and growth in yogurt as well as to measure the bioactivity of the polysaccharides.

Finally, in Chapter 6, the overall conclusion is presented along with the contributions this thesis has made to current knowledge. It summurizes all findings and provides direction for future studies. 
Outcomes of this research might lead to practical applications of wheat bran ARs and soluble dietary fiber in food, and nutraceutical applications for Agri-Food companies. Furthermore, enhanced WB utilization will reduce Agri-Food by product/leftovers and thus improve environmental sustainability. 


\subsection{Contribution of the authors to the manuscript based chapters}

Chapters 2-5 are manuscript-based, and have been either presented in their edited form, or are soon to be published. Selected results from these chapters were also used to prepare conference presentations. The titles of the manuscripts and conference presentations are specified in the connecting statements for each chapter.

The contributions of the authors to the research presented in this thesis are as follows:

- Mrs. Aynur Gunenc, in her capacity as the author of this thesis and first author of the manuscripts and the conference presentations enumerated in the connecting statements to each manuscript-based chapter, conducted the reviews of literature, designed the experiments, the practical experimental work in the laboratory, and interpretation of results, the writing of this thesis, and the preparation of the manuscripts for publication.

- Dr. Farah Hosseinian, in her capacity as $\mathrm{PhD}$ Thesis supervisor, provided her technical expertise, guided the author of this thesis throughout all the stages of planning and experimental work, analyzing and interpreting the results, writing, and correcting this thesis, and preparing the manuscripts for publications.

- Dr. Mehri Hadinezhad, the Research Associate of Dr. Hosseinian Lab, provided technical expertise during the revision of the manuscript and submission process.

- Dr. Koushik Seetharaman from Guelph University provided the wheat bran samples.

- Dr. Paul Mayer from the University of Ottawa granted the use of his GC-MS instrument for analysis and characterization of the AR extracts. 
- Dr. Lily Tamburic-Ilincic from Guelph University provided wheat bran samples grown in different location of Ontario and years for screening purposes.

- David Fairbanks from Algonquin College aided in making the bread used experimentally as reported in Chapter 3, and allowed the use of their culinary facilities during the research concerning the stability of ARs during baking. 


\subsection{Connecting Statement to Chapter 2}

In Chapter 1, the economic importance of the wheat crop, the composition of WB, the health benefits of WB, and extraction methods, and the methods to analyze ARs content have been presented and discussed.

In Chapter 2, the contributions that the cultivar and region have on the ARs content and antioxidant activity of the wheat grown in Ontario, Canada are investigated.

The work presented in Chapter 2 has been used to prepare one conference presentation (poster) and one publication, as follows:

- Gunenc, A. Tamburic-Ilincic, L., and Hosseinian, F. New Approach for food safety assessment of Ontario wheat, OMAFRA-the 9th Annual Food Safety Research Forum, Guelph, ON, Canada, May $5^{\text {th }}, 2011$.

- Gunenc, A. Tamburic-Ilincic, L., and Hosseinian, F. New Approach for food safety assessment of Ontario wheat, OCCI (Ottawa-Carleton Chemical Institute) Poster Day, University of Ottawa, Ottawa, ON, Canada, 2011.

- Gunenc, A., Hadinezhad, M., Tamburic-Ilincic, L., Mayer, P. and Hosseinian, F. Effects of region and cultivar on alkylresorcinols content and composition in wheat bran and their antioxidant activity, Journal of Cereal Science, 2013, 57: 405-410. 


\section{Chapter 2 Effects of region and cultivar on alkylresorcinols content and composition in wheat bran and their antioxidant activity}

\subsection{Abstract}

This study evaluated the effects of cultivar and region on the composition of alkylresorcinols (ARs) of 24 wheat bran samples from 6 cultivars grown in four locations (Bath, Nairn, Palmerstone and Ridgetown) in Ontario, Canada, using gas chromatography/mass spectrometry (GC-MS). Total phenolic content (TPC) of wheat bran extracts was determined by Folin-Ciocalteau method and the antioxidant activity of wheat bran extracts was measured by 2,2-diphenyl-1-picryhydrazyl radical (DPPH) scavenging activity and oxygen radical absorbance capacity (ORAC) assays. The highest AR contents $(\mu \mathrm{g} / \mathrm{g})$ were found in cultivars Emmit (1522), Harvard (1305), Warthog (1170), and Superior (853) grown in Ridgetown. The relative percentages of saturated and unsaturated ARs were $89 \%$ and $11 \%$, respectively. Total AR content and composition, TPC, and the antioxidant activity of wheat bran extracts were significantly affected $(P<0.05)$ by location and cultivar. TPC, \% DPPH, and ORAC values for different wheat bran sample extracts ranged from 3 - $58 \mathrm{mg} \mathrm{FAE} / \mathrm{g}, 5$ - 68 (\%), and 6 - 94 $(\mu \mathrm{mol} \mathrm{TE} / \mathrm{g})$, respectively. This work provides a detailed examination of region and cultivar effects on the potential of ARs in wheat bran and results from which can be used for screening and breeding purposes. 


\subsection{Introduction}

\subsubsection{Climatic conditions affect wheat production}

Wheat, maize and rice are the three most important cereal crops, representing approximately 2 billion tons of the total global production. Amongst these three crops, wheat is the most widely grown and consumed cereal by humans. ${ }^{125}$ Wheat production needs suitable growing season temperatures and precipitation to obtain commercial yields.

Table 2.1 shows the ten year average Canadian wheat production and yield $(1,000$ tonnes) by province. The majority of Canadian wheat is produced in Saskatchewan (46\% of total production), Alberta (30\%), Manitoba (14\%), Ontario (9\%), Quebec (1\%) and the Atlantic region produces less than $1 \%$ of total wheat production.

The climatic range for wheat production along with the variability in genotypes for each wheat class means that yields and quality vary significantly by location. Also the climatic values represent long term mean conditions and there is considerable variation from year to year. ${ }^{126}$ 
Table 2-1 Average 1999-2008 Canadian wheat production and yield by province ${ }^{\mathbf{1 2 7}}$

\begin{tabular}{lrrrrrr}
\hline & Wheat & Production & ('000 tonnes) & Wheat & Yield & (Kg/ha) \\
\cline { 2 - 6 } Province & $\begin{array}{l}\text { Spring } \\
\text { Wheat }\end{array}$ & Durum & Winter Wheat & $\begin{array}{c}\text { Spring } \\
\text { Wheat }\end{array}$ & Durum & $\begin{array}{c}\text { Winter } \\
\text { Wheat }\end{array}$ \\
\hline \multirow{2}{*}{ British Columbia } & 59 & & & & & \\
Alberta & 6,064 & 841 & 145 & 2,740 & 2,410 & 3,000 \\
Saskatchewan & 7,674 & 3613 & 227 & 2,060 & 2,020 & 2,560 \\
Maniotoba & 3,184 & 44 & 421 & 2,730 & 2,314 & 3,690 \\
Ontario & 183 & & 1,636 & 3,310 & & 5,020 \\
Quebec & 134 & & 6 & 3,090 & & 2,990 \\
New Brunswick & 9 & & 2 & 3,070 & & 3,370 \\
Nova Scotia & 3 & & 8 & 3,020 & & 3,930 \\
Prince Edward & & & & & & \\
Island & 28 & & 7 & 2,990 & & 3,020 \\
Canada & 17,338 & 4,485 & 2,452 & 2,370 & 2,080 & 4,310 \\
\hline
\end{tabular}

\subsubsection{Wheat bran-phytochemicals affected by environmental factors}

Phytochemicals present in grains have the potential to reduce human diseases such as cardiovascular diseases ${ }^{128}$, diabetes ${ }^{129}$ and cancer. ${ }^{130}$ Some of the mentioned health benefits may be attributed to antioxidant activity of phenolic compounds such as ferulic acid, other polyphenols (lignans, anthocyanins and alkylresorcinols (ARs)), caretonoids and vitamin $\mathrm{E}^{30}$

Wheat has significant levels of antioxidants, and among these phenolic compounds may elicit the highest health benefit. They exist in free, bound and soluble conjugated forms. The most dominant free phenolic compounds are ferulic, $p$-coumaric and vanillic acids. They are found with other phenolics including caffeic, chorogenic, gentisic, syringic, and $p$-hydroxybenzoic acids. ${ }^{131}$ Previous studies have shown that bioactive 
compounds (tocopherols, sterols, ARs, folates, phenolic acids, and fiber components) can be affected by different environmental factors. ${ }^{52,125,132}$ The interactions between wheat genotype and the environment in which wheat is grown, and possibly genotypeenvironment interactions can likely strongly influence the levels of grain antioxidants. ${ }^{132}$ Shewry et al. (2010) showed significant correlations between the contents of bioactive components (ARs, sterols, tocols, folates, phenolic acids and fiber compounds) and environmental factors (precipitation and temperature) differing in amount between grain samples grown in different years or different sites. ${ }^{125}$

Quantification of phytochemicals in whole grains and their products is crucial for marketing purposes. Unfortunately, studies mainly select for new cultivars having high yield. In some countries such as Canada and Australia, end use quality is an another purpose for breeding. ${ }^{133}$

\subsubsection{Alkylresorcinols in wheat bran}

ARs are phenolic lipids, also called resorcinolic lipids, predominantly present in the bran fraction of rye grains, closely followed by triticale and wheat. ${ }^{35,134}$ ARs are located in the intermediate layers of pericarp and testa in the grain. ${ }^{45}$ The structure of ARs is 1, 3-dihydroxy-5-alkylbenzene derivatives with odd-numbered, mostly saturated hydrocarbon side chains in the range of 15-25 carbon atoms. Also, ARs have been suggested as markers for whole grain, rye products and a biomarker for human intake of whole grain wheat and rye even though the total ARs in wheat or rye differs in cereal species, the relative homologue composition of ARs remains almost constant within the species. $^{49}$

ARs affect physicochemical properties of biological membranes due to their 
amphiphilic character. They have their antibacterial and antifungal activity. ${ }^{30}$ They have potential to modulate the activity of some enzymes, and improve membrane phospholipid bilayer properties. ${ }^{135}$ For instance, long chain ARs mixtures have been reported to prevent the peroxidation of fatty acids and phospholipids in liposomal membranes as well as autoxidative processes in triglycerides and fatty acids. ${ }^{136}$

ARs in cereal grains and cereal grain products have been quantified using gas chromatography (GC) ${ }^{137}$ and high performance liquid chromatography (HPLC)Coularray-Based Electrochemical Detection. ${ }^{134}$ Also, AR metabolites in human urine have been determined by gas chromatography/mass spectrometry (GC-MS). ${ }^{138}$

\subsubsection{Objectives}

The primary objective of this study was to determine the effects of cultivar and region on AR content and composition of 24 wheat bran samples from 6 wheat cultivars grown in 4 different regions in Ontario using GC-MS. Also, another objective was to evaluate the effect of AR content and total phenolic content (TPC) on the antioxidant activity of wheat bran extracts by measuring oxygen radical absorbance capacity (ORAC) and 2, 2-diphenyl-1-picryhydrazyl (DPPH) radical scavenging activity.

\subsection{Materials and methods}

\subsubsection{Samples}

The wheat bran samples were provided by the Plant Agriculture Department at Guelph University, Ridgetown, Ontario (ON), Canada. The samples were from six winter wheat cultivars including three hard red wheats (AC Morley, Harvard and Warthog) and three soft red wheat (Emmit, Superior and FT Wonder). The samples were grown in 4 
different regions of Ontario in 2008; Bath, Nairn, Palmerstone and Rigdetown. Each region is illustrated in the map below of Ontario (Figure 2.1). The average precipitation, maximum and minimum temperatures from May $1^{\text {st }}$ to June $31^{\text {st }}$ in 2008 in the aforementioned regions were $265 \mathrm{~mm}, 21.5^{\circ} \mathrm{C}$ and $9.5^{\circ} \mathrm{C}$ for Bath; $340 \mathrm{~mm}, 21.0^{\circ} \mathrm{C}$ and $10.1{ }^{\circ} \mathrm{C}$ for Nairn; $394 \mathrm{~mm}, 19.1{ }^{\circ} \mathrm{C}$ and $8.5^{\circ} \mathrm{C}$ for Palmerstone; $340 \mathrm{~mm}, 20.9{ }^{\circ} \mathrm{C}$ and $9.9^{\circ} \mathrm{C}$ for Ridgetown, respectively (Table 2.2 ).

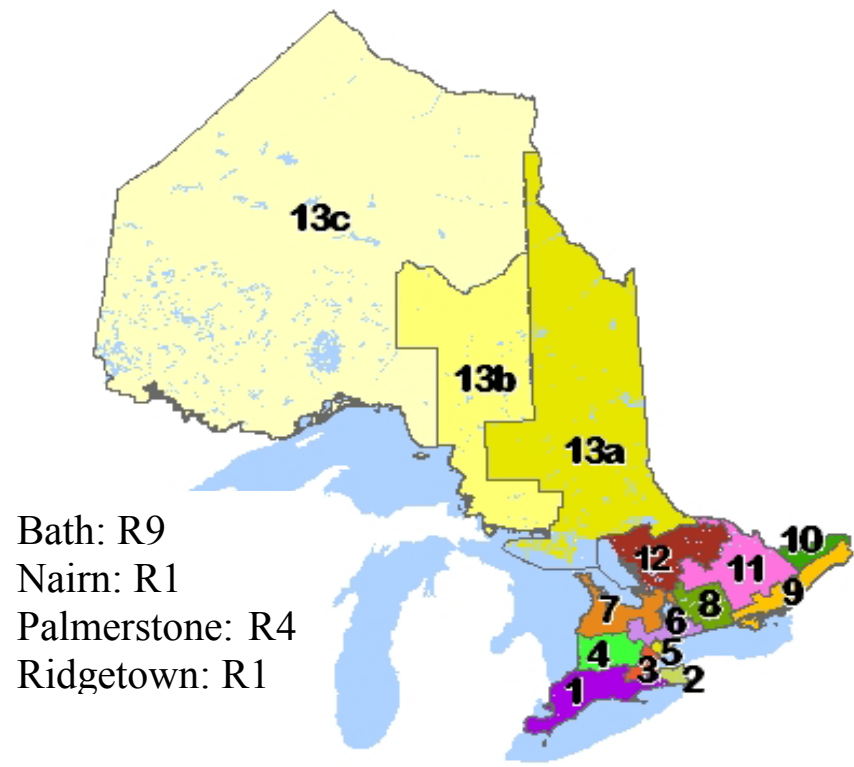

Figure 2-1 The map of Ontario regions, adopted from Ontario Ministry of tourism, culture and sport ${ }^{139}$

Table 2-2 The average precipitation $(\mathrm{mm})$, and temperatures $\left({ }^{\circ} \mathrm{C}\right)$ for each region

\begin{tabular}{lcccc}
\hline Region & Precipitation & T-min & T-max & $\Delta \mathrm{T}$ \\
\hline Bath & 265 & 9.5 & 21.5 & 12.0 \\
Nairn & 340 & 10.1 & 21.0 & 10.9 \\
Palmerstone & 394 & 8.5 & 19.1 & 10.6 \\
Ridgetown & 340 & 9.9 & 20.9 & 11.0 \\
\hline
\end{tabular}


Fine wheat (hard and soft) bran was prepared by abrading outer layers of grain in a Satake TM 05 laboratory scale pearler (Satake Co, Japan) to remove 10\% (by weight) of bran. Prior to extraction, the bran was ground to the recommended $2 \mathrm{~mm}$ size ${ }^{140}$ using a Thomas Wiley Mill (model ED-5, Arthur H. Thomas Co., Philadelphia, Pennsylvania, USA). Samples (24 wheat bran) were stored in sealed plastic bags and kept in the freezer $\left(-20^{\circ} \mathrm{C}\right)$ prior to analysis.

\subsubsection{Materials}

This section contains all detailed information about materials used not only for Chapter 2 but also for the following Chapters including 3, 4 and 5 .

N-O-bis-(trimethylsilyl)-tri-fluoroacetamide with $1 \%$ trimethylchlorosilane was purchased from VWR International (Ottawa, Canada) for derivatization of samples before $\mathrm{GC}$ analysis. Solvents, acetone, ethanol, $\mathrm{HCl}, 1$-propanol, and ethyl acetate were analytical grade and purchased from Caledon Laboratories LTC (Georgetown, ON, Canada). The AR standards (C15:0, C17:0, C19:0. C21:0, C23:0 and C25:0) in addition to $\mathrm{C} 22: 0$ (Internal standard) were over 98\% pure and purchased from ReseaChem $\mathrm{GmbH}$ (Burgdorf, Switzerland). Mono-and dibasic potassium phosphate, fluorescein, trolox (6hydroxy-2,5,7,8-tetramethylchroman-2-carboxylic acid), rutin, 2, 2'-azobis (2methylpropionamidine) dihydrochloride (AAPH), Folin-Ciocalteau reagent, $\alpha-$ tocopherol, and 2, 2-diphenyl-1-picryhydrazyl radical (DPPH), $\mathrm{NaOH}$ and phenolphthalein indicators were over 98\% pure and obtained from Sigma (Oakville, ON, Canada). Ferulic acid standard (99\% purity) was purchased from Fluka Analytical

(Sigma) (Oakville, ON, Canada). Sodium carbonate was obtained from Church and Dwight Canada Corp. (Mississauga, ON, Canada). 
The enzymes, $\alpha$-amylase from Bacillus licheniformis (Type XII-A, saline solution $\geq 500 \mathrm{U} / \mathrm{mg}$ protein, EC 232-752-2) and protease from Bacillus licheniform (saline solution $\geq 2.4$ U/g protein, EC 232-560-9) were purchased from Sigma-Aldrich (St. Louis, Missouri, USA). The starter cultures of Lactobacillus delbrueckii ssp. bulgaricus (B-548; USDA) and Streptococcus salivarius ssp. thermophilus (14485; ATCC) were employed. The probiotics used were Lactobacillus acidophilus (B-4495, USDA) and Bifidobacterium lactis (41405, USDA), Man Rogosa Sharpe (MRS) broth liquid and MRS agar media were purchased from Oxoid Ltd. (Basingstoke, United Kingdom).

The phenolic acid standards (over 97\% purity); gallic, proto-catechic, $\mathrm{p}-\mathrm{OH}-$ benzoic, chlorogenic, caffeic, vanillic, syringic, p-coumaric, sinapic, ferulic, o-coumaric, the flavanoid standards; pyrogallol, catechin, epicatechin, rutin, quercetin-3-betaglucoside, epicatechin gallate, myricetin, quercetin, apigenin and kaempherol were purchased from Sigma-Aldrich (St. Louis, Missouri, USA)

\subsubsection{Extraction of alkylresorcinols (ARs)}

ARs in wheat bran samples $(1 \mathrm{~g})$ were extracted with acetone in a 1:40 (w/v) ratio for $24 \mathrm{~h}$, by continuous stirring (Stirrer-VWR, Corning®, VMS-C4) at room temperature $^{140}$ and filtered using Whatman double filter paper (number 1). The precipitate was discarded and the acetone was evaporated to dryness from the supernatant using a Rotavapor (Buchi-Brinkman, R110, Switzerland). The extract was stored in the dark at $-20^{\circ} \mathrm{C}$ until further analysis of $\mathrm{TPC}, \mathrm{DPPH}, \mathrm{ORAC}$ and GC-MS. All measurements were conducted in triplicate. 


\subsubsection{GC-MS analysis}

GC-MS analysis was performed according to the method of Athukorala et al. ${ }^{35}$ Extracts $(200 \mu \mathrm{l})$ were spiked with $200 \mu \mathrm{l}$ of internal standard AR C22:0 $(0.5 \mathrm{mg} / \mathrm{mL})$. The solvent was evaporated under nitrogen, and were derivatized with trimethylsilyl (TMS) using $200 \mu \mathrm{L}$ of bis-(trimethylsilyl)-trifluoroacetamide containing $1 \%$ trimetylchlorosilane (TMSC) and $100 \mu 1$ of ethyl acetate. The reaction mixture was heated at $75^{\circ} \mathrm{C}$ for $30 \mathrm{~min}$ and excess reagent was then evaporated under nitrogen and redissolved in ethyl acetate for GC-MS analysis. The GC-MS analysis was conducted with a Hewlett Packard HP 6890 Plus (GC) and Model G1540A equipped with a network mass selective detector. A DB-17HT high-temperature capillary column was used (30 m x $0.25 \mathrm{~mm}$ I.D., $0.1 \mu \mathrm{m}$ film thicknesses, J \& W Scientific, Folsom, California). Helium was used as the carrier gas with a flow rate of $1.3-\mathrm{mL} / \mathrm{min}$ and split injection by $10: 1$ ratio. The initial oven temperature of $150^{\circ} \mathrm{C}$ was held for $2 \mathrm{~min}$ and the temperature was increased by $10{ }^{\circ} \mathrm{C} / \mathrm{min}$ to $320^{\circ} \mathrm{C}$. Upon reaching $320^{\circ} \mathrm{C}$, it was held for $7 \mathrm{~min}$ and the temperature was increased to $325^{\circ} \mathrm{C}$ for $1 \mathrm{~min}$. A Hewlett Packard HP 5973 (MS) was operated in the electron ionization (EI) mode at $70 \mathrm{eV}$, a source temperature of $280^{\circ} \mathrm{C}$ and temperature of $150^{\circ} \mathrm{C}$, in the scan range of $\mathrm{m} / z 35$ to 350 . Data were collected by ChemStation software (Shawnee, KS, USA). Identification of components was done by comparing their spectral data with ARs-standards (C15:0 to C25:0). The MS was used to confirm the presence of AR homologues by their molecular ion peaks at m/z 320 (C15:0), 348 (C17:0), 376 (C19:0), 404 (C21:0), 432 (C23:0) and 460 (C25:0). Data were compared against the NIST (v.02) and Wiley (v.138) libraries (Palisade Corp., Newfield, New York). ${ }^{35}$ 


\subsubsection{Measuring total phenolic content (TPC) of wheat bran extracts}

TPC was determined by using modified procedures of the Folin-Ciocalteu method

of Gao et al. ${ }^{141}$ Folin-Ciocalteu reagent was diluted 10 times with deionized water. The extract $(200 \mu \mathrm{L})$ was added to of freshly diluted Folin-Ciocalteu reagent $(1.9 \mathrm{~mL})$. Then $1.9 \mathrm{~mL}$ of sodium carbonate solution $(60 \mathrm{~g} / \mathrm{L})$ was added to the mixture. It was left for 2 $h$ incubation at ambient temperature and the absorbance of the mixture was read at 725 $\mathrm{nm}$ against a blank of distilled water by using the Cary 50Bio UV-Visible Spectrophotometer (Varian Inc., Australia). Ferulic acid was used as standard. ${ }^{142}$

\subsubsection{Measuring antioxidant activity by DPPH scavenging activity assay of wheat bran extracts}

DPPH scavenging activity was performed according to a modified method of Brand-Williams et al. ${ }^{142} 200 \mu \mathrm{L}$ of extracts were reacted with $3.8 \mathrm{~mL}$ of DPPH solution (60 $\mu \mathrm{m}$ DPPH solution freshly prepared in 95\% ethanol solution). The absorbance (A) values of the mixture at $515 \mathrm{~nm}$ were read against a blank of pure $95 \%$ ethanol at 0 and 30 minutes. Moreover, DPPH assay was performed on each standard AR homologue and a mixture of standard AR homologues at equal concentrations ( $2 \mathrm{mg} / \mathrm{ml}$ ethanol solution). Antioxidant activity was calculated as percent discoloration as shown in the following equation. $^{142}$

$$
\% D P P H=\left[1-\left(\frac{A_{\text {Sample,t }=30 \min }}{A_{\text {Controlt } t=o \text { min }}}\right)\right] \times 100
$$




\subsubsection{Oxygen radical absorbance capacity (ORAC) assay of wheat bran extracts}

The ORAC of each wheat bran extract as well as AR standards was measured according to the procedure described by Ou et al. ${ }^{143}$ The ORAC assay was carried out on a fluorometric micro plate reader (FLx800 ${ }^{\mathrm{TM}}$ Multi-Detection Microplate Reader with Gen5 ${ }^{\mathrm{TM}}$ software, BioTek Instruments, Ottawa, Canada). Peroxyl radicals were generated by AAPH, and fluorescence microplate reader was used at an excitation wavelength of $485 \mathrm{~nm}$ and an emission wavelength of $525 \mathrm{~nm}$. Trolox was used as standard $(100,50$, $25,12.5,6.25 \mu \mathrm{M})$ and rutin was used as control $(10$ and $20 \mu \mathrm{M})$. Proper dilutions of bran extracts were made with ORAC buffer (potassium phosphate buffer, $\mathrm{pH}$ 7.4). For each ORAC run, a micro plate was prepared containing $20 \mu \mathrm{L}$ of Trolox standards, Rutin control, and sample dilutions, as well as $120 \mu \mathrm{L}$ of fluorescein (FL) solution. All ORAC analyses were performed at $37^{\circ} \mathrm{C}$ with a 20 min incubation and 60 min run time. After the incubation, $60 \mu \mathrm{L}$ of $153 \mathrm{mM}$ AAPH was added to each well for a final volume of $200 \mu \mathrm{L}$. The results were calculated using the differences of areas under the FL decay curves between the blank and a sample and were expressed as micromole Trolox Equivalents per gram of sample $(\mu \mathrm{mol} \mathrm{TE} / \mathrm{g}){ }^{143}$

\subsubsection{Statistical analyses}

All statistical analyses were performed using a two-way-analysis of variance (ANOVA) (SAS version 9.2, SAS Institute Inc., Cary, NC, USA). Significant treatments were further analyzed with the Duncan's multiple range test $(P<0.05)$. Significant correlations $(P<0.05)$ between ARs content and other measured parameters were performed using Microsoft Excel (Version 2010). 


\subsection{Results and Discussion}

\subsubsection{ARs analysis of wheat bran using GC-MS}

\subsubsection{Total ARs content and related composition}

The total AR contents $(\mu \mathrm{g} / \mathrm{g})$ as well as the relative \% saturated and unsaturated AR homologue contents of wheat bran samples from 6 cultivars grown in 4 different regions of $\mathrm{ON}$ in 2008 are shown in Table 2.3. Also, the GC-MS chromatograms for AR-standards and AR extract from the Emmit cultivar grown in Ridgetown is shown in Figure.2.2.

The saturated ARs were confirmed by their molecular ion as follows, at m/z 464 (C15:0), 492 (C17:0), 520 (C19:0), 548 (C21:0), 576 (C23:0), 604 (C25:0) and the base peak at m/z 268 for all ARs. The peaks with this characteristic $\mathrm{m} / \mathrm{z}$ value were considered resorcinolic derivatives. The same observations were reported in literature. ${ }^{34,35}$ The unsaturated peaks were assigned according to the MS data obtained from each GC-MS run and compared with literature data. ${ }^{34}$ Among 24 wheat bran samples, ARs were found in 23 samples (Table 2.3). The cultivar Emmit had the highest amount of total ARs (1522 $\mu \mathrm{g} / \mathrm{g}$ wheat bran sample) compared to all other cultivars. Athukorala et al.(2010), also reported $680 \mu \mathrm{g} / \mathrm{g}$ as total ARs contents in wheat bran sample from a commercial

source. ${ }^{35}$ The cultivar AC Morley grown in Palmerstone region was found to be free of ARs. It is not clear why this cultivar did not show any detectable quantity of ARs. More studies on another sample from the same cultivar and region are needed to confirm this result.

ARs in wheat are mostly present in the outer layers of bran. ${ }^{90}$ Landberg et al. (2007) analyzed total ARs in different wheat grain fractions and reported 576, 2923 and 
$1024 \mu \mathrm{g} / \mathrm{g}$ of total ARs in ethyl acetate extracts of whole grain wheat, wheat bran and wheat aleurone, respectively. ${ }^{68}$ Therefore, the bran removal technique used is an important factor in determining the total AR content. During grain milling, contamination of bran with refined flour could result in much lower ARs detection in wheat bran.

Although the total ARs content shown as in Table 2.3 was different for each cultivar, the relative saturated: unsaturated ratio of ARs remained close to 89:11and our findings were in agreement into the values reported in literature. ${ }^{137}$ 
Table 2-3 Total ARs content in $\mu \mathrm{g} / \mathrm{g}$, and related \% saturated (Sat), unsaturated (Unsat) and homologue

\begin{tabular}{|c|c|c|c|c|c|c|c|}
\hline Region & Composition & AC Morley & Emmit & FT Wonder & Harvard & Superior & Warthog \\
\hline \multirow[t]{8}{*}{ Bath } & Total- ARs & $338^{\mathrm{jk}}$ & $138^{\mathrm{Im}}$ & $137^{\operatorname{lm}}$ & $367^{\mathrm{jk}}$ & $175^{1}$ & $216^{1}$ \\
\hline & Sat.ARs & $88^{\mathrm{h}}$ & $100^{\mathrm{a}}$ & $81^{\mathrm{j}}$ & $90^{\text {defg }}$ & $84^{\mathrm{i}}$ & $88^{h}$ \\
\hline & Unsat. ARs & $12^{c}$ & nd & $19^{\mathrm{a}}$ & $10^{\mathrm{def}}$ & $16^{\mathrm{b}}$ & $12^{\mathrm{c}}$ \\
\hline & $\mathrm{C} 17: 0$ & 4 & 3 & nd & 4 & 3 & 3 \\
\hline & C19:0 & 31 & 24 & 28 & 31 & 22 & 25 \\
\hline & $\mathrm{C} 21: 0$ & 42 & 51 & 53 & 43 & 41 & 41 \\
\hline & $\mathrm{C} 23: 0$ & 11 & 14 & nd & 8 & 12 & 13 \\
\hline & $\mathrm{C} 25: 0$ & nd & 8 & nd & 4 & 6 & 6 \\
\hline \multirow[t]{8}{*}{ Nairn } & Total- ARs & $458^{\mathrm{ij}}$ & $664^{\mathrm{gf}}$ & $509^{\mathrm{hi}}$ & $60^{\text {gh }}$ & $801^{\mathrm{de}}$ & $259^{\mathrm{kl}}$ \\
\hline & Sat.ARs & $90^{\text {fgh }}$ & $91^{\mathrm{cd}}$ & $92^{\mathrm{cd}}$ & $89^{\text {fgh }}$ & $89^{\text {fgh }}$ & $88^{\text {fgh }}$ \\
\hline & Unsat. ARs & $10^{\text {cde }}$ & $9^{\text {efg }}$ & $8^{g}$ & $11^{\mathrm{c}}$ & $11^{\text {cde }}$ & $12^{\mathrm{c}}$ \\
\hline & $\mathrm{C} 17: 0$ & 2 & 2 & 2 & 2 & 2 & 2 \\
\hline & C19:0 & 25 & 22 & 23 & 27 & 19 & 25 \\
\hline & $\mathrm{C} 21: 0$ & 47 & 51 & 54 & 46 & 51 & 42 \\
\hline & $\mathrm{C} 23: 0$ & 11 & 11 & 8 & 9 & 11 & 13 \\
\hline & $\mathrm{C} 25: 0$ & 5 & 5 & 5 & 5 & 6 & 6 \\
\hline \multirow[t]{8}{*}{ Palmerstone } & Total- ARs & $\mathrm{nd}^{\mathrm{b}}$ & $779^{\mathrm{def}}$ & $845^{\mathrm{de}}$ & $21^{\mathrm{m}}$ & $137^{\mathrm{lm}}$ & $363^{\mathrm{jk}}$ \\
\hline & Sat.ARs & nd & $93^{c}$ & $92^{\mathrm{cd}}$ & $100^{\mathrm{a}}$ & $100^{\mathrm{a}}$ & $90^{\text {def }}$ \\
\hline & Unsat. ARs & nd & $7^{g}$ & $8^{\mathrm{gf}}$ & nd & nd & $10^{\mathrm{def}}$ \\
\hline & $\mathrm{C} 17: 0$ & nd & 3 & 2 & nd & nd & 3 \\
\hline & C19:0 & nd & 28 & 16 & 43 & 24 & 28 \\
\hline & C21:0 & nd & 45 & 38 & 57 & 76 & 45 \\
\hline & C23:0 & nd & 11 & 33 & nd & nd & 12 \\
\hline & $\mathrm{C} 25: 0$ & nd & 6 & 3 & nd & nd & 22 \\
\hline \multirow[t]{8}{*}{ Ridgetown } & Total- ARs & $366^{\mathrm{jk*}}$ & $1522^{\mathrm{a}}$ & $725^{\text {ef }}$ & $1306^{b}$ & $853^{d}$ & $1170^{c}$ \\
\hline & Sat.ARs & $83^{\mathrm{ij}}$ & $92^{\mathrm{cd}}$ & $97^{b}$ & $91^{\text {cde }}$ & $90^{\text {efgh }}$ & $90^{\mathrm{def}}$ \\
\hline & Unsat. ARs & $17^{\mathrm{ab}}$ & $8^{\mathrm{gf}}$ & $3^{h}$ & $9^{\mathrm{fg}}$ & $10^{\text {cde }}$ & $10^{\mathrm{def}}$ \\
\hline & $\mathrm{C} 17: 0$ & 3 & 3 & 3 & 2 & 2 & 3 \\
\hline & C19:0 & 24 & 26 & 30 & 30 & 19 & 26 \\
\hline & $\mathrm{C} 21: 0$ & 41 & 49 & 48 & 48 & 50 & 46 \\
\hline & C23:0 & 11 & 10 & 11 & 8 & 13 & 10 \\
\hline & $\mathrm{C} 25: 0$ & 4 & 4 & 5 & 3 & 6 & 5 \\
\hline
\end{tabular}

${ }^{a}$ Values are means of triplicates with different letters in rows for each characteristic are significantly different $(P<0.05)$ in Duncan's multiple range tests

${ }^{\mathrm{b}}$ nd $=$ not detected 

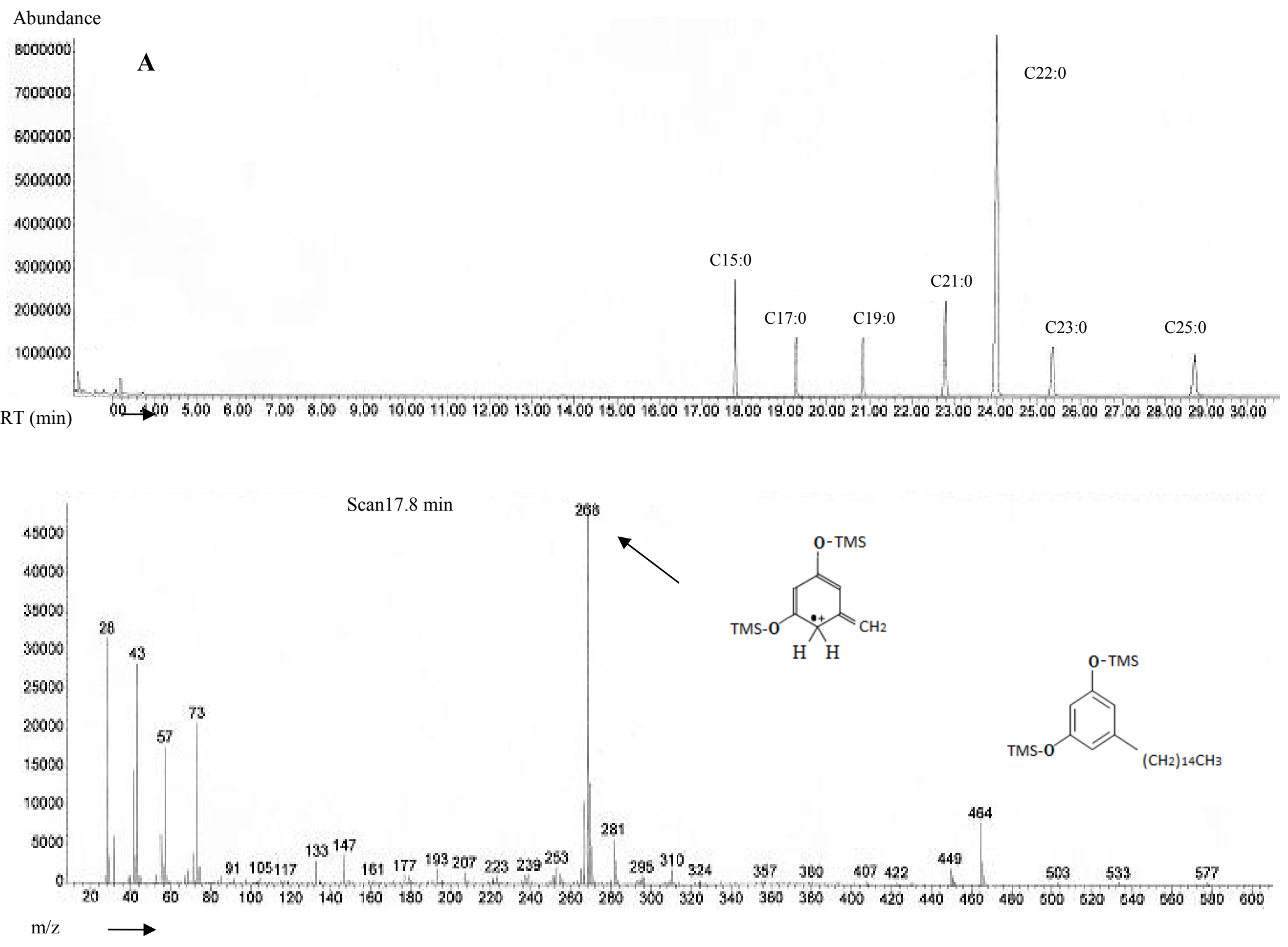


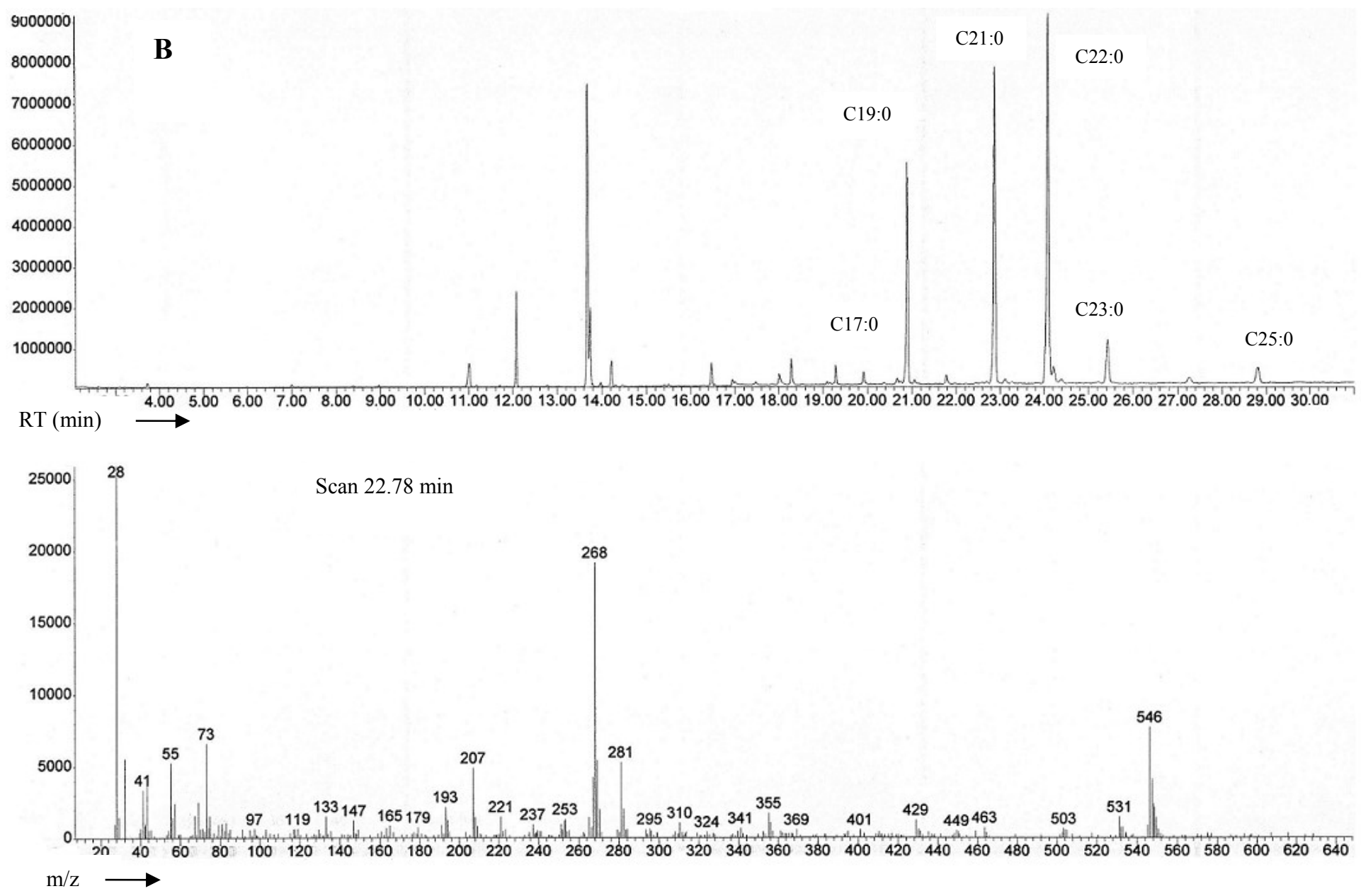

Figure 2-2 A) GC-MS of derivitized ARs standards (C15-C25) and the fragmentation pattern, B) GC-MS of cultivar Emmit grown in Ridgetown region. 


\subsubsection{Effects of region and cultivar}

Two-way ANOVA demonstrated that the region, cultivar and interaction of the two showed significant $(P<0.001)$ effects on all measured parameters (Table 2.4).

Average total AR contents for different wheat varieties were highest in Ridgetown and lowest in Bath regions. On the other hand, for each region, the cultivar Emmit had the highest overall total AR contents with AC Morley having the lowest. The ANOVA also revealed that for each cultivar; the lower the total ARs, the higher the relative \%unsaturated ARs. Genetic factors, climate, season, grain maturity, cereal type and soil conditions have been reported to affect ARs content. ${ }^{38}$

Overall, average of total AR contents $(607 \mu \mathrm{g} / \mathrm{g})$ for soft wheat (Emmit, Superior, and FT Wonder) was higher than than that of hard red wheat (488 $\mu \mathrm{g} / \mathrm{g}$; AC Morley, Harvard, and Warthog). This finding was in agreement with the study of Hengtrakul et al.(1990), where hard wheat had lower AR content than soft wheat. ${ }^{144}$ 
Table 2-4 Alkylresorcinols (ARs) analysis results for cultivar and region effects

\begin{tabular}{|c|c|c|c|c|c|c|c|c|c|c|c|c|}
\hline Cultivar $^{\mathrm{e}}$ & $\begin{array}{l}\text { Total } \\
\text { ARs }^{\mathrm{a}}\end{array}$ & Sat.ARs & Unsat.ARs & C17:0 & C19:0 & $\mathrm{C} 21: 0$ & $\mathrm{C} 23: 0$ & $\mathrm{C} 25: 0$ & $\mathrm{C} 17 / \mathrm{C} 21$ & $\mathrm{TPC}^{\mathrm{b}}$ & $\mathrm{DPPH}^{\mathrm{c}}$ & ORAC $^{d}$ \\
\hline Morley & $387^{\mathrm{e}}$ & $86^{\mathrm{e}}$ & $14^{\mathrm{a}}$ & $2.8^{\mathrm{a}}$ & $26.9^{b}$ & $43.2^{c}$ & $10.2^{b c}$ & $2.9^{\mathrm{c}}$ & $0.07^{\mathrm{a}}$ & $29^{d}$ & $41^{b c}$ & $28^{d}$ \\
\hline Emmit & $776^{\mathrm{a}}$ & $95^{\mathrm{a}}$ & $5^{e}$ & $2.5^{b}$ & $24.9^{b c}$ & $50.1^{b}$ & $11.7^{\text {ba }}$ & $5.8^{a b}$ & $0.05^{\mathrm{c}}$ & $36^{b}$ & $39^{\mathrm{c}}$ & $47^{\mathrm{a}}$ \\
\hline FT Wonder & $554^{\mathrm{cb}}$ & $91^{\mathrm{c}}$ & $9^{c}$ & $1.7^{\mathrm{c}}$ & $24.4^{b c}$ & $47.7^{b}$ & $13.4^{\mathrm{a}}$ & $3.8^{b c}$ & $0.03^{\mathrm{d}}$ & $26^{\mathrm{e}}$ & $48^{\mathrm{a}}$ & $48^{\mathrm{a}}$ \\
\hline Harvard & $574^{b}$ & $93^{b}$ & $7^{d}$ & $2.2^{\mathrm{ab}}$ & $34.9^{\mathrm{a}}$ & $44.9^{\mathrm{c}}$ & $7.9^{\mathrm{c}}$ & $3.1^{\mathrm{c}}$ & $0.05^{b}$ & $29^{d}$ & $44^{b}$ & $44^{\mathrm{ab}}$ \\
\hline Superior & $491^{\mathrm{d}}$ & $91^{\mathrm{c}}$ & $9^{c}$ & $1.7^{\mathrm{c}}$ & $21.3^{\mathrm{c}}$ & $54.7^{\mathrm{a}}$ & $9.1^{\mathrm{abc}}$ & $4.2^{\mathrm{bc}}$ & $0.03^{\mathrm{d}}$ & $32^{c}$ & $37^{\mathrm{cd}}$ & $40^{\mathrm{bc}}$ \\
\hline Warthog & $502^{\mathrm{cd}}$ & $89^{d}$ & $11^{b}$ & $2.6^{\mathrm{a}}$ & $26.2^{b}$ & $41.2^{\mathrm{c}}$ & $11.2^{\mathrm{ab}}$ & $7.8^{\mathrm{a}}$ & $0.06^{b}$ & $40^{\mathrm{a}}$ & $35^{\mathrm{d}}$ & $38^{c}$ \\
\hline \multicolumn{13}{|l|}{$\underline{\text { Region }}$} \\
\hline Bath & $229^{d}$ & $89^{b}$ & $11^{\mathrm{a}}$ & $2.6^{\mathrm{a}}$ & $26.9^{\mathrm{a}}$ & $45.7^{b}$ & $9.9^{\mathrm{a}}$ & $3.9^{\mathrm{a}}$ & $0.07^{\mathrm{a}}$ & $33^{b}$ & $21^{\mathrm{d}}$ & $26^{\mathrm{d}}$ \\
\hline Nairn & $549^{b}$ & $90^{b}$ & $10^{b}$ & $2.3^{b}$ & $23.7^{\mathrm{a}}$ & $48.3^{\mathrm{a}}$ & $10.8^{\mathrm{a}}$ & $4.9^{\mathrm{a}}$ & $0.04^{\mathrm{c}}$ & $34^{b}$ & $50^{b}$ & $38^{b}$ \\
\hline Palmerstone & $358^{c}$ & $92^{\mathrm{a}}$ & $8^{d}$ & $2.8^{\mathrm{c}}$ & $26.9^{\mathrm{a}}$ & $47.7^{\mathrm{c}}$ & $10.3^{\mathrm{a}}$ & $4.8^{\mathrm{a}}$ & $0.05^{b}$ & $19^{\mathrm{c}}$ & $37^{\mathrm{c}}$ & $31^{\mathrm{c}}$ \\
\hline Ridgetown & $990^{\mathrm{a}}$ & $91^{\mathrm{b}}$ & $9^{c}$ & $2.6^{\mathrm{a}}$ & $26.6^{\mathrm{a}}$ & $46.7^{\mathrm{ab}}$ & $10.3^{\mathrm{a}}$ & $4.8^{\mathrm{a}}$ & $0.05^{b}$ & $42^{a}$ & $54^{\mathrm{a}}$ & $68^{a}$ \\
\hline
\end{tabular}

${ }^{\mathrm{a}}$ Total ARs $=\mu \mathrm{g} / \mathrm{g}$ of sample, Sat. ARs $=\%$ Saturated ARs, Unsat ARs =\%Unsaturated ARs, all ARs homologue $(\mathrm{C} 17: 0-\mathrm{C} 25: 0)$ are in $(\%)$.

${ }^{\mathrm{b}} \mathrm{TPC}=$ Total Phenolic Content as mg Ferulic Acid Equivalent (FAE) /g

${ }^{\mathrm{c}} \mathrm{DPPH}=1$, 1-diphenyl-2-picrylhydrazyl as \%DPPH inhibition after $30 \mathrm{~min}$

${ }^{\mathrm{d}}$ ORAC $=$ Oxygen Radical Absorbance Capacity as TE $\mu$ mole/g (Trolox Equivalent)

${ }^{\mathrm{e}}$ Values are means of triplicates with different letters in columns are significantly different $(P<0.0001)$ in Duncan's multiple range tests 


\subsubsection{The relative \% of homologue composition}

All assignments of homologues C15:0-C25:0 were made by comparison of retention times of the respective reference standards. Also, each peak assignment was confirmed by fragmentation pattern in the MS data. A homologous series of saturated C17:0-C25:0 were detected in wheat bran extracts which was in agreement with literature reports. ${ }^{145}$ In all wheat bran samples, $\mathrm{C} 21: 0$ and $\mathrm{C} 19: 0$ homologues were the most abundant. Wheat bran was also reported to have some AR derivatives including ARs with unsaturated alkyl chains, keto groups, or a combination of keto groups and unsaturated alkyl chains. ${ }^{78}$ It has been suggested that these minor groups of AR derivatives exhibit various biological activities related to their amphiphilic characteristics.

Only 1\% of the AR homologue, C15:0 was detected in cultivar Emmit in both Ridgetown and Nairn regions, and cultivar Superior in Bath region (data not shown). The highest to lowest AR homologue order was $\mathrm{C} 21>\mathrm{C} 19>\mathrm{C} 23>\mathrm{C} 25>\mathrm{C} 17$ for all samples. These findings are in agreement with results reported in the works of Ross et al. and Knodler et al..$^{34,145}$ The relative homologue composition of ARs in wheat samples has been reported to be 3-6\% $\mathrm{C} 17: 0,29-42 \% \mathrm{C} 19: 0,46-55 \% \mathrm{C} 21: 0,4-12 \% \mathrm{C} 23: 0$, and $1-4 \%$ $\mathrm{C} 25: 0$. The ratio of $\mathrm{C} 17: 0$ to $\mathrm{C} 21: 0$ for durum wheat, common wheat, and rye is 0.01 , 0.1 , and 1.0, respectively. ${ }^{38}$ The ratio of $\mathrm{C} 17: 0 / \mathrm{C} 21: 0$ ranges from 0.03 to 0.07 and these ratios are suggested to be an index for determining the cereal grain source. The ratio of

$0.01,0.1$ and 1.0 was reported for durum wheat, common wheat and rye respectively. ${ }^{130}$ Our study demonstrated that these ratios could be used to differentiate common wheat cultivars. 


\subsubsection{TPC of wheat bran extracts}

The TPC of all wheat bran samples were analyzed by Folin-Ciocalteu method. Results were significantly different $(P<0.05)$ among wheat bran-acetone extracts (Table 2.5). Wheat bran from cultivar Emmit grown in Ridgetown had the highest TPC (58 mg FAE/g) and it was lowest for Harvard cultivar from Palmerston (5 mg FAE/g). This wide range reflects the TPC dependency on the wheat cultivars. In wheat, several major phenolic compounds are present including phenolic acids, ARs, flavonoids, and phenolic acid diacyl glycerols, phenolic aldehydes and ferulates. ${ }^{30}$ There was a small but significant correlation between total ARs and TPC of wheat brans $\left(R^{2}=0.20, P<0.05\right)$. 
Table 2-5 TPC (mg FAE /g), \% DPPH, and ORAC ( $\mu$ mol TE/g) values* of WB extracts

\begin{tabular}{|c|c|c|c|c|c|c|c|}
\hline Region & Analysis & AC Morley & Emmit & FT Wonder & Harvard & Superior & Warthog \\
\hline \multirow[t]{3}{*}{ Bath } & $\mathrm{TPC}^{\mathrm{a}}$ & $33^{\text {fged }}$ & $43^{b}$ & $13^{j}$ & $31^{\text {fgeh }}$ & $35^{\text {ced }}$ & $41^{\mathrm{cb}}$ \\
\hline & $\% \mathrm{DPPH}^{\mathrm{b}}$ & $43^{\mathrm{fg}}$ & $11^{\mathrm{ml}}$ & $15^{\mathrm{kl}}$ & $18^{\mathrm{jkl}}$ & $19^{\mathrm{jk} l}$ & $20^{\mathrm{jk}}$ \\
\hline & ORAC $^{\mathrm{c}}$ & $41^{\text {egf }}$ & $21^{\mathrm{i}}$ & $20^{\mathrm{i}}$ & $29^{\text {ihg }}$ & $20^{\mathrm{i}}$ & $25^{\text {ih }}$ \\
\hline \multirow[t]{3}{*}{ Nairn } & $\mathrm{TPC}$ & $41^{\mathrm{cb}}$ & $28^{\text {gih }}$ & $29^{\text {fgih }}$ & $38^{\mathrm{cbd}}$ & $30^{\text {fgeh }}$ & $38^{\mathrm{cbd}}$ \\
\hline & $\% \mathrm{DPPH}_{\mathrm{c}}$ & $68^{a}$ & $26^{\mathrm{ji}}$ & $60^{\text {bac }}$ & $63^{\text {ba }}$ & $52^{\mathrm{edc}}$ & $33^{\mathrm{hi}}$ \\
\hline & ORAC & $33^{\text {ihgf }}$ & $36^{\text {ehgf }}$ & $56^{d}$ & $33^{\text {ihgf }}$ & $47^{\mathrm{ed}}$ & $24^{\text {ih }}$ \\
\hline \multirow[t]{3}{*}{ Palmerstone } & ТPC & $3^{k}$ & $15^{\mathrm{j}}$ & $27^{\text {ih }}$ & $5^{\mathrm{k}}$ & $24^{\mathrm{i}}$ & $38^{\mathrm{cbd}}$ \\
\hline & \% DPPHc & $5^{\mathrm{m}}$ & $60^{\text {bac }}$ & $63^{\mathrm{ba}}$ & $43^{\mathrm{fg}}$ & $18^{\mathrm{kl}}$ & $36^{\mathrm{hg}}$ \\
\hline & ORAC & $6^{\mathrm{i}}$ & $45^{\text {edf }}$ & $68^{c}$ & $20^{\mathrm{i}}$ & $20^{\mathrm{i}}$ & $28^{\text {ihg }}$ \\
\hline \multirow[t]{3}{*}{ Ridgetown } & ТРC & $39^{\mathrm{cbd}}$ & $58^{\mathrm{a}}$ & $34^{\mathrm{fed}}$ & $43^{b}$ & $37^{\mathrm{cbd}}$ & $42^{b}$ \\
\hline & $\%$ DPPHc & $47^{\mathrm{fe} *}$ & $57^{\mathrm{bdc}}$ & $56^{\text {bedc }}$ & $52^{\text {edc }}$ & $61^{\text {bac }}$ & 50 fed \\
\hline & ORAC & $29^{\text {ihg }}$ & $85^{\text {ba }}$ & $48^{\text {ed }}$ & $94^{\mathrm{a}}$ & $74^{\mathrm{bc}}$ & $75^{\mathrm{bc}}$ \\
\hline
\end{tabular}

${ }^{\mathrm{a}} \mathrm{TPC}=$ Total Phenolic Content as mg Ferulic Acid Equivalent (FAE) $/ \mathrm{g}$

${ }^{\mathrm{b}}$ DPPH $=1$, 1-diphenyl-2-picrylhydrazyl as \%DPPH inhibition after $30 \mathrm{~min}$

${ }^{\mathrm{c}}$ ORAC $=$ Oxygen Radical Absorbance Capacity as $\mu$ mole TE (Trolox Equivalent) $/ \mathrm{g}$

${ }^{*}$ Values are means of triplicates with different letters in rows for each analysis are significantly different $(P<0.05)$ in Duncan's multiple range tests

Two-way ANOVA showed that region, cultivar and their interactions were significantly different $(P<0.001)$ in TPC (Table 2.5). Average TPC was highest for Ridgetown and lowest for Palmerstone regions. Differences among the cultivars at each location were also significant $(P<0.001)$; Warthog had the highest TPC with FT Wonder having the lowest.

It should be mentioned that the present study analyzed acetone extracts of all wheat bran samples for TPC, ORAC and DPPH assays. As reported by Winata and Lorenz, our preliminary experiments also demonstrated that acetone extracts were more selective for AR extractions in comparison with ethanol, ethyl acetate, and propanol. ${ }^{146}$ 
However, it co-extracts other compounds which affect the measured TPC and antioxidant activity. Liyana-Pathirana and Shahidi reported TPC of 67 and $59 \mathrm{mg} \mathrm{FAE} / \mathrm{g}$ in soft and hard wheat bran, respectively, using $80 \%$ ethanolic crude extracts. ${ }^{147}$

\subsubsection{Antioxidant activity of wheat bran extracts by DPPH scavenging activity assay}

The antioxidant activity of all wheat bran-acetone extracts was measured using a DPPH assay (Table 2.5). This assay measures the ability of antioxidants to reduce the DPPH radical which can be calculated by the percentage of discolorization; the higher this percentage, the stronger the antioxidant. ${ }^{114}$ There were significant differences $(P<$ 0.05 ) in \% DPPH-discoloration of all assayed samples. All \% DPPH inhibition results ranged between 5 and 68. It was interesting the same cultivar, AC Morley, from different regions, possessed the highest and lowest antioxidant potentials based on this assay. There was a small but significant correlation between total ARs of wheat bran samples and their \% DPPH $\left(R^{2}=0.36, P<0.05\right)$.

Two-way ANOVA showed that region, cultivar and their interactions, were significantly different $(P<0.001)$ for antioxidant activity (Table 2.5). Mpofu et al. (2006) also reported that antioxidant properties of wheat and wheat products are affected by genotype and environment. ${ }^{132}$ Wheat brans from Ridgetown and Bath regions had the highest and lowest antioxidant activity, respectively. The antioxidant activity of different cultivars as $\% \mathrm{DPPH}$-discoloration ranged from $35 \%$ (Warthog cultivar) to $48 \%$ (FT Wonder cultivar) and the antioxidant activity of different regions ranged from 21\% (Bath region) to $54 \%$ (Ridgetown region). These findings suggest that region has a greater effect than cultivar type on antioxidant activity. 
Overall, the \%DPPH inhibition of soft wheat (41\%) was slightly higher than that of hard wheat (40\%). Using a similar protocol and DPPH method, Liyana-Pathirana and Shahidi (2006) obtained \% DPPH inhibition values of 58\% for soft wheat and 55\% for hard wheat brans extracted with $80 \%$ ethanol.

Table 2.6 shows the antioxidant activity of individual AR homologue standards and their mixture. At a ratio of 4-5 $\mathrm{mol} \mathrm{AR} / \mathrm{mol} \mathrm{DPPH}$ the antioxidant activity ranged from 20 to $30 \%$ discoloration of DPPH. The highest observed antioxidant activity $(31.5 \pm$ $1.8 \%$ ) belonged to the mixture of AR standards with a ratio of $4.3 \mathrm{~mol} / \mathrm{mol} \mathrm{DPPH}$, indicating some synergistic effects of the mixture. Kamal-Eldin et al.(2000) also reported a weak hydrogen donation activity for 5-n-pentadecylresorcinol (C15:0); $20 \%$ at $7.7 \mathrm{~mol}$ phenol/mol DPPH after 60 min. $^{148}$

Although ARs do not possess a hydroxyl group in the ortho-position, another factor that can affect the efficiency of an antioxidant for fat systems is the lipophilicity, which allows better penetration in the lipids micelles. A comparison between the relative antioxidant activities (RAA) of different phenolic compounds in a test system including linoleic acid micelles stabilized with $\mathrm{Na}$ dodecyl sulfate (pH 7.4, Temp. $50{ }^{\circ} \mathrm{C}$ ) showed ferulic acid and caffeic acid (with OH-group in ortho-position) cannot be considered as an effective antioxidant under these conditions since they cannot penetrate through lipid micelles. ${ }^{26}$

Our results showed that the AR molar ratio in wheat bran extract used for the DPPH assay ranged from $0.02-3.6 \mathrm{~mol} / \mathrm{mol} \mathrm{DPPH}$, with the lowest ratio belonging to Harvard cultivar from Palmerstone region and the highest Emmit cultivar from Ridgetown region. Comparing these ratios with the AR standards and their antioxidant 
activity, it can be concluded that other phenolic compounds in the extract such as ferulic acid, have more pronounced effects on the overall antioxidant capacity of wheat bran when compared to ARs.

\subsubsection{ORAC assay of wheat bran extracts}

ORAC measures antioxidant inhibition of peroxyl radicals and reflects classical radical chain-breaking antioxidant activity by $\mathrm{H}$-atom transfer. ${ }^{110}$ The peroxyl radical generated from thermal decomposition of AAPH in aqueous buffer reacts with a fluorescent probe to form a non-fluorescent product. Higher ORAC values indicate less fluorescent decay over a certain period of time.

ORAC values of all wheat bran-acetone extracts ranged between 6 and $94 \mu$ mole $\mathrm{TE} / \mathrm{g}$ (trolox equivalent) as shown in Table 2.5. ORAC values were significantly different $(P<0.001)$ for region and cultivar and their interaction (Table 2.4). The wheat bran cultivars grown in Ridgetown and Bath locations showed the highest and lowest respective antioxidant capacities. Although differences in ORAC value among cultivars were not significant, AC Morley had the lowest ORAC value. The correlation between total AR contents and ORAC values were significantly related $\left(R^{2}=0.78, P<0.05\right)$. Also a small but significant correlation $\left(R^{2}=0.41, P<0.05\right)$ was found between two employed antioxidant assays.

Overall, ORAC values of soft wheat (50 $\mu$ mole TE/g) were higher than hard wheat (37 $\mu$ mole TE/g). Moore et al. reported the ORAC of eight Maryland-grown soft wheat samples and ORAC values ranged from 33 to $47 \mu$ mole TE/g. ${ }^{149}$ Another study also reported the ORAC values of six different whole wheat varieties to be between 20 to 37 $\mu$ mole TE/g. ${ }^{150}$ 
Table 2-6 Antioxidant assays of individual and mixture of ARs standard

\begin{tabular}{cccc}
\hline AR Standards & $\begin{array}{c}\text { Concentration in mole } \\
(\text { phenol/ DPPH) }\end{array}$ & \%DPPH* & ORAC $(\mu$ moleTE/g)* \\
\hline C15:0 & 5.2 & $19.2 \pm 1.0$ & $199.4 \pm 3.8$ \\
C17:0 & 4.8 & $23.0 \pm 0.6$ & $202.1 \pm 5.3$ \\
C19:0 & 4.4 & $20.3 \pm 2.0$ & $228.1 \pm 7.6$ \\
C21:0 & 4.1 & $20.7 \pm 1.3$ & $142.8 \pm 4.3$ \\
C23:0 & 3.8 & $20.9 \pm 0.0$ & $178.2 \pm 5.6$ \\
C25:0 & 3.6 & $20.1 \pm 1.7$ & $215.0 \pm 9.8$ \\
ARs-mix $^{\text {a }}$ & 4.3 & $31.5 \pm 1.8$ & $245.9 \pm 7.6$ \\
\hline
\end{tabular}

\footnotetext{
a ARs-mix comprises of all individual AR in gram ratio similar to their ratio in wheat bran extract and the mol presented in the Table is the sum of their individual calculated mole

${ }^{*}$ Values are means of triplicates \pm standard deviations (SD), \%DPPH $=1$, 1-diphenyl-2-picrylhydrazyl as $\%$ inhibition after $30 \mathrm{~min}$, ORAC $=$ Oxygen Radical Absorbance Capacity as $\mu$ mole TE (Trolox Equivalent) / g
}

The ORAC values for AR standards are presented in Table 2.6. There were only minor differences between AR standards, and a mixture of ARs standards showed higher antioxidant activity than individual ARs as was found with DPPH assay results.

\subsection{Conclusion}

The current study investigated the effects of region and cultivar on total AR content, relative composition analysis, as well as total phenolic count and antioxidant activity of wheat bran extracts. The results showed that the Ridgetown region has greater potential for varieties rich in AR content. The highest amount of ARs was found in cultivar Emmit and the most common AR homologues in wheat brans were C21:0, followed by C19:0, C23:0, C25:0 and C17:0. TPC and antioxidant activity of wheat bran extracts were significantly affected by location and cultivar and their interaction. Our work provides a detailed examination of region and cultivar effects on AR content in 
wheat bran and presents a prospective for producing wheats rich in ARs. These results can be used for screening, breeding and genetic modification purposes. Thoroughly designed animal and pilot human studies are needed to investigate the bioavailability of wheat ARs from different wheat-based food products. 


\subsection{Connecting Statement to Chapter 3}

Chapter 2 provided a detailed examination of region and cultivar effects on potential of ARs in wheat bran and the results can be used for screening and breeding purposes.

In Chapter 3, the stability of natural levels of ARs in baked breads was investigated by analyzing and comparing total and homologue AR levels using GC-MS. Also, the study aimed to determine how the baking process affects the TPC and antioxidant activity of ARs by measuring ORAC and DPPH inhibition.

The work presented in Chapter 3 has been used to prepare two conference presentations (oral) and one publication, as follows.

- Gunenc, A. and Hosseinian, F., 2011. Potential of alkylresorcinols in Canadian red hard and red soft wheat bran and their stability during baking, American Association of Cereal Chemists (AACC)-International Conference, Palm Springs, CA, USA, October 16-19.

- Gunenc, A., Seetharaman, K., Mayer, P. Fairbanks, D., and Hosseinian, F., 2011. Potential of alkylresorcinols in Canadian red hard and red soft wheat bran and their stability during baking, Canadian Section of American Oil Chemists'Society (CAOCS), 24th Conference on Fats and Oils, Edmonton, AB, Canada, September 26-27.

- Gunenc, A., Tavakoli, H., Seetharaman, K., Mayer, P.M., Fairbanks, D. and Hosseinian, F., 2013. Stability and antioxidant capacity of alkylresorcinols in Canadian hard and soft red wheat bran during baking, Food Research International, 51:571-578. 


\section{Chapter 3 Stability and antioxidant activity of alkyresorcinols in breads enriched with hard and soft wheat bran}

\subsection{ABSTRACT}

Alkylresorcinols (ARs) are phenolic lipids that are mostly found in rye and wheat bran. In this study, the heat stability of ARs and the AR homologue composition of hard red wheat bran (HRWB) and soft red wheat bran (SRWB) were studied using four different bread formulations. ARs in wheat bran samples were extracted with four different solvents whereas ARs were extracted from the breads using hot 1-propanol. Gas chromatography-mass spectrometry (GC-MS) was used to quantify AR contents of wheat bran and bread samples. The different breads used in each trial were: A) $100 \%$ white flour (control breads), B) 30\% wheat bran, C) $30 \%$ residue bran after AR extraction, and D) $30 \%$ wheat bran plus $2 \%$ crude ARs extract. AR content in breads varied from 1.1 to $82.9 \mathrm{mg} / 100 \mathrm{~g}$, demonstrating that they were heat stable during baking. After $1 \mathrm{~h}$ of cooling, all breads showed significantly different $(P<0.05)$ inner temperatures, heights, and weights from that of the control bread. Furthermore, bread D displayed an improved height than breads $\mathrm{B}$ and $\mathrm{C}(P<0.05)$. The effects of baking on total phenolic content (TPC) and antioxidant activity were also determined. A positive correlation was observed between oxygen radical absorbance capacity (ORAC) and TPC $\left(R^{2}=0.90\right)$. 


\subsection{Introduction}

\subsubsection{Bread, the major staple food}

Many food products such as bread, pasta, noodles, and breakfast cereal products are made from cereal grains. ${ }^{151}$ One of the most important and widely consumed foods in the world is cereal products. Among the cereals, wheat and rye are the most suitable basic ingredients for bread making. ${ }^{152,153}$ Bread consumption provides 50\% carbohydrate, 30\% protein, and $50-60 \%$ vitamin $\mathrm{B}$ of the daily requirements for people of industrialized nations. ${ }^{152}$ The main antioxidant components found in cereals are phenolic compounds such as phenolic acids, ARs, tannins, and anthyocyanins. ${ }^{154}$ Some of these phytochemical compounds may reduce the risk of many diseases such as cancer, cardiovascular disease, obesity, type 2 diabetes, cataracts, osteoporosis, and urinary tract infections. ${ }^{30,33,135,155} \mathrm{It}$ is becoming more obvious that these health benefits are related to the consumption of whole grain cereal products due to their composition of micronutrients, phytochemicals, and dietary fiber. ${ }^{30}$

\subsubsection{ARs in bread}

ARs are phenolic compounds predominantly present in the bran fractions of wheat,

rye, and triticale. ${ }^{52}$ AR content has been reported to be present in cereal brans at levels of $36-320 \mathrm{mg} / 100 \mathrm{~g}$ in rye, $32-101 \mathrm{mg} / 100 \mathrm{~g}$ in wheat, and $58-163 \mathrm{mg} / 100 \mathrm{~g}$ in triticale. ${ }^{46}$ Even though the total amount of ARs in wheat or rye varies between cereal species, the relative homologue composition of ARs remains almost the same within species. ${ }^{145}$ For instance, the relative homologue composition of ARs in wheat samples has been reported as 3-6 \% C17:0, 29-42\% C19:0, 46-55 \% C21:0, 4-12\% C23:0, and 1-4 \% C25:0. ARs are only found in the inner pericarp, hyaline layer, and testa of cereal grains. Thus a high 
amount of ARs is present in bran and very minimal levels in the endosperm. The ratio of $\mathrm{C} 17: 0 / \mathrm{C} 21: 0$ can be used to differentiate between three main cereals containing ARs such as 0.1 for wheat, 0.01 for durum wheat and 1.0 for rye. ${ }^{45}$

If wheat bran or whole grain flour is added in adequate amounts to specific food products, it can enhance potential health benefits. A substantial amount of bran addition may cause dough to get too hydrated and thus non-elastic, consequently leading to a

decrease in leavening volume and tougher more firm dough. ${ }^{153}$ Some earlier studies have indicated that fermentation and baking could decrease the amount of ARs in bread; however, later studies have shown that the problem lies in the poor extraction of ARs rather than being caused by baking. Ross et al.(2003) used different solvents to extract ARs from breads containing rye bran and showed that a mixture of hot propanol and water was the best solvent to extract ARs completely with high yield. ${ }^{38}$ The extraction conditions were hot-propanol/water $(3: 1, \mathrm{v} / \mathrm{v})$ with three fold extractions at $100{ }^{\circ} \mathrm{C}(2$ times $\mathrm{x} 2 \mathrm{~h}$ followed by 1 time $\mathrm{x} 1 \mathrm{~h}$ ) using fresh solvent each time. Currently, there is a lack of research studies on the determination of AR content and quality in Canadian wheat breads and how that is influenced during baking. Hirawan et al.(2010) studied how the effects of cooking could alter the antioxidant properties of commercial, regular and whole-wheat spaghetti. ${ }^{156}$ They found that whole wheat spaghetti brands exhibited significantly higher contents of phenolic compounds and ferulic acid in comparison to regular spaghetti brands.

\subsubsection{Objectives}

The objective of this study was to investigate the stability of natural levels of ARs in baked breads by analyzing and comparing total and individual AR homologue quantity 
using gas chromatography/mass spectrometry (GC-MS). This was accomplished by producing wheat breads of differing formulations and measuring the level of ARs in these breads after baking. Moreover, the study aimed to determine how the process of baking affects the total phenolic count (TPC) and the antioxidant activity of ARs; the antioxidant activity was measured by oxygen radical absorbance capacity (ORAC) and 2, 2-diphenyl1-picryhydrazyl radical (DPPH) radical scavenging activity assays.

\subsection{Materials and methods}

\subsubsection{Materials}

Hard red wheat bran (HRWB) and soft red wheat bran (SRWB) were obtained from Kraft-Canada. All the other ingredients needed for bread making were supplied by the Culinary Department of Algonquin College, Ottawa, Ontario, Canada. Detailed information of all chemical materials used for this study can be found in the section 2.3.2.

\subsubsection{Analysis of ARs}

\subsubsection{Extraction of ARs in wheat bran samples}

ARs from two WB samples were extracted by ethanol in a ratio of 1 to $40(\mathrm{w} / \mathrm{v})$ according to the methods of Mullin et al. ${ }^{87}$ After $24 \mathrm{~h}$ of stirring at room temperature, the samples were filtered using double Whatman filter paper. The precipitate was discarded and the solvents were evaporated from the supernatant using a Rotavapor (BuchiBrinkman, R110, Switzerland) and the dried product was weighed and stored at $-20{ }^{\circ} \mathrm{C}$ until it was ready to be used in bread trial D. The residues of HRWB and SRWB after ethanol extraction were dried at room temperature and used for bread trial $\mathrm{C}$ which was based on $30 \%$ extracted wheat bran (EWB). Also, the same extraction method was 
repeated using acetone, ethyl acetate and 1-propanol to compare ARs content and composition. All analyses were performed in triplicates.

\subsubsection{Extraction of ARs in bread samples}

The freeze-dried bread samples were crushed and milled to a particle size of less than $0.5 \mathrm{~mm}$. The bread samples were extracted with 1-propanol and water in accordance

with Ross et al. ${ }^{38}$ The milled samples $(0.5 \mathrm{~g}$ for breads B and D and $1 \mathrm{~g}$ for breads A and C) were placed in $30 \mathrm{ml}$ glass tubes and capped tightly. Later, $200 \mu \mathrm{l}$ of internal standard $(0.5 \mathrm{mg} / \mathrm{ml}, \mathrm{C} 22: 0)$ and $10 \mathrm{ml}$ of 1-propanol: water $(3: 1, \mathrm{v} / \mathrm{v})$ were added to the tubes and placed in a boiling water bath for 2 hours while being mixed by vortexer every $30 \mathrm{~min}$. After allowing tubes to cool to room temperature, they were centrifuged at $2300 \mathrm{x} g$ for $10 \mathrm{~min}$; supernatants were then transferred to $50 \mathrm{ml}$ tubes and capped tightly. The same extraction procedure was repeated two more times. ${ }^{38}$ Eventually, all the supernatants from the repeated extractions were collected and combined in one tube. Later, $4 \mathrm{ml}$ portions of each sample extract were dried under nitrogen stream. All analyses were performed in triplicate.

\subsubsection{GC-MS analysis}

ARs in all extracted samples were analyzed by GC-MS and all detailed information about analyzing conditions is in section 2.3.4.

\subsubsection{Bread making procedure}

\subsubsection{Bread preparation}

In this study, seven different bread trials have been prepared using white flour (as a negative control) and wheat brans (HRWB and SRWB) according to a modified method ${ }^{52}$ 
of Andersson et al.(2010). The four different trials of bread were baked with: A: $100 \%$ white flour (WF or Control Bread); B: 30\% non-extracted wheat bran (NEWB); C: 30\% extracted wheat bran (EWB); and D: $30 \%$ (NEWB) plus crude $2 \%$ crude AR extract. David Fairbanks who is the chef from Algonquin College baked the breads according to the recipe shown in the Table 3.1. All analyses were performed in triplicate.

Table 3-1 All bread trials and recipes for bran samples: HRWB (hard red wheat bran) and SRWB (soft red wheat bran)

\begin{tabular}{lcc}
\hline $\begin{array}{l}\text { Bread } \\
\text { types }\end{array}$ & Bread trials $^{\text {a }}$ & Bread recipes \\
\hline $\mathrm{A}$ & $70 \% \mathrm{WF}+30 \% \mathrm{WF}$ & $140 \mathrm{~g} \mathrm{WF}+60 \mathrm{~g} \mathrm{WF}+144 \mathrm{~g}$ water $+1.9 \mathrm{~g}$ salt +2.5 g yeast \\
$\mathrm{B}$ & $70 \% \mathrm{WF}+30 \% \mathrm{NEWB}$ & $140 \mathrm{~g} \mathrm{WF}+60 \mathrm{~g} \mathrm{NEWB}+144 \mathrm{~g}$ water $+1.9 \mathrm{~g}$ salt $+2.5 \mathrm{~g}$ yeast \\
& & \\
$\mathrm{C}$ & $70 \% \mathrm{WF}+30 \% \mathrm{EWB}$ & $140 \mathrm{~g} \mathrm{WF}+60 \mathrm{~g} \mathrm{EWB}+144 \mathrm{~g}$ water $+1.9 \mathrm{~g}$ salt $+2.5 \mathrm{~g}$ yeast \\
$\mathrm{D}$ & $70 \% \mathrm{WF}+30 \% \mathrm{NEWB}$ & $140 \mathrm{~g} \mathrm{WF}+60 \mathrm{~g} \mathrm{NEWB}+4 \mathrm{~g}$ crude ARs extract $+144 \mathrm{~g}$ water + \\
& $+2 \%$ crude ARs extract & $1.9 \mathrm{~g} \mathrm{salt}+2.5$ g yeast \\
\hline
\end{tabular}

${ }^{\mathrm{a}} \mathrm{EWB}=$ Extracted wheat bran, NEWB $=$ Non-extracted wheat bran, WB $=$ Wheat Bran, WF $=$ White Flour

\subsubsection{Baking procedure}

The bread making process included 5 steps: 1) mixing, 2) fermentation, 3) makeup, 4) proofing and 5) baking (Figure.3.1). The baking dough was prepared on a flour weight basis with $200 \mathrm{~g}$ flour mixture, $2.5 \mathrm{~g}$ dry yeast (Saf-Instant, lesouffre yeast corporation, Milwaukie, Wisconsin, U.S.A.), 1.9 g sodium chloride (Windsor Table Salt, The Canadian Salt Company Limited, Point-Claire, Quebec, Canada), and tap water $\left(48^{\circ} \mathrm{C}\right)$. The ingredients were mixed as a straight dough method for 10 minutes in a countertop mixer (Hobart Legacy, Hobart Corporation, Troy, $\mathrm{OH}$ ) at $2^{\text {nd }}$ speed and the doughs were 
left in a leavening cupboard (Metro proofer, Inter-metro industries corporation, California, USA) at $34^{\circ} \mathrm{C}$ at $60 \%$ Relative Humidity (RH) for $60 \mathrm{~min}$. The doughs were then divided into 3 pieces $(3 \times 100 \mathrm{~g})$, molded, and placed in baking loaf pans laced in non-grazed molds, then placed in a second leavening cupboard at $39^{\circ} \mathrm{C}$ at $85 \% \mathrm{RH}$ for 60 $\min$. The fermented dough was baked for $12 \mathrm{~min}$ at $255^{\circ} \mathrm{C}$ in a rotation oven (GarlandMaster 200, USA). The inner temperature, as well as the weight and height of the breads were measured after 60 minutes of cooling. The breads were put in sealed plastic bags, frozen and freeze-dried for further analysis.

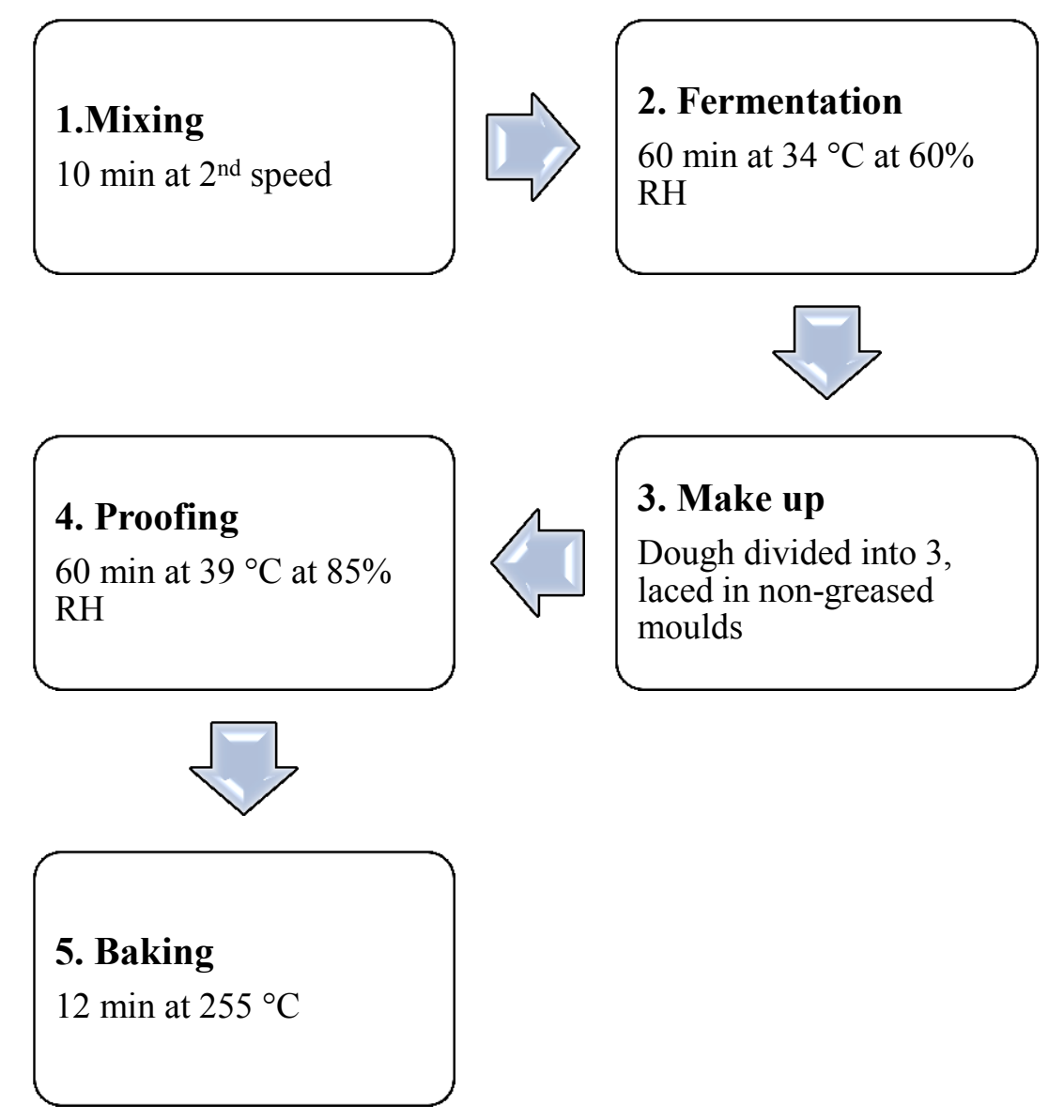

Figure 3-1 The bread making procedure 


\subsubsection{Antioxidant activity}

\subsubsection{Sample extraction}

For the extraction procedure, $15 \mathrm{~mL}$ of ethanol (95\%): $1 \mathrm{~N} \mathrm{HCl}(85: 15, \mathrm{v} / \mathrm{v})$ was mixed with $1.0 \mathrm{~g}$ of milled sample in $50 \mathrm{~mL}$ brown bottles and was continuously shaken for $6 \mathrm{~h}$ at $300 \mathrm{rpm}$ and at an ambient temperature. The mixture was then centrifuged at 7,800 x g (Thermo Scientific, Sorval Legend XTR) at $5^{\circ} \mathrm{C}$ for $15 \mathrm{~min}$. The resulting supernatant fluid was then stored at $-20^{\circ} \mathrm{C}$ in the dark, and kept till further analysis of TPC, DPPH, and ORAC ${ }^{142}$. All analyses were performed in triplicates.

TPC, DPPH and ORAC measurement of WB and bread samples were performed by following sections $2.3 .5,2.3 .6$ and 2.3 .7 , respectively.

\subsubsection{Statistical Analysis}

All statistical analyses were performed, using SAS version 9.1 (SAS Institute Inc., Cary, NC, USA). Analysis of variance (ANOVA) using Fisher t-test, LSD was used to determine if there was a significant difference $(P<0.05)$ among triplicates of total and homologue of ARs before and after baking. Also the same analysis was performed to see if there was significant difference $(P<0.05)$ between ORAC, DPPH and TPC data sets.

\subsection{Results and Discussion}

\subsubsection{AR content and homolog composition in wheat bran}

GC-MS analysis was used to measure the total content of saturated, unsaturated ARs (mg/100g), and level of individual homologues (\%) in wheat bran samples. The saturated ARs were confirmed by comparison of retention times of AR standards (C15:0$\mathrm{C} 25: 0)$ and their molecular ion mass to charge ratio (m/z) as follows, 464 (C15:0), 492 
(C17:0), 520 (C19:0), 548 (C21:0), 576 (C23:0), and 604 (C25:0). The main fragmentation ions were observed at $\mathrm{m} / \mathrm{z} 268,267$, and 281 ; this is most likely due to McLafferty rearrangement, $\beta$ cleavage and $\gamma$ cleavage, respectively. The peaks with these characteristic $\mathrm{m} / \mathrm{z}$ values were considered resorcinolic derivatives and this is in agreement with results reported in literature. ${ }^{34,35,140}$

Table 3.2 shows the AR content and composition in bran samples. The wheat bran samples were HRWB, SRWB, HRWB-R, SRWB-R and WF (control). Four different solvents including acetone, ethanol, ethyl acetate and 1-propanol were used to extract the above WB samples. The effect of various extraction solvents on AR content $(\mathrm{mg} / 100 \mathrm{~g})$ in WB samples is shown in Table 3.2. 


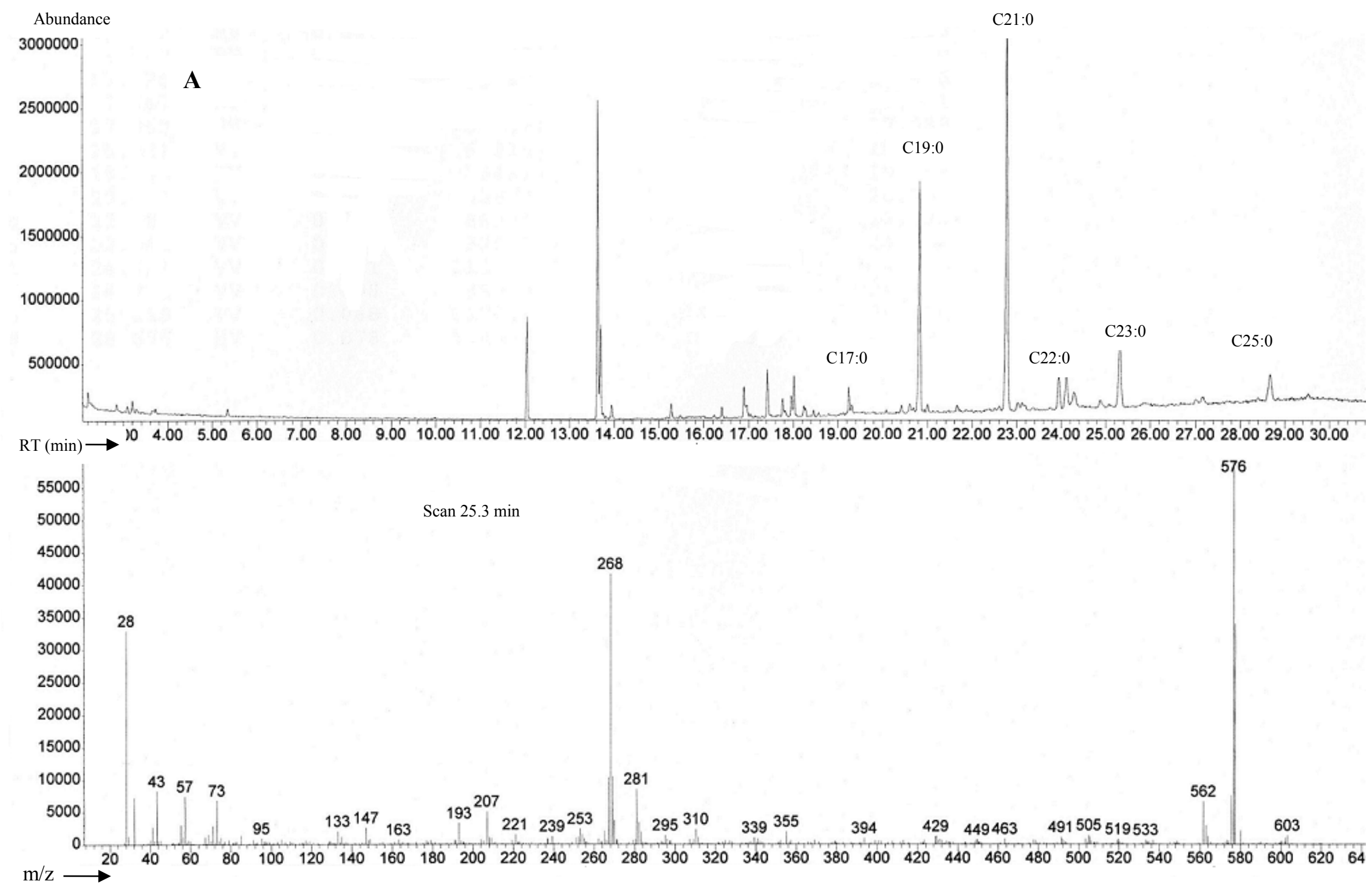




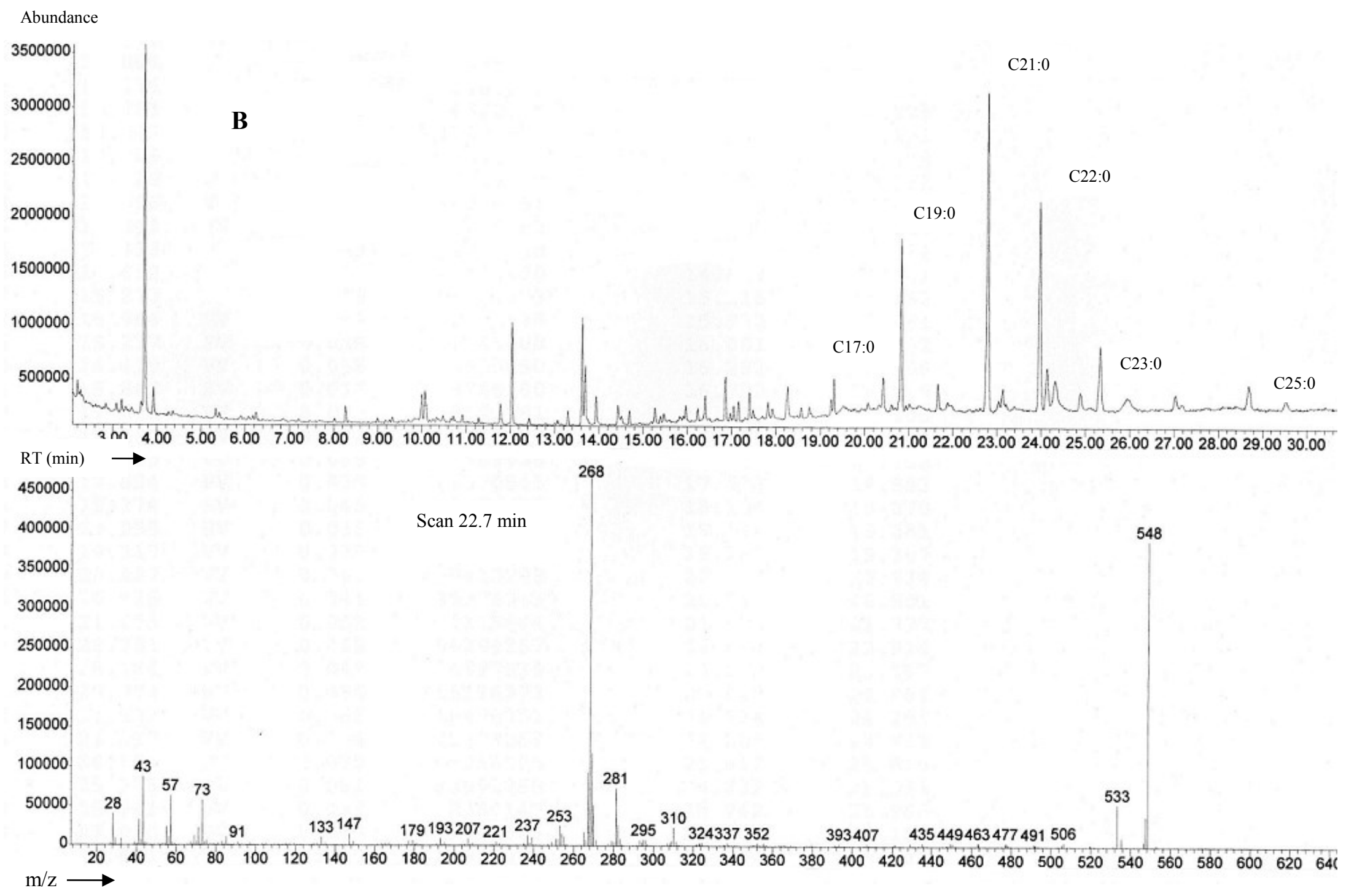

Figure 3-2 GC-MS results of ARs from HRWB before baking (A), and in bread 1D (30\%wheat bran plus 2\% crude ARs extract) (B) 
Table 3-2 Total AR content (mg/100 g), \% saturated (Sat), unsaturated (Unsat) and homologue (C15-C25) ARs in wheat bran, bran-residue and white flour.

\begin{tabular}{|c|c|c|c|c|c|c|c|c|c|}
\hline Sample $^{*}$ & Extraction solvent & Total-ARs $^{* *}$ & Sat.AR & Unsat.AR & $\mathrm{C} 17$ & C19 & $\mathrm{C} 21$ & $\mathrm{C} 23$ & $\mathrm{C} 25$ \\
\hline HRWB & Acetone & $282.6 \pm 3.9^{\mathrm{a}}$ & $94.1 \pm 0.7^{\mathrm{ab}}$ & $5.9 \pm 0.7^{b}$ & $2.0 \pm 0.1^{\mathrm{de}}$ & $25.3 \pm 1.6^{b}$ & $53.2 \pm 2.1^{b c}$ & $9.6 \pm 0.24^{\mathrm{def}}$ & $3.9 \pm 0.1^{\mathrm{ef}}$ \\
\hline HRWB & Ethyl acetate & $241.8 \pm 0.7^{\mathrm{b}}$ & $91.8 \pm 1.5^{\mathrm{ab}}$ & $8.2 \pm 1.5^{b}$ & $1.8 \pm 0.2^{\mathrm{ef}}$ & $24.4 \pm 0.4^{b}$ & $51.5 \pm 0.8^{\mathrm{bcd}}$ & $9.6 \pm 0.3^{\mathrm{def}}$ & $4.4 \pm 0.7^{\mathrm{def}}$ \\
\hline HRWB & 1-propanol & $195.8 \pm 0.9^{d}$ & $93.1 \pm 0.8^{\mathrm{ab}}$ & $6.9 \pm 0.8^{b}$ & $2.1 \pm 0.1^{\mathrm{d}}$ & $26.1 \pm 0.5^{b}$ & $49.0 \pm 0.6^{\mathrm{cdef}}$ & $10.7 \pm 0.1^{\text {def }}$ & $5.0 \pm 0.47^{\mathrm{de}}$ \\
\hline HRWB & Ethanol & $109.5 \pm 4.0^{\mathrm{f}}$ & $95.6 \pm 0.2^{\mathrm{ab}}$ & $4.4 \pm 0.2^{b}$ & $1.8 \pm 0.1$ ef & $23.3 \pm 0.0^{b}$ & $56.1 \pm 0.6^{\mathrm{a}}$ & $6.8 \pm 3.6^{\mathrm{f}}$ & $7.5 \pm 3.4^{b}$ \\
\hline SRWB & Acetone & $241.0 \pm 2.1^{b}$ & $93.4 \pm 0.6^{\mathrm{ab}}$ & $6.6 \pm 0.6^{b}$ & $2.2 \pm 0.2^{\mathrm{d}}$ & $25.0 \pm 0.9^{b}$ & $52.3 \pm 1.4^{\mathrm{bcd}}$ & $9.8 \pm 0.6^{\mathrm{def}}$ & $4.0 \pm 0.3^{\mathrm{def}}$ \\
\hline SRWB & Ethyl acetate & $218.5 \pm 4.6^{\mathrm{c}}$ & $92.2 \pm 0.3^{b}$ & $7.8 \pm 0.3^{b}$ & $1.7 \pm 0.1^{\text {ef }}$ & $23.3 \pm 0.0^{b}$ & $53.1 \pm 0.2^{b c}$ & $9.8 \pm 0.0^{\mathrm{def}}$ & $4.2 \pm 0.1^{\mathrm{de}_{f}}$ \\
\hline SRWB & 1-propanol & $187.5 \pm 10.4^{\mathrm{e}}$ & $92.7 \pm 0.1^{\mathrm{ab}}$ & $7.3 \pm 0.1^{b}$ & $1.8 \pm 0.1{ }^{\text {ef }}$ & $24.7 \pm 0.9^{b}$ & $48.9 \pm 1.2^{\mathrm{cdef}}$ & $11.7 \pm 0.1^{\text {def }}$ & $5.5 \pm 0.3^{\mathrm{cd}}$ \\
\hline SRWB & Ethanol & $68.6 \pm 2.3^{\mathrm{g}}$ & $92.2 \pm 0.2^{b}$ & $7.8 \pm 0.2^{b}$ & $3.2 \pm 0.1^{\mathrm{b}}$ & $31.4 \pm 0.4^{\mathrm{a}}$ & $45.5 \pm 0.4^{\mathrm{ef}}$ & $8.5 \pm 0.1^{\text {ef }}$ & $3.5 \pm 0.1^{\mathrm{f}}$ \\
\hline HRWB-R & Acetone & $46.8 \pm 3.8^{h}$ & $100^{\mathrm{a}}$ & nd & $3.0 \pm 0.1^{\mathrm{b}}$ & $25.8 \pm 0.0^{b}$ & $50.3 \pm 0.9^{\text {cde }}$ & $14.3 \pm 0.2^{\mathrm{cd}}$ & $6.6 \pm 0.5^{b c}$ \\
\hline HRWB-R & Ethyl acetate & $36.9 \pm 1.1^{\mathrm{i}}$ & $100^{\mathrm{a}}$ & nd & $2.2 \pm 0.4^{\mathrm{d}}$ & $24.9 \pm 0.3^{b}$ & $50.5 \pm 1.3^{\text {cde }}$ & $15.0 \pm 1.1^{\mathrm{cd}}$ & $7.4 \pm 0.2^{b}$ \\
\hline HRWB-R & 1-propanol & $34.5 \pm 1.5^{\mathrm{ij}}$ & $100^{\mathrm{a}}$ & nd & nd & nd & $27.4 \pm 0.9^{h}$ & $54.9 \pm 1.3^{b}$ & $17.7 \pm 0.4^{\mathrm{a}}$ \\
\hline HRWB-R & Ethanol & $14.2 \pm 0.5^{l}$ & $100^{\mathrm{a}}$ & nd & $2.6 \pm 0.2^{c}$ & $25.4 \pm 0.2^{b}$ & $50.4 \pm 1.1^{\mathrm{cde}}$ & $14.6 \pm 0.6^{\mathrm{cd}}$ & $6.9 \pm 0.2^{b}$ \\
\hline SRWB-R & Acetone & $20.1 \pm 0.5^{\mathrm{k}}$ & $82.6 \pm 1.8^{\mathrm{c}}$ & $17.4 \pm 0.0^{\mathrm{a}}$ & nd & $23.2 \pm 5.3^{b}$ & $44.9 \pm 9.3^{\mathrm{f}}$ & $14.3 \pm 2.8^{\mathrm{cd}}$ & nd \\
\hline SRWB-R & Ethyl acetate & $14.2 \pm 0.3^{l}$ & $100^{\mathrm{a}}$ & nd & nd & $26.4 \pm 6.8^{b}$ & $54.8 \pm 5.8^{a b}$ & $18.7 \pm 1.4^{\mathrm{cd}}$ & nd \\
\hline SRWB-R & 1-propanol & $18.9 \pm 1.9^{\mathrm{kl}}$ & $100^{\mathrm{a}}$ & nd & nd & nd & $33.6 \pm 1.1^{\mathrm{g}}$ & $66.3 \pm 1.1^{\mathrm{a}}$ & nd \\
\hline SRWB-R & Ethanol & $4.1 \pm 0.1 \mathrm{mn}$ & $100^{a}$ & nd & nd & $24.8 \pm 1.6^{b}$ & $55.9 \pm 1.1^{\mathrm{ab}}$ & $19.2 \pm 0.5^{\mathrm{c}}$ & nd \\
\hline WF & Acetone & $8.6 \pm 0.4^{\mathrm{m}}$ & $78.1 \pm 1.1^{\mathrm{c}}$ & $21.9 \pm 1.1^{\mathrm{a}}$ & nd & $30.4 \pm 1.1^{\mathrm{a}}$ & $47.5 \pm 1.0^{\operatorname{def}}$ & nd & nd \\
\hline WF & Ethyl acetate & $\mathrm{nd}^{* * *}$ & nd & nd & nd & nd & nd & nd & nd \\
\hline WF & 1-propanol & nd & nd & nd & nd & nd & nd & nd & nd \\
\hline WF & Ethanol & $30.2 \pm 0.5^{j}$ & $100^{\mathrm{a}}$ & nd & $4.8 \pm 0.2^{\mathrm{a}}$ & $31.1 \pm 0.3^{\mathrm{a}}$ & $47.4 \pm 0.0^{\mathrm{def}}$ & $12.0 \pm 0.0^{\mathrm{def}}$ & $4.6 \pm 0.2^{\mathrm{def}}$ \\
\hline
\end{tabular}

${ }^{*} \mathrm{HRWB}=$ hard red wheat bran, SRWB $=$ soft red wheat bran, HRWB-R= hard red wheat bran-residue, SRWB-R $=$ soft red wheat bran-residue, WF= white flour.

${ }^{* *}$ Values are means of triplicates \pm standard deviations (SD). Values with different letters in columns are significantly different from each other at $P<0.05$. Correlation of variation $(\mathrm{CV} \%)$ range is 3.54 . ${ }^{* * *} \mathrm{nd}=$ not detected. 
There was a significant difference $(P<0.05)$ in the total AR content extracted by the four different solvents used in each trial. The highest yield was recorded for acetone, and in addition it was found to be a more selective solvent for AR extracts compared to other solvent systems. ${ }^{155}$ For the WB samples, the order of the solvents giving the highest to lowest AR yield was acetone $>$ ethyl acetate $>1$-propanol $>$ ethanol.

Although the total AR content was different for each WB sample, the relative $\%$ saturated and unsaturated ARs for HRWB remained close to that of SRWB and our findings is comparable to the values reported in literature. ${ }^{34,35,140}$

It has been suggested that ARs may have some inhibitory effects on metabolic enzymes. The length of the alkyl chain affects the extend of inhibition, with C17:0 and C19:0 being the most effective inhibitors. ${ }^{94}$ ARs were also able to prevent triglyceride accumulation in cultured 3T3-L1 cells, indicating that they may inhibit triglyceride synthesis in vivo. ${ }^{94}$ It has been reported by Ross et al.(2004) that wheat bran has minor AR derivatives including ARs with unsaturated alkyl chains, keto groups, or a combination of both keto groups and unsaturated alkyl chains. ${ }^{34}$ These minor AR derivatives are claimed to have many specific biological activities that are related to their amphiphilic characteristics. ${ }^{157,158}$ Unsaturated ARs may affect cell membrane permeability and prevent LDL oxidation in humans, similar functionality to polyunsaturated fatty acids. ${ }^{159}$ A thorough explanation of the chemistry, nutritional effects, and bioactivities of ARs is discussed in more detail in reviews by Kozubek and $\operatorname{Tyman}^{33}$ and Ross et al. ${ }^{34}$ The unsaturated peak values were assigned according to the MS data obtained from each GC-MS run and then compared with literature data. ${ }^{34}$ Further studies need to be carried out on whether ARs have any bioactivity in higher 
mammals.

Acetone extracts showed significantly higher $(P<0.05)$ AR content and both of the wheat bran samples had very close AR homologue composition. The order of individual AR homologues from highest to lowest was $\mathrm{C} 21: 0>\mathrm{C} 19: 0>\mathrm{C} 23: 0>\mathrm{C} 25: 0>$ C17:0. In this study, the relative AR homologue composition for both WB samples was determined to be 1.8-3.2 \% for C17:0, 23.3-31.4\% for $\mathrm{C} 19: 0,45.5-56.1 \%$ for $\mathrm{C} 21: 0,6.8$ $10.7 \%$ for $\mathrm{C} 23: 0$, and $3.5-7.5 \%$ for $\mathrm{C} 25: 0$. Our findings were close to those ranges and C21:0 and C19:0 were the dominant AR homologues in the WB samples which is in agreement with other reports in literature such as those of Knodler et al. ${ }^{145}$ Meanwhile, for the residue brans, the homologue composition was not similar before and after extractions. Some AR homologues such as C17:0 and C25:0 were not found in the extraction of the residue brans. Both of these homologues were also found in the lowest amounts in regular bran. The difference in AR content between extracted and regular bran was high and our findings were parallel to the work of Hedkvist. ${ }^{160}$ In the study, they also found ARs in the acetone extract of residue rye bran $(41-57 \mathrm{mg} / 100 \mathrm{~g}) .{ }^{160}$

The WF samples yielded only a small amount of ARs, $8.6 \mathrm{mg} / 100 \mathrm{~g}$ in acetone and $30.2 \mathrm{mg} / 100 \mathrm{~g}$ in ethanol extracts (Table 3.2) that was consistent with the report by Hengtrakul et al. ${ }^{144}$ The low residual amounts might due to improper separation of the bran, resulting in having ARs in the white flour sample. ${ }^{144}$ 
Table 3-3 Total content (mg/100 g), saturated (Sat), unsaturated (Unsat) and homologue composition (\%) of ARs in all bread trials

\begin{tabular}{|c|c|c|c|c|c|c|c|c|}
\hline Bread Coding $^{*}$ & Total AR ${ }^{* *}$ & Sat.AR & Unsat.AR & $\mathrm{C} 17: 0$ & C19:0 & $\mathrm{C} 21: 0$ & $\mathrm{C} 23: 0$ & $\mathrm{C} 25: 0$ \\
\hline A & $1.1 \pm 0.6^{\mathrm{f}}$ & $100^{\mathrm{a}}$ & $\mathrm{nd}^{* * * *}$ & nd & nd & $100^{\mathrm{a}}$ & nd & nd \\
\hline 1B & $51.4 \pm 0.1^{\mathrm{c}}$ & $90.6 \pm 0.2^{c}$ & $9.4 \pm 0.2^{\mathrm{a}}$ & $2.1 \pm 0.8^{\mathrm{a}}$ & $23.1 \pm 0.5^{\mathrm{c}}$ & $45.4 \pm 1.7^{\mathrm{f}}$ & $13.3 \pm 0.3^{b}$ & $6.8 \pm 0.3^{b}$ \\
\hline $1 \mathrm{C}$ & $7.7 \pm 1.2^{\mathrm{e}}$ & $100^{\mathrm{a}}$ & nd & nd & $27.6 \pm 0.3^{b}$ & $72.4 \pm 0.3^{b}$ & nd & nd \\
\hline $1 \mathrm{D}$ & $82.9 \pm 2.0^{\mathrm{a}}$ & $91.3 \pm 0.4^{\mathrm{b}}$ & $8.6 \pm 0.4^{b}$ & $1.6 \pm 0.1^{\mathrm{a}}$ & $22.2 \pm 0.8^{\mathrm{d}}$ & $49.3 \pm 2.3^{\mathrm{d}}$ & $12.7 \pm 0.2^{\mathrm{c}}$ & $5.6 \pm 0.6^{\mathrm{c}}$ \\
\hline $2 \mathrm{~B}$ & $39.6 \pm 6.3^{d}$ & $90.6 \pm 0.2^{\mathrm{c}}$ & $9.4 \pm 0.1^{\mathrm{a}}$ & $0.8 \pm 0.3^{b}$ & $22.2 \pm 0.1^{\mathrm{d}}$ & $46.4 \pm 0.3^{\text {ef }}$ & $13.8 \pm 0.7^{\mathrm{a}}$ & $7.3 \pm 0.3^{\mathrm{a}}$ \\
\hline $2 \mathrm{C}$ & $9.6 \pm 0.5^{\mathrm{e}}$ & $100^{a}$ & nd & nd & $32.5 \pm 0.8^{\mathrm{a}}$ & $67.7 \pm 0.6^{c}$ & nd & nd \\
\hline $2 \mathrm{D}$ & $76.9 \pm 0.9^{b}$ & $90.8 \pm 0.1^{\mathrm{c}}$ & $9.2 \pm 0.1^{\mathrm{a}}$ & $2.0 \pm 0.2^{\mathrm{a}}$ & $21.7 \pm 0.1^{\mathrm{d}}$ & $47.7 \pm 0.1^{\mathrm{de}}$ & $12.8 \pm 0.3^{\mathrm{c}}$ & $6.4 \pm 0.2^{b}$ \\
\hline
\end{tabular}

${ }^{*} 1=$ hard red wheat bran (HRWB), $2=$ soft red wheat bran (SRWB), A = control, B $=30 \%$ non-extracted bran added, $\mathrm{C}=30 \%$ extracted bran added, $\mathrm{D}=30 \%$ non-extracted bran $+2 \%$ crude ARs extract added.

${ }^{* *}$ Values are means of triplicates $\pm \mathrm{SD}$. Values with different letters in columns are significantly different from each other at $(P<0.05)$. CV \% is 7.15 .

${ }^{* *}$ nd $=$ not detected 


\subsubsection{AR content and homologue composition in bread samples}

In this study, four different solvents including acetone, ethanol, ethyl acetate and 1-propanol, were used to extract ARs from wheat bran samples but in order to extract ARs from wheat bran and add to bread samples, ethanol was used since it is the only food grade solvent that commonly applies in food industries. On the other hand, a mixture of hot propanol and water was used to extract ARs from bread samples. Ross et al.(2003) used different solvents to extract ARs from breads containing rye bran and showed that a mixture of hot propanol and water was the best solvent to extract ARs completely with high yield and release the ARs bound from starch-lipid complexes. ${ }^{38}$ The total AR and related homologue content of all breads trials were analyzed using GC-MS (Figure.3.2B) and the results are shown in Table 3.3. All breads tested in this study, contained ARs and it was shown that AR was not degraded during baking which is in agreement with literature ${ }^{38,52}$ as was expected. ${ }^{34} 42$

A significant difference $(P<0.05)$ was seen in the total amount of ARs yielded from the WB samples. The highest amount of ARs yielded from the trials was from bread D, followed by B, C, and A which was the control that was expected to have the lowest yield.

Meanwhile, when it came to the homologue AR composition in breads, B and D had very similar values before the baking was carried out. Interestingly, after the baking process, the homologue composition of breads B and D had hardly been affected as there was only a slight decrease in $\mathrm{C} 21: 0$ and C19:0 while there was an actual increase in C23:0 and C25:0 in bread B. However, the AR homologue content of breads A and C did change after baking. 
Overall, the homologue ARs content had not been significantly altered for both WB samples during baking and only a slight decrease was observed in C21:0 and C25:0 content of bread $\mathrm{D}$, as opposed to an increase in its $\mathrm{C} 23: 0$ homologue content.

\subsubsection{Effects of ARs on bread physical quality}

After baking, the breads were cooled to room temperature under a baking cloth for around $1 \mathrm{~h}$. Then the inner temperatures, heights, and weights of breads were recorded. All treatments were significantly different $(P<0.05)$ in temperature, height and weight from control bread (Table 3.4). The height differences of bread B (containing WB) and C (containing residue bran) were not significant $(P>0.05)$. Meanwhile bread D (containing highest amount of ARs) showed a significant difference $(P<0.05)$ in height in comparison with B and C breads (Table 3.4). This study suggests that ARs may have potential in bread manufacturing by improving the quality of bread. The control bread (A) had the lowest inner temperature $\left(35.6 \pm 1.3{ }^{\circ} \mathrm{C}\right)$ and greatest height $(6.8 \pm 0.1 \mathrm{~cm})$ amongst all trials. This was expected since the control bread didn't include any bran as an ingredient. Apart from the control bread, breads 1D and 2D had the greatest heights of 5.80 and $5.40 \mathrm{~cm}$, respectively. Upon an overall comparison, it was acknowledged that the breads with the highest amount of ARs were also the most elevated and raised trial breads; the ranking from highest to lowest for both HRWB and SRWB is D $>$ B $=$ C. There was a positive correlation between bread heights and AR content $\left(R^{2}=0.90\right)$. The images of each of the bread slices are shown in Figure 3.3. 
Table 3-4 Effects of ARs on bread physical quality: The inner temperature, height and weight of bread samples after $1 \mathrm{~h}$ cooling.

\begin{tabular}{llll}
\hline Bread trials $^{*}$ & Inner temperature $\left({ }^{\circ} \mathrm{C}\right)$ & Height $(\mathrm{cm})$ & Weight $(\mathrm{g})$ \\
\hline A & $35.6 \pm 1.3^{* * \mathrm{~d}}$ & $6.8 \pm 0.1^{\mathrm{a}}$ & $89.3 \pm 0.1^{\mathrm{c}}$ \\
1B & $43.9 \pm 0.3^{\mathrm{ba}}$ & $5.1 \pm 0.2^{\mathrm{d}}$ & $96.7 \pm 0.1^{\mathrm{a}}$ \\
1C & $44.9 \pm 0.9^{\mathrm{a}}$ & $4.8 \pm 0.1^{\mathrm{d}}$ & $96.7 \pm 0.1^{\mathrm{a}}$ \\
1D & $43.7 \pm 0.9^{\mathrm{ba}}$ & $5.8 \pm 0.2^{\mathrm{b}}$ & $96.7 \pm 0.1^{\mathrm{a}}$ \\
2B & $40.4 \pm 0.2^{\mathrm{c}}$ & $4.9 \pm 0.2^{\mathrm{d}}$ & $94.7 \pm 0.1^{\mathrm{b}}$ \\
2C & $42.4 \pm 0.4^{\mathrm{b}}$ & $4.8 \pm 0.1^{\mathrm{d}}$ & $96.7 \pm 0.0^{\mathrm{a}}$ \\
2D & $42.8 \pm 0.3^{\mathrm{b}}$ & $5.4 \pm 0.1^{\mathrm{c}}$ & $96.7 \pm 0.0^{\mathrm{a}}$ \\
\hline
\end{tabular}

*1 = hard red wheat bran (HRWB), 2 = soft red wheat bran (SRWB), A =control, B=30\% non-extracted bran added, $\mathrm{C}=30 \%$ extracted bran added, $\mathrm{D}=30 \%$ non-extracted bran $+2 \%$ crude ARs extract added.

\footnotetext{
${ }^{* *}$ Values are means of triplicates \pm SD. Values with different letters in columns are significantly different from each other at $(P<0.05)$. CV\% for temperature, height and weights are $1.93,0.98$, and 50 respectively.
} 


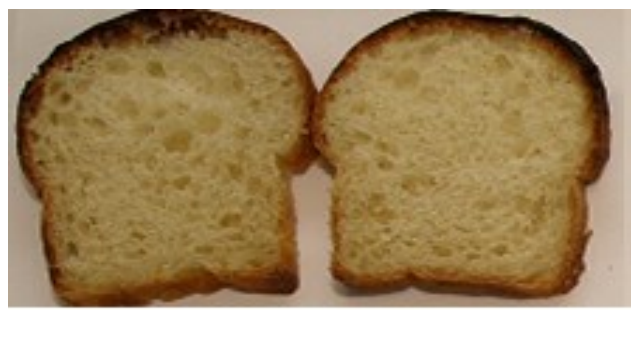

A

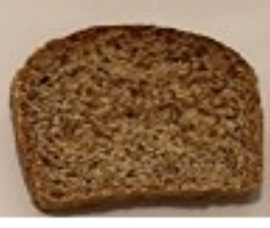

1B

2B

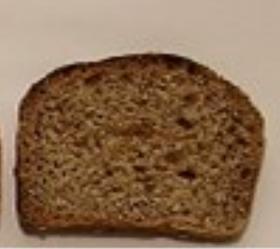

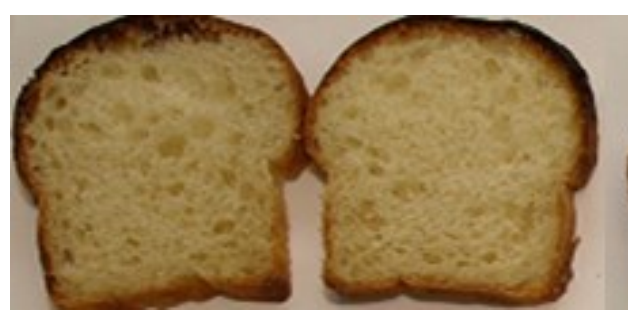

A

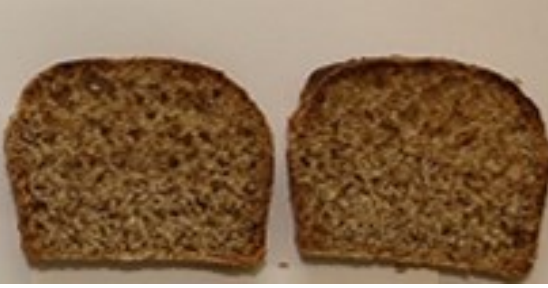

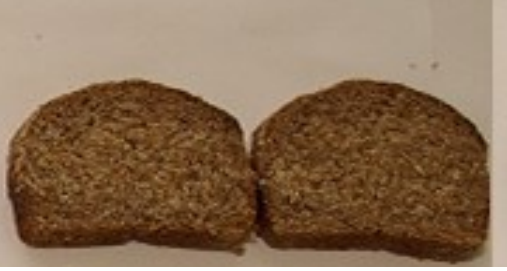

$1 \mathrm{C}$

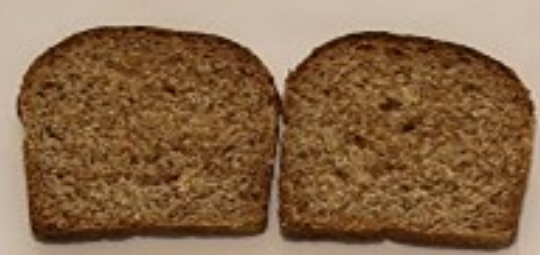

1D

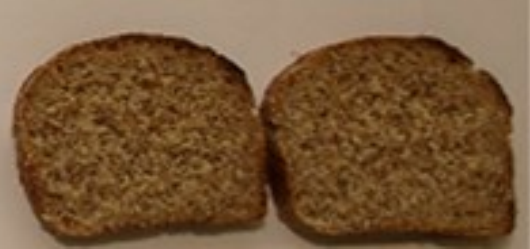

$2 \mathrm{C}$

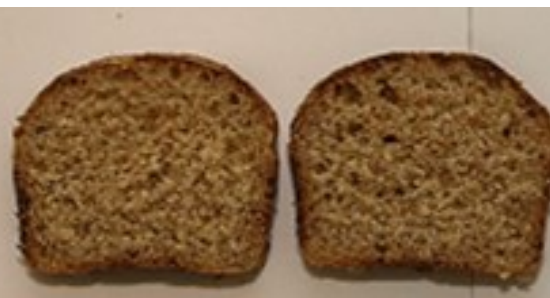

2D

Figure 3-3 Breads baked with 100\% white flour (A), 30\% non-extracted wheat bran (1B, 2B), 30\% extracted wheat bran (1C, 2C) and $30 \%$ non-extracted wheat bran and $2 \%$ crude ARs extract added (1D, 2D)

$1=$ hard red wheat bran $(\mathrm{HRWB})$ and $2=$ soft red wheat bran $(\mathrm{SRWB})$ 
Thus this specific method of AR extraction and its addition to the dough, made a significant difference perhaps due to the chemical behavior of these lipids that were added to the dough. These results showed that bread height did increase upon changing the amount of AR content. ARs are amphiphilic phenolic lipids due to the polarity of the dihydroxybenzene group and the hydrophobic alkyl carbon chain. ARs with amphiphilic properties, like emulsifiers, can act as dough softeners, changing the gelatinization behavior of starch by making complexes with amylose and as a result delay water penetration which has also been suggested to occur during baking. ${ }^{46}$ They also have the ability to travel to interfaces between two physical phases and thereby lower surface tension and form dispersions. Moreover, ARs have been suggested to form amylose-lipid complexes during baking as mentioned by Ross et al. ${ }^{38}$ This might explain the effects ARs have on the baking quality of yeast-leavened wheat breads. 


\subsubsection{TPC}

TPC was expressed as milligrams of ferulic acid equivalents per gram of sample (mg FAE/g) and the results of these measurements are shown in Table 3.5. The aim was to compare whether the phenolic content of the wheat bran had been affected during baking. For this reason, all bread ingredients, HRWB, SRWB, HRWB-R, SRWB-R and WF were studied in addition to seven types of bread trials. Before the baking trials, TPC values of both wheat bran samples (HRWB and SRWB) were significantly different $(P<$ 0.05) among residue brans and WF. Statistical analysis showed that TPC values of HRWB was $1 \mathrm{D}>1 \mathrm{~B}=1 \mathrm{C}$ and SRWB was $2 \mathrm{D}=2 \mathrm{~B}=2 \mathrm{C}$. 
Table 3-5 ORAC, \% DPPH, and TPC values of wheat bran, bran-residue, white flour and bread samples.

\begin{tabular}{clll}
\hline Sample $^{*}$ & ORAC & $\%$ DPPH & TPC \\
\hline Bran & & & \\
HRWB & $104.5 \pm 0.8^{\mathrm{b}^{* *}}$ & $29.8 \pm 3.2^{\mathrm{a}}$ & $4.9 \pm 0.3^{\mathrm{a}}$ \\
SRWB & $112.5 \pm 7.4^{\mathrm{a}}$ & $26.8 \pm 1.0^{\mathrm{a}}$ & $4.5 \pm 0.1^{\mathrm{a}}$ \\
HRWB-R & $33.2 \pm 1.6^{\mathrm{d}}$ & $21.0 \pm 2.1^{\mathrm{b}}$ & $2.0 \pm 0.0^{\mathrm{c}}$ \\
SRWB-R & $33.4 \pm 1.0^{\mathrm{d}}$ & $28.5 \pm 0.7^{\mathrm{a}}$ & $2.2 \pm 0.8^{\mathrm{bc}}$ \\
WF & $30.6 \pm 7.9^{\text {ed }}$ & $7.1 \pm 2.21^{\mathrm{d}}$ & $1.2 \pm 0.9^{\mathrm{c}}$ \\
Bread & $13.5 \pm 1.1^{\mathrm{g}}$ & $15.0 \pm 0.1^{\mathrm{c}}$ & $1.4 \pm 0.3^{\mathrm{c}}$ \\
A & $28.2 \pm 1.1^{\text {ed }}$ & $20.2 \pm 1.7^{\mathrm{b}}$ & $2.0 \pm 0.4^{\mathrm{c}}$ \\
1B & $34.8 \pm 4.4^{\mathrm{d}}$ & $19.6 \pm 1.0^{\mathrm{b}}$ & $1.5 \pm 0.3^{\mathrm{c}}$ \\
1C & $114.6 \pm 5.1^{\mathrm{a}}$ & $29.9 \pm 0.7^{\mathrm{a}}$ & $4.8 \pm 0.1^{\mathrm{ba}}$ \\
1D & $18.9 \pm 2.0^{\text {gf }}$ & $20.7 \pm 3.7^{\mathrm{b}}$ & $2.2 \pm 0.2^{\mathrm{c}}$ \\
2B & $24.8 \pm 0.4^{\text {ef }}$ & $22.8 \pm 1.9^{\mathrm{b}}$ & $1.7 \pm 0.2^{\mathrm{c}}$ \\
2C & $71.9 \pm 1.7^{\mathrm{c}}$ & $27.3 \pm 0.9^{\mathrm{a}}$ & $3.2 \pm 1.6^{\mathrm{bc}}$ \\
2D & &
\end{tabular}

ORAC $=$ Oxygen Radical Absorbance Capacity as $\mu$ mole TE (Trolox Equivalent) $/ \mathrm{g}$

$\mathrm{DPPH}=1,1$-diphenyl-2-picrylhydrazyl as \%DPPH inhibition

$\mathrm{TPC}=$ Total Phenolic Content as mg Ferulic Acid Equivalent (FAE) /g

*1 1 = HRWB (hard red wheat bran), 2 = SRWB (soft red wheat bran), $\mathrm{R}=$ residue. $\mathrm{A}=$ control, $\mathrm{B}=30 \%$ non-extracted bran added, $\mathrm{C}=30 \%$ extracted bran added, $\mathrm{D}=30 \%$ non-extracted bran $+2 \%$ crude ARs extract added

\footnotetext{
${ }^{* * *}$ Values are means of triplicates \pm SD. Values with different letters in columns are significantly different from each other at $P<0.05$. CV \% ranged between $9.63 \%$ and $16.92 \%$.
} 
The results also indicated that the effects of heat treatment on TPC of two WB samples was limited and less than $1 \mathrm{mg} / \mathrm{g}$ which is in agreement with the work of $\mathrm{Li}$ et al. ${ }^{161}$ In wheat bran, phenolic compounds are the key contributors to the total in vitro antioxidant capacity and thus a high TPC reflects a high total antioxidant activity. It is noteworthy to mention that during the extraction process, it is possible to have slight hydrolysis of polyphenolic compounds due to acidic extracting conditions thus the higher antioxidant activity. ${ }^{161}$

\subsubsection{Antioxidant activity by ORAC}

An ORAC assay is used to measure the relative potential of antioxidants which prevent biological molecules from free radical attacks. ${ }^{143,161}$ High ORAC values indicate high antioxidant capacity in a sample extract. It has been reported by Li et al ${ }^{161}$ that the ORAC values of extracts from bran and bran-containing muffins ranged from 3.0 to 25.3 $\mu$ mole $\mathrm{TE} / \mathrm{g}$. In addition, another study ${ }^{150}$ determined that the ORAC values of the free fractions of six diverse whole wheat ranged from 19.5 to $37.4 \mu$ mole TE/g. Meanwhile, the ORAC values obtained in our study were higher than both of the studies mentioned above. Furthermore, a significant correlation $(P<0.05)$ was found between TPC content and the ORAC values $\left(R^{2}=0.90\right)$. Thus perhaps, the phytochemicals found in whole grains or specifically in wheat bran could be the major factors and main role players responsible for the health benefit of whole grain consumption.

The ORAC values of all samples in this study ranged between 13.5 to $112.5 \mu$ mole TE/g (Trolox equivalent) as shown in Table 3.5. There was significant difference $(P<$ 0.05) among sample values as SRWB had higher ORAC values than HRWB. Bread D had the highest ORAC values in all of the studied bread trials. Therefore, the breads 
which contained ARs had higher ORAC values than the control breads with bread D having significantly higher values than breads B and C.

\subsubsection{Antioxidant activity by \%DPPH}

The $\% \mathrm{DPPH}$ is based on the measurement of the reducing ability of antioxidants and is calculated as a percentage discoloration with a higher percentage suggesting a higher antioxidant capacity. ${ }^{114}$ The \%DPPH scavenging activity of all samples studied during baking is shown in Table 3.4 and ranged from 7.1 to 29.9 . Korycinska et al ${ }^{136}$ reported that the $\% \mathrm{DPPH}$ of whole grain products should be between $7 \%$ to $43 \%$ indicating those values were in agreement with findings from this study. The control breads had the lowest \%DPPH compared to all other bread trials. Bread D contained the highest amount of ARs which also had the highest antioxidant activity compared to the other bread types. The ranking order of \%DPPH inhibition values for WB samples was D $>\mathrm{B}=\mathrm{C}$. The antioxidant activity of WB samples was significantly different from WF $(P$ $<0.05)$. Interestingly, there were no significant differences between the antioxidant activity of WB and SRWB containing residue $(P<0.05)$. This suggests that the presence of other components in bran might affect the antioxidant activity of the above samples. Additionally, the total phenolic components in WB are approximately $3 \mathrm{~g} / 100 \mathrm{~g}^{30}$ which is almost 50 to 60 times higher than its corresponding total ARs (32 to $100 \mathrm{mg} / 100 \mathrm{~g}$ ). ${ }^{46}$ This is likely why the antioxidant activity of WB and SRWB containing residue remained the same. The ORAC assay is a method that can mimic antioxidant activity of phenols in biological systems, making it a more ideal method for antioxidant measurement ${ }^{143}$. Unlike DPPH results, ORAC showed a difference between the antioxidant activity of WB and SRWB containing residue $(P<0.05)$ (Table 3.5). 


\subsection{Conclusion}

In this study, seven bread trials were conducted with the same baking conditions, using two different wheat bran samples to determine whether AR contents and antioxidant capacity changed. The results showed that naturally occurring wheat bran ARs were heat stable during baking. The breads with the highest amount of ARs were also the most elevated and raised trial breads. Furthermore, TPC results showed a positive correlation with $\mathrm{AR}$ content $\left(R^{2}=0.75, P<0.05\right)$. Additional studies are necessary to determine the optimum amount of ARs that should be added in bread or other food model systems. 


\subsection{Connecting Statement to Chapter 4}

In Chapter 3, the stability of natural levels of ARs in baked breads have been investigated by analyzing and comparing total and homologue AR levels using GC-MS. Also, the study aimed to determine how the baking process affects the TPC and the antioxidant activity of ARs by measuring ORAC and DPPH.

In Chapter 4, the characterization of wheat bran ARs by HPLC was studied for different wheat cultivars. The extraction process included both of traditional and supercritical fluid extraction. Also, this chapter gives comparison of antioxidant measurement of wheat bran extracts by different solvent systems.

The work presented in Chapter 4 will be submitted for a publication. 


\section{Chapter 4 Comparison of traditional and supercritical carbon dioxide extractions of ARs, their homolog profile, and bioactivity}

\subsection{Abstract}

Supercritical carbon dioxide ( $\left.\mathrm{SC}-\mathrm{CO}_{2}\right)$ extraction is a non-toxic, non-flammable, low-cost, and environmentally friendly technology which has gained increased interest in the food industry. The $\mathrm{SC}-\mathrm{CO}_{2}$ and traditional organic solvent methods were used to extract wheat bran (WB) ARs from different cultivars. The AR content and profiles were obtained using RP-HPLC and PDA detection. Alkaline hydrolysis and liquid-liquid partitioning steps were carried out to examine ester linked phenolics in WB fractions with soluble free, soluble conjugated and bound forms. Also, the effects of extraction solvents on TPC, DPPH and ORAC were investigated by using three different solvents containing acidified ethanol, $50 \%$ and $100 \%$ acetone. Results showed that the AR content was higher in acetone extracts compared to $\mathrm{SC}-\mathrm{CO}_{2}$ extracts. The most dominant $\mathrm{AR}$ homologue was C21:0, followed by C19:0, C23:0, C17:0 and C25:0. HPLC analysis of phenolics from different WB fraction identified ten phenolic acids of which ferulic acid was the most predominant phenolic acid and mostly found in bound fractions. The same fractions were analyzed for flavonoid contents and six flavonoids were identified with rutin being the predominant one. Two-way ANOVA showed that cultivar, solvent type and interactions of the two, had significantly different effects on TPC, DPPH, and ORAC values $(P<0.05)$. The mean ORAC and DPPH values were highest in the acidified ethanol extracts, whereas the highest TPC values were obtained from the $50 \%$ acetone extracts. 


\subsection{Introduction}

Consumption of cereal grain and the risk of chronic diseases are inversely related. ${ }^{162}$ These health benefits might be attributed to the antioxidant capacity of phenolic compounds in cereals such as ARs and phenolic acids. ${ }^{163}$ Traditionally, ARs have been extracted by different organic solvents including acetone, ethanol, and ethyl acetate. ${ }^{36,79,164}$ The $\mathrm{SC}-\mathrm{CO}_{2}$ extraction technique is very attractive in comparison to traditional organic solvent procedures because of its non-toxic, non-flammable, and environmentally friendly properties. ${ }^{35} \mathrm{SC}-\mathrm{CO}_{2}$ can be used as an extraction solvent to extract ARs, and the efficiency of this extraction method depends on the pressure, temperature, and co-solvents used. ${ }^{66}$ Additionally, it has a faster extraction time than traditional techniques and the $\mathrm{CO}_{2}$ is safe and easy to remove after the extraction is complete. The use of SC- $\mathrm{CO}_{2}$ technology has been well established for the extraction of many different food products, including essential oils. ${ }^{67}$ It has also been used to extract ARs from wheat and rye bran at optimum conditions of $70{ }^{\circ} \mathrm{C}$ and $35 \mathrm{MPa}$ with a flow rate of $25 \mathrm{~g} / \mathrm{min}$ for $4 \mathrm{~h}$ followed by using $10 \%$ ethanol as a co-solvent $/$ modifier. ${ }^{35}$ Due to its non-polar nature, pure $\mathrm{CO}_{2}$ does not solubilize the polar ARs. For this reason, ethanol has been suggested as a co-solvent when using $\mathrm{SC}-\mathrm{CO}_{2}$ for the removal of ARs from wheat and rye bran. Previous studies have found $\mathrm{SC}-\mathrm{CO}_{2}$ to yield $8-80 \%$ more ARs than the traditional extraction using acetone. ${ }^{66}$

Phenolic acids are a predominant group of phenolic antioxidants and can be classified into hydroxybenzoic acids and hydroxycinnamic acids. Ferulic acid is the major phenolic acid of hydroxycinnamic acid derivatives and found esterified or etherified to cereal cell wall components. ${ }^{165}$ Polyphenolic (PP) compounds may exist in 
free or esterified/etherified soluble and insoluble form bound to cell wall constituents like polysaccharides, protein, lignin, cutin or suberin. Insoluble polyphenolic compounds are considered the major contributors to the total antioxidant capacity of cereals. ${ }^{115}$ Most literature data on food, and related biological, nutritional, and epidemiological studies address extractable PP antioxidants. ${ }^{115,166,167}$ However, the analytical methods do not determine the amount of PP remaining in the residues of aqueous organic extraction, named as non-extractable polyphenols, and there is a lack of information in literature on this group of antioxidants. Analytical methodology for cereal PP consists of an extraction with aqueous-organic solvents to obtain soluble polyphenols, followed by a hydrolysis treatment to liberate the bound form. Since acidic hydrolysis might degrade hydroxycinammic and benzoic acids, alkali hydrolysis is the main procedure usually performed in the aqueous-organic extracts for ester bond cleavage. ${ }^{115}$ The major nonextractable PP in DF in the diet are ferulic acid, caffeic acid, hesperidin, naringin, catechin, epicatechin, ellagic acid, gallic acid derivatives, protocatechin, and $p$ hyrdoxybenzoic acid. ${ }^{168}$ Many study reported the influence of different extraction solvents on the content of natural antioxidants in extract. ${ }^{169}$ Solvents such as acetone, methanol, ethanol, propanol and ethyl acetate have been commonly used for the extraction of phenolics from plants. ${ }^{170}$

The objectives of the present work were; I) to investigate feasibility of $\mathrm{SC}-\mathrm{CO}_{2}$ for extracting ARs from wheat bran and compare the $\mathrm{SC}-\mathrm{CO}_{2}$ method with the traditional solvent extraction method of ARs; II) to evaluate the effects of extraction solvents on the antioxidant potential of WB samples by measuring TPC, ORAC and DPPH values; and III) to characterize phenolic fractions as soluble free, soluble conjugated and bound form 
in WB.

\subsection{Materials and methods}

\subsubsection{Materials}

Seven different WB samples were used for this study, including two winter and five spring cultivars (Table 4.1). The winter wheats, hard red wheat bran (HRWB) and soft red wheat bran (SRWB) were obtained from Kraft-Canada. The rest were from Agriculture and Agri-Food Canada (AAFC) breeding programs and were hard spring wheat. The main difference in the samples from the breeding program was the bran color. Win B and Win A were sister lines and they had white bran color as Snowbird, a registered cultivar. The other two, AC Brio and $\mathrm{AC}$ Tahoe were also registered but they had red bran color and usually AC Brio was harder than AC Tahoe. The two genetic lines (Win A and Win B) and AC Brio were from eastern Canada while Snowbird and AC Tahoe was from the Winnipeg program. All chemicals used for extractions and analyses were as described in the section 2.3.2.

Table 4-1 Detailed information about all WB samples used for this study

\begin{tabular}{lllll}
\hline Sample & Source* & Season & Hardness & Colour \\
\hline SRWB & Kraft Canada & winter & soft & red \\
HRWB & Kraft Canada & winter & hard & red \\
AC Brio & AAFC-eastern Canada & spring & hard & red \\
AC Tahoe & AAFC-Winnipeg & spring & hard & red \\
Snowbird & AAFC-Winnipeg & spring & hard & white \\
Win A & AAFC-eastern Canada & spring & hard & white \\
Win B & AAFC-eastern Canada & spring & hard & white \\
\hline
\end{tabular}

* $\mathrm{AAFC}=$ Agriculture and Agri-Food Canada 


\subsubsection{Extraction of ARs}

ARs were extracted using a traditional solvent extraction method, and the green/environmentally friendly $\mathrm{SC}-\mathrm{CO}_{2}$ method. The traditional solvent extraction of ARs has been already described in section 2.3.3.

The $\mathrm{SC}-\mathrm{CO}_{2}$ extraction was performed in a SFE-1000F-2-FMC50 system, from Thar Technology Inc., Pittsburg, USA. It consists of two pumps, one for $\mathrm{CO}_{2}$ and the other for a co-solvent, an automated back pressure regulator (ABPR), an extraction vessel and two collector vessels (Figure 4.1). The $\mathrm{SC}-\mathrm{CO}_{2}$ extraction parameters were chosen based on published studies as summarized in Table 4.2. Ground wheat bran samples (HRWB and SRWB; $150 \mathrm{~g}$ ) were placed in the extraction vessel and $\mathrm{CO}_{2}$ was pumped at $25 \mathrm{~g} / \mathrm{min}$ (flow rate). The pressures of extraction, collector-1 and -2 vessels were set at 350,120 , and 60 bars respectively. The temperatures of extraction, collector- 1 and -2 vessels were set at 70, 45 and $35^{\circ} \mathrm{C}$ respectively. The extractions were performed for $6 \mathrm{~h}$, including $2 \mathrm{~h}$ with $100 \% \mathrm{SC}-\mathrm{CO}_{2}$ and $4 \mathrm{~h}$ with a co-solvent added (10\% ethanol). The extracts obtained with co-solvent were further evaporated and stored at $-20{ }^{\circ} \mathrm{C}$ for further analysis. The AR extraction was made in triplicates. 


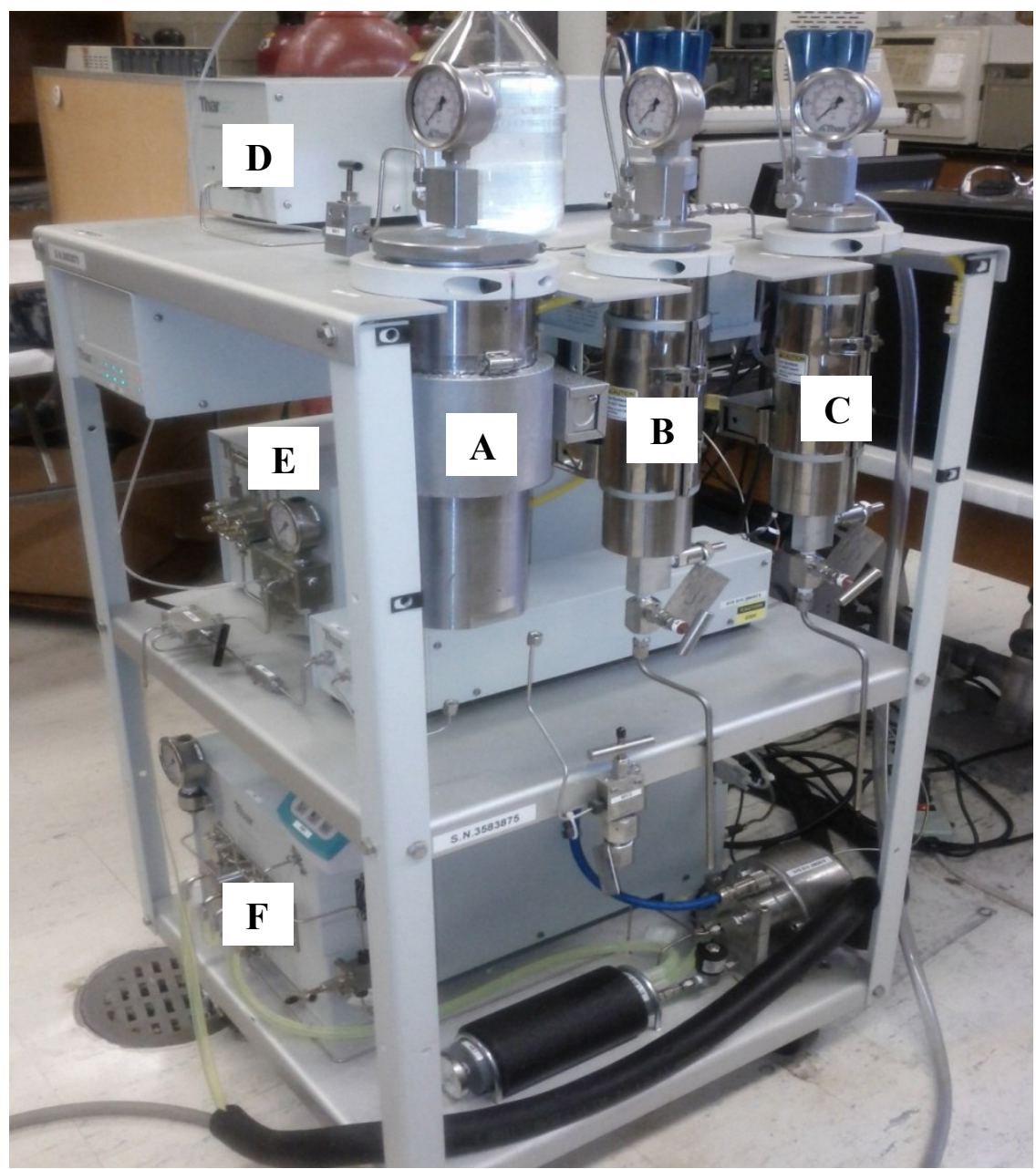

Figure 4-1 SC- $\mathrm{CO}_{2}$ extraction instrument in Dr. Hosseinian's research lab at Carleton University. The letters A, B, C, D, E and F represent the extraction vessel, collector-1, collector-2, automated back pressure regulator (ABPR), co-solvent pump, and $\mathrm{CO}_{2}$ pump, respectively. 
Table 4-2 Comparison of SC-CO2 parameters for ARs extraction of cereal bran

\begin{tabular}{lccc}
\hline Parameters & Landberg et al. $^{68}$ & Francisco et al. $^{66}$ & Athukorala et al. $^{35}$ \\
\hline Sample weight $(\mathrm{g})$ & 20 & 25 & 100 \\
Extraction temperature $\left({ }^{\circ} \mathrm{C}\right)$ & 70 & 40, and 55 & 70 \\
Extraction pressure (bar) & 350 & $80,150,300$ and 350 & 350 \\
Extraction time (h) & 6 & 2 & $2,4,6,8,10$ \\
CO2 flow (g/min) & 5 & 5 & 25 \\
$\%$ Co-solvent (ethanol) & 10 & 10 & 10 \\
\hline
\end{tabular}

\subsubsection{Different solvent extractions for antioxidant activity measurements}

Three different solvents were used to extract phenolic compounds from WB samples and compare solvent effects on the antioxidant activity measurements. The extraction solvents were $100 \%$ acetone, $50 \%$ acetone (in water), and acidified ethanol (95\% ethanol: $1 \mathrm{~N} \mathrm{HCl}(85: 15, \mathrm{v} / \mathrm{v}))$. The first two extractions were performed with a 1:4 ratio $(\mathrm{w} / \mathrm{v})$ for $24 \mathrm{~h},{ }^{140}$ and the last one was performed with a $1: 15$ ratio $(\mathrm{w} / \mathrm{v})$ for $6 \mathrm{~h}$ (section 3.3.4.1). ${ }^{171}$ The resulting supernatant fluid was then stored at $-20^{\circ} \mathrm{C}$ for further analysis (TPC, DPPH and ORAC). All analyses were performed in triplicates.

\subsubsection{Fractional extraction (soluble free, soluble conjugated, and bound phenolics)}

HRWB and SRWB phenolic compounds (phenolic acids and flavonoids) were extracted according to the modified method of Kim et al. ${ }^{172}$ Soluble free, soluble conjugated and bound phenolics were individually extracted in three separate fractions as shown in Figure 4.2. Soluble free phenolics were extracted twice from defatted bran samples by mixing with $80 \%$ methanol $(1 / 10, \mathrm{w} / \mathrm{v}$ ratio) for $1 \mathrm{~h}$ at room temperature $(\mathrm{RT})$ 
and centrifuged at $3900 \mathrm{~g}$ for $15 \mathrm{~min}$. The combined supernatants were driedthen redissolved in water and acidified to $\mathrm{pH} 2$ by $12 \mathrm{M} \mathrm{HCl}$. The soluble free phenolics fraction was extracted twice by ethyl ether $(1 / 1, \mathrm{v} / \mathrm{v})$, dried then re-dissolved in methanol for HPLC analysis. The second extract fraction was assigned as soluble conjugated phenolics. The extraction was started the same way for free fraction however after drying the supernatant, samples were re-dissolved in $2 \mathrm{M} \mathrm{NaOH}$ and agitated at $\mathrm{RT}$ for $4 \mathrm{~h}$ to hydrolyze and release the conjugated phenolics. After hydrolysis, the acidification and ethyl acetate extraction steps were followed the same as in the extraction of soluble free phenolics steps. Finally, the bound phenolics fraction was obtained by re-dissolving the dried residue from the first extraction (crude phenolics extraction) in $2 \mathrm{M} \mathrm{NaOH} \mathrm{(1:40,}$ $\mathrm{w} / \mathrm{v}$ ) and agitating for 4 hour at RT followed by acidification and ethyl acetate extraction. The dried extracts were re-dissolved in $\mathrm{MeOH}$ for the determination of phenolic acid and flavanoid content. Extracts were filtered with a $0.45 \mu \mathrm{m}$ PTFE filter before HPLC analysis. 


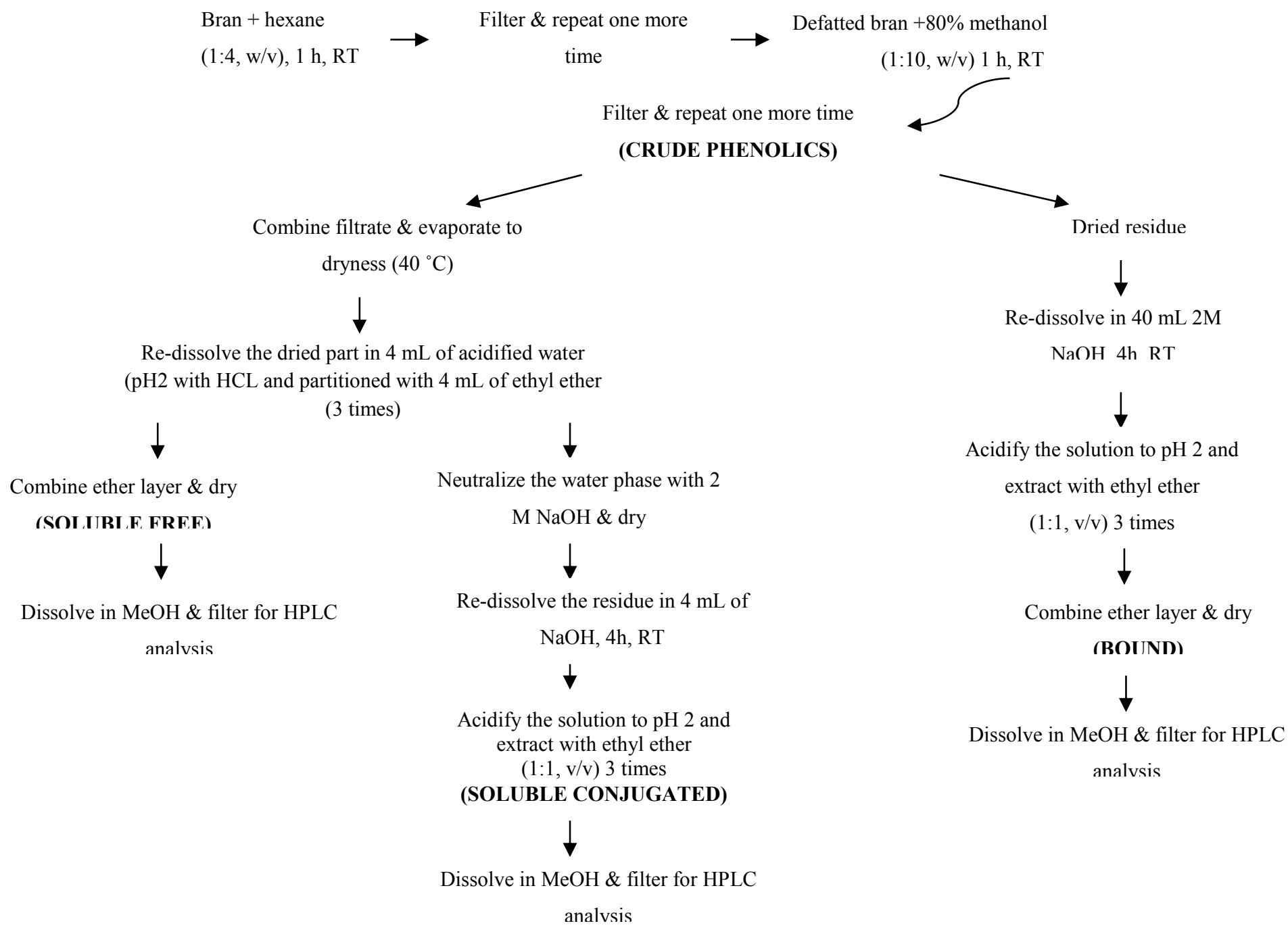

Figure 4-2 Soluble free, soluble conjugated and bound phenolic acid extractions procedures (modified from Kim et.al). ${ }^{172}$ 


\subsubsection{HPLC analysis}

Both traditional and $\mathrm{SC}-\mathrm{CO}_{2}$ crude extracts of ARs, and fractional extracts of phenolics were analyzed via a reverse phase (RP)-HPLC on the Alliance ${ }^{\circledR}$ HPLC system e2695 Separation Module with the 2998 Photodiode Array Dedector (PDA) from Waters (Mildford, Massacchusetts, USA). The software used was Empower 3. The details about HPLC run parameters are explained in the following sections.

\subsubsection{HPLC analysis of ARs}

The standards of ARs as a mixture of AR homologues C15:0-C25:0 and all sample crude extracts of ARs were prepared in methanol. A gradient program followed to separate the different homologues at a flow rate $1 \mathrm{~mL} / \mathrm{min}$ and UV detection at $280 \mathrm{~nm}$ with a RP column (Synergi-Max-RP, $250 \times 4.6 \mathrm{~mm}, 5 \mu \mathrm{m}$ ) a temperature set at $35{ }^{\circ} \mathrm{C}$. Solvent A was $1 \%$ acetic acid in $\mathrm{MeOH}$ and solvent B was $2 \%$ acetic acid in water. The gradient program was summarized in Table 4.3.

Table 4-3 The gradient program for HPLC analysis of ARs

\begin{tabular}{lllll}
\hline & Time & Flow & $\% \mathrm{~A}$ & \%B \\
\hline \hline 1 & 00.00 & 1.00 & 90.0 & 10.0 \\
2 & 10.00 & 1.00 & 100.0 & 00.0 \\
3 & 25.00 & 1.00 & 100.0 & 00.0 \\
4 & 35.00 & 1.00 & 90.0 & 10.0 \\
5 & 50.00 & 1.00 & 90.0 & 10.0 \\
\hline
\end{tabular}




\subsubsection{HPLC analysis of phenolics}

Phenolic acid and flavonoid standards, samples, and fractional extracts were prepared in methanol. A gradient program was developed to separate phenolic acids and flavonoids in a single run with a Synergi-Max-RP column, at $35{ }^{\circ} \mathrm{C}$. Solvent A was $0.01 \%$ formic acid/water and solvent $\mathrm{B}$ was $100 \%$ acetonitrile at a flow rate of 1.0 $\mathrm{mL} / \mathrm{min}$ and a gradient program as shown in Table 4.4. The wavelengths used for qualitative and quantitative analysis for phenolic acid was $280 \mathrm{~nm}$, and for flavonoids was 280 and $320 \mathrm{~nm}$. Also, spectral data at 200-700 $\mathrm{nm}$ were recorded for all sample and standard. For all 11 phenolic acid and 10 flavonoid standards (Table 4.8), five different concentrations were prepared as a mixture of standards. Standard curves of each phenolic acid and flavonoid mixture were plotted using the corresponding peak areas. For all standard curves, the regression value ranged between 0.9956 and 1.0000. All samples were analyzed in duplicate and concentration of each phenolic acid and flavonoids was calculated using a standard curve equation and expressed in $\mathrm{mg} / \mathrm{g}$ of wheat bran samples.

Table 4-4 The gradient program for HPLC analysis of phenolic acid and flavonoids

\begin{tabular}{lcccc}
\hline & Time & Flow & $\% \mathrm{~A}$ & $\% \mathrm{~B}$ \\
\hline 1 & 00.00 & 1.00 & 90.0 & 10.0 \\
2 & 35.00 & 1.00 & 50.0 & 50.0 \\
3 & 40.00 & 1.00 & 90.0 & 10.0 \\
4 & 50.00 & 1.00 & 90.0 & 10.0 \\
\hline
\end{tabular}




\subsubsection{Antioxidant activity measurements}

All wheat bran extracts from three different solvents (section 4.3.3) were analyzed for measuring TPC, DPPH, and ORAC values. The TPC, DPPH and ORAC measurements were performed by following the methods described in the sections 2.3 .5 , 2.3.6, and 2.3.7, respectively.

\subsubsection{Statistical analysis}

All statistical analyses were performed using SAS version 9.1 (SAS Institute Inc., Cary, NC, USA). Analysis of variance (ANOVA) was used to determine if there was a significant difference $(P<0.05)$ among triplicates of total and homologue of ARs for both traditional and $\mathrm{SC}-\mathrm{CO}_{2}$ extractions. Significant treatments were further analyzed with the Duncan's multiple range test $(P<0.05)$. Also a two-way analysis of ANOVA was performed to see if there was significant difference $(P<0.05)$ between ORAC, DPPH and TPC data sets of different solvents. 


\subsection{Results and discussion}

\subsubsection{AR content and homolog composition by traditional method}

The total ARs and homologue contents $(\mathrm{mg} / 100 \mathrm{~g})$ of different wheat cultivars extracted by acetone are presented in Table 4.5. The saturated ARs were confirmed in comparison by the retention times of AR standards $(\mathrm{C} 15: 0-\mathrm{C} 25: 0)$ as shown in the Figure 4.3.
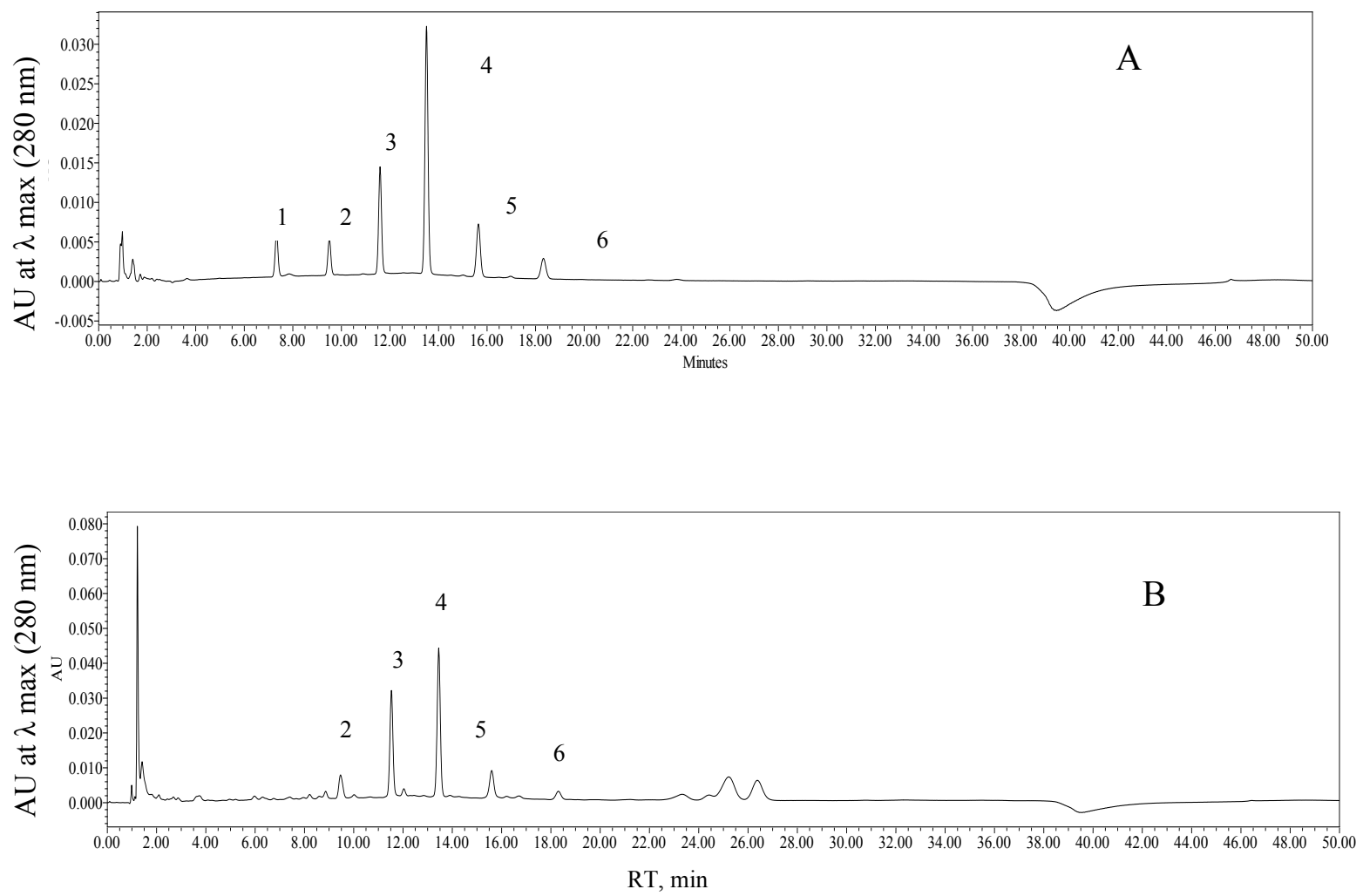

Figure 4-3 HPLC chromatograms of ARs standard mixture (A) and HRWB sample (B).The numbers represent each ARs homologue including C15:0 (1), C17:0 (2), C19:0 (3), C21:0 (4), C23:0 (5), and C25:0 (6) 
Table 4-5 Total ARs and homolog contents $(\mathrm{mg} / 100 \mathrm{~g}$ WB) of different wheat cultivars extracted by acetone

\begin{tabular}{llllllll}
\hline Composition & HRWB & SRWB & AC Brio & AC Tahoe & Snowbird & Win A & Win B \\
\hline Total- ARs & $447.8 \pm 10.1^{\mathrm{a}}$ & $305.8 \pm 8.2^{\mathrm{b}}$ & $285.0 \pm 12.3^{\mathrm{b}}$ & $258 \pm 11.8^{\mathrm{c}}$ & $245.9 \pm 9.0^{\mathrm{c}}$ & $192.3 \pm 7.7^{\mathrm{d}}$ & $144.0 \pm 2.0^{\mathrm{e}}$ \\
$\mathrm{C} 15: 0$ & nd & nd & $2.3 \pm 0.1^{\mathrm{b}}$ & nd & $10.7 \pm 0.4^{\mathrm{a}}$ & nd & nd \\
C17:0 & $44.0 \pm 0.4^{\mathrm{a}}$ & $19.5 \pm 1.5^{\mathrm{ed}}$ & $34.3 \pm 1.9^{\mathrm{b}}$ & $28.8 \pm 2.8^{\mathrm{cb}}$ & $24.2 \pm 0.1^{\mathrm{cd}}$ & $12.9 \pm 0.9^{\mathrm{ef}}$ & $10.7 \pm 3.8^{\mathrm{f}}$ \\
$\mathrm{C} 19: 0$ & $138.1 \pm 5.1^{\mathrm{a}}$ & $92.9 \pm 6.6^{\mathrm{b}}$ & $80.5 \pm 1.0^{\mathrm{cb}}$ & $76.0 \pm 4.5^{\mathrm{c}}$ & $79.2 \pm 4.5^{\mathrm{cb}}$ & $59.9 \pm 6.4^{\mathrm{d}}$ & $44.6 \pm 3.2^{\mathrm{e}}$ \\
$\mathrm{C} 21: 0$ & $193.6 \pm 2.5^{\mathrm{a}}$ & $136.4 \pm 4.1^{\mathrm{b}}$ & $113.5 \pm 6.3^{\mathrm{c}}$ & $92.2 \pm 6.0^{\mathrm{d}}$ & $85.6 \pm 5.9^{\mathrm{ed}}$ & $80.1 \pm 3.5^{\mathrm{e}}$ & $58.9 \pm 4.4^{\mathrm{f}}$ \\
$\mathrm{C} 23: 0$ & $53.0 \pm 1.2^{\mathrm{a}}$ & $44.6 \pm 1.7^{\mathrm{ba}}$ & $37.1 \pm 2.2^{\mathrm{bc}}$ & $13.8 \pm 4.9^{\mathrm{d}}$ & $26.5 \pm 5.0^{\mathrm{dc}}$ & $28.4 \pm 3.4^{\mathrm{dc}}$ & $23.5 \pm 1.7^{\mathrm{dc}}$ \\
$\mathrm{C} 25: 0$ & $19.1 \pm 0.9^{\mathrm{a}}$ & $12.9 \pm 2.7^{\mathrm{b}}$ & $17.1 \pm 2.9^{\mathrm{b}}$ & $47.4 \pm 4.0^{\mathrm{a}}$ & $19.8 \pm 0.3^{\mathrm{b}}$ & $11.0 \pm 1.9^{\mathrm{b}}$ & $6.3 \pm 0.1^{\mathrm{b}}$ \\
\hline
\end{tabular}

${ }^{*}$ Values are means of triplicates \pm standard deviations (SD). Different letters in rows for each characteristic are significantly different $(P<0.05)$ in Duncan's multiple range tests, $\mathrm{nd}=$ not detected 
There was a significant difference $(P<0.05)$ in the total AR and homolog contents of all the samples extracted by acetone. The order of total AR content from highest to lowest of all WB samples was recorded as:

$$
\text { HRWB }>\text { SRWB }=\text { Brio }>\text { Tahoe }=\text { Snowbird }>\text { Win } A>\text { Win B }
$$

Due to unavailability of standards, unsaturated ARs were identified according to their retention time ranges, eluting prior to or after each saturated AR homologue (C15C25). ${ }^{173}$ Although the total AR content was different for each WB sample, the relative saturated (80.4\%) and unsaturated ARs (19.6\%) for HRWB remained in close range and our findings were in agreement with values reported in literature. ${ }^{140,173}$ The ANOVA revealed that for each cultivar, the mean saturated AR content (74.4\%) in winter wheats was higher than that of spring wheats $(60.5 \%)$. Genetic factors, climate, season, grain filling period, cereal type and soil conditions have been reported to affect AR content. ${ }^{46}$

It has been suggested that ARs may have protective antioxidant effects in biological membranes and also have stimulant or inhibitory effects on some metabolic enzymes. The length of the alkyl chain affects the extent of inhibition, with C17:0 and C19:1 being the most effective inhibitors. ${ }^{94}$ Moreover, resorcinolic lipids from cereal bran have shown inhibitory activity of soybean lipoxygenases ${ }^{91}$ and prevent triglyceride accumulation in cultured 3T3-L1 cells, indicating that they may inhibit triglyceride synthesis in vivo. ${ }^{93}$

The order of individual AR homologue content from highest to lowest was C21:0 $>\mathrm{C} 19: 0>\mathrm{C} 23: 0>\mathrm{C} 17: 0>\mathrm{C} 25: 0$. In this study, the relative AR homologue composition for all WB samples examined was determined to be in the ranges of $0-4.5 \%$ for $\mathrm{C} 15: 0$, 6.2-11.9\% for $\mathrm{C} 17: 0,28.4-32.1 \%$ for $\mathrm{C} 19: 0,31.3-44.6 \%$ for $\mathrm{C} 21: 0,5.4-14.4 \%$ for $\mathrm{C} 23: 0$, 
and 4.2-10.5\% for C25:0. The most abundant AR homologues were C21:0, and C19:0 in WB samples which is in agreement with other reports; Knodler et al, ${ }^{145}$ and Gunenc et al. ${ }^{173}$ The C15:0 homologue was only detected in Brio $(2.3 \pm 0.1 \mathrm{mg} / 100 \mathrm{~g})$ and Snowbird $(10.7 \pm 0.4 \mathrm{mg} / 100 \mathrm{~g}) \mathrm{WB}$ samples and that was expected due to the fact that $\mathrm{C} 15: 0$ does not generally occur in WB compared to the other homologues $(\mathrm{C} 17: 0-\mathrm{C} 25: 0)$. The ratio of $\mathrm{C} 17: 0$ to $\mathrm{C} 21: 0$ for durum wheat, common wheat, triticale, and rye are approximately $0.01,0.1,0.2$ and 1.0 respectively. ${ }^{46}$ For this study, the ratio of $\mathrm{C} 17: 0 / \mathrm{C} 21: 0$ ranges from 0.07 to 0.30 and these ratios are suggested to be an index for determining the origin of a cereal product. Our study showed that these ratios could be used to differentiate common wheat cultivars.

\subsubsection{AR content and homolog composition by $\mathrm{SC}-\mathrm{CO}_{2}$ method}

Both HRWB and SRWB were extracted with $\mathrm{SC}-\mathrm{CO}_{2}$. The total AR and homolog contents (mg/100 g WB) were presented in Table 4.6. The total AR content of HRWB and SRWB was calculated as 74.8, and $64.2 \mathrm{mg} / 100 \mathrm{~g}$ respectively, findings which were in agreement with that of Athukorala et al. ${ }^{35}$ In their study, total AR contents for triticale Ultima, triticale Pronghorn, and wheat were recorded as 70, 36, and $68 \mathrm{mg} / 100 \mathrm{~g}$ respectively; similar conditions were used in this study $\left(70{ }^{\circ} \mathrm{C}, 25 \mathrm{~g} / \mathrm{min}, 350 \mathrm{bar}\right)$.

When both extraction methods were compared, the $\mathrm{SC}-\mathrm{CO}_{2}$ extracts had $35 \%$ (HRWB) and 38\% (SRWB) lower total yields than acetone extracts. At the same time, the total AR content in $\mathrm{SC}-\mathrm{CO}_{2}$ extracts was $79 \%$ (HRWB) and $83 \%$ (SRWB) less than that of acetone extracts. In the traditional method, WB samples were extracted with $100 \%$ acetone for $24 \mathrm{~h}$ whereas in $\mathrm{SC}-\mathrm{CO}_{2}$ extraction, only $10 \%$ ethanol was used as a cosolvent for $4 \mathrm{~h}$. When the extraction solvents and conditions were taken into 
consideration, differences in AR content can be better explained. Also, HPLC analysis of the first extraction step $\left(100 \% \mathrm{CO}_{2}\right)$ confirmed that none of the ARs homologues were detected, indicating $100 \% \mathrm{SC}-\mathrm{CO}_{2}$ solvent is not appropriate to extract polar lipids such as ARs. Fransciso et al.(2005) also reported that none of the AR homologues were detected in the extract when $100 \% \mathrm{CO}_{2}$ was used under the same extraction conditions $\left(350 \mathrm{bar}, 55-70{ }^{\circ} \mathrm{C}\right){ }^{66}$ Therefore in our study, the first extraction step with $100 \% \mathrm{SC}-\mathrm{CO} 2$ was performed to remove the free-ARs lipid fraction. The combination of a polar cosolvent with a non-polar fluid $\left(\mathrm{CO}_{2}\right)$ increased the extractability of ARs. In the second extraction step of $\mathrm{SC}-\mathrm{CO}_{2}$ which incorporated a co-solvent, the sum of $1^{\text {st }}$ and $2^{\text {nd }}$ collectors had 61\% (HRWB) and 55\% (SRWB) fewer yields compared to the acetone extract.

In earlier studies, for acetone extractions of ARs from cereal bran, the most abundant AR-homologues have been reported to be $\mathrm{C} 17: 0-\mathrm{C} 21: 0$ (up to $85 \%$, w/w). ${ }^{66,90}$ However, in this study these homologues accounted for only $70 \%$ of ARs. Several factors such as a difference in sample source, sample nature, extraction method and protocol might suggest some reasons for these variations. ${ }^{66}$ Although total yields and solvents used were different in both extraction methods, the homologue distribution pattern remained similar. The order of individual AR homologue contents from the highest to lowest percentage matched that of acetone extracts, $\mathrm{C} 21: 0>\mathrm{C} 19: 0>\mathrm{C} 23: 0>\mathrm{C} 17: 0>$ C25:0.For SC- $\mathrm{CO}_{2}$ extracts, the relative AR homologue content for both WB (HRWB and SRWB) samples were determined to be $5.3-7.8 \%$ for $\mathrm{C} 17: 0,30.7-43.8 \%$ for $\mathrm{C} 19: 0$, 50.7-53.1\% for $\mathrm{C} 21: 0,8-11 \%$ for $\mathrm{C} 23: 0$, and $2.7-3.1 \%$ for $\mathrm{C} 25: 0$. The dominant AR homologues were $\mathrm{C} 21: 0(52 \%)$ and $\mathrm{C} 19: 0(37 \%)$, results that are in agreement with 
Rebolleda et al. ${ }^{174}$ Several factors such as genetic factors, climate, season, grain filling and soil conditions might affect the AR content, ${ }^{35}$ similar $\% \mathrm{AR}$ profiles were also found by Landberg et al.(2007) in the extraction of wheat bran using ethyl acetate and SC$\mathrm{CO}_{2}{ }^{68}$ Although similar AR profiles were obtained by both extraction methods, it must be emphasized that the highest $\mathrm{AR} /$ extract ratio was obtained when using a polar organic solvents such as acetone. The ratios of HRWB and SRWB for total \%AR extracted with acetone were $15.6 \%$ and $10.9 \%$, respectively. These ratios for $\mathrm{SC}-\mathrm{CO}_{2}$ extraction for HRWB and SRWB were $6.6 \%$ and $5.1 \%$ respectively. These findings are in agreement with literature. ${ }^{68,174}$ Even though the higher AR yield obtained by traditional extraction method compared to $\mathrm{SC}-\mathrm{CO}_{2}$, solvent extractions present some disadvantages like long extraction times, toxic waste generation and a more lengthy final purification procedure. $^{66}$ 
Table 4-6 Total ARs and homolog contents (mg/100g WB) of HRWB and SRWB samples extracted by $\mathrm{SC}_{-} \mathrm{CO}_{2}$

\begin{tabular}{|c|c|c|c|c|c|c|c|c|}
\hline Sample & Collector & C 17:0 & $\mathrm{C} 19: 0$ & $\mathrm{C} 21: 0$ & $\mathrm{C} 23: 0$ & $\mathrm{C} 25: 0$ & ARs & $\begin{array}{l}\text { Total ARs } \\
\left(1^{\text {st }}+2^{\text {nd }}\right)\end{array}$ \\
\hline \multirow[t]{2}{*}{ HRWB } & $1^{\text {st }}$ & $1.1 \pm 0.5^{\mathrm{a}}$ & $5.2 \pm 2.9^{b}$ & $8.1 \pm 4.1^{\mathrm{c}}$ & $1.5 \pm 0.7^{\mathrm{b}}$ & $1.1 \pm 0.8^{\mathrm{a}}$ & $17.0 \pm 9.1^{b}$ & $74.8 \pm 17.3^{\mathrm{a}}$ \\
\hline & $2^{\text {nd }}$ & $2.6 \pm 0.8^{\mathrm{a}}$ & $18.7 \pm 2.9^{\mathrm{a}}$ & $29.9 \pm 2.5^{\mathrm{a}}$ & $4.9 \pm 0.5^{\mathrm{a}}$ & $1.7 \pm 0.2^{\mathrm{a}}$ & $57.8 \pm 7.8^{\mathrm{a}}$ & \\
\hline \multirow[t]{2}{*}{ SRWB } & $1^{\text {st }}$ & $1.9 \pm 0.3^{\mathrm{a}}$ & $7.5 \pm 0.1^{b}$ & $13.8 \pm 0.1^{\mathrm{cb}}$ & $2.6 \pm 0.6^{\mathrm{ba}}$ & $0.6 \pm 0.5^{\mathrm{a}}$ & $26.4 \pm 1.8^{b}$ & $64.2 \pm 12.8^{\mathrm{a}}$ \\
\hline & $2^{\text {nd }}$ & $2.7 \pm 1.6^{\mathrm{a}}$ & $11.2 \pm 3.3^{b a}$ & $19.6 \pm 4.3^{b}$ & $3.5 \pm 1.2^{\mathrm{ba}}$ & $0.8 \pm 0.3^{\mathrm{a}}$ & $37.8 \pm 11.0^{\mathrm{ba}}$ & \\
\hline
\end{tabular}

${ }^{*}$ Values are means of triplicates \pm standard deviations (SD). Different letters in columns for each characteristic are significantly different $(P<0.05)$ in Duncan's multiple range tests. 


\subsubsection{Phenolic acids and flavonoids content and composition analysis of fractional extraction of phenolics}

In the present study, alkaline hydrolysis and liquid-liquid partitioning steps were carried out to extract ester linked phenolics from both WB (HRWB \& SRWB) and their fractions, assigned as soluble free, soluble conjugated and bound forms. The separation of phenolic acids in the WB samples and its alkaline extracts were carried out on a RPHPLC. The phenolic acid content and composition (mg/g of sample) of HRWB and SRWB fractions including soluble free (A), soluble conjugated (B), and bound fractions (C) are shown in Table 4.7. 
Table 4-7 Phenolic acid (PA) content (mg/g WB) and composition of HRWB and SRWB fractions including soluble free (A), soluble conjugated $(\mathrm{B})$, and bound $(\mathrm{C})$

\begin{tabular}{|c|c|c|c|c|c|c|c|c|c|c|c|c|}
\hline Sample & Fraction & $\begin{array}{l}\text { Total } \\
\text { (PA) }\end{array}$ & $1^{*}$ & 2 & 3 & 4 & 5 & 6 & 7 & 8 & 9 & 10 \\
\hline \multirow[t]{3}{*}{ HRWB } & A & $0.20 \pm 0.14$ & nd & nd & $0.02 \pm 0.01$ & $0.15 \pm 0.11$ & nd & nd & nd & $0.02 \pm 0.01$ & nd & $0.01 \pm 0.01$ \\
\hline & $\mathrm{B}$ & $0.44 \pm 0.04$ & $0.08 \pm 0.00$ & nd & $0.05 \pm 0.04$ & $0.22 \pm 0.16$ & $0.10 \pm 0.09$ & nd & $0.03 \pm 0.02$ & $0.05 \pm 0.04$ & $0.02 \pm 0.01$ & $0.05 \pm 0.05$ \\
\hline & $\mathrm{C}$ & $3.01 \pm 0.57$ & $0.60 \pm 0.75$ & nd & $0.07 \pm 0.05$ & $0.40 \pm 0.28$ & nd & $0.06 \pm 0.04$ & $0.23 \pm 0.26$ & $0.33 \pm 0.29$ & $0.08 \pm 0.02$ & $1.52 \pm 1.49$ \\
\hline \multirow[t]{3}{*}{ SRWB } & A & $0.10 \pm 0.04$ & nd & nd & $0.01 \pm 0.01$ & $0.07 \pm 0.05$ & nd & nd & nd & nd & nd & nd \\
\hline & $\mathrm{B}$ & $1.82 \pm 2.02$ & $0.47 \pm 0.58$ & $0.27 \pm 0.35$ & $0.27 \pm 0.07$ & $0.18 \pm 0.13$ & $0.03 \pm 0.02$ & $0.19 \pm 0.13$ & $0.13 \pm 0.17$ & $0.16 \pm 0.21$ & $0.02 \pm 0.02$ & $0.30 \pm 0.38$ \\
\hline & $\mathrm{C}$ & $2.09 \pm 1.66$ & $0.44 \pm 0.31$ & nd & $0.07 \pm 0.05$ & $0.40 \pm 0.28$ & nd & 0.03 & $0.04 \pm 0.03$ & $0.15 \pm 0.03$ & nd & $1.43 \pm 1.66$ \\
\hline
\end{tabular}

$* 1=$ gallic, $2=$ proto-catechuic, $3=$ p-OH-benzoic, $4=$ chlorogenic, $5=$ caffeic, $6=$ vanillic, $7=$ syringic, $8=$ p-coumaric, $9=$ sinapic, $10=$ ferulic, nd $=$ not detected

Values are means of triplicates \pm standard deviations (SD) 
Fraction C (bound form) for both WB samples had the highest total phenolic acid content $(52 \%$ for SRWB and $82 \%$ for HRWB) compared to the other fractions, A (soluble free) and B (soluble conjugated). Total phenolic acid contents ranged from 12$45 \%$ for B fractions, and 3-6\% for A fractions. These findings were expected since most of the phenolic acids were found in bound form. ${ }^{30}$

With HPLC analysis, a total of ten phenolic acid peaks identified in the fractions (A, B and C) were respectively assigned as gallic, proto-catechmic, p-OH-benzoic, chlorgenic, vanillic, syringic, p-coumaric, sinapic, and ferulic acids with their retention times compared with standards (Figure 4.4). There were no significant differences $(P<$ 0.05) in the content of each phenolic acid per fraction. The WB samples tested were winter red wheats. Further studies are needed to compare phenolic acid compositions indifferent cultivars and regions. Comparing the present study with published data was difficult because of differences in wheat cultivars used, pre-treatment of the flour or bran, and solvents used to extract free phenolics, ${ }^{116}$ as well as differences in acidic and/or alkaline hydrolysis. ${ }^{175}$

Ferulic acid (FA) was detected as the predominant phenolic acid and mostly in bound form in C fractions (82-96\%).Mean FA content ranged from $0.842-1.523 \mathrm{mg} / \mathrm{g}$. High levels of FA in the $\mathrm{C}$ fractions showed that esterified FA was concentrated in those fractions and this finding is in agreement with literature. ${ }^{176}$ 

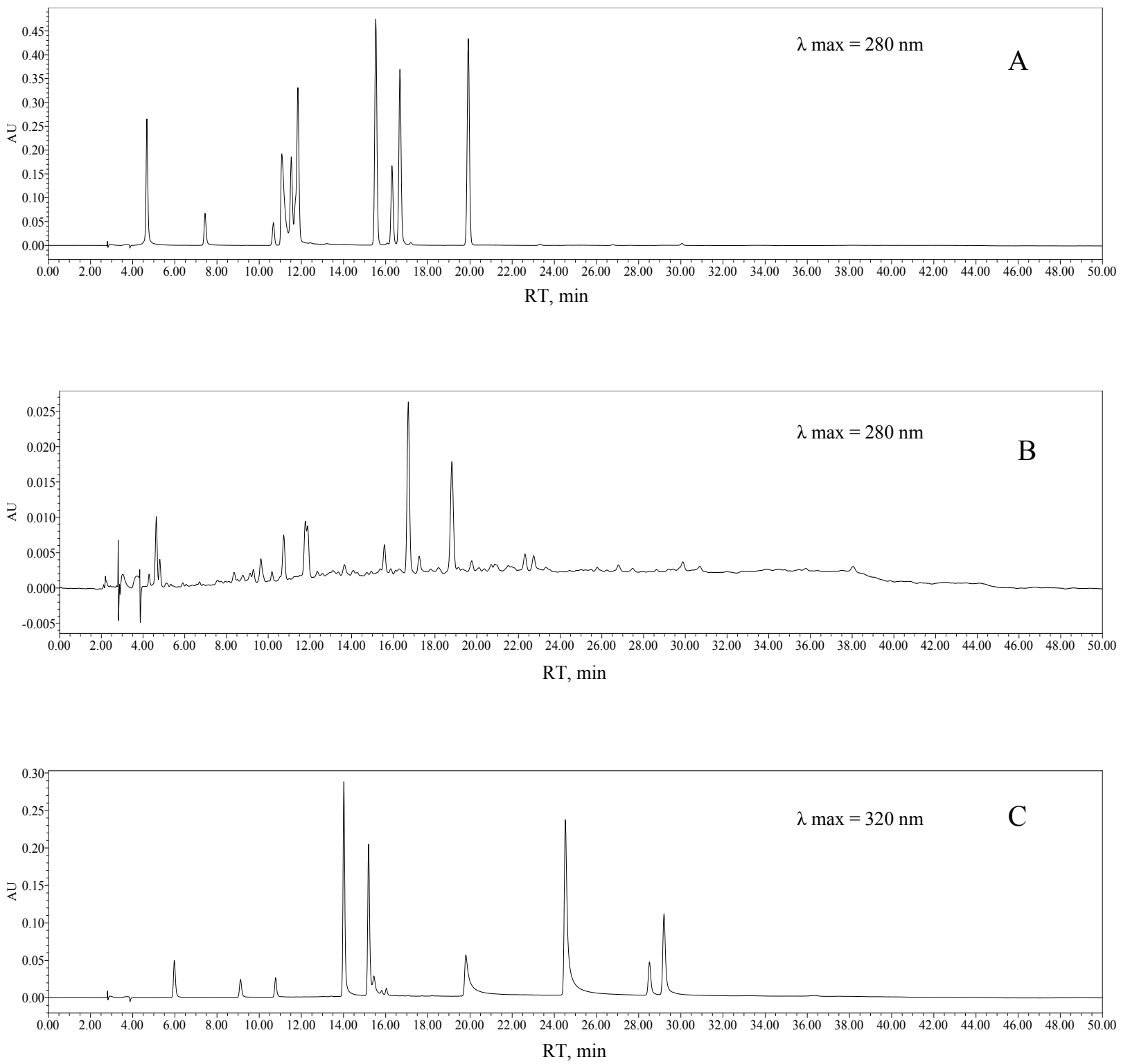

Figure 4-4 HPLC chromatograms of phenolic acid standard (A), SRWB soluble conjugated phenolic acids (B), flavonoid standards (C) (Look at Table 4.8 for each standard and corresponding retention times) 
Table 4-8 Phenolic acid and flavonoids standards used for HPLC analysis and their retention times $(\mathrm{RT})$ in minute

\begin{tabular}{ll|lc}
\hline Phenolic acids & RT & Flavonoids & RT \\
\hline \hline Gallic acid & 4.73 & Pyrogallol & 5.97 \\
Proto-catechic acid & 7.71 & Catechin & 9.13 \\
$p$-OH-benzoic acid & 10.76 & Epicatechin & 10.76 \\
Chlorogenic acid & 11.10 & Rutin & 14.00 \\
Caffeic acid & 11.56 & Quercetin 3 beta glucoside & 15.17 \\
Vanillic acid & 11.76 & Epicatechin gallate & 15.40 \\
Syringic acid & 11.89 & Myricetin & 19.71 \\
$p$-coumaric acid & 15.60 & Quercetin & 24.44 \\
Sinapic acid & 16.38 & Apigenin & 28.45 \\
Ferulic acid & 16.75 & Kaempherol & 29.14 \\
$o$-coumaric & 19.99 & & \\
\hline
\end{tabular}

The total phenolic content obtained in this study $(3.703-4.036 \mathrm{mg} / \mathrm{g})$ is in the range reported by Verme et al. (2009), whereby similar hydrolysis conditions were used. Their total phenolic acid content ranged from 2.784 for AC Andrew wheat bran to $4.043 \mathrm{mg} / \mathrm{g}$ for Kathew wheat bran. ${ }^{175}$

Moreover, the same fractions were analyzed for flavonoid content by HPLC and the results are presented in Table 4.9. This Table shows flavonoid content and composition (mg/g of sample) of HRWB and SRWB fractions containing the three fractions A, B and C. Six major flavonoid peaks in the fractions were pyrogallol, catechin, epicatechin, rutin, quercetin-3-beta glucoside and epicatechin gallate by comparison of their retention times with standards. The phytochemical profiles, 
especially the flavonoid profiles of wheat, have only been studied in recent years. ${ }^{116}$ Moreover, little is known about the inherent differences in phytochemical profiles. Those biologically active components have not received as much attention as the phytochemicals in fruits and vegetables although the increased consumption of whole grains and whole grain products has been linked to a reduced risk of chronic disease development. ${ }^{163}$ Our findings for flavonoid content were also comparable to that of Feng et al. ${ }^{177}$ They characterized flavonoids in the bran of four classes of wheat, including three hard red winter classes and one durum class; the mean flavonoid content found in this study was $0.30 \mathrm{mg} / \mathrm{g}$ compared to that of our findings of $0.64 \mathrm{mg} / \mathrm{g}$. 
Table 4-9 Flavonoids contents (FC, $\mathrm{mg} / \mathrm{g}$ of $\mathrm{WB}$ ) and composition of HRWB and SRWB fractionals including soluble free (A), soluble conjugated (B), and bound (C)

\begin{tabular}{llrrrrrrr}
\hline Sample & Fraction & Total (FC) & $1^{*}$ & 2 & 3 & 4 & 5 \\
\hline HRWB & A & $0.10 \pm 0.02$ & $0.07 \pm 0.01$ & nd & $0.01 \pm 0.00$ & $0.02 \pm 0.02$ & nd \\
& B & $0.14 \pm 0.10$ & nd & $0.05 \pm 0.04$ & nd & nd & $0.10 \pm 0.05$ & nd \\
& C & $0.26 \pm 0.19$ & $0.11 \pm 0.02$ & nd & nd & $0.30 \pm 0.22$ & nd & nd \\
SRWB & A & $0.11 \pm 0.06$ & $0.04 \pm 0.03$ & $0.01 \pm 0.00$ & $0.14 \pm 0.01$ & $0.01 \pm 0.01$ & nd & nd \\
& B & $0.38 \pm 0.28$ & $0.02 \pm 0.02$ & $0.23 \pm 0.17$ & nd & $0.25 \pm 0.33$ & nd & nd \\
& C & $0.27 \pm 0.03$ & $0.05 \pm 0.04$ & $0.10 \pm 0.07$ & nd & $0.10 \pm 0.07$ & nd & $0.15 \pm 0.01$ \\
\hline
\end{tabular}

*1 = pyrogallol, 2 = catechin, $3=$ epicatechin, 4 = rutin, $5=$ quercetin-3-beta glucoside, $6=$ epicatechin gallate, nd $=$ not detected

Values are means of triplicates \pm standard deviations (SD) 


\subsubsection{Antioxidant activity}

Wheat contains many classes of antioxidant compounds including phenolic acids, carotenoids, tocopherols, and tocotrienols. Most of these compounds such as phenolic acids are present in free, esterified or glycosylated, and additionally as complexes with

proteins and other plant materials. ${ }^{115,116,149}$ Also, the concentration of each antioxidant compound in wheat grains depends on the genotype, growing conditions, and interactions between genotype and environment. For these aforementioned reasons, many extraction conditions have been used by different research groups making inter-laboratory comparisons of wheat antioxidant assays difficult. ${ }^{112}$

The purpose of this section was to compare three different solvent systems for extracting antioxidants from different wheat cultivars and their influence on antioxidant activity estimation by comparing TPC, ORAC and DPPH assays of those crude WB extracts.

The WB samples were extracted with acidified ethanol, 50\% acetone and 100\% acetone. Solvent effects on TPC, ORAC and DPPH were investigated. The results are presented below.

\subsubsection{TPC}

Solvent effects on TPC measurement was investigated using five hard spring wheats (Brio, Tahoe, Snowbird, Win A and Win B) as well as one hard (HRWB) and one soft (SRWB) winter wheat (Table 4.1). Extraction solvent types had significant effects $(P$ $<0.05)$ on TPC as seen in Table 4.10. 
Regardless of wheat variety, the WB samples extracted with $50 \%$ acetone displayed the highest TPC values followed by acidified ethanol and $100 \%$ acetone extracted samples. Use of 50\% acetone for winter wheat (HRWB and SRWB) exhibited significantly higher TPC values than spring wheat, suggesting that $50 \%$ acetone might be a preferred solvent for extracting WB rather than acidified ethanol or $100 \%$ acetone.

Table 4-10 TPC (mg FAE/g WB) values of different wheat cultivars extracted by three different solvent systems

\begin{tabular}{llll}
\hline Sample & Acidified ethanol & $50 \%$ acetone & $100 \%$ acetone \\
\hline HRWB & $9.8 \pm 0.7^{\mathrm{c}}$ & $26.3 \pm 0.7^{\mathrm{a}}$ & $14.6 \pm 1.2^{\mathrm{a}}$ \\
SRWB & $12.6 \pm 0.6^{\mathrm{a}}$ & $16.8 \pm 2.1^{\mathrm{b}}$ & $9.3 \pm 0.6^{\mathrm{b}}$ \\
AC Brio & $10.4 \pm 0.4^{\mathrm{cb}}$ & $14.5 \pm 1.6^{\mathrm{cb}}$ & $7.3 \pm 0.1^{\mathrm{c}}$ \\
AC Tahoe & $11.1 \pm 0.5^{\mathrm{b}}$ & $14.4 \pm 2.6^{\mathrm{cb}}$ & $8.4 \pm 0.9^{\mathrm{cb}}$ \\
Snowbird & $9.9 \pm 0.4^{\mathrm{c}}$ & $13.3 \pm 0.6^{\mathrm{c}}$ & $6.9 \pm 0.1^{\mathrm{c}}$ \\
Win A & $8.5 \pm 0.3^{\mathrm{d}}$ & $13.4 \pm 1.6^{\mathrm{c}}$ & $7.5 \pm 0.7^{\mathrm{cb}}$ \\
Win B & $8.7 \pm 0.1^{\mathrm{d}}$ & $12.3 \pm 0.6^{\mathrm{c}}$ & $8.1 \pm 2.2^{\mathrm{cb}}$ \\
\hline
\end{tabular}

${ }^{*}$ Different letters in column for each solvent are significantly different $(P<0.05)$ in Duncan's multiple range and values are means of triplicates \pm standard deviations (SD)

$\mathrm{TPC}=$ Total Phenolic Content as mg Ferulic Acid Equivalent (FAE) $/ \mathrm{g}$

\subsubsection{DPPH}

The free radical scavenging activity of different WB extracts with three different solvent systems was studied and the results are presented in Table 4.11. 
Table 4-11 DPPH (\%) of wheat bran extracts with different solvent systems

\begin{tabular}{llll}
\hline Sample & Acidified ethanol & $50 \%$ acetone & $100 \%$ acetone \\
\hline \hline WB & $43.7 \pm 1.2^{\mathrm{d}}$ & $28.3 \pm 1.2^{\mathrm{a}}$ & $22.3 \pm 3.6^{\mathrm{a}}$ \\
SRWB & $45.5 \pm 2.1^{\mathrm{dc}}$ & $20.8 \pm 1.6^{\mathrm{b}}$ & $17.6 \pm 1.9^{\mathrm{a}}$ \\
AC Brio & $48.3 \pm 0.3^{\mathrm{a}}$ & $17.6 \pm 1.5^{\mathrm{cb}}$ & $21.6 \pm 3.3^{\mathrm{a}}$ \\
AC Tahoe & $48.6 \pm 0.1^{\mathrm{a}}$ & $13.7 \pm 2.2^{\mathrm{cd}}$ & $20.9 \pm 1.7^{\mathrm{a}}$ \\
Snowbird & $47.4 \pm 0.3^{\mathrm{ba}}$ & $20.7 \pm 6.5^{\mathrm{b}}$ & $20.6 \pm 4.7^{\mathrm{a}}$ \\
Win A & $47.0 \pm 1.1^{\mathrm{bac}}$ & $7.0 \pm 1.6^{\mathrm{e}}$ & $20.8 \pm 4.0^{\mathrm{a}}$ \\
Win B & $46.1 \pm 0.7^{\mathrm{bc}}$ & $10.3 \pm 0.8^{\mathrm{ed}}$ & $19.6 \pm 3.4^{\mathrm{a}}$ \\
\hline
\end{tabular}

* Different letters in column for each solvent are significantly different $(P<0.05)$ in Duncan's multiple range and values are means of triplicates \pm standard deviations (SD)

$\mathrm{DPPH}=1$, 1-diphenyl-2-picrylhydrazyl as \%DPPH inhibition after $30 \mathrm{~min}$

Solvents used for antioxidant extractions had significant effects $(P<0.05)$ on DPPH scavenging activity. Regardless of wheat variety, the acidified ethanol extracts exhibited the greatest \%DPPH inhibition values, followed by $100 \%$ acetone and $50 \%$ acetone extracted WB samples. Antioxidant activity ranged from $6.97 \%$ to $48.62 \%$ for all WB extracts. Free radical chain reaction is a chemical mechanism which is similar to lipid oxidation in food products. Also free radical scavengers are a group of potential antioxidants to be used as food additives and nutraceuticals. ${ }^{178}$ By measuring DPPH, it provides an idea about the total free radical scavenging capacity of each WB extract. ${ }^{179}$

In 1999, Mageed and Fadel extracted WB antioxidants with hexane, chloroform, and ethanol, and compared their capacities by suppressing lipid peroxidation in cooked beef kept at $4{ }^{\circ} \mathrm{C}$ for 7 days. Their results showed ethanol extracts to be more effective than other solvents in the inhibition of lipid oxidation during 7 days of storage. ${ }^{180}$ Their results indicate that ethanol may be more effective than less polar solvents for extracting 
lipohilic antioxidants capable of inhibiting the formation of aldehyde compounds generated from lipid peroxidation; compounds which are detectable with a TBA (thiobarbituric acid) test. Further analysis of WB extract composition is needed to explain the different observations and correlations between TPC and radical scavenging activities.

Our finding shows that WB contains significant free radical scavenging activities against DPPH. It is reported that each solvent system extracts different antioxidant components depending on their polarities, and each antioxidant compound is likely to exhibit different free radical scavenging properties depending on the nature and mechanism of the free radicals used for the antioxidant measurements assays. ${ }^{112}$ Therefore, more research is needed to investigate the chemical components involving total antioxidant activity

\subsubsection{ORAC}

ORAC measures antioxidant inhibition of peroxyl radical and represents radical chain-breaking antioxidant activity by $\mathrm{H}$-atom transfer. ${ }^{110}$ Acidified ethanol, 50\% acetone, and $100 \%$ acetone extracts of the same WB samples showed different ORAC values as shown in Table 4.12, suggesting that extraction solvents may affect the overall estimation of ORAC values of a selected WB sample. The greatest ORAC values was observed in the acidified ethanol extracts for all WB samples, followed by $50 \%$ acetone and $100 \%$ acetone extracts. Our findings indicated that acidified ethanol was a suitable solvent for extracting WB antioxidants with high ORAC values and it is parallel with literature findings. ${ }^{181}$ 
It has been reported each solvent extracts different antioxidant components depending on their polarities, and each type of antioxidant compound is likely to exhibit different free radical scavenging properties depending on the nature and mechanism of the free radical used. ${ }^{112}$ Also, it has been emphasized that the extraction solvents need to be optimized for each type of wheat material used. ${ }^{181}$

Table 4-12 ORAC ( $\mu$ mole of TE/g WB) values of different wheat cultivars extracted with different solvent systems

\begin{tabular}{llll}
\hline Sample & Acidified ethanol & $50 \%$ acetone & $100 \%$ acetone \\
\hline HRWB & $104.5 \pm 0.8^{\mathrm{ba}}$ & $97.3 \pm 5.4^{\mathrm{a}}$ & $19.3 \pm 2.0^{\mathrm{b}}$ \\
SRWB & $112.5 \pm 7.4^{\mathrm{a}}$ & $102.3 \pm 4.0^{\mathrm{a}}$ & $23.6 \pm 1.0^{\mathrm{b}}$ \\
AC Brio & $89.4 \pm 2.4^{\mathrm{c}}$ & $52.1 \pm 4.7^{\mathrm{cb}}$ & $14.02 \pm 0.5^{\mathrm{c}}$ \\
AC Tahoe & $91.1 \pm 4.9^{\mathrm{c}}$ & $62.2 \pm 7.8^{\mathrm{b}}$ & $21.4 \pm 0.2^{\mathrm{b}}$ \\
Snowbird & $89.1 \pm 5.9^{\mathrm{c}}$ & $42.53 \pm 7.8^{\mathrm{c}}$ & $12.63 \pm 0.7^{\mathrm{dc}}$ \\
Win A & $70.5 \pm 4.8^{\mathrm{d}}$ & $46.4 \pm 0.5^{\mathrm{c}}$ & $19.3 \pm 1.0^{\mathrm{b}}$ \\
Win B & $101.6 \pm 0.5^{\mathrm{b}}$ & $42.8 \pm 1.5^{\mathrm{c}}$ & $33.1 \pm 6.3^{\mathrm{a}}$ \\
\hline
\end{tabular}

* Different letters in column for each solvent are significant different $(P<0.05)$ by Duncan's multiple range test and values are means of triplicates \pm standard deviations (SD)

ORAC $=$ Oxygen Radical Absorbance Capacity as $\mu$ mole TE (Trolox Equivalent) $/ g$

Two-way ANOVA showed that cultivar, solvent and their interactions were significantly different $(P<0.0001)$ for TPC, DPPH and ORAC values as shown in Table 4.13. Average TPC, DPPH and ORAC values were the highest in winter wheats and lowest in spring wheats. For both DPPH and ORAC results, the two-way analysis showed that acidified ethanol extracts gave the highest results, whereas for TPC analysis, 50\% acetone gave the highest values compared to the other extraction solvents. 
Table 4-13 The two way ANOVA for cultivar and solvent effects on antioxidant activity measurements; TPC, DPPH and ORAC

\begin{tabular}{lccc}
\hline CULTIVAR & TPC $^{\mathrm{a}}$ & DPPH $^{\mathrm{b}}$ & ORAC $^{\mathrm{c}}$ \\
\hline HRWB & $16.90^{\mathrm{a}}$ & $31.47^{\mathrm{a}}$ & $73.72^{\mathrm{a}}$ \\
SRWB & $12.92^{\mathrm{b}}$ & $27.95^{\mathrm{bc}}$ & $79.49^{\mathrm{a}}$ \\
AC Brio & $10.73^{\mathrm{dc}}$ & $29.15^{\mathrm{ba}}$ & $51.85^{\mathrm{c}}$ \\
AC Tahoe & $11.30^{\mathrm{c}}$ & $27.75^{\mathrm{bc}}$ & $58.22^{\mathrm{b}}$ \\
Snowbird & $10.02^{\mathrm{d}}$ & $29.58^{\mathrm{ba}}$ & $48.09^{\mathrm{dc}}$ \\
Win A & $9.80^{\mathrm{d}}$ & $24.94^{\mathrm{d}}$ & $45.40^{\mathrm{d}}$ \\
Win B & $9.70^{\mathrm{d}}$ & $25.33^{\mathrm{dc}}$ & $59.16^{\mathrm{b}}$ \\
& & & \\
SOLVENTS & & & \\
acidified ethanol & $10.13^{\mathrm{b}}$ & $46.67^{\mathrm{a}}$ & $94.12^{\mathrm{a}}$ \\
50\% acetone & $15.86^{\mathrm{a}}$ & $16.90^{\mathrm{c}}$ & $63.67^{\mathrm{b}}$ \\
100\% acetone & $8.89^{\mathrm{c}}$ & $20.50^{\mathrm{b}}$ & $20.47^{\mathrm{c}}$ \\
\hline
\end{tabular}

\footnotetext{
* Different letters in column for each analysis are significantly different $(P<0.0001)$ in Duncan's multiple range tests and values are means of triplicates

${ }^{a}$ TPC $=$ Total Phenolic Content as mg Ferulic Acid Equivalent (FAE) $/ \mathrm{g}$

${ }^{\mathrm{b}} \mathrm{DPPH}=1$, 1-diphenyl-2-picrylhydrazyl as \%DPPH inhibition after $30 \mathrm{~min}$

${ }^{\mathrm{c}} \mathrm{ORAC}=$ Oxygen Radical Absorbance Capacity as $\mu$ mole TE (Trolox Equivalent) $/ \mathrm{g}$
}

\subsection{Conclusion}

The current study investigated and compared total AR contents, and their relative homologue composition in WB samples using traditional and $\mathrm{SC}-\mathrm{CO}_{2}$ extraction methods. The results showed that winter wheats had higher levels of ARs than spring wheats. The highest amount of ARs was found in HRWB for both extraction methods and the most common AR homologues were C21:0, followed by C19:0, C23:0, C17:0 and C25:0. Due to the amphiphilic nature of ARs, the extraction yield was higher for the traditional extraction using a polar organic solvent than for $\mathrm{SC}-\mathrm{CO}_{2}$ extraction. Even 
though a higher AR yield was obtained by traditional solvent extraction, a valuable extract rich in ARs was been obtained by $\mathrm{SC}-\mathrm{CO}_{2}$ from WB. Further studies need to be conducted to determine the optimum extraction conditions of $\mathrm{SC}-\mathrm{CO}_{2}$, such as particle size, soaking before extraction, and temperature. Alkaline hydrolysis and liquid-liquid partitioning steps were carried out to extract ester linked phenolics from both winter wheats and their fractions, including soluble free, soluble conjugated and bound forms. HPLC analysis allowed identification of ten major phenolic acid peaks; FA was detected as the predominant phenolic acid found mostly in bound fractions. Also, the same fractions were analyzed for flavonoid content by HPLC and six major flavonoid peaks were identified. Further studies are needed for the antioxidant activity of each fraction and different cultivars from different regions need to be analyzed to compare soluble free, soluble conjugated and bound phenolic content. For the final part of the study, the effects of extraction solvent on TPC, DPPH and ORAC were investigated. This study reports that different extraction solvents have effects on the antioxidant activity measurements for WB samples. Findings from this study suggest that $50 \%$ acetone might be the recommended solvent for the preparation of WB extracts for TPC measurements and acidified ethanol could be used to extract WB bioactive components for both DPPH and ORAC measurements. 


\subsection{Connecting Statement to Chapter 5}

In Chapter 4, the characterization of wheat bran ARs by HPLC was studied for different wheat cultivars. The extraction process included both traditional and supercritical fluid extraction methods. Also, this chapter gave us comparison of antioxidant measurement of different wheat varieties extracted with three different solvent systems.

In Chapter 5, the potential of WB soluble polysaccharides on the growth of bacterial survival and growth in yogurt as well as the antioxidant activity of the polysaccharides was investigated.

The work presented in Chapter 5 has been used to prepare one conference presentation (poster). Also, one publication will be submitted, as follows.

- Gunenc, A. and Hosseinian, F., 2012. Canadian Institute of Food Science and Technology (CIFST)-50 ${ }^{\text {th }}$ National Conference, Potential of adding red hard wheat bran on yogurt production and antioxidant activity measurements: total phenolics, DPPH, and ORAC, Niagara Falls, ON, Canada.

- Gunenc, A., and Hosseinian, F., 2013. Wheat bran enhances lactic acid bacterial survival and growth in yogurt and hold high antioxidant capacity 


\section{Chapter 5 Wheat bran enhances lactic acid bacterial survival and growth in yogurt and hold high antioxidant capacity}

\subsection{ABSTRACT}

This study investigated the potential of hard red wheat bran (HRWB) polysaccharides to enhance bacterial survival and growth in yogurt as well as the antioxidant activity of the polysaccharides. Initially, the highest amount of bran that could be added to milk was determined, and $4 \%$ bran addition was found to be the optimum concentration of HRWB in yogurt for the entirety of the study. The microbial count, $\mathrm{pH}$ and total titratable acidity (TTA) were measured weekly in yogurt samples supplemented with probiotic bacteria (Lactobacillus acidophilus and Bifidobacterium lactis) in combination with $4 \%$ HRWB for 28 days stored at $4{ }^{\circ} \mathrm{C}$. Results showed that there were significant differences $(P<0.05)$ in total bacterial count $(9.1 \log \mathrm{CFU} / \mathrm{mL})$, $\mathrm{pH}(4.8)$ and TTA (1.4\%) in the presence of 4\% HRWB in yogurts compared to controls at the end of the cold storage period. The water extractable polysaccharides (WEP) from HRWB were enzymatically ( $\alpha$-amylase and protease) treated and dialyzed to obtain pure extract. The WEP exhibited strong antioxidant activity with an ORAC value of 52.48 $\mu \mathrm{mol}$ trolox equivalent/g, DPPH inhibition of $31.64 \%$, and TPC value of $4.22 \mathrm{mg} \mathrm{FAE} / \mathrm{g}$. Also, the total dietary fiber (DF) content of HRWB was determined as $53 \%$ of the bran; $88.6 \%$ of DF as insoluble dietary fiber (IDF) and $11.4 \%$ of DF soluble dietary fiber (SDF). HPLC analysis of the alkaline hydrolyzed DF fractions showed that IDF had the highest phenolic acid and flavonoid content compared to SDF. This study suggests that wheat bran polysaccharides act as a good source of prebiotic, enhancing lactic acid bacteria growth in a yogurt model. 


\subsection{Introduction}

Epidemiological studies have shown that whole grain consumption has been related to a decreased risk of chronic diseases ${ }^{30}$, Particulary obesity ${ }^{182}$, type II diabetes, ${ }^{183}$ cardiovascular disease, ${ }^{184}$ and cancer. ${ }^{185}$ In addition to risk reduction, whole grain consumption has been reported to improve gut health. ${ }^{186}$ Wheat is one of the main wholegrain cereals consumed in the world, containing about $13 \%$ dietary fibre and at least $2 \%$ bioactive compounds apart from fibre. Wheat bran (WB) on the other hand, is made up of $45 \%$ dietary fibre and $7 \%$ bioactive compounds. ${ }^{187} \mathrm{WB}$ can be used in food products due to its high fibre content and antioxidant properties. ${ }^{188}$

Prebiotics may be defined as "a nondigestible food ingredient or soluble dietary fibre". Cereal grain oligosaccharides acts as prebiotics and increase levels of beneficial bacteria in the large bowel, thereby improving gut health. ${ }^{189}$ It is a nutritional substrate for probiotics in the colon that have the potential to improve host health. ${ }^{190}$ Prebiotic oligosaccharides can be manufactured by extraction from plant materials through microbial/enzymatic synthesis and enzymatic hydrolysis of polysaccharides. ${ }^{191}$ In addition to the prebiotic potential of cereal oligosaccharides, polyphenolic compounds such as ferulic, or gallic acids are related to prevention of diseases through possible mechanisms such free radical quenching, chelation of transition metals, reducing peroxides and stimulation of the antioxidant enzyme system. ${ }^{192}$ Potential protective effects of phenolic acids and of oligosaccharides as prebiotics deserve to be studied in more depth. ${ }^{30}$ Probiotics and prebiotics are mostly used in fermented dairy products that are the number one products of functional foods worldwide. ${ }^{193}$ Therefore, research is essential for the discovery of new high-value bio-products and their potential use in 
functional foods or nutraceuticals. The objectives of this study were to; 1) determine acceptable concentration of red hard wheat bran (HRWB) in yogurt production, 2) calculate the prebiotic effects of HRWB addition on microbial counts (CFU), $\mathrm{pH}$ and total titratable acidity (TTA) in yogurt samples containing starter cultures with or without probiotic bacteria, 3) measure antioxidant activity of water-extractable polysaccharides by using oxygen radical absorbance capacity (ORAC) and 2, 2-diphenyl-1-picryhydrazyl radical (DPPH) radical scavenging activity assays as well as total phenolic count (TPC), and 4) determine total dietary fiber content (soluble and insoluble dietary fiber) and analyze the phenolic acid and flavonoid composition of each fiber fraction.

\subsection{Materials and method}

\subsubsection{Materials}

All detailed information about the materials used for this study is included in section 2.3.2.

\subsubsection{Sample preparation}

Hard red wheat bran (HRWB) was provided by Kraft Canada. Samples were grounded to a $0.5 \mathrm{~mm}$ particular size by using a cyclone sample mill (UDY Corporation, CO, USA) from Agriculture-Canada (Ottawa, ON, Canada), freeze dried and stored in sealed plastic bags at $-20^{\circ} \mathrm{C}$ until further use.

\subsubsection{Probiotic activity}

\subsubsection{Milk Preparation and Sample Concentration}

Pasteurized whole milk (homogenized 3.25\%) was purchased at the local market. The milk was heated at $85^{\circ} \mathrm{C}$ for 15 minutes, cooled down to $42^{\circ} \mathrm{C}$ in a water bath and 
transferred into $50 \mathrm{ml}$-sterile test tubes. The starter cultures, probiotics, and different HRWB concentrations were added and incubated at $42^{\circ} \mathrm{C}$ until the yogurt reached approximately $\mathrm{pH} 5.0 .^{194}$

For determining optimum concentration of HRWB in yogurt without disrupting the fermentation, concentrations of $0,2,4,6$, and $8 \%(\mathrm{w} / \mathrm{v})$ bran were added to $50 \mathrm{ml}$ pasteurized milk and incubated at $42{ }^{\circ} \mathrm{C}$ until the completion of fermentation. All yogurt treatments of varying HRWB concentrations were carried out in triplicates.

\subsubsection{Microbial cultures}

For yogurt preparation, the starter cultures, Lactobacillus delbrueckii ssp. bulgaricus and Streptococcus salivarius ssp. thermophilus, were employed. Lactobacillus acidophilus and Bifidobacterium lactis were used as probiotic 1 and 2 respectively. The lyophilized bacteria were rehydrated and stored at $-80^{\circ} \mathrm{C}$ in $32 \%$ glycerol before use. For each strain, $10 \mathrm{~mL}$ sterile aliquots of Man Rogosa Sharpe (MRS) broth liquid media was used to grow microorganisms and incubated at $42^{\circ} \mathrm{C}$ for 24 hours. For preparation of the stock culture, the activated tubes were used after three successive rinses with sterilized distilled water. Then the cultures were diluted with sterilized milk $\left(121^{\circ} \mathrm{C}\right.$ for $15 \mathrm{~min}$ in an autoclave) to obtain a concentration of $6.5 \log$ bacteria cells $/ \mathrm{ml}$. Once the dilutions were made for from the stock culture $0.5 \mathrm{~mL}$ of each yogurt starter culture and $1.0 \mathrm{~mL}$ of required probiotics (pro 1 and 2) were added to the tubes depending on the treatments. The probiotics were added to yogurt samples as shown in Table 5.1 and the initial $\mathrm{pH}$ recorded. There were a total eight yogurt trials; four contained HRWB and four served as controls lacking HRWB. Samples with HRWB were compared against samples lacking HRWB as a probiotic substrate. All tubes were incubated at $42{ }^{\circ} \mathrm{C}$ for fermentation and 
$\mathrm{pH}$ was measured after $4 \mathrm{~h}$ and $1 \mathrm{~h}$ thereafter. When the $\mathrm{pH}$ reached approximately 5 , samples were stored at $4{ }^{\circ} \mathrm{C} .{ }^{194}$

Table 5-1 The experimental design used to evaluate the effect of addition of HRWB sample on probiotic viability in different yogurt trials.

\begin{tabular}{|c|c|}
\hline Yogurt trials $^{*}$ & Sample coding \\
\hline $\mathrm{Y}$ & $\mathrm{Y}$ \\
\hline $\mathrm{Y}+$ Pro 1 & $\mathrm{Y}+1$ \\
\hline$Y+$ Pro 2 & $\mathrm{Y}+2$ \\
\hline $\mathrm{Y}+$ Pro $1+$ Pro 2 & $\mathrm{Y}+1+2$ \\
\hline $\mathrm{Y}+\mathrm{HRWB}$ & YB \\
\hline $\mathrm{Y}+\mathrm{HRWB}+$ Pro 1 & $\mathrm{YB}+1$ \\
\hline $\mathrm{Y}+\mathrm{HRWB}+$ Pro 2 & $\mathrm{YB}+2$ \\
\hline $\mathrm{Y}+$ HRWB + Pro $1+$ Pro 2 & $\mathrm{YB}+1+2$ \\
\hline
\end{tabular}

\subsubsection{Microbial count}

Total microbial counts were carried out on days 1, 7, 14, 21 and 28 in triplicate for each batch at different dilutions; four serial dilutions of 1 to 10 . A $5 \mu$ portion from each dilution was plated on MRS agar dishes in triplicate by using a spread plate technique. Colonies were counted after incubation at $42{ }^{\circ} \mathrm{C}$ for 24 hours. ${ }^{194}$ Counted colonies were converted to $\log$ CFU (Colony Forming Unit) / mL usingthe following formula: 


$$
\log \frac{C F U}{m L}=\frac{1000 \mu \mathrm{L} \times \frac{C F U}{\text { plate }}}{5 \mu \mathrm{L}} \times \text { dilution factor }
$$

\subsubsection{4 pH and total titratable acidity (TTA)}

Post-acidification was read at days 1, 7, 14, 21 and 28, using a Denver Instrument UB-5 pH meter (Denver Instrument, Bohemia, New York, USA). For the same days, TTA was also performed by titrating a mixture of yogurt and sterile water $(1: 9, \mathrm{v} / \mathrm{v})$ with $0.1 \mathrm{~N} \mathrm{NaOH}$ using $0.1 \%$ phenolphthalein as an indicator. ${ }^{195}$ The following equation was used to calculate TTA \%:

TTA $\%=$ Dilution factor $(10) \times V_{\mathrm{NaOH}} \times 0.1 \mathrm{~N} \times 0.009 \times 100 \%$

$\mathrm{V}_{\mathrm{NaOH}}$ : Volume of $\mathrm{NaOH}(\mathrm{mL})$ added to neutralize the acid and generate a colour change.

\subsubsection{Extraction of prebiotics (water extractable polysaccharide, WEP)}

Prebiotic or WEP extraction was carried out in triplicate by following the method of Escarnot et al. ${ }^{196}$ Ground HRWB was extracted with distilled water (1:100, w/v) stirred for $4 \mathrm{~h}$ at $70^{\circ} \mathrm{C}$, cooled, centrifuged at $6000 \mathrm{x}$ g for $20 \mathrm{~min}$ in a Thermo Sorval centrifuge (Legend XT Series, Fisher Scientific, Nepean, ON, Canada) and the supernatant was kept. For the purpose of removing starch and proteins/peptides, the enzymes of $\alpha$ amylase and protease from Bacillus lichenformisis were added to the supernatant solution $(20 \mu \mathrm{L} / 100 \mathrm{~mL})$ and stirred at $37{ }^{\circ} \mathrm{C}$ for $24 \mathrm{~h}$. The supernatant was then heated at $95{ }^{\circ} \mathrm{C}$ for $5 \mathrm{~min}$ to inactivate the enzymes, cooled to room temperature and re-centrifuged at $6000 \mathrm{x}$ g for $20 \mathrm{~min}$. The supernatant solution was dialyzed against double distilled water for $48 \mathrm{~h}$ and replaced with fresh distilled water every $6 \mathrm{~h}$ to separate polysaccharides and other materials with a molecular weight cut-off of $3500 \mathrm{D}$ (Spectra/Por dialysis 
membrane, Rancho Dominguez, CA, USA) (Figure 4.1A). The extract solutions were kept at $-20{ }^{\circ} \mathrm{C}$ until further analysis.

\subsubsection{Antioxidant activity}

TPC, DPPH and ORAC measurement of WEP were performed by following sections 2.3.5, 2.3.6 and 2.3.7, respectively.

\subsubsection{Total dietary fiber (DF): soluble (SDF) and insoluble (IDF) extraction}

Insoluble dietary fibre (IDF) and soluble dietary fiber (SDF) of the bran sample (HRWB) were prepared based on AOAC Official Method 991.43 and the study of Guo \& Beta. ${ }^{176}$ Briefly, 5 g of HRWB was subjected to enzymatic digestions; firstly, heat stable $\alpha$-amylase $(250 \mu \mathrm{L}$, boiling water bath for $30 \mathrm{~min})$, then alcalase protease $(50 \mathrm{mg} / \mathrm{mL}$, $500 \mu \mathrm{L}, \mathrm{pH} 7.5,60{ }^{\circ} \mathrm{C}$ water bath for $\left.30 \mathrm{~min}\right)$ and lastly, amyloglucosidase $(1500 \mu \mathrm{L}, \mathrm{pH}$ $4.5,60{ }^{\circ} \mathrm{C}$ water bath for $30 \mathrm{~min}$ ) to remove starch and protein. After centrifugation at 10 , $000 \mathrm{rpm}$, the residue was washed with hot water, ethanol (95\%), and acetone $(95 \%)$; it was then vacuum filtered and referred to as IDF. The combined supernatants from the washings was precipitated in ethanol $\left(80 \%\right.$, preheated to $60{ }^{\circ} \mathrm{C}, 4$ volumes $)$ overnight and referred to as SDF. Both of the fractions (IDF and SDF) were placed in a fume hood and dried at $35-40{ }^{\circ} \mathrm{C}$ overnight to remove organic solvent.

\subsubsection{Phenolic acid extraction from SDF and IDF}

HRWB (100 mg), IDF(100 mg) and SDF (50 mg) were subjected to alkaline

hydrolysis and liquid-liquid partitioning steps for releasing ester linked phenolics ${ }^{176}$ as described in the section 4.3 .4 (bound fraction). The dried alkaline extracts were re- 
dissolved in $\mathrm{MeOH}$ for the determination of phenolic acid and flavanoid content. Extracts were filtered with a $0.45 \mu \mathrm{m}$ PTFE filter before HPLC analysis.

\subsubsection{Statistical analysis}

The experiment was conducted according to a completely randomized design with triplicates. Analysis of variance (ANOVA) was determined using the GLM procedure of SAS (version 9.2, SAS Institute Inc., Cary, NC) and when significant $(P<0.05)$ mean comparison was performed using Duncan's Multiple Range test.

\subsection{Results and discussion}

\subsubsection{Optimizing HRWB concentration}

The first objective of this study was to investigate an acceptable concentration of HRWB in yogurt. As a preliminary study, five different concentrations of HRWB including $0,2,4,6$, and $8 \%(\mathrm{w} / \mathrm{v})$ were employed to determine the optimum concentration. All employed concentrations resulted in fermented yogurt products with an even texture as shown in Figure 5.1B. Microbial viability in standard yogurt (containing only starter cultures) with different concentrations of HRWB was observed over 28 days of cold storage and $\log \mathrm{CFU} / \mathrm{mL}$ data has been shown in Figure 5.1C. This graph gave detailed information about how different concentrations of HRWB affected starter cultures during the storage period and helped in determining the optimum concentration of HRWB. 
There were significant differences $(P<0.05)$ in all HRWB concentrations $(2-8 \%)$ compared to the control yogurt (having $0 \%$ HRWB). The yogurts that included 4 and $6 \%$ HRWB showed significantly higher CFU values compared to the yogurts containing 2 and $8 \%$ HRWB.. Meanwhile, the yogurts containing 6 and $8 \%$ of HRWB absorbed more liquid compared to other concentrations as shown in Figure 5.1B. Therefore, addition of $4 \%$ HRWB in yogurt as in the work ${ }^{197}$ of Agil, et al. was determined to be the optimum concentration that could be added to milk for all yogurt trials as shown in Table 5.1. 

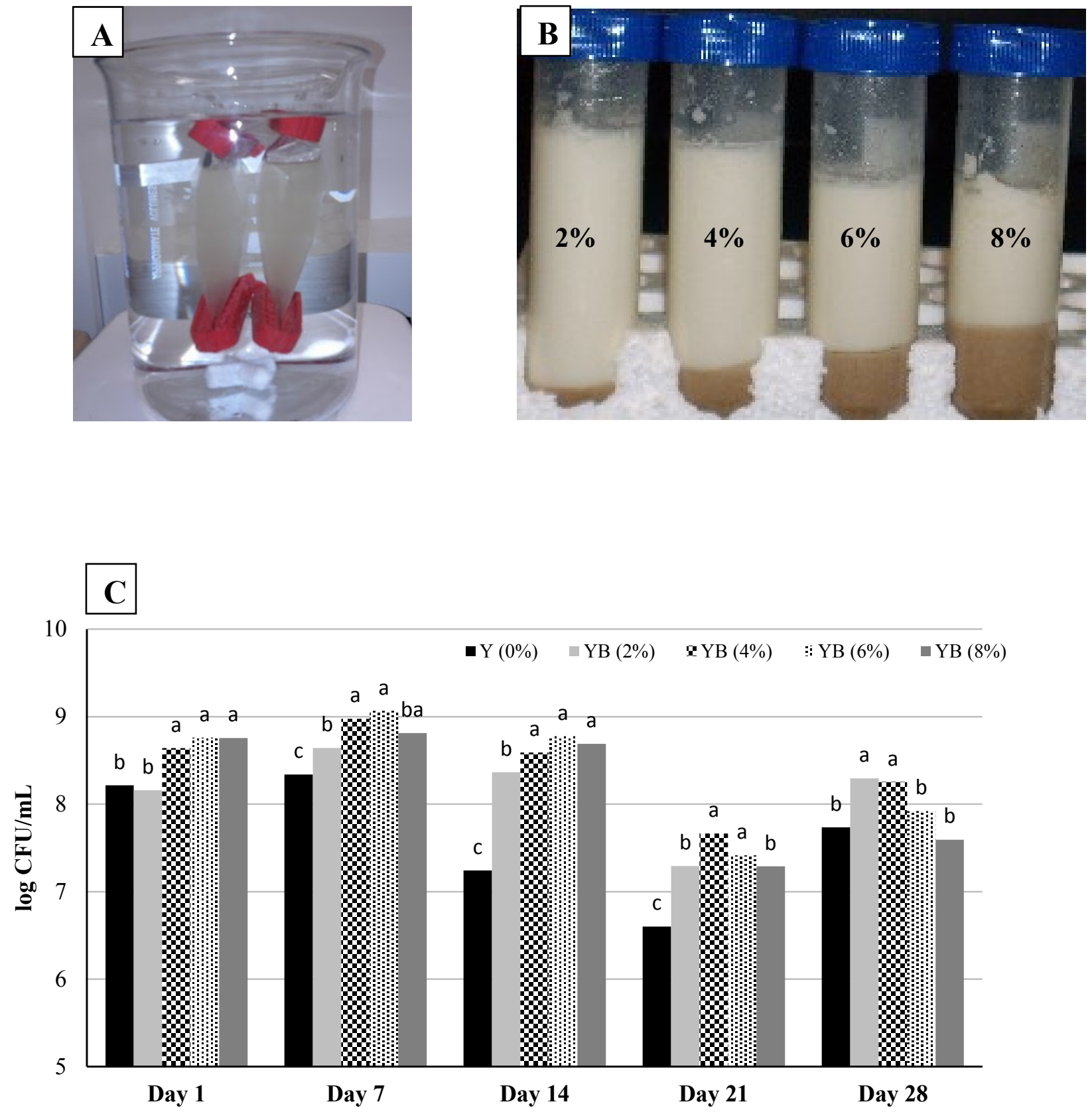

Figure 5-1 A) Dialysis of water extractable polysaccharides, B) Resultant yogurt products with various concentrations of hard red wheat bran (HRWB), C) Total microbial count $(\log \mathrm{CFU} / \mathrm{mL})$ of lactic acid bacteria in yogurt containing starter cultures of Lactobacillus bulgaricus and Streptococcus thermophiles with different concentrations of HRWB including $0,2,4,6$, and $8 \%$ over 28 days cold storage at $4{ }^{\circ} \mathrm{C}$. 


\subsubsection{Prebiotic activity of HRWB addition on microbial count $(\log \mathrm{CFU} / \mathrm{mL})$}

The total microbial counts at days $1,7,14,21$ and 28 in yogurt samples were shown in Figure 5.2. By day 1, microbial counts increased from an initial bacteria count of 6.5 to a range of 8.19-8.31 $\log \mathrm{CFU} / \mathrm{mL}$ in control yogurts, and 8.41-8.65 $\log \mathrm{CFU} / \mathrm{mL}$ in yogurts containing HRWB. There was a significant difference $(P<0.05)$ in the number of bacterial colonies between all yogurt samples containing and lacking HRWB. The yogurt trial, $\mathrm{YB}+1$ (containing HRWB and probiotic 1), had the highest microbial count compared to all other treatments.

By day 7, the number of bacteria in control yogurts ranged from 7.94 to $8.34 \log$ $\mathrm{CFU} / \mathrm{mL}$ whereas the yogurt samples containing HRWB had between 8.87 and $9.15 \log$ $\mathrm{CFU} / \mathrm{mL}$. The bacterial growth increased significantly $(P<0.05)$ in yogurts containing each or both probiotic bacteria in the presence of HRWB compared to their corresponding controls.

By days 14 and 21, the CFU values in yogurt samples with and without HRWB continued to be significantly different yet stable. By the last week of cold storage (day 28), yogurt samples lacking HRWB had markedly lower total bacteria counts of 7.03$7.65 \log \mathrm{CFU} / \mathrm{mL}$ in comparison to $8.31-8.74 \log \mathrm{CFU} / \mathrm{mL}$ viable bacteria found in yogurt samples containing HRWB. It has been suggested that the level of viable bacteria remaining in yogurt after 4 weeks of cold storage should be in the range of 6 to $8 \log$ CFU/mL. ${ }^{198}$ The control yogurts $(\mathrm{Y}, \mathrm{Y}+1, \mathrm{Y}+2, \mathrm{Y}+1+2)$ have remained within range, but the yogurts containing HRWB $(\mathrm{YB}, \mathrm{YB}+1, \mathrm{YB}+2, \mathrm{YB}+1+2)$ had higher bacteria counts in the range of 8.31 and $8.74 \log \mathrm{CFU} / \mathrm{ml}$. Especially the samples containing both pro 1 and 2 had the highest CFU count compared to the other treatment trials on day 28 . 
Overall, the yogurt samples containing HRWB demonstrated significantly higher bacteria counts $(P<0.05)$ throughout the 4 weeks of cold storage in comparison to control samples. These results may be attributed to the high levels of carbohydrates and micronutrient content of wheat and this finding was in agreement with Agil and Hosseinian. ${ }^{199}$ From Figure 5.2, it can be elucidated that in the presence of HRWB, there was an increase in microbial viability in sample treatments consisting of probiotics. It may be a synergistic effect of HRWB on probiotics by comparison to its respective control samples lacking HRWB. These results showed that wheat bran might have a selective effect on increasing probiotic bacteria counts and starter cultures during cold storage from day 0 to day 14 , and to a lesser extent on day 21 . A decreasing or stable trend in $\mathrm{CFU}$ numbers may be a result of nutrient depletion over time. ${ }^{200}$ For all microbes, certain nutrients such as iron and manganese are necessary to promote viability and growth. At the same time, probiotic bacteria have the ability to bind these elements and decrease accessibility by pathogenic bacteria. ${ }^{201}$ Wheat bran consists of high quantities of manganese, and iron, with levels of 4-14 and 2.5-19 g/100g. ${ }^{30}$ So, the presence of wheat bran in yogurt cultures containing probiotics may supply a source of micronutrients and oligosaccharides, selectively stimulating microbial growth or viability of these particular bacterial strains. ${ }^{202}$ 

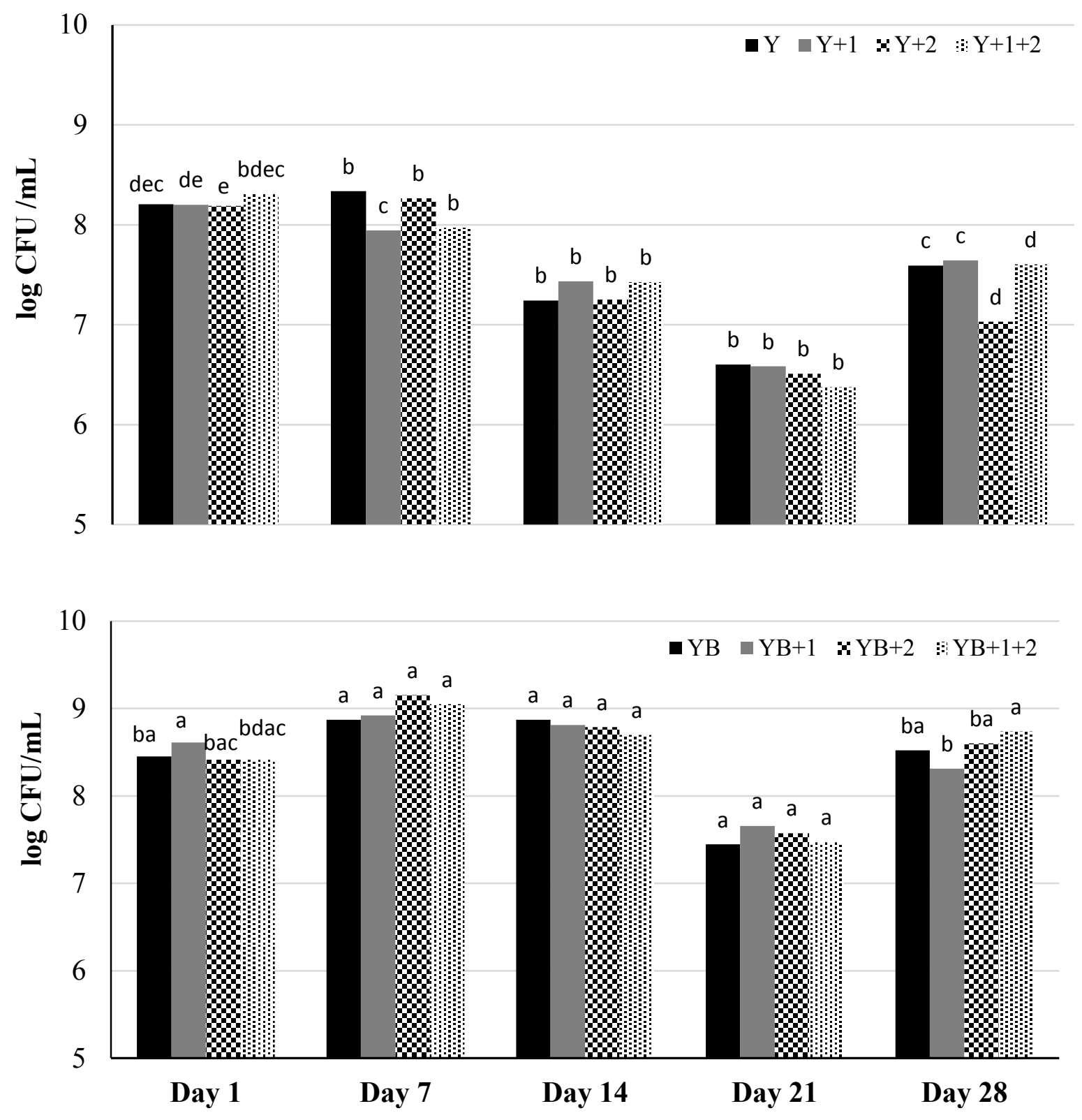

Figure 5-2 Total microbial count $(\log \mathrm{CFU} / \mathrm{mL})$ in control yogurt $(\mathrm{Y}, \mathrm{Y}+1, \mathrm{Y}+2$, $\mathrm{Y}+1+2)$, and yogurt with $4 \%$ wheat bran $(\mathrm{YB}, \mathrm{YB}+1, \mathrm{YB}+2, \mathrm{YB}+1+2)$. Number 1 , and 2 represent probiotic1 and 2. Different letters in columns in the same day are significantly different $(P<0.05)$ in Duncan's multiple range tests 


\subsubsection{Prebiotic activity of HRWB addition on $\mathrm{pH}$ and TTA}

After fermentation, the $\mathrm{pH}$ gradually decreased to 5 in all yogurt samples. After day 1 , yogurts containing HRWB were significantly more acidic $(P<0.05)$ than yogurts lacking HRWB by $0.35-0.87 \mathrm{pH}$ units as shown in Figure 5.3. The $\mathrm{pH}$ of plain yogurt samples decreased steadily in the range of 0.32 to 0.85 units over the 28 day period, whereas the $\mathrm{pH}$ of HRWB yogurts dropped quickly by 0.75 to 0.88 units. The bacteria in yogurt samples with $\mathrm{HRWB}(\mathrm{YB}, \mathrm{YB}+1, \mathrm{YB}+2, \mathrm{YB}+1+2$ samples $)$ were more active and producing more lactic acid, causing the $\mathrm{pH}$ to drop from day 1 to day 28 . With the exception of a significant $0.1 \mathrm{pH}$ unit increase in control yogurts lacking HRWB on day 21, there was a significant difference $(P<0.5)$ between the yogurt treatments lacking versus those containing wheat bran, findings which are parallel to the work of Agil et al. ${ }^{164}$ Also the control yogurts $(\mathrm{Y}+1$ and $\mathrm{Y}+1+2)$ containing probiotic 1 and both probiotics had significantly lower $\mathrm{pH}$ values when compared to control yogurts ( $\mathrm{Y}$ and $\mathrm{Y}+2$ ) containing only starter cultures and probiotic 2 during the 4 week study period. 

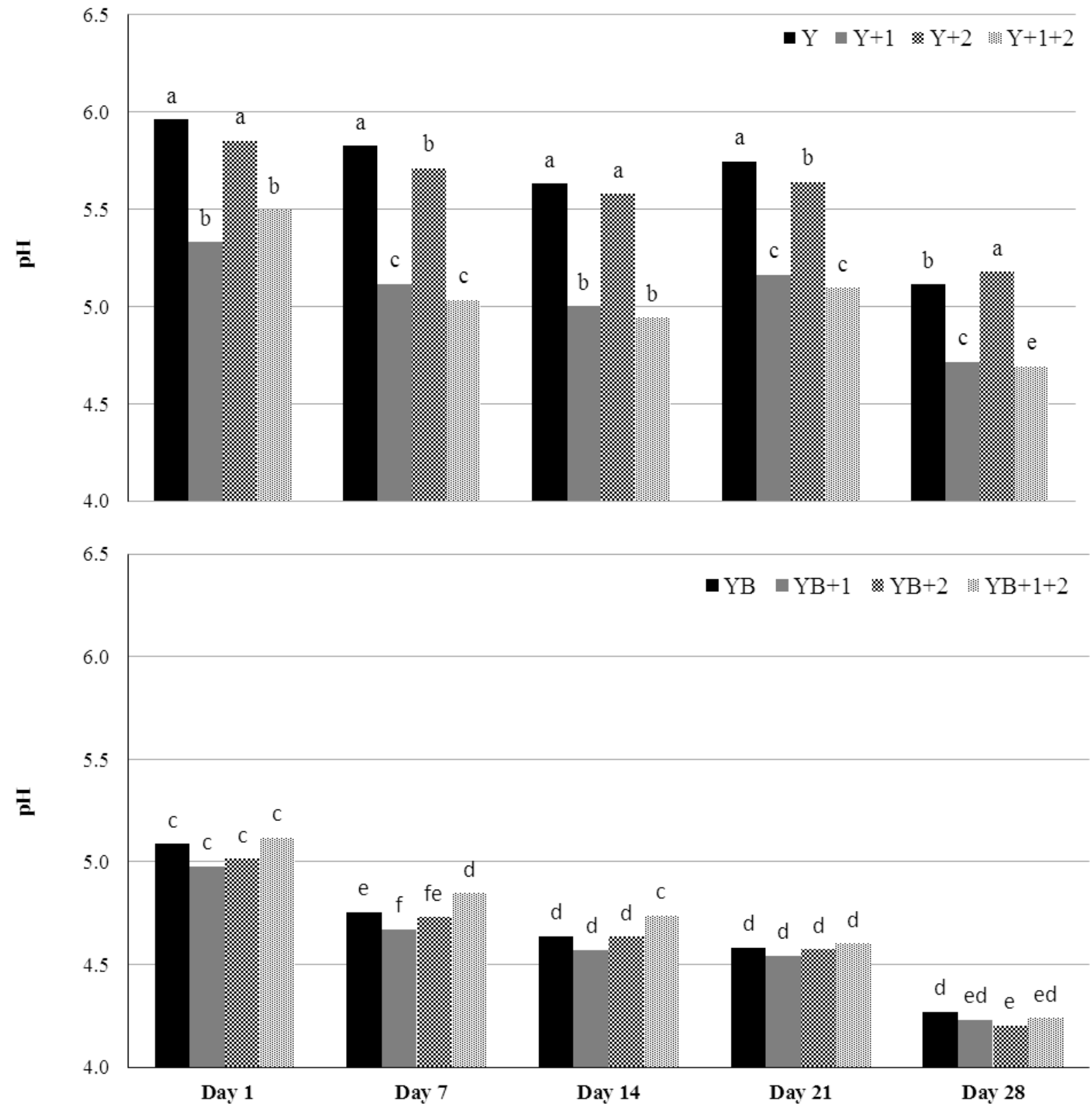

Figure 5-3 The $\mathrm{pH}$ values in control yogurt $(\mathrm{Y}, \mathrm{Y}+1, \mathrm{Y}+2, \mathrm{Y}+1+2)$ and yogurt with 4\% wheat bran $(\mathrm{YB}, \mathrm{YB}+1, \mathrm{YB}+2, \mathrm{YB}+1+2)$. Number 1 and 2 represent probiotic1 and 2 . Different letters in columns in the same day are significantly different $(P<0.05)$ in Duncan's multiple range tests. 
Figure 5.4 illustrates the $\%$ total titratable acidity (TTA) of yogurt samples during the cold storage period. The TTA test was performed to quantify the amount of lactic acid in all yogurt samples. All yogurt samples showed increasing TTA levels as \% lactic acid $(P$ $<0.05)$ as seen in Figure 5.4, indicating that lactic acid is being produced as a result of the growing number of bacteria. The control yogurts had significantly lower TTA levels than corresponding yogurts consisting of HRWB. The reduction in microbial count in all yogurt trials can be attributed to the organic acid accumulation as a result of growth and fermentation. Interestingly, on day 28 , both total microbial count and TTA values for yogurts containing HRWB and both probiotics $(\mathrm{YB}+1+2)$ had the highest values and were significantly different $(P<0.05)$ from their corresponding controls. These results suggest that the addition of wheat bran to yogurt trials caused bacteria to produce more lactic acid, confirming findings obtained from $\mathrm{pH}$ monitoring. 

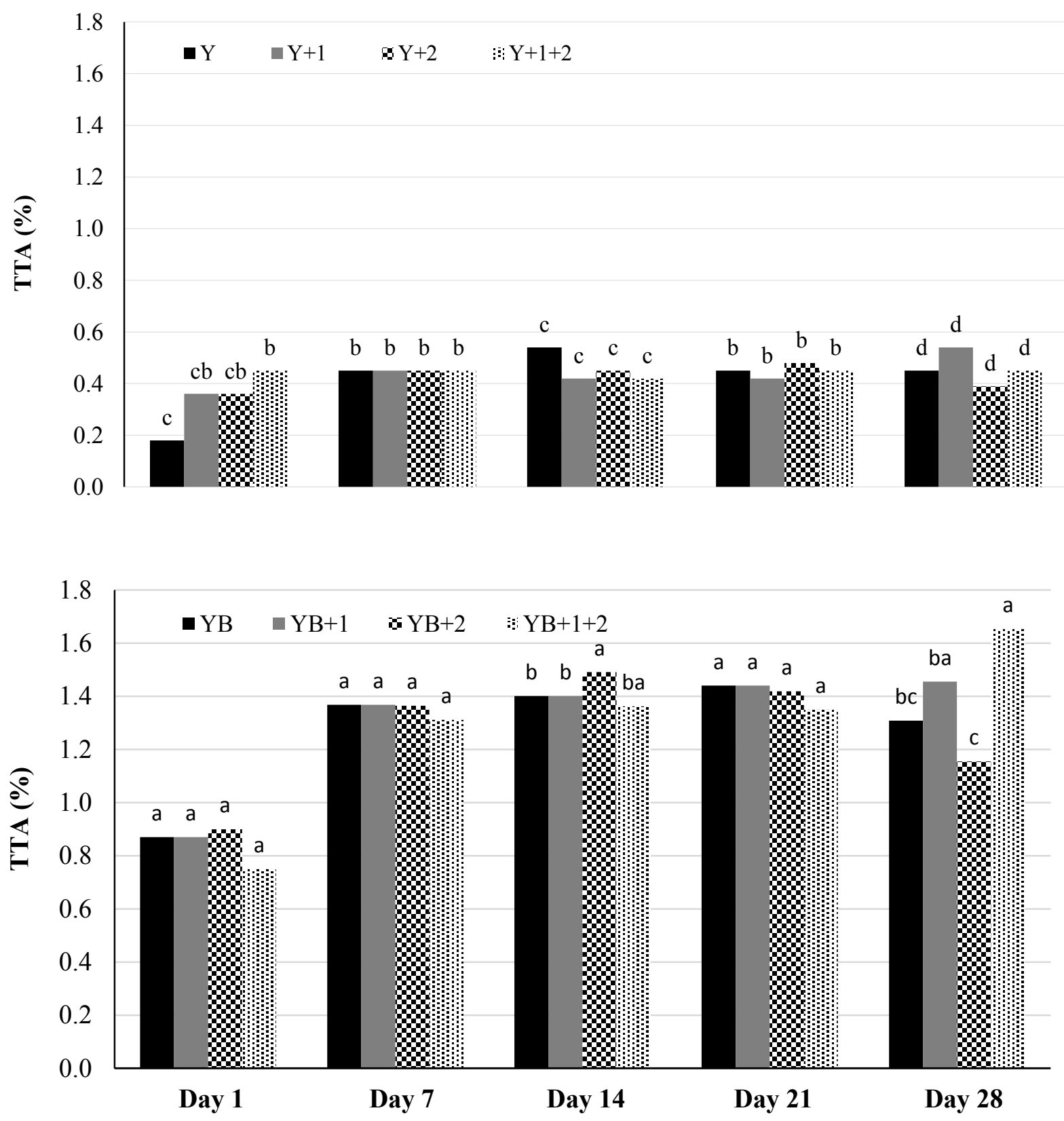

Figure 5-4 \% Total titratable acidity (TTA) values for control yogurts (Y, Y+1, $\mathrm{Y}+2$, $\mathrm{Y}+1+2)$ and yogurt with $4 \%$ wheat bran $(\mathrm{YB}, \mathrm{YB}+1, \mathrm{YB}+2, \mathrm{YB}+1+2)$. Number 1 and 2 represent probiotic1 and 2. Different letters in columns in the same day are significantly different $(P<0.05)$ in Duncan's multiple range tests 


\subsubsection{Total dietary fiber determination}

The total DF content was constituted $53 \%$ of the HRWB. Of this total, $88.6 \%$ was determined to be IDF and $11.4 \%$ SDF. The content and proportion of IDF and SDF are diverse among different types of cereals. This may be due to differences in seed morphology and most cereal grains including wheat, maize and barley have much higher contents of IDF than SDF. Our findings are in agreement with the study of Fardet. ${ }^{30}$

\subsubsection{Phenolic acids and flavonoids content and composition analysis of fractional extraction of phenolics}

In the present study, alkaline hydrolysis and liquid-liquid partitioning steps were carried out to extract ester linked phenolics from IDF, SDF, and HRWB. The separation of phenolic acids in its alkaline extracts was carried out on a RP-HPLC (section 4.3.5.2). The Table 5.2 shows phenolic acid content and composition (mg/g of sample) of IDF, SDF and HRWB.

Table 5-2 Phenolic acid (PA) content and composition (mg/g of sample) of SDF, IDF and HRWB

\begin{tabular}{lllllllllll}
\hline Sample & Total PA* & 1 & 2 & 3 & 4 & 5 & 6 & 7 & 8 & 9 \\
\hline SDF & $0.23 \pm 0.02$ & 0.10 & nd & 0.03 & nd & 0.01 & 0.01 & 0.02 & nd & 0.06 \\
IDF & $1.23 \pm 0.01$ & 0.15 & 0.06 & 0.08 & nd & nd & 0.03 & 0.07 & nd & 0.84 \\
HRWB & $2.64 \pm 0.25$ & 0.24 & nd & 0.17 & 0.89 & 0.04 & 0.05 & nd & 0.11 & 1.13 \\
\hline
\end{tabular}

$* 1=$ gallic, $2=$ proto-catechuic, $3=\mathrm{p}-\mathrm{OH}-$ benzoic, $4=$ chlorogenic, $5=$ vanillic, $6=$ syringic, $7=\mathrm{p}$-coumaric, $8=$ sinapic, $9=$ ferulic, nd $=$ not detected

Values are means of triplicates \pm standard deviations (SD) 

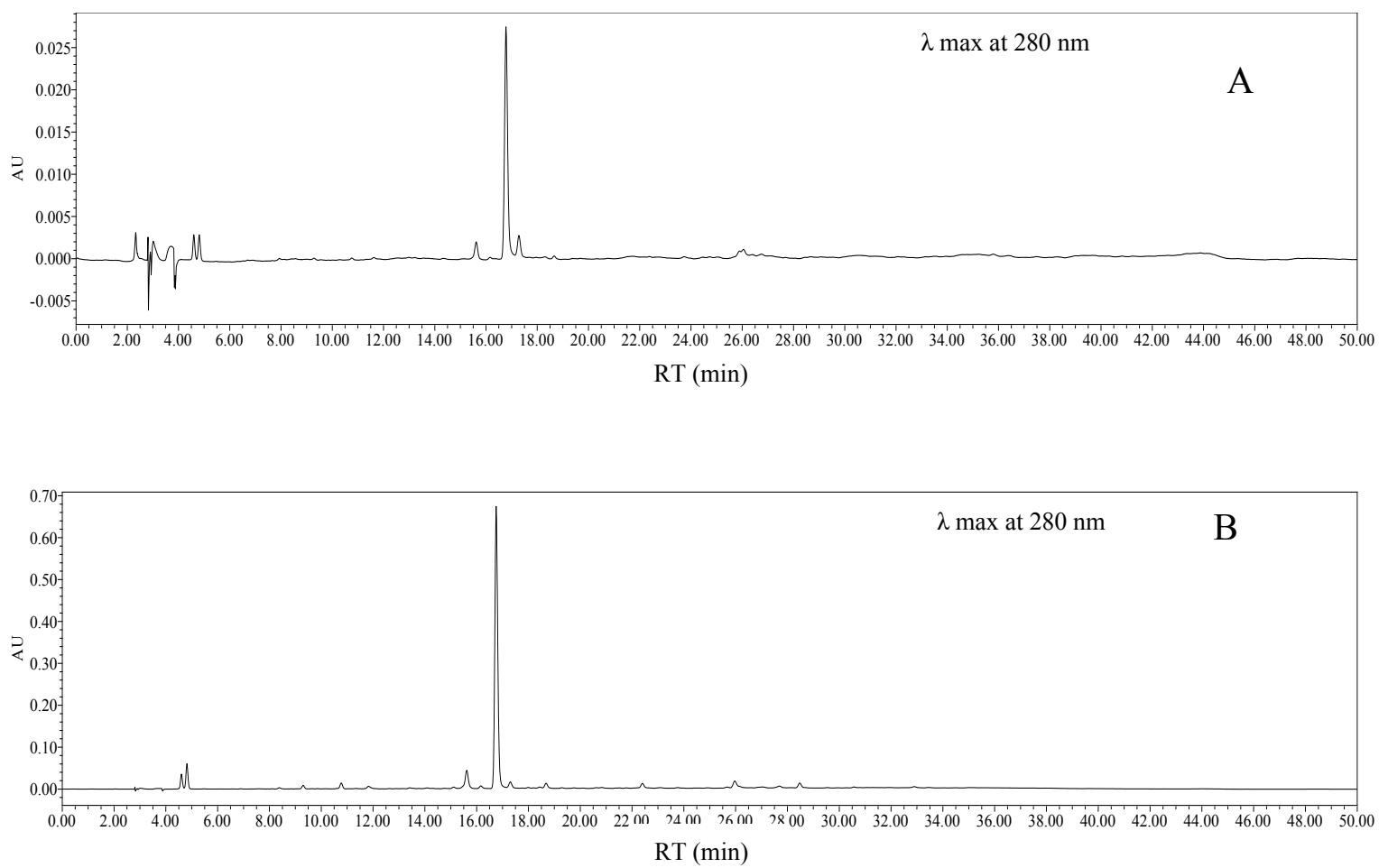

Figure 5-5 HPLC chromatograms of phenolic acid profile of soluble dietary fiber (A), and insoluble dietary fiber (B) of HRWB. Ferulic acid is the most abundant phenolic acid in both fractions. (Table 4.8 descries each standard and corresponding retention times) 
IDF had the higher total phenolic acid content compared to SDF phenolic contents. All three samples, including IDF, SDF and HRWB, had alkaline hydrolysis first, followed by liquid-liquid extraction of bound phenolic acids. When HRWB phenolic acid content was compared to that of DF (IDF+SDF), the HRWB had a greater phenolic acid content of $2.64 \mathrm{mg} / \mathrm{g}$ of sample as opposed to $1.46 \mathrm{mg} / \mathrm{g}$ of sample. This was an expected each step of fractional extraction resulted in some loss of phenolic acids. ${ }^{176}$

With HPLC analysis of the samples, a total of eight phenolic acid peaks were identified in IDF, SDF and HRWB samples (Figure 5.5). They were assigned as gallic, proto-catechmic, p-OH-benzoic, chlorogenic, syringic, p-coumaric, sinapic and ferulic acids. Ferulic acid (FA) was detected as the predominant phenolic acid, and most of FA was found in IDF (40-42\%). The mean FA content ranged from $0.06-1.13 \mathrm{mg} / \mathrm{g}$. High levels of FA in the IDF showed that bound FA was concentrated in those fractions and this finding is in agreement with literature. ${ }^{176}$

Also, the same fractions were analyzed for flavonoid content by HPLC and the results were presented in Table 5.3. With HPLC analysis of the samples, four flavonoid peaks were identified and they respectively assigned as catechin, rutin, quercetin-3-beta glucoside, and epicatechin gallate. The IDF fraction had more flavonoids $(0.22 \mathrm{mg} / \mathrm{g})$ than the SDF fraction $(0.07 \mathrm{mg} / \mathrm{g})$. The phytochemical profiles, especially the flavonoid profiles of wheat, have only been studied in recent years. ${ }^{116}$ Moreover, little is known about the inherent differences in phytochemical profiles. Those biologically active components have not received much attention as the phytochemicals in fruits and vegetables although the increased consumption of whole grains and whole grain products has been linked to a reduced risk of developing chronic diseases. ${ }^{163}$ Our findings of 
flavonoid content were also in close range with Feng et al. ${ }^{177}$ In their study, flavonoids in the bran of four classes of wheat were characterized; three hard red winter classes and one durum class. They reported the mean flavonoid content to be $0.29 \mathrm{mg} / \mathrm{g}$, and our corresponding flavonoid content of total DF and HRWB were 0.29 and $0.35 \mathrm{mg} / \mathrm{g}$ respectively and shown in the Table 5.3.

Table 5-3 Flavonoids content (FC in $\mathrm{mg} / \mathrm{g}$ of sample) of SDF, IDF and HRWB

\begin{tabular}{llllll}
\hline Sample & Total FC* & $1^{*}$ & 2 & 3 & 4 \\
\hline SDF & $0.07 \pm 0.01$ & 0.01 & 0.03 & 0.03 & - \\
IDF & $0.22 \pm 0.09$ & 0.07 & 0.07 & 0.08 & - \\
HRWB & $0.35 \pm 0.12$ & 0.10 & 0.13 & - & 0.12 \\
\hline
\end{tabular}

$* 1=$ catechin, 2 = rutin, $3=$ quercetin-3-beta glucoside, $4=$ epicatechin gallate, nd $=$ not detected Values are means of triplicates \pm standard deviations (SD)

\subsubsection{ORAC}

Table 5.3 illustrates ORAC, DPPH and TPC findings of WEP. The antioxidant activity of WEP was measured using ORAC and DPPH assays. An ORAC assay is used to measure a sample's ability to prevent the oxidation of fluorescein generated by a peroxyl radical (AAPH) and compared with that of known antioxidant, Trolox (a water soluble analogue of vitamin E), at different concentrations ${ }^{161}$ The ORAC value of WEP was found to be $52.48 \mu \mathrm{mol} \mathrm{TE} / \mathrm{g}$ in this study. Within the same experimental conditions, our result showed a higher antioxidant activity than triticale bran, $33.9 \mu \mathrm{mol} \mathrm{TE} / \mathrm{g},{ }^{199}$ and the green and red lentil, 46.1 and $43.1 \mu \mathrm{mol} \mathrm{TE} / \mathrm{g}$, respectively. ${ }^{197}$ In the study of Moore et al.(2005), they have reported the ORAC values of eight Maryland-grown soft wheat 
samples to be in the range of 32.9 to $47.4 \mu$ mole TE/g. ${ }^{149}$ Also in another study, the ORAC of the free fraction of six diverse whole wheats ranged from 19.5 to $37.4 \mu$ mole TE/g. ${ }^{150}$ Our ORAC results are in agreement with both above mentioned studies. This antioxidant activity could be attributed to the presence of bound phenolic acids such as ferulic and p-coumaric acids. ${ }^{203}$ Also, the presence of sugars with acyl groups and/or glycan-polymerization has been reported to have effects on the antioxidant activity of polysaccharides. $^{204}$

Table 5-4 ORAC ${ }^{1}, \mathrm{DPPH}^{2}$ and $\mathrm{TPC}^{3}$ values of $\mathrm{WEP}^{4}$

\begin{tabular}{cc}
\hline Anitoxidant assays & WEP \\
\hline ORAC & $52.48 \pm 2.05$ \\
\%DPPH & $9.32 \pm 0.18$ \\
TPC & $2.96 \pm 0.10$ \\
\hline
\end{tabular}

${ }^{1} \mathrm{ORAC}=$ Oxygen radical absorbance capacity values was calculated as $\mu$ mole Trolox Equivalent (TE)/g of sample. ${ }^{2} \mathrm{DPPH}(\%)=2$, 2-diphenyl-1-picryhydrazyl radical (DPPH) radical scavenging activity assay. ${ }^{3} \mathrm{TPC}=$ Total phenolic count of crude extract was calculated as mg ferulic acid equivalent (FAE)/g of sample.

${ }^{4} \mathrm{WEP}=$ Water extractable polysaccharides and values are means of triplicates \pm standard deviations (SD)

\subsubsection{DPPH assay}

The antioxidant activity of WEP was also measured using DPPH assay. This assay measures the capacity of antioxidants to directly scavenge (react with) DPPH radicals by monitoring its absorbance at $517 \mathrm{~nm}$ and is calculated based on thepercentage discoloration/inhibition of DPPH. ${ }^{114}$ The \%DPPH inhibition of WEP was recorded as $9.32 \pm 0.18 \%$ (Table 5.3), a values similar to to that of Mpofu et al.(2006); they have reported the $\% \mathrm{DPPH}$ inhibition of six wheat cultivars to be in the range of 13.21 to 
$15.06 \%{ }^{132}$

\subsubsection{TPC}

The TPC of HRWB polysaccharide extract was analyzed by Folin-Ciocalteau method and was determined to be $2.96 \pm 0.10 \mathrm{mg} \mathrm{FAE} / \mathrm{g}$ of bran. In the study of Mpofu et al.(2006), they have reported the TPC of six wheat acidified methanol $(\mathrm{HCl} / \mathrm{methanol} /$ water, $1: 80: 10, \mathrm{v} / \mathrm{v})$ extracts to be in the range of 1.70 to $1.99 \mathrm{mg} \mathrm{FAE} / \mathrm{g}$ of wheat. ${ }^{132}$ In wheat, several major phenolic compounds are present including phenolic acids, ARs, flavonoids, phenolic acid diacyl glycerols, phenolic aldehydes and ferulates. ${ }^{30}$ The most abundant phenolic acid compounds belong to the chemical class of hydroxycinnamic acids. The main one is ferulic acid (FA) followed by diferulic acids and by sinapic acid, p-coumaric acid, caffeic acid and benzoic acid derivatives. ${ }^{115}$ About $95 \%$ of phenolic compounds in cereal grains are linked to cell wall polysaccharides. They are covalently bound to polysaccharides through ester bonds and classified as dietary fiber-phenolic compounds. ${ }^{205}$

Consequently, the addition of wheat bran in yogurt might affect the gastrointestinal tract by acting as potential prebiotics improving probiotic viability and functioning as antioxidants, especially after colonic fermentation. ${ }^{30}$ 


\subsection{Conclusions}

The current study investigated the potential of HRWB addition to enhance bacterial survival and growth in yogurt, and the antioxidant activity of WEP by using ORAC and DPPH assays. The outcomes of this study showed that wheat bran enhanced bacterial survival and growth in yogurt over a four week cold storage period. The overall decrease in $\mathrm{pH}$ and increase in TTA in yogurts containing HRWB during the storage period suggest that starter cultures and probiotics could consume wheat bran. Furthermore, WEP showed strong antioxidants activity and this activity is most probably related to its polyphenolic content. This antioxidant activity of WEP confirmed additional beneficiary effects of incorporating these natural bioactive compounds in a yogurt model system. It can be concluded that WB has potential as a source of prebiotics with antioxidant activity for future functional food and neutraceutical applications. Further investigations are needed for the evaluation of probiotic bacterial behavior in the presence of WB by employment of selective media for the enumeration of individual bacterial strains rather than total bacteria counts. Also, it would be interesting to study the sensory and rheological characteristics of yogurt containing wheat bran soluble polysaccharides and useful to know their impacts on yogurt sensorial and textural properties. 


\section{Chapter 6 General Conclusion and Future Directions}

Whole grain consumption has been linked to a lowered risk of chronic diseases such as coronary heart diseases, type II diabetes, and some cancers. These health benefits may be attributed to bioactive components such as phenolics in cereal bran. ${ }^{30}$ This study aimed to extract, characterize, and investigate the bioactivity of ARs in WB. Additionally, incorporation of bioactive phenolics and prebiotics (soluble dietary fiber) were investigated in fermented food products including bread and yogurt.

Many studies have shown that the bioactive compounds produced can be affected by different environmental factors. The effects of genotype, environment and genotypeenvironment interactions may affect the levels of grain antioxidants. The first project of this study aimed to determine the effects of cultivar and region on AR content and homolog composition of $24 \mathrm{WB}$ samples from 4 different regions in Ontario. Moreover, the antioxidant activity of all WB samples was determined by measuring TPC, ORAC and DPPH methods. The cultivar Emmit had the highest amount of total ARs $(1522 \mu \mathrm{g} / \mathrm{g})$ compared to all other cultivars. Although the total AR content was different for each cultivar, the relative saturated (89\%) and unsaturated (11\%) ARs remained in close range. The C21:0 and C19:0 homologues were the most abundant in all WB samples. Only 1\% C15:0 homologue was detected in cultivar Emmit in both Ridgetown and Nairn regions and cultivar Superior in Bath region. Also, the ratios of AR homologues C17:0 /C21:0 has been suggested to be an index for determining the source of a cereal product; ${ }^{137}$ our study showed that these ratios could be used to differentiate between wheat cultivars. The two-way ANOVA analysis has shown that the region, cultivar and the interactions of 
both variables were significant $(P<0.001)$ on all measured parameters. Mean total AR content for different wheat varieties were the highest and lowest in Ridgetown and Bath regions respectively. On the other hand, for each region, cultivars Emmit and AC Morley showed the highest and lowest mean total AR content respectively. Overall, the ANOVA revealed that for each cultivar, the lower the total ARs, the higher the relative \% unsaturated ARs.

A two-way ANOVA showed that region, cultivar and their interactions were significantly different $(P<0.05)$ for TPC, DPPH and ORAC values. Cultivar Emmit grown in Ridgetown had the highest mean TPC content (58 mg FAE/g) and cultivar Harward from Palmerstone region had the lowest mean TPC content (5 mg FAE/g). This wide range shows the dependency of TPC on the type of wheat cultivar analysed. Wheat has major phenolic compounds including phenolic acids, ARs, flavonoids, and phenolic acid diacyl glycerols, phenolic aldehydes and ferulates. ${ }^{30}$ For DPPH results, WB from Ridgetown and Bath regions had the highest and lowest antioxidant activity respectively. The overall \%DPPH values of 41 soft wheats were higher than that of the 40 hard wheats measured. Also, ORAC results revealed that wheat grown in Rigdetown and Bath locations demonstrated the highest and lowest antioxidant capacities respectively. The correlation between total AR content and ORAC results was significantly related $\left(R^{2}=\right.$ $0.80, P<0.05)$. Overall ORAC values of soft wheat $(50 \mu$ mole $\mathrm{TE} / \mathrm{g})$ were higher than hard wheat $(37 \mu$ mole TE/g).

From this first project, it was discovered that the Ridgetown region has a greater potential for selected wheat cultivars to have high AR content. In the same region, the highest content of ARs has been found in cultivar Emmit, and all analyzed WB samples 
had C21:0 as the most common AR homologue. Our results can be used for screening, breeding and genetic modification purposes. For future directions, the same cultivars and some others might be followed up for the following years by recording all data related to environmental conditions (climate, season, degree of maturity, soil conditions, and harvesting periods) to gain a better understanding of the relationships between cultivars rich in ARs and bioactive components. Moreover, thoroughly designed animal and human pilot studies are needed to investigate the bioavailability of wheat ARs from different wheat-based food ingredients and food products.

Cereal products are of the most important and widely consumed foods in the world. Among cereal types, wheat and rye are the most suitable basic ingredients for bread making. ARs are one of the main antioxidative phenolic compounds found in cereals. Thus, the objective of the second project was to investigate the stability of natural levels of ARs in baked breads by analyzing and comparing total and homologue AR levels before and after baking. Moreover, the effects of different solvents (acetone, ethanol, ethyl acetate, and 1-propanol) on the extractability of ARs were investigated. This study also aimed to determine how the baking process affects TPC levels as well as ORAC and DPPH potential.

Seven different bread trials have been prepared for this project, and GC-MS analysis was used to determine total content and homologue composition of ARs in each bread trial. There was a significant difference $(P<0.05)$ in the total AR content extracted by the four different solvents. Acetone gave the highest AR content compared to the other solvents (ethanol, ethyl acetate and 1-propanol). Although the total AR content in each solvent system was different, the relative saturated (92\%) and unsaturated (8\%) ARs 
for WB samples remained in close range and the dominant AR homologues were $\mathrm{C} 21: 0$ and C19:0 as was found in the first project. For the bread samples, a mixture of hot proponal and water was used to extract the ARs as suggested in literature. ${ }^{46}$ This mixture of hot propanol and water was the best solvent recommended to extract ARs with high yield (82.9 $\mathrm{mg} \mathrm{AR} / 100 \mathrm{~g}$ bread D) and release the ARs bound from starch-lipid complexes in bread. All breads tested in this study contained ARs (1.1-82.9 mg/100g bread) and it was shown that AR was did degrade during baking as was expected. Additionally, it was approved that the breads with the highest amount of ARs were also the most elevated and rised of the trial breads, and there was a positive correlation between bread heights and AR content $\left(R^{2}=0.90\right)$.

For TPC analysis, our results indicated that before baking TPC values of both WB samples were significantly different $(P<0.05)$. For ORAC analysis, our results showed that there was significant difference $(P<0.05)$ among sample values as SRWB had higher ORAC values than HRWB. Bread D (containing the highest amount of ARs) had the highest ORAC values in all of the studied bread trials. Overall, the breads high in AR content had higher ORAC values compared to the control bread. Meanwhile, DPPH analysis revealed bread $\mathrm{D}$ to possess the highest antioxidant activity compared to the other bread types. Our study showed that naturally occurring wheat bran ARs were heat stable during baking. ARs with amphiphilic properties, like emulsifiers, can act as dough softeners changing the gelatinization behavior of starch by making complexes with amylose and as a result delay water penetration which has also been suggested to occur during baking. ${ }^{46}$ They may also travel to interfaces between two physical phases and thereby lower surface tension and form dispersions. Further investigations need to be 
done for determining the optimum amount of ARs that should be added in bread or other food model systems.

Traditionally, ARs have been extracted by organic solvents, including acetone, ethanol and ethyl acetate. ${ }^{36,79,164}$ Alternatively, $\mathrm{SC}-\mathrm{CO}_{2}$ can be used for ARs extraction which is non-toxic, non-flammable, inexpensive and faster than traditional methods. ${ }^{66}$ Therefore, the third project aimed to test the potential of using $\mathrm{SC}-\mathrm{CO}_{2}$ for extracting ARs from WB and compare this green/environmentally friendly extraction method with the traditional solvent extraction method. At the same time, this project aimed to evaluate the effects of extraction solvents on the antioxidant potential of WB samples by measuring TPC, ORAC and DPPH values. Lastly, the phenolic profile of WB extract fractions was studied.

Our results showed that there was a significant difference $(P<0.05)$ in the total AR content of all the samples extracted by acetone. Although the total AR was different for each WB sample, the relative saturated and unsaturated ARs for HRWB remained in close range. The AR homologue compositions for all samples were also in close range, with C21:0 and C19:0 being the most. The total AR content of HRWB and SRWB was calculated as 75 and $64 \mathrm{mg} / 100 \mathrm{~g}$ respectively by $\mathrm{SC}-\mathrm{CO}_{2}$ method. It was significantly lower $(P<0.05)$ than the traditional method. When the extraction parameters of both methods were considered, the lower AR content in $\mathrm{SC}-\mathrm{CO}_{2}$ extracts was expected due to solvent polarity differences (acetone versus $\mathrm{SC}-\mathrm{CO}_{2}$ with $10 \%$ ethanol) and extraction times $(24 \mathrm{~h}$ versus $4 \mathrm{~h})$. Although the total AR content differed between extraction methods, the homologue distribution remained analogous. The dominant AR homologues were AR C21:0 and C19:0, results similar to that of the first two projects. Overall, it must 
be emphasized that the highest $\mathrm{AR} /$ extract ratio was obtained when a more polar organic solvents such as acetone was used. In this project, the ratio of ARs/extracts for acetone and $\mathrm{SC}-\mathrm{CO}_{2}$ extraction were recorded to be in the range of $15.6-10.9 \%$ to $6.6-5.1 \%$ for HRWB and SRWB respectively. Moreover, the alkaline hydrolysis and liquid-liquid partitioning steps were carried out to extract bound phenolics from WB. With HPLC analysis, ten major phenolic acid peaks were identified in the fractions which were respectively assigned as gallic, proto-catechmic, p-OH-benzoic, chlorgenic, vanillic, syringic, p-coumaric, sinapic, ferulic and o-coumaric acids. Overall, the total phenolic contents obtained in this study were in the range of 3.70-4.04 mg/g). Also, the same fractions were analyzed for flavonoid content by HPLC and six major peaks identified in the fractions were assigned as pyrogallol, catechin, epicatechin, rutin, quercetin and epicatechin gallate. The phytochemical profiles, especially the flavonoid profiles, have only been studied in recent years. For future directions, the effects of region and cultivar on phytochemical profiles need to be investigated.

Additionally, three solvent systems including acidified ethanol, 50\% acetone and $100 \%$ acetone were used to extract crude $\mathrm{WB}$ antioxidants to investigate the solvent effects on antioxidant activity measurements. Regardless of wheat variety, the $50 \%$ acetone extracts showed the highest TPC values and all winter wheats exhibited significantly higher values $(P<0.05)$ than spring wheats. For DPPH analysis, acidified ethanol gave the highest values compared to the two solvents. Further composition analysis of WB extracts is needed to explain the different observation of the correlations between TPC and DPPH. More research is needed to investigate the chemical components involving total antioxidant activity. Also, acidified ethanol was the solvent 
which gave the highest values for ORAC analysis. Two-way ANOVA revealed that cultivar, solvent, and their interactions were significantly different $(P<0.0001)$ for TPC, DPPH and ORAC values. Our study indicated that mean TPC, DPPH and ORAC values were highest in winter wheats and lowest in spring wheats. For both DPPH and ORAC analysis, acidified ethanol resulted in the highest values, whereas for TPC analysis, 50\% acetone gave the highest values compared to the other extraction solvents used.

Furthermore, whole grain consumption has been reported to improve gut health. ${ }^{186}$ Cereal grain oligosaccharides acts as prebiotics (a nondigestible food ingredients or soluble fibre) and increase the levels of beneficial bacteria in the large bowel thereby improving gut health. ${ }^{189} \mathrm{WB}$ might be used in food products due to its high fibre content and antioxidant properties. ${ }^{188}$ The fourth project aimed to investigate the potential of WB soluble dietary fiber to enhance bacterial survival and growth in yogurt as well as the antioxidant activity of soluble dietary fiber from WB. Our study showed that $4 \%$ WB addition in yogurt was determined to be the optimum concentration of WB for all yogurt trials. This project outcome showed that WB improved the growth of microbial count over a four week cold storage $\left(4^{\circ} \mathrm{C}\right)$ period. The overall decrease in $\mathrm{pH}$ and increase in TTA in yogurts containing HRWB suggest that LAB (lactic acid bacteria) would consume wheat bran during the storage period. It can be concluded that WB has potential as source of prebiotics with antioxidant activity for future functional food and neutraceutical applications. Further in vivo investigations need to be carried out to evaluate the behavior of probiotic bacteria in the presence of wheat bran by employment of selective media for the enumeration of individual bacterial strains rather than total bacteria counts. 


\section{Chapter $7 \quad$ References}

(1) Araus, J. L.; Ferrio, J. P.; Buxo, R.; Voltas, J. The historical perspective of dryland agriculture: lessons learned from 10,000 years of wheat cultivation. Journal of Experimental Botany, 2007, 58, 131-145

(2) Hillman, G. C.; Davies, M. S. Measured domestication rates of wild wheats and barley under primitive cultivation, and their archaeological implications. Journal of World Prehistory, 1990, 4, 157-222.

(3) Gopher, A.; Abbo, S.; Lev-Yadun, S. The 'when', the 'where' and the 'why' of the Neolithic revolution in the Levant. Documenta Praehistorica, 2002, 28, 4962.

(4) Wrigley, C. W. In Wheat: Chemistry and Technology; 4 ed.; Khan, K., Shewry, P. R., Eds.; AACC International Inc.: St.Paul, Minnesota, USA, 2009, p 1-19.

(5) Campbell, A. B.; Shebeski, L. In Wheat in Canada-Past and present; Slinkard, A. E., Fowler, D. B., Eds.; University of Saskatchewan, Division of Extension and Community Relations: Saskatoon, SK, Canada, 1986, p 1-14.

(6) Buller, A. H. R. In Essays on wheat The Macmillan Company, New York, USA., 1919.

(7) DePauw, R. M. In Wheat Science Dynamics: Challenges and opportunities; Chibbar, R. N., Dexter, J. E., Eds.; AACCInternational Inc: St Paul MN, USA, 2011, p 47-58.

(8) AAFC “Outlook for Principal Field Crops: Market Analysis Group/Grains and Oilseeds Division,” 2012. 
(9) Saulnier, L.; Sado, P.-E.; Brandland, G.; Charmet, G.; Guillon, F. Wheat arabinoxylans: exploting variation in amount and composition to develop enhanced varieties. Journal of Cereal Sience, 2007, 46, 261-282.

(10) Surget, A.; Barron, C. Histologie du grain ble. Industrie des Cereales, 2005, 145, 3-7.

(11) Jacobs, D. R.; Gallaher, D. D. Whole grain intake and cardiovascular disease: A review. Current Atherosclerosis Reports, 2004, 6, 415-423.

(12) Osborne, T. B.; Mendel, L. B. The nutritive value of the wheat kernel and its milling products. Journal of Biological Chemistry, 1919, 37:, 557-601.

(13) Girard, A. Diverses parties du grain de proment. Annales De Chimie Et De Physique, 1884, VI, 289.

(14) Nurmi, T.; Lampi, A.-M.; Nyström, L.; Hemery, Y.; Rouau, X.; Piironen, V. Distribution and composition of phytosterols and steryl ferulates in wheat grain and bran fractions. Journal of Cereal Science, 2012, 56, 379-388.

(15) Campbell, G. M. In Handbook of particle breakage; Anonymous, Ed.; Elsevier Science B.V: Oxford, 2007, p 383-419.

(16) Sugden, T. D. In Chemistry and technology; Anonymous, Ed.; Aspen Publishers Inc.: Maryland, 2001, p 140-163.

(17) Evers, T.; Millar, S. Cereal grain structure and development: Some implications for quality. Journal of Cereal Science, 2002, 36, 261-284. 
(18) Peyron, S.; Chaurand, M.; Rouau, X.; Abecassis, J. Relationship between bran mechanical properties and milling behaviour of durum wheat (triticum durum desf.). influence of tissue thickness and cell wall structure. Journal of Cereal Science, 2002, 36, 377-386.

(19) Chick, H. Wheat and bread. A historical introduction. The Proceedings of the Nutrition Society, 1958, 17, 1-7.

(20) Rakszegi, M.; Boros, D.; Kuti, C.; Láng, L.; Bedo, Z.; Shewry, P. R. Composition and end-use quality of 150 wheat lines selected for the HEALTHGRAIN diversity screen. Journal of Agricultural and Food Chemistry, 2008, 56, 9750-9757.

(21) Simmons, L.; Meredith, P. Width, weight, endosperm, and bran of the wheat grain as determinants of flour milling yield in normal and shrivelled wheats. New Zeland Journal of Science, 1979, 22, 1-10.

(22) Crewe, J.; Jones, C. R. The thickness of wheat bran. Cereal Chemistry, 1951, 28 , 40-49.

(23) Shewry, P. R.; Charmet, G.; Branlard, G.; Lafiandra, D.; Gergely, S.; Salgó, A.; Saulnier, L.; Bedo, Z.; Mills, C.; Ward, J. L. Developing new types of wheat with enhanced health benefits. Trends in Food Science Technology, 2012, 25 7077.

(24) Von Braun, J. "The world food situation: New driving forces and required actions," 2007. 
(25) Kumar, P.; Yadava, R. K.; Gollen, B.; Kumar, S.; Verma, R. K.; Yadav, S. Nutritional contents and medicinal properties of wheat: A review. Life Sciences and Medicine Research, 2011, 2011, 1-10.

(26) Belitz, H. D.; Grosch, W.; Schieberle, P. Food Chemistry; 4th ed.; Springere Verlag,: Berlin, Heidelberg, Germany, 2009.

(27) Slavin, J. Why whole grains are protective: biological mechanisms. Proceedings of the Nutrition Society, 2003, 62, 129-134.

(28) Jones, J. M.; Reicks, M.; Adams, J.; Fulcher, G.; Marquart, L. Becoming proactive with the whole-grains messega (Food Science). Nutrition Today, 2004, $39,10-18$.

(29) Zieliński, H.; Kozłowska, H. Antioxidant Activity and Total Phenolics in Selected Cereal Grains and Their Different Morphological Fractions. Journal of Agricultural and Food Chemistry, 2000, 48, 2008-2016.

(30) Fardet, A. New hypotheses for the health-protective mechanisms of whole-grain cereals: what is beyond fibre? Nutrition research reviews, 2010, 23, 65-134.

(31) Frolich, W.; Aman, P.; Tetens, I. Whole grain foods and health-a Scandinavian perspective. Food and Nutrition Research, 2013, 57, 18503-18509.

(32) Saura Calixto, F. Dietary fiber as a carrier of dietary antioxidants: an essential physiological function. Journal of Agricultural and Food Chemistry, 2011, 59, 43-49.

(33) Kozubek, A.; Tyman, J. H. P. Resorcinolic lipids, the natural non-isoprenoid phenolic amphiphiles and their biological activity. Chemical Reviews, 1999, 1, 126. 
(34) Ross, A. B.; Kamal-Eldin, A.; Åman, P. Dietary alkylresorcinols: absorption, bioactivities, and possible use as biomarkers of whole-grain wheat- and rye-rich foods. Nutritional Reviews, 2004, 3, 81-95.

(35) Athukorala, Y.; Hosseinian, F. S.; Mazza, G. Extraction and fractionation of alkylresorcinols from triticale bran by two-step supercritical carbon dioxide. LwtFood Science and Technology, 2010, 43, 660-665.

(36) Mattila, P.; Pihlava, J. M.; Hellstrom, J. Contents of phenolic acids, alkyl- and alkenylresorcinols, and avenanthramides in commercial grain products. Journal of Agricultural and Food Chemistry, 2005, 53, 8290-8295.

(37) Hengtrakul, P.; Lorenz, K.; Mathias, M. Alkylresorcinol homologs in cereal grains. Journal of Food Composition Analysis, 1991, 4, 52-57.

(38) Ross, A. B.; Shepherd, M. J.; Schupphaus, M.; Sinclair, V.; Alfaro, B.; KamalEldin, A.; Åman, P. Alkylresorcinols in cereals and cereal products. Journal of Agricultural and Food Chemistry, 2003, 51, 4111-4118.

(39) Kozubek, A. Determination of octanol/water partition coefficients for long-chain homologs of orcinol from cereal grains. Acta Biochimica Polonica, 1995, 2, 247 252.

(40) Evans LED, W.; Hill, R. D. Variability in the alkylresorcinol content of rye grain. Canadian Journal of Plant Science, 1973, 485-488.

(41) Sedlet, K.; Mathias, M.; Lorenz, K. Growth-depressing effects of 5-npentadecylresorcinol: a model for cereal alkylresorcinols. Cereal Chemistry, $1984,239-241$. 
(42) Al-Ruqaie, I.; Lorenz, K. Alkylresorcinols in extruded cereal brans. Cereal Chemistry, 1992, 69, 472-475.

(43) Baerson, S. R.; Schroder, J.; Cook, D.; Rimando, A. M.; Pan, Z.; Dayan, F. E.; Noonan, B. P.; Duke, S. O. Alkylresorcinol biosynthesis in plants: new insights from an ancient enzyme family? Plant Signaling and Behavior, 2010, 5, 1-4.

(44) Baerson, S. R.; Schroder, J.; Cook, D.; Rimando, A. M.; Pan, Z.; Dayan, F. E.; Noonan, B. P.; Duke, S. O. Alkylresorcinol biosynthesis in plants: new insights from an ancient enzyme family? . Plant Signaling and Behavior, 2010, 10, 12861289

(45) Landberg, R.; Kamal-Eldin, A.; Salmenkallio-Marttila, M.; Rouau, X.; Åman, P. Localization of alkylresorcinols in wheat, rye and barley kernels. Journal of Cereal Science, 2008, 48, 401-406.

(46) Ross, A. B.; Shepherd, M. J.; Schupphaus, M.; Sinclair, V.; Alfaro, B.; KamalEldin, A.; Aman, P. Alkylresorcinols in cereals and cereal products. Journal of Agricultural and Food Chemistry, 2003, 51, 4111-4118.

(47) Kulawinek, M.; Jaromin, A.; Kozubek, A.; Zarnowski, R. Alkylresorcinols in selected Polish rye and wheat cereals and whole-grain cereal products. Journal of Agricultural and Food Chemistry, 2008, 16, 7236-7242.

Andersson, A. A.; Lampi, A. M.; Nystrom, L.; Piironen, V.; Li, L.; Ward, J. L.; Gebruers, K.; Courtin, C. M.; Delcour, J. A.; Boros, D.; Fras, A.; Dynkowska, W.; Rakszegi, M.; Bedo, Z.; Shewry, P. R.; Åman, P. Phytochemical and dietary fiber components in barley varieties in the HEALTGRAIN Diversity Screen. Journal of Agricultural and Food Chemistry, 2008, 21, 9767-9784. 
(49) Chen, Y.; Ross, A. B.; Aman, P.; Kamal-Eldin, A. Alkylresorcinols as markers of whole grain wheat and rye in cereal products. Journal of Agricultural and Food Chemistry, 2004, 52, 8242-8246.

(50) Mullin, W. J.; Emery, J. P. H. Determination of alkylresorcinols in cereal-based foods. Journal of Agricultural and Food Chemistry, 1992, 40, 2127-2130.

(51) Zarnowski, R.; Suzuki, Y. 5-n-Alkylresorcinols from grains of winter barley (Hordeum vulgare L.) Zeitschrift fur Naturforschung C, 2004, 59.

(52) Andersson, A. A.; Kamal-Eldin, A.; Aman, P. Effects of Environment and Variety on Alkylresorcinols in Wheat in the HEALTHGRAIN Diversity Screen (dragger). Journal of Agricultural Food Chemistry, 2010, 58, 9299-9305.

(53) Musehold, J. 5-Alkylresorcinol content influenced through grain size, genetical and environmental conditions. Hodowla Roslin Aklimatyzcja Nasiennictwo, 1975, $19,413-417$.

(54) Ross, A. B.; Chen, Y.; Frank, J.; Swanson, J. E.; Parker, R. S. Cereal alkylresorcinols elevate gamma-tocopherol levels in rats and inhibit gammatocopherol metabolism in vitro. Journal of Nutrition, 2004, 134, 506-510.

(55) Andersson, A. A. M.; Kamal-Eldin, A.; Fras, A.; Boros, D.; Aman, P. Alkylresorcinols in Wheat Varieties in the HEALTGRAIN Diversity Screen. Journal of Agricultural and Food Chemistry, 2008, 21, 9722-9725.

(56) Landberg, R.; Kamal-Eldin, A.; Andersson, R.; Åman, P. Alkyresorcinol content and hmologue composition in durum wheat (Triticum durum) kernels and pasta products. Journal of Agricultural and Food Chemistry, 2006, 8, 3012-3014.

(57) Söderholm, P., the University of Helsinki, 2012. 
(58) Yu, D.; Xu, F. Y.; Zeng, J.; Zhan, J. Type III polyketide synthases in natural product biosynthesis. International Union of Biochemistry and Molecular Biology Life, 2012, 64, 285-295.

(59) Birch, A. J.; Donovan, F. W. Studies in relation to biosynthesis.I.Somepossible routes to derivatives of orcinol and phloroglucinol. Australian Journal of Chemistry, 1953, 6, 360-368.

(60) Funa, N.; Ozawa, H.; Hirata, A.; Horinouchi, S. Phenolic lipid synthasesis by type III polyketide synthases is essential for cyst formation in Azotobacter vinelandii. Proceedings of National Academy of Sciences, 2006, 103, 6356-6361.

(61) Suziki, Y.; kurano, M.; Esumi, Y.; Yamaguchi, I.; Doi, Y. Biosynthesis of 5alkylresorcinol in rice: incorporation of a putative fatty acid unit in the 5alkylresocinols carbon chain. Bioorganic Chemistry, 2003, 31, 437-452.

(62) Verdeal, K.; Lorenz, K. Alkylresorcinols in wheat, rye, and triticale. Cereal Chemistry, 1997, 54, 475-483.

(63) Liukkonen, K.-H.; Katina, K.; Wilhelmsson, A.; Myllymaki, O.; Lampi, A.-M.; Kariluoto, S.; Piironen, V.; Heinonen, S.-M.; Nurmi, T.; Adlercreutz, H.; Peltoketon, A.; Pihlava, J.-M.; Hietaniemi, V.; Poutanen, K. Process-induced changes on bioactive compounds in whole grain rye. Proceedings of Nutrition Society, 2003, 01, 117-122.

(64) Ho, C. H. L.; Cacace, J. E.; Mazza, G. Extraction of lignans, proteins and carbohydrates from faxseeds meal with pressurized low polarity water. Lwt-Food Science and Technology, 2007, 4, 1637-1647. 
(65) Ciftci, O. N.; Ciftci, D.; Jenab, E. In Olive oil-constituents, quality, health properties and bioconversions; Dimitrios, B., Ed.; InTech: 2012.

(66) Francisco, J. d. C.; Danielsson, B.; Kozubek, A.; Dey, E. S. Application of supercritical carbon dioxide for the extraction of alkylresorcinols from rye bran. The Journal of Supercritical Fluids, 2005, 35, 220-226.

(67) McKenzie, L. C.; Thompson, J. E.; Sullivan, R. Green chemical processing in the teaching laboratory: a convenient liquid $\mathrm{CO}_{2}$ extraction of natural products. Green Chemistry, 2004, 6, 355-358.

(68) Landberg, R.; Dey, E. S.; Francisco, J. D. C.; Aman, P.; Kamal-Eldin, A. Comparison of supercritical carbon dioxide and ethyl acetate extraction of alkylresorcinols from wheat and rye. Journal of Composition and Analysis, 2007, $20,534-538$.

(69) Wieringa, G. W. In On the occurance of growth inhibiting substances in rye; Institution of Storage and Processing of Agricultural Produce.: Wageningen, Netherlands, 1967; Vol. Publ.No. 156.

(70) Landberg, R.; Åman, P.; Kamal-Eldin, A. A rapid gas chromatographymass spectrometry method for quantification of alkylresorcinols in human plasma. Analytical Biochemistry, 2009, 385, 7-12.

(71) Nagy, K.; Ross, A. B.; Fay, L. B.; Bourgeois, A.; Kussmann, M. Gas chromatography/tandem mass spectrometry analysis of alkylresorcinols in red blood cells. Rapid Communications in Mass Spectrometry, 2008, 22, 4098-4104. 
(72) Ross, A. B.; Redeuil, K.; Vigo, M.; Rezzi, S.; Nagy, K. Quantification of alkylresorcinols in human plasma by liquid chromatography/tandem mass spectrometry. Rapid Communications in Mass Spectrometry, 2010, 24, 554-560.

(73) Kozubek, A.; Nienartowicz, B. In $X X X$ PTBioch Congress Szczecin, 1994, p 228.

(74) Tluscik, F.; Kozubek, A.; Mejbaum-Katzenellenbogen, W. Alkylresorcinols in rye (Secale cereale L.) grains. VI. Colorimetric micromethod for the determination of alkylresorcinols with the use of diazoniu salt, Fast Blue B. Acta Societatis Botanicorum Ploniae, 1981, 50.

(75) Gajda, A.; Kulawinek, M.; Kozubek, A. An improved colorimetric method for the determination of alkylresorcinols in cereals and whole-grain cereal products. Journal of Food Composition and Analysis, 144, 21, 428-434.

(76) Landberg, R.; Kamal-Eldin, A.; Andersson, A. A.; Aman, P. Analytical procedures for determination of alk(en)ylresorcinols in cereals and cereal products. AACC International, 2009, 4, 25-40.

(77) Sampietro, D. A.; Vattuone, M. A.; Catalan, C. A. N. A new colorimetric method for determination of alkylresorcinols in ground and whole-cereal grains using the diazonium salt Fast Blue RR. Food Chemistry, 2009, 115, 1170-1174.

(78) Ross, A. B.; Aman, P.; Andersson, R.; Kamal-Eldin, A. Chromatographic analysis of alkylresorcinols and their metobolites. Journal of Chromatography A, 2004, 1054, 157-164. 
(79) Zarnowski, R.; Suzuki, Y. Expedient Soxhlet extraction of resorcinolic lipids from wheat grains. Journal of Food Composition and Analysis, 2004, 17, 649663.

(80) Dey, E. S.; Mikhailopulo, K. A food grade approach for the isolation of major alkylresorcinols (ARs) from rye bran applying tailored supercritical carbon dioxide (scCO2) extraction combined with HPLC. The Journal of Supercritical Fluids, 2009, 51, 167-173.

(81) Salek, M.; Brudzynski, A. Composition of alkylresorcinols in grains of various species and varieties of cereals. Acta Aliment. Pol., 1981, 7, 119-126.

(82) Tluscik, F. Localization of the alkylresorcinols in rye and wheat caryopses. Acta Societatis Botanicorum Ploniae, 1978, 47, 211-218.

(83) Kozubek, A. Thin-layer chromatographic mapping of 5-n-alk(en)ylresorcinols homologues from cereal grains. J.Chrom., 1984, 295, 304-307.

(84) Tluscik, F. Determination of cereal 5-n-alkylresorcinol homologues by thin layer chromatography on aluminium oxide. Analytical Chemistry, 1984, 29, 79-84.

(85) Marklund, M.; Landberg, R.; Aman, P.; Kamal-Eldin, A. Comparison of gas chromatography-mass spectrometry and high-performance liquid chromatography with coulometric electrode array detection for determination of alkylresorcinol metabolites in human urine. Journal of Chromatography B, 2011, 879, 647-651.

(86) Kozubek, A.; Geurts Van Kessel, W. S. M.; Demel, R. A. Separation of 5-nalkylresorcinols by reversed-phase high-performance liquid chromatography. Journal of Chromatography A, 1979, 169, 422-425. 
(87) Mullin, W. J.; Wolynetz, M. S.; Emery, J. P. H. A comparison of methods for the extraction and quantification of alk(en)lresorcinols. Journal of Food Composition Analysis, 1992, 5, 216-223.

(88) Seitz, L. M. Identification of 5-(2-oxoalkyl)resorcinols and 5-(2oxoalkenyl)resorcinols in wheat and rye grains. Journal of Agricultural and Food Chemistry, 1992, 40, 1541-1546.

(89) Koskela, A.; Samaletdin, A.; Aubertin-Leheudre, M.; Adlercreutz, H. Quantification of Alkylresorcinols Metabolites in Plasma by High-Performance Liquid Chromatography with Coulometric Electrode Array Detection. Journal of Agricultural and Food Chemistry, 2008, 56, 7678-7681.

(90) Ross, A. B.; Kamal-Eldin, A.; Jung, C.; Shepherd, M. J.; Aman, P. Gas chromatographic analysis of alkylresorcinols in rye (Secale cereale $L$ ) grains. Journal of the Science of Food and Agriculture, 2001, 14, 1405-1411.

(91) Deszcz, L.; Kozubek, A. Higher cardol homologs (5-alkylresorcinols) in rye seedlings. Biochimica et Biophysica Acta, 2000, 1483, 241-250.

(92) Kozubek, A.; Nienartowicz, B. Cereal grain resorcinolic lipids inhibit H2O2induced peroxidation of biological membranes. Acta Biochimica Polonica, 1995, $42,309-315$.

(93) Rejman, J. K. A. Inhibitory effect of natural phenolic lipids upon NADdependent dehryodrogenases and on triglyceride accumulation in 3T3-L1 cells in culture. Journal of Agricultural and Food Chemistry, 2004, 246-250. 
(94) Rejman, J. K. A. Long-chain orcinol homologs from cereal bran are effective inhibitors of glycerophospate dehydrogenase. Cellular and Molecular Biology Letters, 1997, 411-419.

(95) Halliwell, B. Free radicals, proteins and DNA: oxidative damage versus redox regulation. Biochemical Society Transactions, 1996, 24, 1023-1027.

(96) Rice-Evans, C. A.; Diplock, A. T. Current status of antioxidant therapy. Free Radical Biology and Medicine, 1992, 15, 77-97.

(97) Huang, D.; Ou, B.; Prior, R. L. The chemistry behind atnioxidant capacity assays. Journal of Agricultural and Food Chemistry, 2005, 53.

(98) Halliwell, B.; Chirico, S. Lipid peroxidation: its mechanism, measurement, and significance. American Journal of Clinical Nutrition, 1993, 57, 715S-724S.

(99) Smith, C.; Mitchinson, M. J.; Aruoma, O. I.; Halliwell, B. Stimulation of lipid peroxidation and hyrdoxyl-radical generation by the contents of human atherosclerotic lesions. Biochemical Journal, 1992, 286, 901-905.

(100) Wright, J. S.; Johnson, E. R.; DiLabio, G. A. Predicting the Activity of Phenolic Antioxidants: Theoretical Method, Analysis of Substituent Effects, and Application to Major Families of Antioxidants. Journal of the American Chemical Society, 2001, 123, 1173-1183.

(101) Halliwell, B.; Murcia, M. A.; Chiricico, S.; Auroma, O. I. Free radicals and antioxidants in food and in vivo: what they do and how they work. Critical reviews in food science and nutrition, 1995, 35.

(102) Karadag, A.; Ozcelik, B.; Saner, S. Review of Methods to Determine Antioxidant Capacities. Food Analysis Methods, 2009, 2, 41-60. 
(103) Chaudiere, J.; Ferrari-Iliou, R. Intracellular antioxidants:from chemical to biochemical mechnaisms. Food and Chemical Toxicology, 1999, 37, 949-962.

(104) Benzie, I. F. F. Evolution of dietary antioxidants. Comparative Biochemistry and Physiology Part A, 2003, 136, 113-126.

(105) Burton, G. W.; Ingold, K. U. Vitamin E: application of the principles of physical organic chemistry to the exploration of its structure and function. Accounts of Chemical Research, 1986, 19, 194-201.

(106) Hussain, H. H.; Babic, G.; Durst, T.; Wright, J. S. Development of novel antioxidants: design, synthesisi and reactivity. Journal of Organic Chemistry, 2003, 68, 7023-7032.

(107) Winata, A.; Lorenz, K. Antioxidant potential of 5-n-pentadecylresorcinol. Journal of Food Processing and Preservation, 1996, 20, 417-429.

(108) Leopoldini, M.; Russo, N.; Toscano, M. The molecular basis of working mechanism of natural polyphenolic antioxidants. Food Chemistry, 2011, 125, 288-306.

(109) Agil, R.; Hosseinian, F. S. In Bioactive Molecules in Plant Foods; Uruakpa, F. O., Ed.; Nova Science Publishers, Inc.: New York, 2012, p 131-161.

(110) Ou, B.; Woodill-Hampsch, M.; Prior, R. L. Development and validation of an improved oxygen radical absorbance capacity assay using fluorescin as the fluorescent probe. Journal of Agricultural and Food Chemistry, 2001, 49, 46194626. 
(111) Cao, G.; Sofic, E.; Prior, R. Antioxidant and prooxidant behaviour of flavonoids: structure-activity relationships. Free Radical Biology and Medicine, 1997, 22, 749-760.

(112) Moore, J.; Yu, L. L. In Wheat Antioxidants; Yu, L. L., Ed.; John Wiley \& Sons, Inc.: Hoboken, New Jersey, 2008, p 118-172.

(113) Cheng, Z.; Moore, J.; Yu, L. A high-throughput relative DPPH radical scavenging capacity (RDSC) assay. Journal of the Science of Food and Agriculture, 2006, 54, 7429-7436.

(114) Brand-Williams, W.; Cuvelier, M. E.; Berset, C. Use of a free radical method to evaluate antioxidant activity. Lwt-Food Science and Technology, 1995, 28, 2530.

(115) Adom, K. K.; Liu, R. H. Antioxidant activity of grains. Journal of Agricultural and Food Chemistry, 2002, 50, 6182-6187.

(116) Adom, K.; Sorrells, M.; Liu, R. Phytochemical profiles and antioxidant activity of wheat varities. Journal of Agricultural and Food Chemistry, 2003, 51, 78257834.

(117) Buanafina, M. M. d. O. Feruloylation in Grasses: Current and Future Perspectives. M Mol. Plant, 2009, 2, 861-872.

(118) Neyrinck, A. M.; Van Hee, V. F.; Piront, N., De Backer, F.; Toussaint, O.; Cani, P. D.; Delzenne, N. M. Wheat-derived arabinoxylan oligosaccharides with prebiotic effect increase satietogenic gut peptides and reduce metabolic endotoxemia in diet-induced obese mice. Nutriton and Diabetes, 2012, 2, 1-9. 
(119) de vries, R. P.; Visser, J. Aspergillus enzymes involved in degradation of plant cell wall polysaccharides. Microbiology and Molecular Biology Reviews, 2001, $65,497-522$.

(120) Schrezenmeir, J.; de Vrese, M. Probiotics, prebiotics, and synbioticsapproaching a definition. American Journal of Clinical Nutrition, 2001, 73, 361S-364s.

(121) Gill, S. R.; Pop, M.; DeBoy, R. T.; Eckburg, P. B.; Turnbaugh, P. J.; Samuel, B. S.; Gorden, J. I.; Relman, D. A.; Frauser-Liggett, C. M.; Nerlson, K. E. Metagenomic analysis of the human distal microbime. Science, 2006, 312, 13551359.

(122) Saura-Calixto, F.; Serrano, J.; Goñi, I. Intake and bioaccessibility of total polyphenols in a whole diet. Food Chemistry, 2007, 101, 492-501.

(123) Davis, C. D.; Milner, J. A. Gastrointestinal microflora, food components and colon cancer prevention. Journal of Nutritional Biochemistry, 2009, 20, 743-752.

(124) Hevet-Hernandez, D.; Pintado, C.; Rotger, R.; Goñi, I. Stimulatory role of grape pomace polyphenols on Lactobacillus acidophilus growth. International Journal of Food Microbiology, 2009, 136, 119-122.

(125) Shewry, P. R.; Piironen, V.; Lampi, A.; Edelmann, M.; Kariluoto, S.; Nurmi, T.; Fernandez-Orozco, R.; Ravel, C.; Charmet, G.; Andersson, A. A. M.; Aman, P.; Boros, D.; Gebruers, K.; Dornez, E.; Courtin, C. M.; Delcour, J. A.; Rakszegi, M.; Bedo, Z.; Ward, J. L. Effects of region and cultivar on alkylresorcinols content and composition in wheat bran and their antioxidant activity. Journal of Agricultural and Food Chemistry, 2010, 58, 9291-9298. 
(126) DePauw, R. M., Malhi, S.S., Bullock, P.R., Gan, Y.T., McKenzie, R.H., Larney, F.J., Janzen, H.H., Cutforth, H.W., and Wang, H. In The World Wheat Book-A History fo Wheat Breeding; Bonjean, A. P., Angus, W.J., and van Ginkel, M., Ed.; Lavoislier Paris, France, 2011; Vol. 2, p 607-641.

(127) Canada, S. "Seleceted historical date from the census of agriculture," 2009.

(128) Anderson, J. W. Whole grains and coronary heart disease: the whole kernel of truth. American Journal of Clinical Nutrition, 2004, 80, 1459-1460.

(129) Montonen, J.; Knekt, P.; Jarvinen, R.; Aromaa, A.; Reunanen, A. Whole-grain and fiber intake and the incidence of type 2 diabetes. The American Journal of Clinical Nutrition, 2003, 77, 622-629.

(130) Landberg, R.; Kamal-Eldin, A.; Andersson, S.; Johansson, J.; Zhang, J.; Hallmans, G.; Aaman, P. Reproducibility of plasma alkylresorcinols during a 6week rye intervention study in men with prostate cancer. Journal of Nutrition, 2009, 139, 975-980.

(131) Onyeneho, S. N.; Hettiarachchy, N. S. Antioxidant activity of durum wheat bran. Journal of Agricultural and Food Chemistry, 1992, 40, 1496-1500.

(132) Mpofu, A.; Sapirstein, H. D.; Beta, T. Genotype and environmental variation in phenolic content, phenolic acid composition, and antioxidant activity of hard spring wheat. Journal of Agricultural and Food Chemistry, 2006, 54, 1265-1270.

(133) Bushuk, W. Wheat breeding for end-product use. Euphytica, 1998, 100, 137-145.

(134) Ross, A. B.; Kochhar, S. Rapid and sensitive analysis of alkylresorcinols from cereal grains and products using HPLC-Coularray-based electrochemical detection. Journal of Agricultural and Food Chemistry, 2009, 57, 5187-5193. 
(135) Stasiuk, M.; Kozubek, A. Biological activity of phenolic lipids. Cellular and Molecular Life Sciences, 2010, 67, 841-860.

(136) Korycinska, M.; Czelna, K.; Jaromin, A.; Kozubek, A. Antioxidant activity of rye bran alkylresorcinols and extracts from whole-grain cereal products. Food Chemistry, 2009, 116, 1013-1018

(137) Landberg, R.; Andersson, A. A. M.; Aaman, P.; Kamal-Eldin, A. Comparison of $\mathrm{GC}$ and colorimetry for the determination of alkylresorcinol homologues in cereal grains and products. Food Chemistry, 2009, 113, 1363-1369.

(138) Marklund, M.; Landberg, R.; Aaman, P.; Kamal-Eldin, A. Determination of alkylresorcinol metabolites in human urine by gas chromatography-mass spectrometry. Journal of Chromatography, B: Analytical Technologies in the Biomedical and Life Sciences, 2010, 878, 888-894.

(139) Ontario Ministry of tourism, c. a. s. 2009.

(140) Gliwa, J.; Gunenc, A.; Ames, N.; Willmore, W. G.; Hosseinian, F. S. Antioxidant Activity of Alkylresorcinols from Rye Bran and Their Protective Effects on Cell Viability of PC-12 AC Cells. Journal of Agricultural and Food Chemistry, 2011, 59, 11473-11482.

(141) Gao, L.; Wang, S.; Oomah, B. D.; Mazza, G. In Wheat Quality: Antioxidant Activity of Wheat Millstreams; Ng, P., Wrigley, C.W. , Ed.; American Association of Cereal Chemists International: St.Paul, MN, 2002, p 219-233. 
(142) Li, W.; Hydamaka, A.; Lowry, L.; Beta, T. Comparison of antioxidant capacity and phenolic compounds of berries, chokecherry and seabuckthorn. Central European Journal of Biology, 2009, 4, 499-506.

(143) Ou, B.; Huang, D.; Hampsch-Woodill, M.; Flanagan, J. A.; Deemer, E. K. Analysis of antioxidant activities of common vegetables employing oxygen radical absorbance capacity (ORAC) and ferric reducing antioxidant power (FRAP) assays:a comparative study. Journal of Agricultural and Food Chemistry, 2002, 50, 3122-3128.

(144) Hengtrakul, P.; Lorenz, K.; Mathias, M. Alkylresorcinols in U.S. and Canadian wheats and flours. Cereal Chemistry, 1990, 67, 413-417.

(145) Knodler, M.; Kaiser, A.; Carle, R.; Schieber, A. Profiling of Alk(en)ylresorcinols in cereals by HPLC-DAD-APcI-MSn. Analytical and Bioanalytical Chemistry, 2008, 391, 221-228.

(146) Winata, A.; Lorenz, K. Effects of fermentation and baking of whole rye sourdough breads on cereal alkylresorcinols. Cereal Chemistry, 1997, 74, 284287.

(147) Liyana-Pathirana, C. M.; Shahidi, F. Antioxidant properties of commercial soft and hard winter wheats (Triticum aestivum L.) and their milling fractions. Journal of the Science of Food and Agriculture, 2006, 86, 477-485.

(148) Kamal-Eldin, A.; Pouru, A.; Eliasson, C.; Aman, P. Alkyresorcinols as anthioixdants: hydrogen donating and peroxyl radical-scavenging effects. Journal of Food and Agriculture, 2000, 81, 353-356. 
(149) Moore, J.; Hao, Z.; Zhou, K.; Luther, M.; Costa, J.; Yu, L. L. Carotenoid, tocopherol, phenolic acid and antioxidant properties of Maryland-grown soft wheat. Journal of Agricultural and Food Chemistry, 2005, 53, 6649-6657.

(150) Okarter, N.; Liu, C.-S.; Sorrells, M. E.; Liu, R. H. Phytochemical content and antioxidant of six diverse varieties of whole wheat. Food Chemistry, 2010, 119, $249-257$.

(151) Cauvain, S. P.; Cauvain, S. P., Ed.; Woodhead Publishing Limited: Cambridge, England, 2003.

(152) Belitz, H. D.; Grosch, W.; Schieberle, P. In Food Chemistry; Springer-Verlag: Berlin Heidelberg, 2004.

(153) Katina, K. In Bread making, improving quality; Cuvain, S., Ed.; Woodhead publishing limited: Cambridge, 2003, p 485-497.

(154) Li, W.; Pickard, M. D.; Beta, T. Effect of thermal processing on antioxidant properties of purple wheat bran. Food Chemistry, 2007, 104, 1080-1086.

(155) Tokusoglu, O.; Hall, C. In Fruit and cereal bioactives: Sources, chemistry and applications; Taylor \& Francis Group, LLC, CRC: Boca Raton, FL, 2011, p 3-9.

(156) Hirawan, R.; Ser, W. Y.; Arntfield, S. D.; Beta, T. Antioxidant properties of commercial, regular- and whole-wheat spaghetti. Food Chemistry, 2010, 119, $258-264$.

(157) Andersson, U.; Dey, E. S.; Holm, C.; Degerman, E. Rye bran alkylresorcinols suppress adipocyte lipolysis and hormone-sensitive lipase activity. Molecular Nutrition and Food Research, 2011, 55, S290-S293. 
(158) Parikka, K.; Rowland, I. R.; Welch, R. W.; Wahala, L. In vitro antioxidant activity and antiogenotoxicity of 5-n-alkylresorcinols. Journal of Agricultural and Food Chemistry, 2006, 54, 1646-1650.

(159) Esterbauer, H.; Puhl, H.; Dieber-Rotheneder, M.; Waeg, G.; Rabl, H. Effect of antioxidants on oxidative modification of LDL. Annals of Medicine, 1991, 5, 573-581.

(160) Hedkvist, S., Swedish University of Agricultural Sciences, 2009.

(161) Li, W.; Shan, F.; Sun, S.; Corke, H.; Beta, T. Free radical scavenging properties and phenolic content of Chinese black grained wheat. Journal of Agricultural and Food Chemistry, 2005, 53, 8533-8536.

(162) Okarter, N.; Liu, R. H. Health benefits of whole grain phytochemicals. Critical Reviews of Food Science and Nutrition, 2010, 50, 193-208.

(163) Liu, R. H. Whole grain phytochemicals and health. Journal of Cereal Science, 2007, 46, 207-219.

(164) Agil, R.; Oomah, B. D.; Mazza, G.; Hosseinian, F. S. Optimization of alkylresorcinols extraction from triticale bran using response surface methodology. Food and Bioprocess Technology, 2012, 5, 2655-2664.

(165) Bunzel, M.; Ralph, J.; Marita, J. M.; Hatfield, R. D.; Steinhart, H. Diferulates as structural components in soluble and insoluble cereal dietary fibre. Journal of the Science of Food and Agriculture, 2001, 81, 653-660.

(166) Agriculture, U. S. D. o. Database for the flavonoid and proanthocyanidins content of selected foods, 2004 and 2007. (www.nal.usda.gov/fnic/foodcomp/Data), 2007. 
(167) Phenol-explorer In www.phenol-explorer.eu 2010.

(168) Arranz, S.; Silvan, J. M.; Saura-Calixto, F. Nonextractable polyphenols, usually ignored, are the major part of dietary polyphenols: a study on the Spanish diet. Molecular Nutrition and Food Research, 2010, 54, 1646-1658.

(169) Michiels, J. A.; Kevers, C.; Pincemail, J.; Defraigne, J. O.; Dommes, J. Extraction conditions can greatly influence antioxidant capacity assays in plant food matrices. Food Chemistry, 2012, 130, 986-993.

(170) Alothman, M.; Rajeev, B.; Karim, A. A. Antioxidant capacity and phenolic content of selected tropical fruits from Malaysia, extracted with different solvents. Food Chemistry, 2009, 115, 785-788.

(171) Gunenc, A.; Tavakoli, H.; Seetharaman, K.; Mayer, P. M.; Fairbanks, D.; Hosseinian, F. Stability and antioxidant activity of alkylresorcinols in breads enriched with hard and soft wheat brans. Food Research International, 2013, 51, 571-578.

(172) Kim, K.-H.; Tsao, R.; Yang, R.; Cui, S. W. Phenolic acid profiles and antioxidant activities of wheat bran extracts and the effects of hydrolysis conditions. Food Chemistry, 2006, 95, 466-473.

(173) Gunenc, A.; HadiNezhad, M.; Tamburic-Illincic, L.; Mayer, P. M.; Hosseinian, F. Effects of region and cultivar on alkylresorcinols content and composition in wheat bran and their antioxidant activity. Journal of Cereal Science, 2013, 57, 405-410. 
(174) Rebolleda, S.; Beltran, S.; Sanz, M. T.; Gonzalez-Sanjose, M. L.; Solaesa, A. G. Extraction of alkylresorcinols from wheat bran with supercritical CO2. Journal of Food Engineering, 2013, 119, 814-821.

(175) Verma, B.; Hucl, P.; chibbar, R. N. Phenolic acid composition and antioxidant capacity of acid and alkali hydrolysed wheat bran fractions. Food Chemistry, 2009, 116, 947-954.

(176) Guo, W.; Beta, T. Phenolic acid composition and antioxidant potential of insoluble and soluble dietary fibre extracts derived from select whole-grain cereals. Food Research International, 2013, 51, 518-525.

(177) Feng, Y.; Mc Donald, C. E. Comparison of flavonoids in bran of four classes of wheat. Cereal Chemistry, 1989, 66, 516-518.

(178) Chen, X.; Jo, C.; Lee, J. I.; Ahn, D. U. Lipid oxidation, volatiles and color changes of irradiated pork patties as affected by antioxidants. Journal of Food Science, 1999, 64, 16-19.

(179) Yu, L.; Haley, S.; Perret, J.; Harris, M.; Wilson, J. A.; Qian, M. Free radical scavenging properties of wheat extracts. Journal of Agricultural and Food Chemistry, 2002, 50 .

(180) Mageed, M. A. A. E.; Fadel, H. M. F. Evaluation of the antioxidant activity of wheat bran. Indian Journal of Chemical Technology, 1999, 6, 117-120.

(181) Zhou, K.; Yu, L. Effects of extraction solvent on wheat bran antioxidant activity estimation. Lebensmittel-Wissenschaft and Tehcnology, 2004, 37, 717-721. 
(182) van de Vijver, L. P. L.; van den Bosch, L. M. C.; van den Brandt, P. A.; Goldbohm, R. A. Whole-grain consumption, dietary fibre intake and body mass index in the netherlands cohort study. European Journal of Clinical Nutrition, 2009, 63, 31-38.

(183) Murtaugh, M. A.; Jacobs, D. R.; Jacob, B.; Steffen, L. M.; Marquart, L. Epidemiological support for the protection of whole grains against diabetes. Proceedings of the Nutrition Society, 2003, 62, 143-149.

(184) Mellen, P. B.; Walsh, T. F.; Herrington, D. M. Whole grain intake and cardiovascular disease: A meta-analysis. Nutrition, Metabolism and Cardiovascular Diseases, 2008, 18, 283-290.

(185) Schatzkin, A.; Park, Y.; Leitzmann, M. F.; Hollenbeck, A. R.; Cross, A. J. Prospective study of dietary fiber, whole grain foods, and small intestinal cancer. Gastroenterology, 2008, 135, 1163-1167.

(186) Hernandez, L.; Afonso, D.; Rodriguez, E.; Diaz, C. Phenolic compounds in wheat grain cultivars. Plant Foods for Human Nutrition, 2011, 66, 1-8.

(187) Truswell, A. S. Cereal grains and coronary heart disease. European Journal of Clinical Nutrition, 2002, 56, 1-14.

(188) Chen, Z.; Stini, W. A.; Marshall, J. R.; Martínez, M. E.; Guillén-Rodríguez, J. M.; Roe, D. Wheat bran fiber supplementation and bone loss among older people. Nutrition, 2004, 20, 747-751.

(189) Topping, D. Cereal complex carbohydrates and their contribution to human health. Journal of Cereal Science, 2007, 46, 220-229. 
(190) Chakraborti, C. K. The status of synbiotics in colorectal cancer. Life Sciences and Medicine Research, 2011, 20, 1-15.

(191) Grajek, W.; Olejnik, A.; Sip, A. Probiotics, prebiotics and antioxidants as functional foods. Acta Biochimica Polonica, 2005, 52, 665-671.

(192) Aaby, K.; Skrede, G.; Wrolstad, R. E. Phenolic composition and antioxidant activities in flesh and achenes of strawberries (fragaria ananassa). Journal of Agricultural and Food Chemistry, 2005, 53, 4032-4040.

(193) Figueroa-Gonzzalez, I.; Quijano, G.; Ramirez, G.; Cruz-Guerrero, A. Probiotics and prebiotics-perspectives and challenges. Journal of the Science of Food and Agriculture, 2010, 91, 1341-1348.

(194) Santo, A. P.; Silva, R. C.; Soares, F.; Anjos, D.; Gioielli, L. A.; de Oliveira, M. N. Acai pulp addition improves fatty acid profile and probiotic viability in yogurt. International Dairy Journal, 2010, 20, 415-422.

(195) Behrad, S.; Yusof, M. Y.; Goh, K. L.; Baba, A. S. Manipulation of probiotics fermentation of yogurt by cinnamon and licorice: Effects on yogurt formation and inhibition of helicobacter pylori growth in vitro. World Academy of Science, Engineering and Technology, 2009, 60, 590-594.

(196) Escarnot, E.; Aguedo, M.; Agneessens, R.; Wathelet, B.; Paquot, M. Extraction and characterization of water-extractable and water-unextractable arabinoxylans from spelt bran: Study of the hydrolysis conditions for monosaccharides analysis. Journal of Cereal Science, 2011, 53, 45-52. 
(197) Agil, R.; Gaget, A.; Gliwa, J.; Avis, T. J.; Willmore, W. G.; Hosseinian, F. Lentils enhance probiotic growth in yogurt and provide added benefit of antioxidant protection. LWT - Food Science and Technology, 2012, 50, 45-49.

(198) Vasiljevic, T.; Shah, N. P. Probiotics--from metchnikoff to bioactives. International Dairy Journal, 2008, 18, 714-728.

(199) Agil, R.; Hosseinian, F. Dual functionality of triticale as a novel dietary source of prebiotics with antioxidant activity in fermented dairy products. Plant Foods Human Nutrition, 2012, 67, 88-93.

(200) Sengun, I. Y.; Nielsen, D. S.; Karapinar, M.; Jakobsen, M. Identification of lactic acid bacteria isolated from tarhana, a traditional Turkish fermented foods. International Journal of Microbiology, 2009, 135, 105-111.

(201) Bomba, A.; Nemcová, R.; Mudronová, D.; Guba, P. The possibilities of potentiating the efficacy of probiotics. Trends in Food Science and Technology, 2002, 13, 121-126.

(202) Costa, G. E. A.; Queiroz-Monici, K. S.; Machado-Reis, S. M. P.; Oliveira, A. C. Chemical composition, dietary fibre and resistant starch contents of raw and cooked pea, common bean, chickpea and lentil legumes. Food Chemistry, 2006, $94,327-330$.

(203) Hosseinian, F. S.; Mazza, G. Triticale bran and straw: Potential new sources of phenolic acids, proanthocyanidins, and lignans. Functional Foods, 2009, 1, 5764. 
(204) Rao, R. S. P.; Muralikrishna, G. Water soluble feruloyl arabinoxylans from rice and ragi; changes upon malting and their consequence on antioxidant activity. Phytochemistry, 2006, 67, 91-99.

(205) Hatfield, R. D.; Ralph, J.; Grabber, J. H. Cell wall cross-linking by ferulates and diferulates in grasses. Journal of the Science of Food and Agriculture, 1999, 79, 403-407. 